\title{
Nabelschau - \\ Die Forschungleistung österreichischer Ökonomen im nationalen und internationalen Vergleich
}

\author{
Georg Kirchsteiger* und Klaus Ritzberger ${ }^{\dagger}$
}

November 2001

\begin{abstract}
In dieser Arbeit werden alle österreichischen Universitätsinstitute für Volkswirtschaftslehre und Teilgebieten davon, sowie zwei außeruniversitäre Forschungsinstitute, und drei ausländische Institute - als "benchmarks" - hinsichtlich ihrer durchschnittlichen Leistungsfähigkeit in der Forschung verglichen. Es zeigt sich, dass die Institute an den Universitäten Wien und Linz, sowie das Institut für Höhere Studien in Wien eine Spitzenposition im deutschsprachigen Raum einnehmen, aber gegenüber der internationalen Spitze deutlich abfallen.
\end{abstract}

*Institut für Wirtschaftswissenschaften, Universitä t Wien, Hohenstaufengasse 9, A-1010 Wien. email. georg.kirchsteiger@univie.ac.at

${ }^{\dagger}$ Institut für Höhere Studien, Department of Economics and Finance, Stumpergasse 56, A-1060 Wien. email. ritzbe@ihs.ac.at 


\section{Einleitung}

In den letzten Jahren werden in Österreich zunehmend die Strukturen der universitären und außeruniversitären Lehr- und Forschungseinrichtungen disktutiert und reformiert. So wurde das an den Universitäten geltende Dienstrecht verändert, und es ist geplant, die Organisationsform der Universitäten grundsätzlich zu reformieren (Stichwort "Autonomie"). Vermutete oder tatsächlich vorhandene Doppelgleisigkeiten werden in der Öffentlichkeit kritisch angemerkt, und die öffentliche Finanzierung außeruniveritärer Forschungseinrichtungen wird von einflussreichen Politikern in Frage gestellt.

Bei all diesen Diskussionen und Reformen spielt die Qualität und Quantität der Forschung eine geringe Rolle. Dies obwohl Forschung, neben der Lehre, die wichtigste Aufgabe von Universitäten, aber auch von außeruniversitären Forschungseinrichtungen ist. Das mag daran liegen, dass eine Messung der Leistungen in der Forschung als problematisch oder gar unmöglich angesehen wird. Außerhalb Österreichs scheint man jedoch diese Vorbehalte längst fallengelassen zu haben. Nicht nur in den USA, sondern auch in europäischen Ländern, wie England, Spanien oder den Niederlanden sind quantitative Bewertungen wissenschaftlicher Leistungen längst Routine.

Damit wissenschaftliche Leistungen in größerem Ausmaß als bisher auch in Österreich die Diskussionen und Entscheidungen beeinflussen können, ist eine möglichst objektive Bewertung der Leistungen österreichischer Forschungseinrichtungen notwendig. Ziel der vorliegenden Arbeit ist es, für das Fach Volkswirtschaftslehre diese Diskussions- und Entscheidungsgrundlage zu schaffen. Dazu werden hier die Leistungen aller volkswirtschaftlichen (universitären und außeruniversitären) Forschungseinrichtungen in Österreich bewertet und miteinander verglichen.

Vorbild für diese Arbeit ist das holländische System. In den Niederlanden wird seit 1980 jährlich auf der Basis von veröffentlichten Artikeln in internationalen Fachjournalen ein Ranking holländischer ökonomischer Institute und ein Ranking der 40 besten holländischen Ökonomen erstellt. Diese Rankings werden in den Medien veröffentlicht (bis 1991 im Journal "Economische Statistische Berichten", seither im Magazin "Intermediair"), und nicht nur von Insidern, sondern auch von einer interessierten Öffentlichkeit wahrgenommen. Sie haben großen Einfluss auf das Prestige, aber auch auf die Verfügbarkeit von Ressourcen der einzelnen Forschungseinrichtungen. Darüberhinaus haben diese Rankings auch direkte Auswirkungen auf die einzelnen Wissenschafter. Berufschancen und Prestige 
verbessern sich schlagartig, wenn man in der Liste der besten Vierzig aufscheint.

Das Beispiel der Niederlande zeigt auch, dass durch die Anreizwirkungen, die von solchen Rankings ausgehen, Qualität und Quantität der Forschungsleistungen eines Landes erheblich gesteigert werden können. So kommt eine kürzlich erstellte Studie (Kalaitzidakis et.al. (2001)) zu dem Schluss, dass in der zweiten Hälfte der neunziger Jahre die Universität Tilburg auf dem Gebiet der Ökonomie die besten Forschungsleistungen aller europäischen Universitäten vorzuweisen hat. Außerdem, "...it is apparent that countries like ... Netherlands have improved a lot during the last decade and are now producing world class research in economics" (Kalaitzidakis et.al. (2001), S.10). Solche Vergleiche von Forschungsleistungen können also ein wichtiges wissenschaftpolitisches Instrument sein, um die Forschungsleistungen im allgemeinen zu verbessern.

International gibt es bereits einige Studien, die den wissenschaftlichen Output deutschsprachiger (Bommer und Ursprung (1998)) und europäischer (Kalaitzidakis et.al. (1999) und (2001)) Ökonomieinstitute miteinander vergleichen, in denen natürlich auch österreichische Institute inkludiert sind. Andere Studien wiederum betrachten weltweit alle Institute, die im Gebiet der angewandten Ökonometrie veröffentlicht haben (z.B. Baltagi (1999)). Aus diesen Studien ergeben sich einige Hypothesen, deren Überprüfung Anliegen der vorliegenden Arbeit ist. Insbesondere legen die bereits verfügbaren Studien folgende drei Vermutungen nahe.

- Der deutschsprachige Raum bleibt hinter der internationalen Spitze zurück.

- Die besten österreichischen Institute liegen im Spitzenfeld des deutschsprachigen Raumes.

- Zwischen den österreichischen Spitzeninstituten und dem Rest scheint ein erheblicher Abstand zu liegen.

Die erste und zweite Hypothese gehen relativ klar aus den zitierten Studien hervor (siehe auch den jüngsten Bericht an die European Economic Association: Bauwens, Kirman, Lubrano und Protopopescu (2000)). So produziert die London School of Economics (LSE) etwa den doppelten bis dreifachen Forschungsoutput wie die besten deutschsprachigen Universitäten, unter denen sich systematisch österreichische Institute finden. Die besten österreichische Institute wiederum produzieren ein Vielfaches des Outputs der anderen österreichischen Universitäten, soferne diese überhaupt aufscheinen. Das Nichtaufscheinen einiger österreichischer Universitätsinstitute ist ein Indiz für die dritte Vermutung, weil in den Studien nur die Besten aus der jeweiligen Stichprobe angegeben werden. 
Zur Illustration dieser drei Vermutungen gibt die folgende Tabelle 1 einen Überblick über die Produktivitätsmaße, wie sie sich für die hier untersuchten Institute aus anderen Studien ergeben. ${ }^{1}$

\section{(Tabelle 1 etwa hier einfügen.)}

Das weltweite Ranking für das Spezialgebiet der angewandten Ökonometrie (Baltagi (1999)) führt nur drei Institutionen aus der hier verwendeten Stichprobe an, nämlich das Institut für Höhere Studien (IHS) auf Platz 66, die London School of Economics (LSE) auf Platz 106, und die Universität Mannheim auf Platz 247.

Zwei der drei in Tabelle 1 angegeben Studien berücksichtigen jedoch keine außeruniversitäre Einrichtungen, wie das IHS oder das Wirtschaftsforschungsinstitut (Wifo). Und keine gibt einen quantitativen Einblick in die Leistungsfähigkeit der restlichen österreichischen Universitäten in der Forschung. Außerdem erscheint die Art und Weise, wie in diesen Studien die Veröffentlichungen den einzelnen Forschungseinrichtungen zugeordnet werden, in mehrfacher Hinsicht problematisch.

Erstens werden die der Bewertung zu Grunde liegenden Artikel nach den "affiliations", wie sie zum Zeitpunkt der Publikation gegeben sind, den einzelnen Forschungseinrichtungen zugeordnet. Das hat zur Folge, dass unter Umständen ein Artikel einer Institution zugeordnet wird, dessen Autor schon lange nicht mehr an der betreffenden Institution tätig ist. Derartig erstellte Rankings spiegeln also die Forschungsleistungen früherer Mitarbeiter der betreffenden Forschungseinrichtung wider, nicht jedoch notwendigerweise die aktuelle Leistungsfähigkeit der an dem betreffenden Institut tätigen Forscher.

Zweitens werden in diesen Studien zur Zuordnung nur die als "affiliation" angegebene Universität, nicht jedoch das Institut oder der Fachbereich verwendet. Das hat zur Folge, dass z.B. Artikel, die von Mitgliedern eines Mathematikinstituts verfasst wurden, bei Publikation in den entsprechenden wirtschaftswissenschaftlichen Journalen ebenfalls berücksichtigt werden.

Drittens ignoriert die Mehrheit dieser Studien die unterschiedliche Größe der untersuchten Institute, indem sie zwar eine Maßzahl für den Gesamtoutput angeben,

\footnotetext{
${ }^{1}$ In den Studien von Kalaitzidakis et.al., (1999) und (2001), ist das Produktivitätsmaß ("Prod." in der Tabelle) die Anzahl der zu "American Economic Review"-Seiten äquivalenten Seiten, die von der jeweiligen Institution in den 10 bzw. 30 Spitzenjournalen publiziert wurden. Bei Bommer und Ursprung (1998) ist das Produktivitätsmaß die mit Qualitätsindizes von 1 bis 5 gewichtete pro Lehrstuhl publizierte Seitenzahl (in fast zweihundert Journalen). Die Abkürzung "n.a." bedeutet "nicht angegeben".
} 
diesen aber nicht in eine pro-Kopf Größe umrechnen. Da aber die österreichischen volkswirtschaftlichen Institute sehr unterschiedliche Größen aufweisen, führt dies zwangsläufig zu Verzerrungen, die Vergleiche nur sehr bedingt sinnvoll erscheinen lassen.

Viertens werden etwa in Kalaitzidakis et.al. ((1999) bzw. (2001)) nur Artikel, die in den (nach Meinung der Autoren) besten 10 bzw. 30 Journalen erschienen sind, berücksichtigt. Autoren, die auf Spezialgebieten veröffentlichen oder die Probleme von nur regionalem Interesse untersuchen, fallen damit durch den Rost. Umgekehrt benutzt die Studie für den deutschsprachigen Raum (Bommer und Ursprung (1998)) zwar wesentlich mehr Zeitschriften, gewichtet die Publikationen aber nur mit Gewichten zwischen 1 und 5 (entsprechend der Rangordnung in Laband und Piette (1994)), obwohl genauere Maßzahlen für die Bedeutung wissenschaftlicher Fachzeitschriften verfügbar sind.

Um die genannten Probleme zu vermeiden, wird in der vorliegenden Studie

- eine Gesamterhebung der Leistungen aller zur Zeit an volkswirtschaftlichen Instituten tätigen Forscher,

- ein dreistufiges Verfahren und

- eine größere Stichprobe von Zeitschriften

verwendet. Zuerst wurde für alle österreichischen volkswirtschaftlichen Forschungseinrichtungen (zuzüglich der LSE, der Universität Bonn und der Universität Mannheim als "benchmarks") erhoben, welche Wissenschafter zur Zeit bei dieser Forschungseinrichtung beschäftigt sind. (Für Details siehe den nächsten Abschnitt.) Dann wurden die Forschungsleistungen der einzelnen Wissenschafter erhoben, und ihren jeweiligen Instituten zugeordnet. Schließlich wurde die Maßzahl für den wissenschaftlichen Erfolg mit der Anzahl der an der betreffenden Institution Angestellten (mit Forschungsaufgaben) normiert. Damit wird jede Institution nach der durchschnittlichen Leistungen der gegenwärtig an dieser Institution Beschäftigten bewertet. Verzerrungen durch Leistungen früherer Mitarbeiter oder fachnaher, nicht institutszugehöriger Wissenschafter, sowie Größenverzerrungen werden vermieden. Um ein vollständiges Bild zu erhalten, wird in dem vorliegenden Artikel außerdem eine sehr viel größere Anzahl von Journalen, nämlich 243, berücksichtigt. 


\section{Daten und Methoden}

Die Methode der vorliegenden Studie folgt dem holländischen System, mit der Einschränkung, dass hier eine Größenkorrektur verwendet wird. Ausgangspunkt ist die Erhebung aller Angestellten mit Aufgaben in der akademischen Forschung an österreichischen volkswirtschaftlichen Universitätsinstituten, dem Wifo, dem Department of Economics and Finance am IHS, dem Department of Economics an der LSE, am wirtschaftswissenschaftlichen Fachbereich der Universität Bonn und an der Fakultät für Volkswirtschaftslehre der Universität Mannheim. (Eine genaue Liste der berücksichtigten Institute findet sich im Appendix.) Einige Universitäten besitzen neben einem Institut für Volkswirtschaftslehre auch anderere Institute, die sich mit Teilbereichen der Ökonomie (z.B. der Finanzwissenschaft) beschäftigen. Diese Institute wurden ebenfalls in die Studie aufgenommen und dem VWL-Institut der jeweiligen Universität zugeschlagen. Fachnahe, jedoch nicht genuin volkswirtschaftliche Institute (z.B. für Betriebswirtschaftslehre, Ökonometrie oder Operations Research) wurden jedoch ausgeklammert.

Der Nachteil dieser Methode ist, dass damit zum Teil Forscher, die erfolgreich in ökonomischen Journalen veröffentlicht haben, unberücksichtigt blieben, weil sie z.B. an Operations Research oder Betriebswirtschaftsinstituten tätig sind. Der Vorteil ist eine direkte Vergleichbarkeit der Ergebnisse nach der fachlichen Orientierung.

Die Aufnahme der LSE (nach Kalaitzidakis et.al. (1999) die beste europäische Forschungseinrichtung, nach Kalaitzidakis et.al. (2001) die Nummer 20 der Welt), der Universität Bonn (nach Kalaitzidakis et.al. (1999) das beste deutsche Institut, nach Kalaitzidakis et.al. (2001) die Nummer 67 der Welt) und der Universität Mannheim in die vorliegende Studie erfolgte als "benchmark"-Institute, um einen internationalen Vergleich zu ermöglichen.

Die Erhebung der Forscher wurde mit Hilfe der Homepages der betrachteten Forschungseinrichtungen durchgefürt. Dazu wurden die Namen der Wissenschafter, die im November 2000 auf der Homepage des jeweiligen Instituts angeführt waren, aufgelistet. Durch diese Zurechnung ergibt sich also ein gegenwartsorientiertes Bild der Leistungsfähigkeit. So wurde z.B. Robert Kunst (einer der Top-10 in Österreich), der den Grossteil der neunziger Jahre am IHS tätig war, der Universität Wien zugerechnet, an der er seit Ende der neunziger Jahre einen Lehrstuhl innehat. $^{2}$

\footnotetext{
${ }^{2}$ Der als Honorarprofessor an der Universität Linz tätige Karl Aiginger wurde jedoch dem Wifo zugerechnet.
} 
Da jedoch von Doktoranden keine Publikationen in wissenschaftlichen Fachjournalen erwartet werden können, wurden nur Promovierte aufgenommen. Auch für die Größenkorrektur wurde nur die Anzahl der Promovierten verwendet. In den wenigen Fällen, in denen eine Karenzierung aus der Homepage ersichtlich war, wurde der Karenzierte, nicht jedoch die Karenzvertretung in die Studie aufgenommen. Diese Entscheidung wurde getroffen, da im Normalfall der Karenzierte, und nicht die Karenzvertretung, längerfristig an dem betreffenden Institut tätig sein wird. Konsulenten wurden ihrer Stamminstitution zugerechnet, und nicht jener, an der sie als Konsulenten tätig waren.

Als nächster Schritt wurden von jedem der aufgelisteten Forscher die Artikel erhoben, die sie oder er allein oder mit Ko-Autoren zwischen Jänner 1990 und Dezember 1999 in Fachjournalen publiziert hat. Diese Erhebung wurde mittels der Datenbank "Econlit" durchgeführt, welche die 400 wichtigsten ökonomischen Fachzeitschriften enthält und von der American Economic Association geführt wird. Anschließend wurde jeder publizierte Artikel mit dem "Social Science Impact Factors (SSCI)" für das jeweilige Journal im Jahre 1997 gewichtet.

Dieser Faktor, der jedes Jahr von "Institute for Scientific Information" in Philadelphia ermittelt wird, misst für jedes Journal $j$ im Jahre $t$ die Anzahl der Zitationen in der SSCI-Datenbank im Jahre $t$, die sich auf Artikel im Journal $j$ aus den Jahren $t-1$ und $t-2$ beziehen, dividiert durch die Anzahl der in den Jahren $t-1$ und $t-2$ im Journal $j$ publizierten Artikel. Kurz, der SSCI-ImpactFaktor misst die Anzahl der Zitationen der in den letzten zwei Jahren erschienenen Artikel, korrigiert um den durchschnittlichen Umfang der Zeitschrift. Verglichen mit anderen Maßzahlen der Qualität wissenschaftlicher Journale (z.B. den Zahlen in Laband und Piette (1994)) hat der SSCI-Impact-Faktor den Vorteil, dass er den gegenwärtigen und nicht den vergangenen Einfluss misst, den ein Journal auf die ökonomische Wissenschaft hat.

Ein offensichtlicher Nachteil des SSCI-Impact-Faktors ist, dass die Zitationen selbst nicht mit der Bedeutung des zitierenden Journals gewichtet werden. Angesichts der Anzahl der bewerteten Zeitschriften scheitert aber eine solche Korrektur an Berechnungsproblemen. Der große Umfang der SSCI Stichprobe war aber für die vorliegende Studie gerade von Vorteil, da hier nicht nur die besten Journale betrachtet werden sollten, sondern eine möglichst große Auswahl.

Der Umfang der zur Verfügung stehenden Bewertungen war auch entscheidend für die Wahl des Jahres 1997. Dieses Jahr liegt nicht nur etwa in der Mitte der zweiten Hälfte des Beobachtungszeitraumes, sondern in diesem Jahr umfasst die Liste auch 243 Journale, also um fast hundert mehr als im Jahr darauf. Da 
außerdem die Erfahrung zeigt, dass die Ergebnisse kaum sensitiv gegenüber dem Bewertungsjahr sind, überwog der Vorzug der Größe der Stichprobe im Vergleich zum Nachteil der geringeren Aktualität.

Die mit Impact-Faktoren gewichteten Publikationen wurden dann noch um die Anzahl der Ko-Autoren und die Länge des Artikels korrigiert. Erstere Korrektur wurde analog zum holländischen System durchgeführt, zweitere mit einer gröberen Approximation. Um für die Anzahl der Ko-Autoren zu korrigieren, wurde durch die Anzahl der Autoren plus Eins dividiert und mit 2 multipliziert. (Ein Artikel eines einzelnen Autors wurde also mit 1 gewichtet.) Um für die Länge des Artikels zu korrigieren, wurde mit 1 multipliziert, wenn der Artikel mindestens zehn Seiten umfasste, und mit 1/2, wenn er weniger als zehn Seiten lang war. Der Wert eines publizierten Artikels ergab sich also aus der Formel

$$
w=\frac{2 f s}{1+n}
$$

wobei $w$ der Wert des Artikels ist, $f$ der SSCI-Impact-Faktor (im Jahre 1997) des Journals, in dem er veröffentlicht wurde, $s=1$ falls der Artikel mindestens zehn Seiten umfasste und $s=1 / 2$ falls er weniger als zehn Seiten hatte, und $n$ die Anzahl der Autoren.

Die derart ermittelten Werte der einzelnen Publikationen wurden nun für jeden Autor über den Zeitraum Jänner 1990 bis Dezember 1999 summiert. Die resultierende Zahl ist der Impact des einzelnen Autors. Um Institutionen zu bewerten, wurde für jede Institution der Durchschnitt der Impacts der angestellten Forscher ermittelt. Dabei wurden Forscher, die im Beobachtungszeitraum gar nicht publiziert hatten, mit dem Wert Null verbucht. ${ }^{3}$

Die in der vorliegenden Studie ausgewiesenen Maßzahlen für die Forschungsproduktivität sind als gegenwartsorientriertes Maß für die durchschnittliche Leistungsfähigkeit der jeweiligen Institutionen in der Forschung zu interpretieren. Dieser Interpretation liegt natürlich die Annahme zugrunde, dass jemand, der in der Vergangenheit erfolgreich publiziert hat, es auch in der Zukunft tun wird.

\section{Resultate}

Die Resultate der vorliegenden Erhebung bestätigen die "stylized facts", die aus internationalen Studien ersichtlich sind.

\footnotetext{
${ }^{3}$ Die dieser Studie zugrundeliegenden Daten sind auf der Homepage des Bundesministeriums für Wissenschaft, Bildung und Kultur öffentlich zugänglich.
} 
Im einzelnen gibt Tabelle 2 die Produktivität und Platzierung der Institute in der gegenständlichen Stichprobe an. Der "Impact" ist dabei, wie oben ausgeführt, der von den am Ende des Beobachtungszeitraums an der jeweiligen Institution angestellten Forschern durchschnittlich im Zeitraum 1990-99 durch Publikationen in referierten Journalen erreichte (korrigierte) SSCI-Impact-Faktor (siehe (2.1)).

(Tabelle 2 etwa hier einfügen.)

In Übereinstimmung mit den Resultaten von Kalaitzidakis et.al. ((1999), (2001)) ergibt sich, dass die LSE allen deutschsprachigen Forschungseinrichtungen weit überlegen ist. Das Ranking der deutschsprachigen Institutionen wird, wie schon bei Kalaitzidakis et.al. ((1999) und (2001)), vom Institut für Wirtschaftswissenschaften der Universität Wien angeführt. Allerdings produzierte auch dieses Institut nur etwas mehr als ein Drittel des pro-Kopf Outputs der LSE. Die Spitzengruppe des deutschsprachigen Raumes - soweit in der vorliegenden Studie erfasst - liegt relativ knapp beisammen. Universität Wien, IHS, Universität Bonn, Universität Mannheim und die Universität Linz haben in den neunziger Jahren alle etwa einen durchschnittlichen Impact pro Kopf von 1 erreicht. Die restlichen österreichischen Universitätsinstitute und das Wifo kommen nur auf höchstens ein Drittel der Forschungsleistungen der Spitzengruppe. Die Wirtschaftsuniversität Wien, die Universität Klagenfurt und die Universität Salzburg erreichen nicht einmal einen pro-Kopf Impact Faktor von 0.1.

Die Zahlen in Tabelle 2 sind allerdings unter zwei Einschränkungen zu interpretieren. Die erste betrifft die Technische Universität Wien. Diese scheint in vielen internationalen Studien als eine der führenden Institutionen im deutschsprachigen Raum auf. Das liegt daran, dass diese Studien alle Publikationen summarisch der jeweiligen Universität zurechnen. In der vorliegenden Studie wurde jedoch das erfolgreiche Institut für Ökonometrie, Operations Research und Systemtheorie ausgeklammert, und nur das Institut für Volkswirtschaftslehre und Wirtschaftsinformatik berücksichtigt.

Die zweite Einschränkung betrifft das Wifo. Anders als am IHS gibt es am Wifo keine personelle Abgrenzung der Zuständigkeit für akademische Forschung einerseits bzw. für die Auftragsforschung andererseits. In den Nenner des "Impacts" gehen aber alle am Wifo angestellten Forscher ein. Das führt zu einer Unterschätzung der durchschnittlichen Leistungsfähigkeit des Wifo. Geht man etwa 
davon aus, dass Wifo-Mitarbeiter nur ein Drittel ${ }^{4}$ jener Arbeitszeit auf publikationsfähige Forschung verwenden können, den die Mitglieder anderer Institutionen dafür zur Verfügung haben (verdreifacht man also den "Impact"), dann setzt sich das Wifo deutlicher von der Universität Graz ab, bleibt aber auf Platz 7.

Unter diesen Einschränkungen werden dennoch die eingangs erwähnten "stylized facts" eindrucksvoll bestätigt: Der deutschsprachige Raum hinkt hinter der internationalen Spitze her. Österreich kann aber drei Institute im Spitzenfeld des deutschsprachigen Raums platzieren, und verfügt damit zumindest über drei international akzeptable Forschungseinrichtungen im Bereich der Volkswirtschaftslehre. Die restlichen österreichischen Universitätsinstitute und das Wifo weisen jedoch eine sehr viel geringere Forschungsleistung auf. Als Erklärung dieses Befundes lassen sich mehrere Vermutungen ausmachen.

Zunächst scheint im deutschsprachigen Raum die "kritische Masse" zu fehlen. Die führende LSE hat 48 Forscher aufzubieten, während die Universität Wien nur auf 28 kommt, die letztgereihte Universität Salzburg gar nur auf 8. Da moderne Forschung von der Interaktion lebt, ist die geringe Größe deutschsprachiger Institute eine erhebliche Hypothek.

Zweitens schleppen deutschsprachige Universitäten offenbar viel "Ballast" mit. Betrachtet man nämlich die Durchschnittsbildung genauer, so ist es vielfach nicht das Fehlen von besonders erfolgreichen Individuen, das den Durchschnitt drückt. Vielmehr sind es die vielen Unproduktiven, die im Nenner des Durchschnitts eingehen. Bei den vier führenden Instituten in Tabelle 2 liegt der Anteil der Unproduktiven zwischen einem Viertel und einem Drittel. Die mittlere Gruppe weist entsprechende Anteile von 40 bis 50 Prozent auf. Bei den letztgereihten Instituten liegt der Anteil der Unproduktiven (zum Teil erheblich) über 60 Prozent. Offenbar sind deutschsprachige Universitäten kaum in der Lage, Qualitätsansprüche als wirksames Karrierekriterium zu etablieren. Hinzu kommt, dass für pragmatisierte Wissenschafter (ordentliche und außerordentliche Professoren) fast jeder äußere Anreiz fehlt, weiter aktiv Forschung zu betreiben. Gehaltssteigerungen in Abhängigkeit von der Forschungsleistung sind hierzulande unbekannt, und die Vergabe sonstiger Mittel hängt innerhalb österreichischer Universitäten oftmals auch eher von der inneruniversitären Machtverteilung und den Beziehungen zu Ministerien und sonstigen außeruniversitären Institutionen denn von der Forschungsleistung ab. Dass dies mit internationaler Konkurrenzfähigkeit unverträglich ist, liegt auf der Hand.

\footnotetext{
${ }^{4}$ Das entspricht ungefähr dem Anteil der in der akademischen Forschung Beschäftigten am Department of Economic and Finance am IHS.
} 
Drittens wirkt sich die Dominanz der Lehre, insbesondere der Anfängerausbildung, im System der Massenuniversität deutscher Prägung negativ auf den Erfolg der Forschung aus. Während eine Elite-Ausbildung der Forschung förderlich zu sein scheint (wie die Beispiele LSE und IHS zeigen), ist die Massenausbildung eher hinderlich. ${ }^{5}$

Viertens entspricht die Ressourcenausstattung hierzulande nicht den internationalen Standards. Äußerst beschränkte Budgets für Forschungsaufenthalte und Tagungsteilnahmen, wie sie in Österreich verordnet werden, sind international unüblich. Damit wird der internationale Kontakt der Forscher behindert, und sie produzieren am "Markt" vorbei. Dazu kommt ein unflexibles Gehaltsschema, das es letzlich verbietet, internationale Spitzenkräfte adäquat zu bezahlen.

Fünftens läßt sich eine ausgeprägte "Insider"-Mentalität an deutschsprachigen Universitäten beobachten. Dies führt oftmals zu Kollusion, und nicht selten zur Fortsetzung eines einmal eingeschlagenen Weges der niedrigen Qualität. Nicht nur die geringe Frequenz der "Blutauffrischung" an österreichischen Universitäten wirkt negativ auf die Forschung, auch Neuberufungen sind deshalb keine Garantie für Qualitätsverbesserung.

Dass sich einige österreichische Institute dennoch als konkurrenzfähig erweisen, ist wahrscheinlich ein historischer Glücksfall. Durch die Präsenz des IHS in Wien, das ein qualitativ hochwertiges und umfangreiches Gastprofessorenprogramm betreibt, blieb die international Einbindung österreichischer Forscher weitgehend erhalten. Außerdem leidet der Standort Wien durch die Präsenz zweier Spitzeninstitute nicht so sehr an Größennachteilen. Und schließlich haben es die Spitzeninstitute offenbar verstanden, den Anteil der Unproduktiven auf ein vertretbares Maß zu beschränken.

Die geringe Leistungsfähigkeit der Institute auf den Plätzen $7-13$ erklärt sich wahrscheinlich aus der Kombination aller oben angeführten Gründe. Speziell sind alle diese Institute relativ klein. Mit Ausnahme von Wirtschaftsuniversität (WU) und Wifo hat keines dieser Institute mehr als 20 angestellte Forscher. Das Wifo sieht allerdings seine Hauptaufgabe nicht in der Art von Forschung, die zu Publikationen in internationalen Journalen führt. Entsprechend ist es beim Wifo auch nicht eine zu geringe Anzahl von Publikationen, die den Erfolg verhindert, sondern die geringen SSCI-Impact-Faktoren.

\footnotetext{
${ }^{5}$ Eine quantitative Erhebung des Zusammenhangs zwischen Studentenzahlen und Forschungsleistung steht leider noch aus.
} 


\subsection{Individuelle Leistungen}

Ein Nebenprodukt der vorliegenden Studie ist eine vollständige Erfassung aller in Österreich an volkswirtschaftlichen Instituten tätigen Forscher nach ihren Impacts. Dies erlaubt es, auch die individuellen Leistungen zu betrachten. Zwar haben verschiedene Arbeiten ein und desselben Autors oft unterschiedliche Bedeutung für den Fortschritt der Wissenschaft, sodass ein genaues Ranking der Individuen die Zitationen der Autoren, und nicht der Zeitschriften, zu erfassen hätte. Andererseits wird in der Literatur das hier verwendete Maß auch für individuelle Rankings benutzt (z.B. Bommer und Ursprung (1998), Coupé (2000), Baltagi (1999)). Und da hier die individuellen Leistungen nur statistisch erfasst werden, ist die erwähnte Einschränkung von untergeordneter Bedeutung.

\section{(Abbildung 1 etwa hier einfügen.)}

Abbildung 1 gibt die Verteilung der individuellen Impact-Faktoren graphisch wider. Dabei fällt der hohe Anteil der Unproduktiven auf: Von den 177 ausgewerteten Personen haben im untersuchten Zeitraum 112 Personen (oder 63 Prozent) keine Arbeit mit positivem Impact-Faktor veröffentlicht. Anders ausgedrückt: Nur etwa ein Drittel aller in Österreich angestellten Forscher haben in einem Zeitraum von zehn Jahren einen messbaren Einfluss auf den Fortschritt der Wissenschaft gehabt. Und dies obwohl 56 Prozent aller erfassten Personen (ordentliche oder außerordentliche) Professoren sind. Der geringe Anteil der Produktiven ist also kaum damit zu erklären, dass sich in der Grundgesamtheit zu viele Personen am Anfang ihrer Karriere befinden.

Ein individuelles Rankings zeigt außerdem, dass drei der "Top-5" keine ordentlichen Professoren sind. Um dies genauer zu beleuchten, wurden die Daten nach der Position der Individuen (ordentlicher Professor, außerordentlicher Professor, oder Assistent) ausgewertet. Tabelle 3 gibt (in der dritten und vierten Spalte) die durchschnittlichen Impact-Faktoren und den Anteil der Unproduktiven in den drei derart gebildeten Gruppen wider. ${ }^{6}$ Wie zu erwarten, haben die ordentlichen Professoren den höchsten durchschnittlichen Impact. Den höchsten Anteil an Unproduktiven hat die Gruppe der Assistenten, da sich in dieser Gruppe viele am Beginn ihrer Karriere befinden. Den zweithöchsten Anteil an Unproduktiven weist aber die Gruppe der ordentlichen Professoren auf. Dies könnte ein Hinweis auf eine Überalterung sein.

\footnotetext{
${ }^{6}$ In die Gruppe "ao.Prof." wurden auch die beiden Abteilungsleiter des IHS einbezogen, und in die Gruppe "o.Prof." alle Honorar-Professoren der Grundgesamtheit.
} 


\section{(Tabelle 3 etwa hier einfügen.)}

Angesichts der ausgeprägten Schiefe der Verteilung der Impact-Faktoren stellt sich natürlich die Frage nach der Aussagekraft der in Tabelle 3 angegebenen durchschnittlichen Impact-Faktoren nach Positionen. Tatsächlich ist die Ausagekraft gering. Ein einfacher $\chi^{2}$-Test der Hypothese, dass die Stichprobe der ordentlichen Professoren eine reine Zufallsstichprobe aus der Grundgesamtheit darstellt, erlaubt es nicht, diese Hypothese abzulehnen (bei einem Signifikanzniveau von 5 Prozent; $\chi^{2}$-Verteilung mit 5 Freiheitsgraden). Dasselbe gilt für die außerordentlichen Professoren. Nur für die Gruppe der Assistenten wird die Hypothese, es handle sich um eine Zufallsstichprobe, abgelehnt (bei einem Signifikanzniveau von 5 Prozent). Diese Gruppe weicht also als einzige signifikant von der Verteilung der Grundgesamtheit ab. Dass die Forschungsproduktivität der Assistenten signifikant niedriger ist, sollte nicht überraschen. Zwar sollten die jungen Jahre eigentlich die produktivsten sein, aber im herrschenden System werden Assistenten zu stark für Lehraufgaben herangezogen. Das, und dass viele in dieser Gruppe erst während des Beobachtungszeitraums ihre Arbeit aufgenommen haben, erklärt diesen Befund.

\section{Schlussfolgerungen}

In der vorliegenden Studie werden die universitäten und außeruniversitären volkswirtschaftlichen Institute Österreichs, zuzüglich der entsprechenden Institute an der London School of Economics, der Universität Bonn und der Universität Mannheim, hinsichtlich ihres Beitrags zur Forschung nach dem holländischen System im Zeitraum 1990-99 verglichen. Wie durch internationale Studien nahegelegt, ergeben sich drei wichtige Befunde. Erstens bleibt der deutschsprachige Raum hinter der internationalen Spitze zurück. Zweitens hat Österreich drei der Spitzeninstitute des deutschsprachigen Raums vorzuweisen: Die Universität Wien, das Institut für Höhere Studien und die Universität Linz verfügen über leistungsfähige volkswirtschaftliche Institute. Drittens, zwischen dieser Spitzengruppe und den restlichen österreichischen Instituten lässt sich ein sehr großer Abstand festzustellen.

Mit der vorliegenden Studie wird eine exakte quantitative Grundlage für die öffentliche Diskussion um die Qualität der Forschung in Österreich geschaffen zumindest für den Bereich der Volkswirtschaftslehre. Nur wenn derartige, auf Fakten statt Vermutungen beruhende Informationen in die Diskussion eingehen, hat Österreich eine Chance, vernünftige Forschungspolitik zu betreiben. 


\section{References}

[1] Badi H. Baltagi (1999): “Applied Econometrics Ranking: 1989-1995”, Journal of Applied Econometrics 14, 423-441.

[2] Luc Bauwens, Alan Kirman, Michel Lubrano und Camelia Protopopescu (2000): "Some Methodological Aspects for Ranking European Economic Departments: a Statistical Approach", mimeo, Universität Marseille (http://durandal.cnrs-mrs.fr/PP/lubrano/lubrano.htm).

[3] Rolf Bommer und Heinrich W. Ursprung (1998): "Spieglein, Spieglein an der Wand - Eine publikationsanalytische Erfassung der Forschungsleistungen volkswirtschaftlicher Fachbereiche in Deutschland, Österreich und der Schweiz", Zeitschrift für Wirtschafts- und Sozialwissenschaften 118, 1-28.

[4] Tom Coupé (2000): "Revealed Performances: World Wide Rankings of Economists and Economic Departments", mimeo, Freie Universität of Brüssel (http://student.ulb.ac.be/ tcoupe/rank1000.html).

[5] Pantelis Kalaitzidakis, Theofanis Mammuneas und Thanasis Stengos (1999): "European Economics: An analysis based on publications in the core journals", European Economic Review 43, 1150-1168.

[6] Pantelis Kalaitzidakis, Theofanis Mammuneas und Thanasis Stengos (2001): "Ranking of Academic Journals and Institutions in Economics", mimeo, erscheint in: European Economic Review.

[7] David Laband und Michael Piette (1994): "The Relative Impact of Economic Journals", Journal of Economic Literature 32, 640-666. 


\section{Appendix}

Liste der ausgewerteten Institute, nach Universitäten bzw. außeruniversitären Einrichtungen geordnet:

- Wirtschaftswissenschaftlicher Fachbereich, Rheinische Friedrich-WilhelmsUniversität Bonn;

- Institut für Finanzwissenschaft und Öffentliche Wirtschaft, Karl-FranzensUniversität Graz;

- Institut für Volkswirtschaftslehre, Karl-Franzens-Universität Graz;

- Institut für Finanzwissenschaft, Leopold-Franzens-Universität Innsbruck;

- Institut für Wirtschaftstheorie, Wirtschaftspolitik und Wirtschaftsgeschichte, Leopold-Franzens-Universität Innsbruck;

- Abteilung für Volkswirtschaftstheorie und -politik (VWL1), Universität Klagenfurt;

- Institut für Volkswirtschaftslehre, Johannes-Kepler-Universität Linz;

- Department of Economics, London School of Economics and Political Science;

- Fakultät für Volkswirtschaftslehre, Universität Mannheim;

- Institut für Wirtschaftswissenschaften, Universität Salzburg;

- Department of Economics and Finance, Institut für Höhere Studien, Wien;

- Institut für Volkswirtschaftslehre und Wirtschaftsinformatik, Technische Universität Wien;

- Institut für Wirtschaftswissenschaften, Universität Wien;

- Wirtschaftsforschungsinstitut, Wien;

- Forschungsinstitut für Europafragen/Fachbereich VWL, Wirtschaftsuniversität Wien;

- Institut für Finanzwissenschaft, Wirtschaftsuniversität Wien;

- Institut für Volkswirtschaftstheorie und -politik, Wirtschaftsuniversität Wien. 
Tabelle 1

\begin{tabular}{|c|c|c|c|c|c|c|c|}
\hline \multicolumn{2}{|c|}{$\begin{array}{c}\text { Studie } \\
\text { Zeitraum } \\
\text { \# gereihter Inst. } \\
\text { \# verwend. Jour. } \\
\text { Größenkorr. } \\
\text { Reichweite }\end{array}$} & \multicolumn{2}{|c|}{$\begin{array}{c}\text { EER 99 } \\
1991-96 \\
198 \\
10 \\
\text { nein } \\
\text { Europa }\end{array}$} & \multicolumn{2}{|c|}{$\begin{array}{c}\text { EER 01 } \\
1994-98 \\
200 \\
30 \\
\text { nein } \\
\text { Europa } \\
\end{array}$} & \multicolumn{2}{|c|}{$\begin{array}{c}\mathrm{B}+\mathrm{U} \\
1990-95 \\
71 \\
196 \\
\text { ja } \\
\text { GER,AUT,CH }\end{array}$} \\
\hline Inst. & Nat. & Prod. & Rang & Prod. & Rang & Prod. & Rang \\
\hline LSE & UK & 397 & 1 & 549 & 2 & n.a. & n.a. \\
\hline U Wien & AUT & 110 & 11 & 208 & 19 & 16.8 & 2 \\
\hline U Bonn & GER & 82 & 18 & 202 & 20 & 13.4 & 4 \\
\hline TU Wien & AUT & 38 & 40 & 30 & 91 & 15.8 & 3 \\
\hline IHS & AUT & 23 & 61 & n.a. & n.a. & n.a. & n.a. \\
\hline U Mannheim & GER & 11 & 94 & 58 & 60 & 10.6 & 8 \\
\hline WU Wien & AUT & 4 & 129 & n.a. & n.a. & 0.5 & 69 \\
\hline U Linz & AUT & 0.9 & 173 & 23 & 105 & 11.6 & 6 \\
\hline U Innsbruck & AUT & 0.6 & 177 & n.a. & n.a. & 1.9 & 44 \\
\hline U Graz & AUT & n.a. & n.a. & n.a. & n.a. & 4.1 & 27 \\
\hline Wifo & AUT & n.a. & n.a. & n.a. & n.a. & n.a. & n.a. \\
\hline U Klagenfurt & AUT & n.a. & n.a. & n.a. & n.a. & n.a. & n.a. \\
\hline U Salzburg & AUT & n.a. & n.a. & n.a. & n.a. & n.a. & n.a. \\
\hline
\end{tabular}

Quellen: $\quad$ EER 99 = Kalaitzidakis et.al. (1999);

EER 01 = Kalaitzidakis et.al. (2001);

$\mathrm{B}+\mathrm{U}=$ Bommer und Ursprung (1998). 
Tabelle 2

\begin{tabular}{|c|c|c|c|}
\hline Inst. & Nat. & Impact & Rang \\
\hline \hline LSE & UK & 2.953 & 1 \\
\hline U Wien & AUT & 1.195 & 2 \\
\hline IHS & AUT & 1.110 & 3 \\
\hline U Bonn & GER & 0.911 & 4 \\
\hline U Mannheim & GER & 0.887 & 5 \\
\hline U Linz & AUT & 0.842 & 6 \\
\hline Wifo & AUT & 0.225 & 7 \\
\hline U Graz & AUT & 0.219 & 8 \\
\hline U Innsbruck & AUT & 0.167 & 9 \\
\hline TU Wien & AUT & 0.152 & 10 \\
\hline WU Wien & AUT & 0.088 & 11 \\
\hline U Klagenfurt & AUT & 0.048 & 12 \\
\hline U Salzburg & AUT & 0.000 & 13 \\
\hline
\end{tabular}


Tabelle 3

\begin{tabular}{|c|c|c|c|}
\hline Pos. & Anz. & Impact & \% Unprod. \\
\hline \hline o.Prof. & 53 & 0.71 & 45 \\
\hline ao.Prof. & 46 & 0.51 & 43 \\
\hline Ass. & 78 & 0.10 & 75 \\
\hline
\end{tabular}




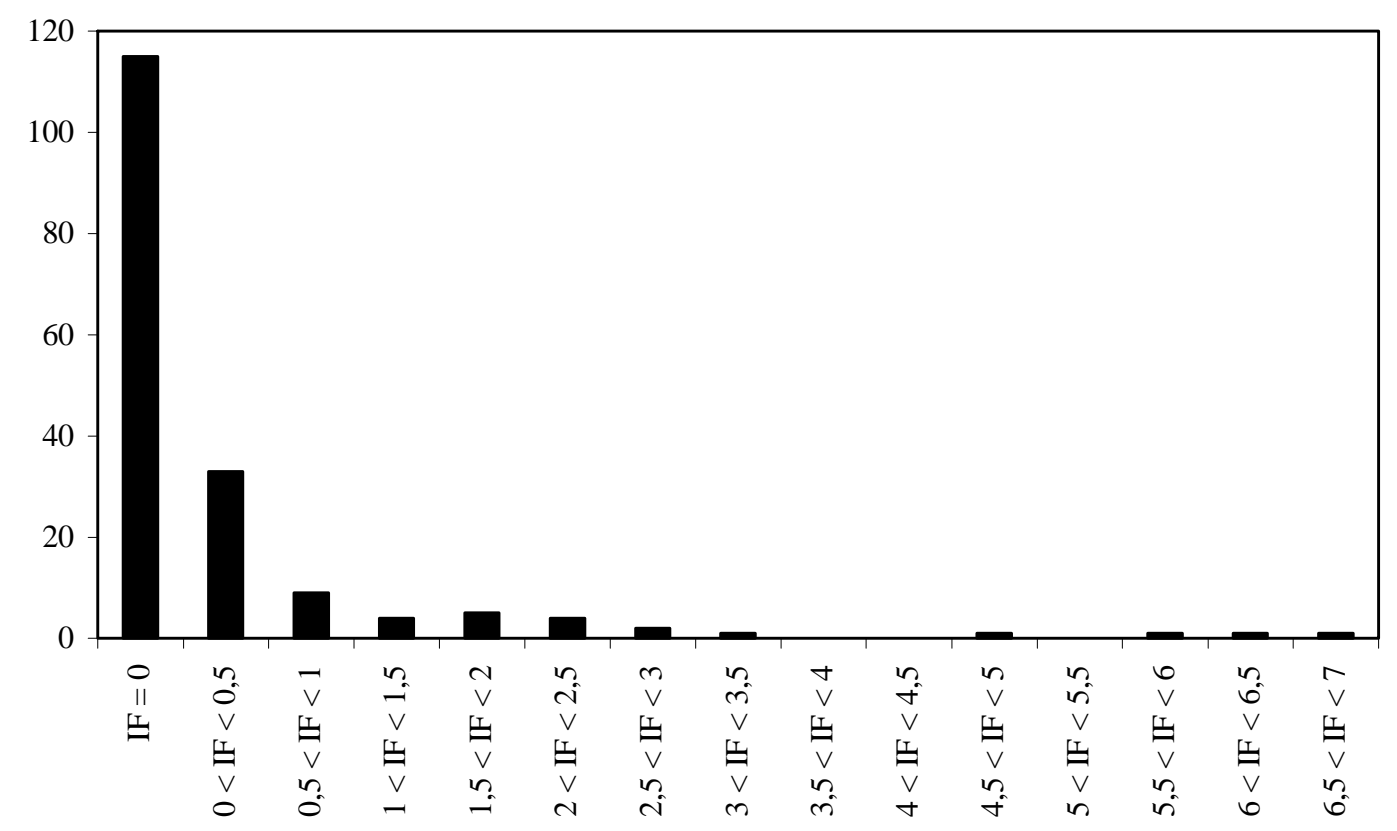

Abbildung 1: Verteilung der individuellen Impact-Faktoren 


\begin{tabular}{|l|l|}
\hline Institut & Universität \\
\hline Institut für Volkswirtschaftslehre & Johannes-Kepler-Universität Linz \\
\hline Department of Economics & London School of Economics and Political Science \\
\hline Wirtschaftswissenschaftlicher Fachbereich & Rheinische Friedrich-Wilhelms-Universität Bonn \\
\hline Institut für Volkswirtschaftslehre u. Wirtschaftsinformatik & Technische Universität Wien \\
\hline Institut für Finanzwissenschaft u. Öffentliche Wirtschaft & Karl-Franzens-Universität Graz \\
\hline Institut für Volkswirtschaftslehre & Karl-Franzens-Universität Graz \\
\hline Institut für Wirtschaftstheorie, Wirtschaftspolitik u. Wirtschaftsgeschichte & Leopold-Franzens-Universität Innsbruck \\
\hline Institut für Finanzwissenschaft & Leopold-Franzens-Universität Innsbruck \\
\hline Abteilung für Volkswirtschaftstheorie und -politik (VWL1) & Universität Klagenfurt \\
\hline Fakultät für Volkswirtschaftslehre & Universität Mannheim \\
\hline Institut für Wirtschaftswissenschaften & Universität Salzburg \\
\hline Institut für Wirtschaftswissenschaften & Universität Wien \\
\hline Wirtschaftsforschungsinstitut & Wirtschaftsforschungsinstitut \\
\hline Volkswirtschftstheorie und -politik & Wirtschaftsuniversität Wien \\
\hline Forschungsinstitut für Europafragen/Fachbereich VWL & Wirtschaftsuniversität Wien \\
\hline Institut für Finanzwissenschaft & Wirtschaftsuniversität Wien \\
\hline Department of Economics and Finance (Eco\&Fin) & Institut für Höhere Studien, Wien \\
\hline
\end{tabular}


Universität Linz

\begin{tabular}{|c|c|c|c|c|c|c|c|c|c|c|c|}
\hline & Author(s) & Journal & & & & & $\begin{array}{c}\text { Impact } \\
\text { Factor }\end{array}$ & $\begin{array}{r}\begin{array}{r}\text { Seiten } \\
\text { korr. }\end{array} \\
\end{array}$ & Autoren & Impact & $\begin{array}{r}\text { personal } \\
\text { Impact }\end{array}$ \\
\hline $\begin{array}{r}\text { Efficiency and } \\
\text { Effectiveness Control } \\
\text { Based on Economic } \\
\text { Analysis: The Example of } \\
\text { the Austrian Court of Audit }\end{array}$ & $\begin{array}{r}\text { Bartel,--Rainer; } \\
\text { Schneider,- } \\
\text { Friedrich }\end{array}$ & $\begin{array}{r}\text { European- } \\
\text { Journal-of- } \\
\text { Law-and- } \\
\text { Economics; } \\
1(4), \\
\text { December } \\
\text { 1994, pages } \\
237-62 . \\
\end{array}$ & 1994 & Bartel & Rainer & $U \operatorname{Lin} z$ & 0 & 1 & 2 & 0 & \\
\hline $\begin{array}{r}\text { Suggestions for Effective } \\
\text { Public Auditing }\end{array}$ & $\begin{array}{r}\text { Bartel,-Rainer; } \\
\text { van- } \\
\text { Rietschoten,- } \\
\text { Kees }\end{array}$ & $\begin{array}{r}\text { Annals-of- } \\
\text { Public-and- } \\
\text { Cooperative- } \\
\text { Economics; } \\
65(2), 1994, \\
\text { pages } 227-46 .\end{array}$ & 1994 & Bartel & Rainer & $U \operatorname{Lin} z$ & 0 & 1 & 2 & 0 & \\
\hline $\begin{array}{l}\text { The "Mess" of the Public } \\
\text { Industrial Production in } \\
\text { Austria: A Typical Case of } \\
\text { Public Sector Inefficiency? }\end{array}$ & $\begin{array}{r}\text { Bartel,--Rainer; } \\
\text { Schneider,- } \\
\text { Friedrich }\end{array}$ & $\begin{array}{r}\text { Public-Choice; } \\
68(1-3), \\
\text { January } 1991 \\
\text { pages } 17-40 .\end{array}$ & 1991 & Bartel & Rainer & U Linz & 0,43 & 1 & 2 & 0,2866 & R. Bartel: \\
\hline $\begin{array}{r}\text { Betriebsgrosse und } \\
\text { Effizienz in der } \\
\text { oberosterreichischen } \\
\text { idustrie. Erste empirische } \\
\text { Ergebnisse. }\end{array}$ & Bartel,--Rainer & $\begin{array}{r}\text { Zeitschrift-fur- } \\
\text { Betriebswirtsc } \\
\text { haft; } 60(7), \\
\text { July } 1990, \\
\text { pages } 665-84 .\end{array}$ & 1990 & Bartel & Rainer & U Linz & 0 & 1 & 1 & 0 & $\begin{array}{r}0,2866666 \\
6\end{array}$ \\
\hline $\begin{array}{r}\text { Measuring the Severity of } \\
\text { Unemployment }\end{array}$ & $\begin{array}{r}\text { Riese,-Martin; } \\
\text { Brunner,-- } \\
\text { Johann-K. }\end{array}$ & $\begin{array}{r}\text { Journal-of- } \\
\text { Economics- } \\
\text { (Zeitschrift-fur- } \\
\text { Nationalokono } \\
\text { mie); } 67(2), \\
1998, \text { pages } \\
167-80 . \\
\end{array}$ & 1998 & Brunner & Johann & $U \operatorname{Lin} z$ & 0,18 & 1 & 2 & 0,12 & \\
\hline $\begin{array}{l}\text { Transition from a Pay-As- } \\
\text { You-Go to a Fully Funded } \\
\text { 'ension System: The Case } \\
\text { of Differing Individuals and } \\
\text { Intragenerational Fairness }\end{array}$ & $\begin{array}{l}\text { Brunner,-- } \\
\text { Johann-K. }\end{array}$ & $\begin{array}{r}\text { Journal-of- } \\
\text { Public- } \\
\text { Economics; } \\
60(1), \text { April } \\
\text { 1996, pages } \\
131-46 . \\
\end{array}$ & 1996 & Brunner & Johann & U Linz & 0,82 & 1 & 1 & 0,82 & \\
\hline
\end{tabular}




\begin{tabular}{|c|c|c|c|c|c|c|c|c|c|c|c|}
\hline $\begin{array}{r}\text { A Theorem on Utilitarian } \\
\text { Redistribution }\end{array}$ & $\begin{array}{l}\text { Brunner,-- } \\
\text { Johann-K. }\end{array}$ & $\begin{array}{r}\text { Social-Choice- } \\
\text { and-Welfare; } \\
\text { 12(2), March } \\
\text { 1995, pages } \\
175-79 .\end{array}$ & 1995 & Brunner & Johann & $U \operatorname{Lin} z$ & 0,55 & 0,5 & 1 & 0,275 & \\
\hline $\begin{array}{l}\text { Redistribution and the } \\
\text { Efficiency of the Pay-as- } \\
\text { You-Go Pension System }\end{array}$ & $\begin{array}{l}\text { Brunner,- } \\
\text { Johann-K. }\end{array}$ & $\begin{array}{r}\text { Journal-of- } \\
\text { Institutional- } \\
\text { and- } \\
\text { Theoretical- } \\
\text { Economics; } \\
150(3), \text { Sept. } \\
\text { 1994, pp. } 511- \\
23 .\end{array}$ & 1994 & Brunner & Johann & U Linz & 0,29 & 1 & 1 & 0,29 & \\
\hline $\begin{array}{r}\text { A Note on the Optimum } \\
\text { Income Tax }\end{array}$ & $\begin{array}{l}\text { Brunner,- } \\
\text { Johann-K. }\end{array}$ & $\begin{array}{r}\text { Journal-of- } \\
\text { Public- } \\
\text { Economics; } \\
50(3), \text { March } \\
\text { 1993, pages } \\
445-51 .\end{array}$ & 1993 & Brunner & Johann & U Linz & 0,82 & 0,5 & 1 & 0,41 & J. Brunner: \\
\hline $\begin{array}{r}\text { The Principle of Equal } \\
\text { Proportional Sacrifice as a } \\
\text { Bargaining Solution }\end{array}$ & $\begin{array}{r}\text { Brunner,- } \\
\text { Johann-K. }\end{array}$ & $\begin{array}{r}\text { Bulletin-of- } \\
\text { Economic- } \\
\text { Research; } \\
\text { 45(2), April } \\
\text { 1993, pages } \\
\text { 133-46. }\end{array}$ & 1993 & Brunner & Johann & U Linz & 0 & 1 & 1 & 0 & 1,915 \\
\hline $\begin{array}{r}\text { Raising Children: Private } \\
\text { Expenditures and } \\
\text { Foregone Earnings }\end{array}$ & $\begin{array}{r}\text { Buchegger,- } \\
\text { Reiner; } \\
\text { Zweimuller,- } \\
\text { Josef }\end{array}$ & $\begin{array}{r}\text { Labour; } 6(1) \\
\text { Spring } 1992, \\
\text { pages } 181- \\
200 .\end{array}$ & 1992 & Buchegger & Reiner & U Linz & 0 & 1 & 2 & 0 & $\begin{array}{r}\mathrm{R} \text {. } \\
\text { Buchegger: } \\
0\end{array}$ \\
\hline $\begin{array}{l}\text { On the Gap between } \\
\text { 'ayment Card and Closed- } \\
\text { Ended CVM-Answers }\end{array}$ & $\begin{array}{r}\text { Hackl,-Franz; } \\
\text { Pruckner,- } \\
\text { Gerald-J. }\end{array}$ & $\begin{array}{r}\text { Applied- } \\
\text { Economics; } \\
31(6), \text { June } \\
\text { 1999, pages } \\
\text { 733-42. }\end{array}$ & 1999 & Hackl & Franz & $U \operatorname{Linz}$ & 0,28 & 0,5 & 2 & 0,0933 & \\
\hline $\begin{array}{r}\text { Towards More Efficient } \\
\text { Compensation } \\
\text { Programmes for Tourists' } \\
\text { 3enefits from Agriculture in } \\
\text { Europe }\end{array}$ & $\begin{array}{r}\text { Hackl,-Franz; } \\
\text { Pruckner,- } \\
\text { Gerald-J. }\end{array}$ & $\begin{array}{r}\text { Environmental } \\
\text {-and- } \\
\text { Resource- } \\
\text { Economics; } \\
10(2), \\
\text { September }\end{array}$ & 1997 & Hackl & Franz & U Linz & 0 & 1 & 2 & 0 & \\
\hline
\end{tabular}




\begin{tabular}{|c|c|c|c|c|c|c|c|c|c|c|c|}
\hline & & $\begin{array}{c}\text { 1997, pages } \\
189-205 .\end{array}$ & & & & & & & & & \\
\hline $\begin{array}{l}\text { A Fair Mechanism for } \\
\text { Efficient Reduction of } \\
\text { Global CO2-Emissions }\end{array}$ & $\begin{array}{r}\text { Falkinger,- } \\
\text { Josef; Hackl,- } \\
\text { Franz; } \\
\text { Pruckner,- } \\
\text { Gerald-J. }\end{array}$ & $\begin{array}{l}\text { FinanzArchiv; } \\
53(3-4), 1996, \\
\text { pages 308-31. }\end{array}$ & 1996 & Hackl & Franz & U Linz & 0 & 1 & 3 & 0 & F. Hackl: \\
\hline $\begin{array}{r}\text { inanzinnovationen und die } \\
\text { LM-Kurve. }\end{array}$ & Hackl,-Franz & $\begin{array}{r}\text { Kredit-und- } \\
\text { Kapital; } 26(3), \\
\text { 1993, pages } \\
362-74 . \\
\end{array}$ & 1993 & Hackl & Franz & $U \operatorname{Lin} z$ & 0 & 1 & 1 & 0 & 0,093 \\
\hline $\begin{array}{r}\text { Commercial Policy and } \\
\text { Jynamic Adjustment under } \\
\text { Monopolistic Competition }\end{array}$ & $\begin{array}{r}\text { Keuschnigg,- } \\
\text { Christian; } \\
\text { Kohler,-Wilhelm }\end{array}$ & $\begin{array}{r}\text { Journal-of- } \\
\text { International- } \\
\text { Economics; } \\
\text { 40(3-4), May } \\
\text { 1996, pages } \\
373-409 .\end{array}$ & 1996 & Kohler & Wilhelm & $U \operatorname{Lin} z$ & 0,8 & 1 & 2 & 0,5333 & \\
\hline $\begin{array}{r}\text { Austria in the European } \\
\text { Union: Dynamic Gains } \\
\text { from Integration and } \\
\text { Distributional Implications }\end{array}$ & $\begin{array}{r}\text { Keuschnigg,- } \\
\text { Christian; } \\
\text { Kohler,-Wilhelm }\end{array}$ & $\begin{array}{r}\text { Economic- } \\
\text { Policy:-A- } \\
\text { European- } \\
\text { Forum; 0(22), } \\
\text { April 1996, } \\
\text { pages } 155-90 .\end{array}$ & 1996 & Kohler & Wilhelm & $U \operatorname{Lin} z$ & 0 & 1 & 2 & 0 & \\
\hline $\begin{array}{r}\text { Factor-Price Equalization } \\
\text { under Joint and Nonjoint } \\
\text { Production }\end{array}$ & $\begin{array}{r}\text { Albert,-Max; } \\
\text { Kohler,-Wilhelm }\end{array}$ & $\begin{array}{r}\text { Journal-of- } \\
\text { Economics- } \\
\text { (Zeitschrift-fur- } \\
\text { Nationalokono } \\
\text { mie); } 62(3), \\
1995, \text { pages } \\
271-94 .\end{array}$ & 1995 & Kohler & Wilhelm & U Linz & 0,18 & 1 & 2 & 0,12 & \\
\hline $\begin{array}{r}\text { Dynamic Effects of Tariff } \\
\text { Liberalization: An } \\
\text { Intertemporal CGE } \\
\text { Approach }\end{array}$ & $\begin{array}{r}\text { Keuschnigg,- } \\
\text { Christian; } \\
\text { Kohler,-Wilhelm }\end{array}$ & $\begin{array}{r}\text { Review-of- } \\
\text { International- } \\
\text { Economics; } \\
3(1), \text { February } \\
\text { 1995, pages } \\
20-35 .\end{array}$ & 1995 & Kohler & Wilhelm & U Linz & 0 & 1 & 2 & 0 & \\
\hline $\begin{array}{l}\text { Modeling Intertemporal } \\
\text { General Equilibrium: An } \\
\text { Application to Austrian } \\
\text { Commercial Policy }\end{array}$ & $\begin{array}{r}\text { Keuschnigg,- } \\
\text { Christian; } \\
\text { Kohler,-Wilhelm }\end{array}$ & $\begin{array}{r}\text { Empirical- } \\
\text { Economics; } \\
19(1), 1994, \\
\text { pages } 131-64 .\end{array}$ & 1994 & Kohler & Wilhelm & U Linz & 0 & 1 & 2 & 0 & \\
\hline
\end{tabular}




\begin{tabular}{|c|c|c|c|c|c|c|c|c|c|c|c|}
\hline $\begin{array}{r}\text { Multilateral Trade } \\
\text { Liberalization: Some } \\
\text { General Equilibrium } \\
\text { Simulation Results for } \\
\text { Austria }\end{array}$ & Kohler,-Wilhelm & $\begin{array}{r}\text { Empirica; } \\
18(2), 1991 \\
\text { pages 167-99. }\end{array}$ & 1991 & Kohler & Wilhelm & U Linz & 0 & 1 & 1 & 0 & \\
\hline $\begin{array}{r}\text { How Robust Are Sign and } \\
\text { Rank Order Tests of the } \\
\text { Heckscher-Ohlin-Vanek } \\
\text { Theorem? }\end{array}$ & Kohler,-Wilhelm & $\begin{array}{r}\text { Oxford- } \\
\text { Economic- } \\
\text { Papers,-N.-S.; } \\
\text { 43(1), January } \\
\text { 1991, pages } \\
158-71 .\end{array}$ & 1991 & Kohler & Wilhelm & $U \operatorname{Linz}$ & 0,74 & 1 & 1 & 0,74 & W. Kohler: \\
\hline $\begin{array}{r}\text { Income Distribution and } \\
\text { Labor Market Effects of } \\
\text { Austrian Pre- and Post- } \\
\text { Tokyo-Round Tariff } \\
\text { Protection }\end{array}$ & Kohler,-Wilhelm & $\begin{array}{r}\text { European- } \\
\text { Economic- } \\
\text { Review; 35(1), } \\
\text { January 1991, } \\
\text { pages 139-54. }\end{array}$ & 1991 & Kohler & Wilhelm & $U \operatorname{Lin} z$ & 0,84 & 1 & 1 & 0,84 & $\begin{array}{r}2,2333333 \\
3\end{array}$ \\
\hline $\begin{array}{r}\text { Vertical Product } \\
\text { Differentiation in EU } \\
\text { Markets: The Relative } \\
\text { Position of East European } \\
\text { Producers }\end{array}$ & $\begin{array}{r}\text { Landesmann,-- } \\
\text { Michael; } \\
\text { Burgstaller,- } \\
\text { Johann }\end{array}$ & $\begin{array}{r}\text { Russian-and- } \\
\text { East- } \\
\text { European- } \\
\text { Finance-and- } \\
\text { Trade; 34(1), } \\
\text { Jan.-Feb. } \\
\text { 1998, pages } \\
32-78 .\end{array}$ & 1998 & Landesmann & Michael & U Linz & 0 & 1 & 2 & 0 & \\
\hline $\begin{array}{r}\text { rom Theory into Practice? } \\
\text { Restructuring and } \\
\text { Dynamism in Transition } \\
\text { Economies }\end{array}$ & $\begin{array}{r}\text { Carlin,-Wendy; } \\
\text { Landesmann,- } \\
\text { Michael }\end{array}$ & $\begin{array}{r}\text { Oxford- } \\
\text { Review-of- } \\
\text { Economic- } \\
\text { Policy; 13(2), } \\
\text { Summer 1997, } \\
\text { pages 77-105. }\end{array}$ & 1997 & Landesmann & Michael & U Linz & 0,29 & 1 & 2 & 0,1933 & \\
\hline $\begin{array}{r}\text { Balance-of-Payments } \\
\text { Constrained Growth in } \\
\text { Central and Eastern } \\
\text { Europe and Scenarios of } \\
\text { East-West Integration }\end{array}$ & $\begin{array}{r}\text { Landesmann,- } \\
\text { Michael; } \\
\text { Poschl,-Josef }\end{array}$ & $\begin{array}{r}\text { Russian-and- } \\
\text { East- } \\
\text { European- } \\
\text { Finance-and- } \\
\text { Trade; 32(6), } \\
\text { Nov.-Dec. } \\
\text { 1996, pages } \\
30-84 .\end{array}$ & 1996 & Landesmann & Michael & U Linz & 0 & 1 & 2 & 0 & $\begin{array}{r}\mathrm{M} . \\
\text { Landesma } \\
\mathrm{nn}:\end{array}$ \\
\hline $\begin{array}{r}\text { Institutions and Economic } \\
\text { Change }\end{array}$ & $\begin{array}{r}\text { Landesmann,-- } \\
\text { Michael-A.; }\end{array}$ & $\begin{array}{r}\text { Structural- } \\
\text { Change-and- }\end{array}$ & 1994 & Landesmann & Michael & U Linz & 0 & 0,5 & 2 & 0 & $\begin{array}{r}0,1933333 \\
3\end{array}$ \\
\hline
\end{tabular}




\begin{tabular}{|c|c|c|c|c|c|c|c|c|c|c|c|}
\hline & Pagano,-Ugo & $\begin{array}{r}\text { Economic- } \\
\text { Dynamics; } \\
5(2), \\
\text { December } \\
1994, \text { pages } \\
199-203 .\end{array}$ & & & & & & & & & \\
\hline $\begin{array}{r}\text { Towards More Efficient } \\
\text { Compensation } \\
\text { Programmes for Tourists' } \\
\text { 3enefits from Agriculture in } \\
\text { Europe }\end{array}$ & $\begin{array}{r}\text { Hackl,-Franz; } \\
\text { Pruckner,-- } \\
\text { Gerald-J. }\end{array}$ & $\begin{array}{r}\text { Environmental } \\
\text {-and- } \\
\text { Resource- } \\
\text { Economics; } \\
10(2), \\
\text { September } \\
\text { 1997, pages } \\
189-205 .\end{array}$ & 1997 & Pruckner & Gerald & $\cup \operatorname{Lin} z$ & 0 & 1 & 2 & 0 & \\
\hline $\begin{array}{l}\text { Environmental Valuation-- } \\
\text { To Use or Not to Use? A } \\
\text { Comparative Study of the } \\
\text { United States and Europe }\end{array}$ & $\begin{array}{r}\text { Navrud,-Stale; } \\
\text { Pruckner,- } \\
\text { Gerald-J. }\end{array}$ & $\begin{array}{r}\text { Environmental } \\
\text {-and- } \\
\text { Resource- } \\
\text { Economics; } \\
10(1) \text {, July } \\
\text { 1997, pages } \\
1-26 .\end{array}$ & 1997 & Pruckner & Gerald & $U \operatorname{Lin} z$ & 0 & 1 & 2 & 0 & \\
\hline $\begin{array}{r}\text { A Fair Mechanism for } \\
\text { Efficient Reduction of } \\
\text { Global CO2-Emissions } \\
\end{array}$ & $\begin{array}{r}\text { Falkinger,-J.; } \\
\text { Hackl,-F.; } \\
\text { Pruckner,-G.-J. }\end{array}$ & $\begin{array}{l}\text { FinanzArchiv; } \\
53(3-4), 1996, \\
\text { pages 308-31. }\end{array}$ & 1996 & Pruckner & Gerald & $U \operatorname{Linz}$ & 0 & 1 & 3 & 0 & $\begin{array}{r}\text { G. } \\
\text { Pruckner: }\end{array}$ \\
\hline $\begin{array}{l}\text { Agricultural Landscape } \\
\text { Cultivation in Austria: An } \\
\text { Application of the CVM }\end{array}$ & $\begin{array}{r}\text { Pruckner,- } \\
\text { Gerald-J. }\end{array}$ & $\begin{array}{r}\text { European- } \\
\text { Review-of- } \\
\text { Agricultural- } \\
\text { Economics; } \\
22(2), 1995, \\
\text { pages } 173-90 .\end{array}$ & 1995 & Pruckner & Gerald & $U \operatorname{Lin} z$ & 0,32 & 1 & 1 & 0,32 & 0,32 \\
\hline $\begin{array}{r}\text { Effective Demand versus } \\
\text { Profit Maximization in } \\
\text { tggregate Demand/Supply } \\
\text { Analysis: A Dynamic } \\
\text { Perspective }\end{array}$ & $\begin{array}{r}\text { Bhaduri,-Amit; } \\
\text { Laski,- } \\
\text { Kazimierz; } \\
\text { Riese,-Martin }\end{array}$ & $\begin{array}{r}\text { Banca- } \\
\text { Nazionale-del- } \\
\text { Lavoro- } \\
\text { Quarterly- } \\
\text { Review; } \\
\text { 52(210), Sept. } \\
\text { 1999, pp. } 281- \\
93 . \\
\end{array}$ & 1999 & Riese & Martin & $U \operatorname{Lin} z$ & 0 & 1 & 3 & 0 & \\
\hline $\begin{array}{r}\text { Measuring the Severity of } \\
\text { Unemployment }\end{array}$ & $\begin{array}{r}\text { Riese,-Martin; } \\
\text { Brunner,- }\end{array}$ & $\begin{array}{r}\text { Journal-of- } \\
\text { Economics- }\end{array}$ & 1998 & Riese & Martin & U Linz & 0,18 & 1 & 2 & 0,12 & M. Riese: \\
\hline
\end{tabular}




\begin{tabular}{|c|c|c|c|c|c|c|c|c|c|c|c|}
\hline & Johann-K. & $\begin{array}{r}\text { (Zeitschrift-fur- } \\
\text { Nationalokono } \\
\text { mie); } 67(2), \\
1998 \text {, pages } \\
167-80 .\end{array}$ & & & & & & & & & \\
\hline $\begin{array}{r}\text { The Gini-Index as a } \\
\text { Measure of the Goodness } \\
\text { of Prediction }\end{array}$ & Riese,-Martin & $\begin{array}{r}\text { Bulletin-of- } \\
\text { Economic- } \\
\text { Research; } \\
\text { 49(2), April } \\
\text { 1997, pages } \\
\text { 127-35. }\end{array}$ & 1997 & Riese & Martin & $\cup \operatorname{Lin} z$ & 0 & 0,5 & 1 & 0 & 0,12 \\
\hline $\begin{array}{r}\text { Die Entwicklung der } \\
\text { Sozialpolitik in } \\
\text { reprasentativen und in } \\
\text { direkten Demokratien: } \\
\text { Konigsweg oder } \\
\text { Sackgasse? ... }\end{array}$ & $\begin{array}{r}\text { Schneider,-- } \\
\text { Friedrich }\end{array}$ & $\begin{array}{r}\text { Schweizerisch } \\
\text { e-Zeitschrift- } \\
\text { fur- } \\
\text { Volkswirtschaft } \\
\text {-undStatistik; } \\
135(3), 1999 \\
\text { pp. 387-406. }\end{array}$ & 1999 & Schneider & Friedrich & $U \operatorname{Linz}$ & 0 & 1 & 1 & 0 & \\
\hline $\begin{array}{r}\text { Blessing or Curse? } \\
\text { Foreign and Underground } \\
\text { Demand for Euro Notes: } \\
\text { Discussion }\end{array}$ & $\begin{array}{r}\text { Schneider,- } \\
\text { Friedrich }\end{array}$ & $\begin{array}{r}\text { Economic- } \\
\text { Policy:-A- } \\
\text { European- } \\
\text { Forum; 0(26), } \\
\text { April 1998, } \\
\text { pages 291-94. }\end{array}$ & 1998 & Schneider & Friedrich & $U \operatorname{Lin} z$ & 0 & 0,5 & 1 & 0 & \\
\hline $\begin{array}{l}\text { Tragic Choices and } \\
\text { ollective Decision-Making: } \\
\text { n Empirical Study of Voter } \\
\text { دreferences for Alternative } \\
\text {;ollective Decision-Making }\end{array}$ & $\begin{array}{r}\text { Pommerehne,- } \\
\text { W.-W.; Hart,-A.; } \\
\text { Schneider,-F. }\end{array}$ & $\begin{array}{r}\text { Economic- } \\
\text { Journal; } \\
107(442), \text { May } \\
1997, \text { pages } \\
618-35 .\end{array}$ & 1997 & Schneider & Friedrich & U Linz & 1,21 & 1 & 3 & 0,605 & \\
\hline $\begin{array}{r}\text { Jan the Shadow Economy } \\
\text { 3e Reduced through Major } \\
\text { Tax Reforms? An } \\
\text { Empirical Investigation for } \\
\text { Austria }\end{array}$ & $\begin{array}{r}\text { Schneider,- } \\
\text { Friedrich }\end{array}$ & $\begin{array}{r}\text { Public- } \\
\text { Finance; } \\
49(0) \text {, Suppl. } \\
\text { 1994, pages } \\
137-52 .\end{array}$ & 1994 & Schneider & Friedrich & U Linz & 0 & 1 & 1 & 0 & \\
\hline $\begin{array}{r}\text {; There a European Public } \\
\text { Choice Perspective? }\end{array}$ & $\begin{array}{r}\text { Schneider,- } \\
\text { Friedrich }\end{array}$ & $\begin{array}{r}\text { Kyklos; 48(2), } \\
\text { 1995, pages } \\
289-96 .\end{array}$ & 1995 & Schneider & Friedrich & U Linz & 0,37 & 0,5 & 1 & 0,185 & \\
\hline $\begin{array}{r}\text { Efficiency and } \\
\text { Effectiveness Control }\end{array}$ & $\begin{array}{r}\text { Bartel,-Rainer; } \\
\text { Schneider,- }\end{array}$ & $\begin{array}{l}\text { European- } \\
\text { Journal-of- }\end{array}$ & 1994 & Schneider & Friedrich & U Linz & 0 & 1 & 2 & 0 & \\
\hline
\end{tabular}




\begin{tabular}{|c|c|c|c|c|c|c|c|c|c|c|c|}
\hline $\begin{array}{r}\text { Based on Economic } \\
\text { Analysis: The Example of } \\
\text { the Austrian Court of Audit }\end{array}$ & Friedrich & $\begin{array}{r}\text { Law-and- } \\
\text { Economics; } \\
1(4), \\
\text { December } \\
\text { 1994, pages } \\
237-62 .\end{array}$ & & & & & & & & & \\
\hline $\begin{array}{l}\text { Deficits, Bailout and Free } \\
\text { Riders: Fiscal Elements of } \\
\text { a European Constitution }\end{array}$ & $\begin{array}{r}\text { Horstmann,- } \\
\text { Winfried; } \\
\text { Schneider,- } \\
\text { Friedrich }\end{array}$ & $\begin{array}{r}\text { Kyklos; } 47(3) \\
\text { 1994, pages } \\
\text { 355-83. }\end{array}$ & 1994 & Schneider & Friedrich & U Linz & 0,37 & 1 & 2 & 0,2466 & \\
\hline $\begin{array}{l}\text { The Relationship between } \\
\text { Efficiency and Profitability } \\
\text { with Respect to the Size of } \\
\text { Firms: An Empirical } \\
\text { Investigation for Austria }\end{array}$ & $\begin{array}{r}\text { Schneider,- } \\
\text { Friedrich }\end{array}$ & $\begin{array}{r}\text { Empirica; } \\
20(3), 1993, \\
\text { pages } 245-64 .\end{array}$ & 1993 & Schneider & Friedrich & U Linz & 0 & 1 & 1 & 0 & \\
\hline $\begin{array}{l}\text { The Next Twenty-Five } \\
\text { Years of Public Choice }\end{array}$ & $\begin{array}{l}\text { Rowley,-C.-K.; } \\
\text { Schneider,-F.; } \\
\text { Tollison,-R.-D. }\end{array}$ & $\begin{array}{r}\text { Public-Choice; } \\
77(1) \\
\text { September } \\
\text { 1993, pages } \\
1-7 .\end{array}$ & 1993 & Schneider & Friedrich & U Linz & 0,43 & 0,5 & 3 & 0,1075 & \\
\hline $\begin{array}{r}\text { An Inverse Relationship } \\
\text { between Efficiency and } \\
\text { Profitability According to } \\
\text { Size of (Upper-)Austrian } \\
\text { Firms? ... }\end{array}$ & $\begin{array}{r}\text { Schneider,- } \\
\text { Friedrich; } \\
\text { Lenzelbauer,- } \\
\text { Werner }\end{array}$ & $\begin{array}{r}\text { Small- } \\
\text { Business- } \\
\text { Economics; } \\
5(1) \text {, March } \\
\text { 1993, pages } \\
1-22 .\end{array}$ & 1993 & Schneider & Friedrich & U Linz & 0,35 & 1 & 2 & 0,2333 & \\
\hline $\begin{array}{r}\text { A European Public Choice } \\
\text { Perspective }\end{array}$ & $\begin{array}{r}\text { Schneider,- } \\
\text { Friedrich }\end{array}$ & $\begin{array}{r}\text { Public-Choice; } \\
71(3), \\
\text { September } \\
1991, \text { pages } \\
197-200 . \\
\end{array}$ & 1991 & Schneider & Friedrich & U Linz & 0,43 & 0,5 & 1 & 0,215 & \\
\hline $\begin{array}{l}\text { The "Mess" of the Public } \\
\text { Industrial Production in } \\
\text { Austria: A Typical Case of } \\
\text { Public Sector Inefficiency? }\end{array}$ & $\begin{array}{r}\text { Bartel,--Rainer; } \\
\text { Schneider,- } \\
\text { Friedrich }\end{array}$ & $\begin{array}{r}\text { Public-Choice; } \\
68(1-3) \\
\text { January } 1991 \\
\text { pages } 17-40 .\end{array}$ & 1991 & Schneider & Friedrich & U Linz & 0,43 & 1 & 2 & 0,2866 & $\begin{array}{r}\mathrm{F} \text {. } \\
\text { Schneider: }\end{array}$ \\
\hline $\begin{array}{r}\text { Privatization in Eastern } \\
\text { Europe and the Soviet } \\
\text { Union: Discussion }\end{array}$ & $\begin{array}{r}\text { Schneider,- } \\
\text { Friedrich }\end{array}$ & $\begin{array}{r}\text { Jahrbuch-der- } \\
\text { Wirtschaft- } \\
\text { Osteuropas; } \\
14(2), 1990, \\
\text { pages } 164-66 .\end{array}$ & 1990 & Schneider & Friedrich & U Linz & 0 & 0,5 & 1 & 0 & $\begin{array}{r}1,8791666 \\
6\end{array}$ \\
\hline
\end{tabular}




\begin{tabular}{|c|c|c|c|c|c|c|c|c|c|c|c|}
\hline $\begin{array}{r}\text { Lohnhohe und } \\
\text { Beschaftigung. Kaufkraft- } \\
\text { nd Kostenargument. (With } \\
\text { English summary.) }\end{array}$ & $\begin{array}{r}\text { Schuster,- } \\
\text { Helmut; Weiss,- } \\
\text { Christoph }\end{array}$ & 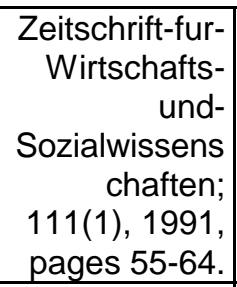 & 1991 & Schuster & Helmut & $U \operatorname{Lin} z$ & 0 & 0,5 & 2 & 0 & $\begin{array}{r}\mathrm{H} . \\
\text { Schuster: } 0\end{array}$ \\
\hline $\begin{array}{l}\text { Do Immigrants Displace } \\
\text { Young Native Workers: } \\
\text { The Austrian Experience }\end{array}$ & $\begin{array}{r}\text { Winter-Ebmer,- } \\
\text { Rudolf; } \\
\text { Zweimuller,- } \\
\text { Josef }\end{array}$ & $\begin{array}{r}\text { Journal-of- } \\
\text { Population- } \\
\text { Economics; } \\
\text { 12(2), May } \\
\text { 1999, pages } \\
327-40 . \\
\end{array}$ & 1999 & $\begin{array}{l}\text { Winter- } \\
\text { Ebmer }\end{array}$ & Rudolf & $U \operatorname{Lin} z$ & 0,44 & 1 & 2 & 0,2933 & \\
\hline $\begin{array}{l}\text { Lower and Upper Bounds } \\
\text { f Returns to Schooling: An } \\
\text { Exercise in IV Estimation } \\
\text { with Different Instruments }\end{array}$ & $\begin{array}{r}\text { Ichino,-Andrea; } \\
\text { Winter-Ebmer,- } \\
\text { Rudolf }\end{array}$ & $\begin{array}{r}\text { European- } \\
\text { Economic- } \\
\text { Review; } 43(4- \\
6), \text { April 1999, } \\
\text { pages 889- } \\
901 . \\
\end{array}$ & 1999 & $\begin{array}{r}\text { Winter- } \\
\text { Ebmer }\end{array}$ & Rudolf & $U \operatorname{Lin} z$ & 0,84 & 0,5 & 2 & 0,28 & \\
\hline $\begin{array}{r}\text { Firm-Size Wage } \\
\text { )ifferentials in Switzerland: } \\
\text { Evidence from Job- } \\
\text { Changers }\end{array}$ & $\begin{array}{r}\text { Winter-Ebmer,- } \\
\text { Rudolf; } \\
\text { Zweimuller,- } \\
\text { Josef }\end{array}$ & $\begin{array}{r}\text { American- } \\
\text { Economic- } \\
\text { Review; 89(2), } \\
\text { May 1999, } \\
\text { pages 89-93. } \\
\end{array}$ & 1999 & $\begin{array}{l}\text { Winter- } \\
\text { Ebmer }\end{array}$ & Rudolf & $U \operatorname{Lin} z$ & 1,74 & 0,5 & 2 & 0,58 & \\
\hline $\begin{array}{r}\text { Gender and Promotion in } \\
\text { the Academic Labour } \\
\text { Market }\end{array}$ & $\begin{array}{r}\text { van-der-Burg,-- } \\
\text { B.; Siegers,-J.; } \\
\text { Winter-Ebmer,-- } \\
\text { R. }\end{array}$ & $\begin{array}{r}\text { Labour; } 12(4) \\
\text { Winter 1998, } \\
\text { pages } 701-13 .\end{array}$ & 1998 & $\begin{array}{r}\text { Winter- } \\
\text { Ebmer }\end{array}$ & Rudolf & $U \operatorname{Lin} z$ & 0 & 1 & 3 & 0 & \\
\hline $\begin{array}{r}\text { Unknown Wage Offer } \\
\text { istribution and Job Search } \\
\text { Duration }\end{array}$ & $\begin{array}{r}\text { Winter-Ebmer,- } \\
\text { Rudolf }\end{array}$ & $\begin{array}{r}\text { Economics- } \\
\text { Letters; } 60(2), \\
\text { August } 1998, \\
\text { pages } 237-42 .\end{array}$ & 1998 & $\begin{array}{l}\text { Winter- } \\
\text { Ebmer }\end{array}$ & Rudolf & $U \operatorname{Lin} z$ & 0,26 & 0,5 & 1 & 0,13 & \\
\hline $\begin{array}{r}\text { Potential Unemployment } \\
\text { Benefit Duration and Spell } \\
\text { Length: Lessons from a } \\
\text { Quasi-Experiment in } \\
\text { Austria }\end{array}$ & $\begin{array}{r}\text { Winter-Ebmer, } \\
\text { Rudolf }\end{array}$ & $\begin{array}{r}\text { Oxford- } \\
\text { Bulletin-of- } \\
\text { Economics- } \\
\text { and-Statistics; } \\
60(1), \\
\text { February } \\
1998, \text { pages } \\
33-45 .\end{array}$ & 1998 & $\begin{array}{l}\text { Winter- } \\
\text { Ebmer }\end{array}$ & Rudolf & $U \operatorname{Lin} z$ & 0,76 & 1 & 1 & 0,76 & \\
\hline
\end{tabular}




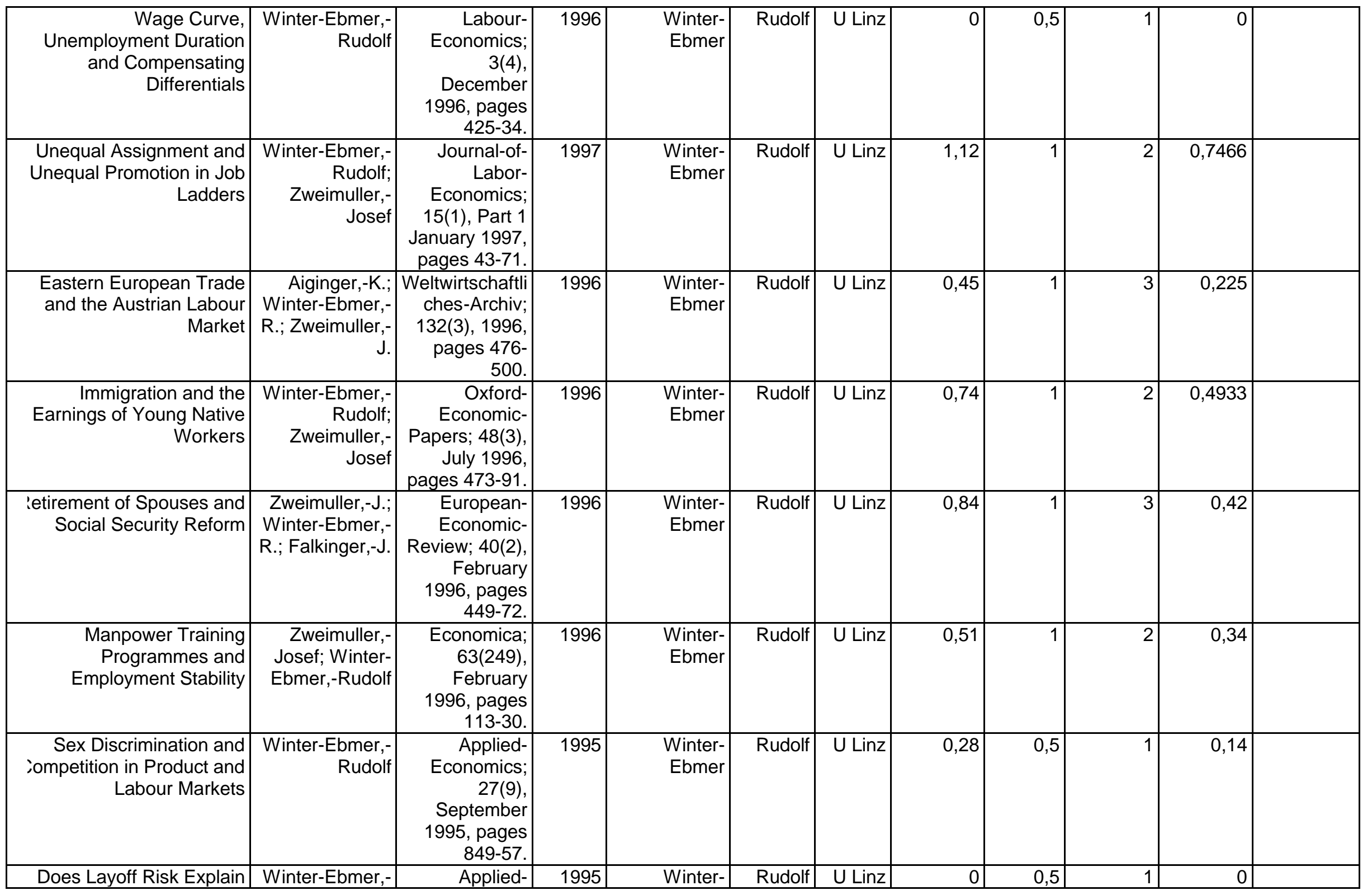




\begin{tabular}{|c|c|c|c|c|c|c|c|c|c|c|c|}
\hline $\begin{array}{r}\text { the Firm-Size Wage } \\
\text { Differential? }\end{array}$ & Rudolf & $\begin{array}{r}\text { Economics- } \\
\text { Letters; } 2(7), \\
\text { July } 1995, \\
\text { pages } 211-14 .\end{array}$ & & Ebmer & & & & & & & \\
\hline $\begin{array}{l}\text { nternal Labor Markets and } \\
\text { irm-Specific Determination } \\
\text { Earnings in the Presence } \\
\text { of Immigrant Workers }\end{array}$ & $\begin{array}{r}\text { Zweimuller,- } \\
\text { Josef; Winter- } \\
\text { Ebmer,-Rudolf }\end{array}$ & $\begin{array}{r}\text { Economics- } \\
\text { Letters; } 48(2) \\
\text { May } 1995, \\
\text { pages } 185-91 .\end{array}$ & 1995 & $\begin{array}{l}\text { Winter- } \\
\text { Ebmer }\end{array}$ & Rudolf & $U \operatorname{Linz}$ & 0,26 & 0,5 & 2 & 0,0866 & \\
\hline $\begin{array}{r}\text { lotivation for Migration and } \\
\text { Economic Success }\end{array}$ & $\begin{array}{r}\text { Winter-Ebmer, } \\
\text { Rudolf }\end{array}$ & $\begin{array}{r}\text { Journal-of- } \\
\text { Economic- } \\
\text { Psychology; } \\
15(2) \text {, June } \\
\text { 1994, pages } \\
269-84 .\end{array}$ & 1994 & $\begin{array}{l}\text { Winter- } \\
\text { Ebmer }\end{array}$ & Rudolf & $U \operatorname{Linz}$ & 0,24 & 1 & 1 & 0,24 & \\
\hline $\begin{array}{r}\text { Endogenous Growth, } \\
\text { Human Capital, and } \\
\text { Industry Wages }\end{array}$ & $\begin{array}{r}\text { Winter-Ebmer, } \\
\text { Rudolf }\end{array}$ & $\begin{array}{r}\text { Bulletin-of- } \\
\text { Economic- } \\
\text { Research; } \\
\text { 46(4), October } \\
\text { 1994, pages } \\
289-314 . \\
\end{array}$ & 1994 & $\begin{array}{r}\text { Winter- } \\
\text { Ebmer }\end{array}$ & Rudolf & $U \operatorname{Linz}$ & 0 & 1 & 1 & 0 & \\
\hline $\begin{array}{l}\text { Occupational Segregation } \\
\text { and Career Advancement }\end{array}$ & $\begin{array}{r}\text { Winter-Ebmer,- } \\
\text { Rudolf; } \\
\text { Zweimuller,- } \\
\text { Josef }\end{array}$ & $\begin{array}{r}\text { Economics- } \\
\text { Letters; 39(2), } \\
\text { June 1992, } \\
\text { pages 229-34. }\end{array}$ & 1992 & $\begin{array}{l}\text { Winter- } \\
\text { Ebmer }\end{array}$ & Rudolf & $U \operatorname{Linz}$ & 0,26 & 0,5 & 2 & 0,0866 & \\
\hline $\begin{array}{r}\text { Do They Come Back } \\
\text { Again? Job Search, } \\
\text { Labour Market } \\
\text { Segmentation and State } \\
\text { Dependence as } \\
\text { Explanations of Repeat } \\
\text { Unemployment }\end{array}$ & $\begin{array}{r}\text { Winter-Ebmer,- } \\
\text { Rudolf; } \\
\text { Zweimuller,- } \\
\text { Josef }\end{array}$ & $\begin{array}{r}\text { Empirical- } \\
\text { Economics; } \\
17(2), 1992, \\
\text { pages } 273-92 .\end{array}$ & 1992 & $\begin{array}{l}\text { Winter- } \\
\text { Ebmer }\end{array}$ & Rudolf & $U \operatorname{Linz}$ & 0 & 1 & 2 & 0 & \\
\hline $\begin{array}{c}\text { Some Micro Evidence on } \\
\text { Inemployment Persistence }\end{array}$ & $\begin{array}{r}\text { Winter-Ebmer,- } \\
\text { Rudolf }\end{array}$ & $\begin{array}{r}\text { Oxford- } \\
\text { Bulletin-of- } \\
\text { Economics- } \\
\text { and-Statistics; } \\
53(1) \\
\text { February } \\
1991, \text { pages } \\
27-43 . \\
\end{array}$ & 1991 & $\begin{array}{l}\text { Winter- } \\
\text { Ebmer }\end{array}$ & Rudolf & U Linz & 0,76 & 1 & 1 & 0,76 & $\begin{array}{l}\text { R. Winter- } \\
\text { Ebmer: }\end{array}$ \\
\hline Die Beveridge-Surve: Ein & Winter-Ebmer,-- & Konjunkturpolit & 1991 & Winter- & Rudolf & $U \operatorname{Linz}$ & 0 & 1 & 1 & 0 & 5,5816666 \\
\hline
\end{tabular}




\begin{tabular}{|r|r|r|r|r|r|r|r|}
\hline $\begin{array}{r}\text { altes Konzept neu } \\
\text { Interpretiert. (The } \\
\text { Beveridge-Curve: New } \\
\text { Interpretations of an Old } \\
\text { Concept.) }\end{array}$ & Von-Rudolf $\begin{array}{r}\text { ik; 37(1-2), } \\
\text { January 1991, } \\
\text { pages 45-65. }\end{array}$ & & Ebmer & & & & \\
\hline
\end{tabular}


London School of Economics

\begin{tabular}{|c|c|c|c|c|c|c|c|c|c|c|c|}
\hline & Author(s) & Journal & Year & & & & $\begin{array}{l}\text { Impact } \\
\text { Factor }\end{array}$ & $\begin{array}{c}\text { Seiten- } \\
\text { korr. }\end{array}$ & Autoren & Impact & personal Impact \\
\hline $\begin{array}{l}\text { Sales Taxes and Prices: An } \\
\text { Empirical Analysis }\end{array}$ & $\begin{array}{l}\text { Besley,-Timothy-J.; } \\
\text { Rosen,-Harvey-S. }\end{array}$ & $\begin{array}{l}\text { National-Tax-Journal; 52(2), } \\
\text { June 1999, pp. 157-78. }\end{array}$ & 1999 & Besley & Timothy J. & LSE & 0.75 & 1 & 2 & 0.5 & \\
\hline $\begin{array}{l}\text { Vertical Externalities in Tax Setting: } \\
\text { Evidence from Gasoline and } \\
\text { Cigarettes }\end{array}$ & $\begin{array}{l}\text { Besley,-Timothy-J.; } \\
\text { Rosen,-Harvey-S. }\end{array}$ & $\begin{array}{l}\text { Journal-of-Public-Economics; } \\
\text { 70(3), December 1998, } \\
\text { pp. 383-98. }\end{array}$ & 1998 & Besley & Timothy J. & $\overline{\mathrm{LSE}}$ & 0.82 & 1 & 2 & 0.546666667 & $\begin{array}{l}\text { T. J. Besley: } \\
1.046666667\end{array}$ \\
\hline Value-Added Tax Options for India & $\begin{array}{l}\text { Burgess,-R.; } \\
\text { Howes,-S.; } \\
\text { Stern,-N. }\end{array}$ & $\begin{array}{l}\text { International-Tax-and-Public- } \\
\text { Finance; } 2(1), \text { May } 1995, \\
\text { pp. 109-41. }\end{array}$ & 1995 & Burgess & Robin & LSE & 0 & 1 & 3 & 0 & \\
\hline $\begin{array}{l}\text { The Behaviour of State Firms in } \\
\text { Eastern Europe, Pre-privatisation }\end{array}$ & $\begin{array}{l}\text { Aghion,-P.; } \\
\text { Blanchard,-O.-J.; } \\
\text { Burgess,-R. }\end{array}$ & $\begin{array}{l}\text { European-Economic-Review; } \\
38(6) \text {, June 1994, pp. } 1327- \\
49 .\end{array}$ & 1994 & Burgess & Robin & LSE & 0.84 & 1 & 3 & 0.42 & \\
\hline Taxation and Development & $\begin{array}{l}\text { Burgess,-Robin; } \\
\text { Stern,-Nicholas }\end{array}$ & $\begin{array}{l}\text { Journal-of-Economic- } \\
\text { Literature; 31(2), June 1993, } \\
\text { pp. } 762-830 .\end{array}$ & 1993 & Burgess & Robin & LSE & 3.78 & 1 & 2 & 2.52 & R. Burgess: 2.94 \\
\hline $\begin{array}{l}\text { Consistent Hypothesis Testing in } \\
\text { Semiparametric and Nonparametric } \\
\text { Models for Econometric Time Series }\end{array}$ & $\begin{array}{l}\text { Chen,-Xiaohong; } \\
\text { Fan,-Yanqin }\end{array}$ & $\begin{array}{l}\text { Journal-of-Econometrics; } \\
91(2), \text { August 1999, pp. } 373- \\
401 .\end{array}$ & 1999 & Chen & Xiaohong & $\overline{\mathrm{LSE}}$ & 1.06 & 1 & 2 & 0.706666667 & \\
\hline $\begin{array}{l}\text { Nonparametric Adaptive Learning } \\
\text { with Feedback }\end{array}$ & $\begin{array}{l}\text { Chen,-Xiaohong; } \\
\text { White,-Halbert }\end{array}$ & $\begin{array}{l}\text { Journal-of-Economic-Theory; } \\
\text { 82(1), September 1998, } \\
\text { pp. 190-222. }\end{array}$ & 1998 & Chen & Xiaohong & LSE & 0.93 & 1 & 2 & 0.62 & \\
\hline $\begin{array}{l}\text { Central Limit and Functional Central } \\
\text { Limit Theorems for Hilbert-Valued } \\
\text { Dependent Heterogeneous Arrays } \\
\text { with Applications }\end{array}$ & $\begin{array}{l}\text { Chen,-Xiaohong; } \\
\text { White,-Halbert }\end{array}$ & $\begin{array}{l}\text { Econometric-Theory; 14(2), } \\
\text { April 1998, pp. 260-84. }\end{array}$ & 1998 & Chen & Xiaohong & LSE & 0.5 & 1 & 2 & 0.333333333 & \\
\hline $\begin{array}{l}\text { Sieve Extremum Estimates for } \\
\text { Weakly Dependent Data }\end{array}$ & $\begin{array}{l}\text { Chen,-Xiaohong; } \\
\text { Shen,-Xiaotong }\end{array}$ & $\begin{array}{l}\text { Econometrica; 66(2), March } \\
\text { 1998, pp. 289-314. }\end{array}$ & 1998 & Chen & Xiaohong & $\overline{L S E}$ & 2.22 & 1 & 2 & 1.48 & \\
\hline $\begin{array}{l}\text { Laws of Large Numbers for Hilbert } \\
\text { Space-Valued Mixingales with } \\
\text { Applications }\end{array}$ & $\begin{array}{l}\text { Chen,-Xiaohong; } \\
\text { White,-Halbert }\end{array}$ & $\begin{array}{l}\text { Econometric-Theory; } 12(2), \\
\text { June 1996, pp. 284-304. }\end{array}$ & 1996 & Chen & Xiaohong & $\overline{L S E}$ & 0.5 & 1 & 2 & 0.333333333 & $\begin{array}{l}\text { X. Chen: } \\
\text { 3.473333333 }\end{array}$ \\
\hline $\begin{array}{l}\text { Poverty Measurement with } \\
\text { Contaminated Data: A Robust } \\
\text { Approach }\end{array}$ & $\begin{array}{l}\text { Cowell,-Frank-A.; } \\
\text { Victoria-Feser,-- } \\
\text { Maria-Pia } \\
\end{array}$ & $\begin{array}{l}\text { European-Economic-Review; } \\
\text { 40(9), December 1996, } \\
\text { pp. 1761-71. }\end{array}$ & 1996 & Cowell & Frank A. & LSE & 0.84 & 1 & 2 & 0.56 & \\
\hline $\begin{array}{l}\text { Robustness Properties of Inequality } \\
\text { Measures }\end{array}$ & $\begin{array}{l}\text { Cowell,-Frank-A.; } \\
\text { Victoria-Feser,-- } \\
\text { Maria-Pia }\end{array}$ & $\begin{array}{l}\text { Econometrica; 64(1), January } \\
\text { 1996, pp. } 77-101 .\end{array}$ & 1996 & Cowell & Frank A. & LSE & 2.22 & 1 & 2 & 1.48 & \\
\hline $\begin{array}{l}\text { How Much Inequality Can We } \\
\text { Explain? A Methodology and an } \\
\text { Application to the United States }\end{array}$ & $\begin{array}{l}\text { Cowell,-Frank-A.; } \\
\text { Jenkins,-Stephen-P. }\end{array}$ & $\begin{array}{l}\text { Economic-Journal; 105(429), } \\
\text { March 1995, pp. 421-30. }\end{array}$ & 1995 & Cowell & Frank A. & LSE & 1.21 & 0.5 & 2 & 0.403333333 & \\
\hline $\begin{array}{l}\text { Monotonicity, Dominance and the } \\
\text { Pareto Principle }\end{array}$ & \begin{tabular}{|l|} 
Amiel,-Yoram; \\
Cowell,-Frank-A.
\end{tabular} & $\begin{array}{l}\text { Economics-Letters; 45(4), } \\
\text { August 1994, pp. 447-50. }\end{array}$ & 1994 & Cowell & Frank A. & LSE & 0.26 & 0.5 & 2 & 0.086666667 & \\
\hline $\begin{array}{l}\text { Parametric Equivalence Scales and } \\
\text { Scale Relativities }\end{array}$ & $\begin{array}{l}\text { Jenkins,-Stephen-P.; } \\
\text { Cowell,-Frank-A. }\end{array}$ & $\begin{array}{l}\text { Economic-Journal; 104(425), } \\
\text { July 1994, pp. 891-900. }\end{array}$ & 1994 & Cowell & Frank A. & LSE & 1.21 & 0.5 & 2 & 0.403333333 & \\
\hline $\begin{array}{l}\text { Errata [Dwarfs and Giants in the } \\
\text { 1980s: Trends in the UK Income } \\
\text { Distribution]. }\end{array}$ & $\begin{array}{l}\text { Jenkins,-Stephen-P.; } \\
\text { Cowell,-Frank-A. }\end{array}$ & $\begin{array}{l}\text { Fiscal-Studies; 15(2), May } \\
\text { 1994, pp. } 105 .\end{array}$ & 1994 & Cowell & Frank A. & LSE & 0 & 1 & 2 & 0 & \\
\hline
\end{tabular}


London School of Economics

\begin{tabular}{|c|c|c|c|c|c|c|c|c|c|c|c|}
\hline & Author(s) & Journal & Year & & & & \begin{tabular}{|l|} 
Impact \\
Factor
\end{tabular} & $\begin{array}{c}\text { Seiten- } \\
\text { korr. }\end{array}$ & Autoren & Impact & personal Impact \\
\hline $\begin{array}{l}\text { Dwarfs and Giants in the 1980s: } \\
\text { Trends in the UK Income } \\
\text { Distribution }\end{array}$ & \begin{tabular}{|l|} 
Jenkins,-Stephen-P.; \\
Cowell,-Frank-A.
\end{tabular} & $\begin{array}{l}\text { Fiscal-Studies; 15(1), } \\
\text { February 1994, pp. 99-118. }\end{array}$ & 1994 & Cowell & Frank A. & LSE & 0 & 1 & 2 & 0 & \\
\hline $\begin{array}{l}\text { Equivalence Scale Relativities and } \\
\text { the Extent of Inequality and Poverty }\end{array}$ & $\begin{array}{l}\text { Coulter,-F.-A.-E.; } \\
\text { Cowell,-F.-A.; } \\
\text { Jenkins,-S.-P. }\end{array}$ & \begin{tabular}{|l} 
Economic-Journal; 102(414), \\
September 1992, pp. 1067-82.
\end{tabular} & 1992 & Cowell & Frank A. & LSE & 1.21 & 1 & 3 & 0.605 & \\
\hline $\begin{array}{l}\text { Measurement of Income Inequality: } \\
\text { Experimental Test by Questionnaire }\end{array}$ & $\begin{array}{l}\text { Amiel,-Yoram; } \\
\text { Cowell,-Frank-A. }\end{array}$ & $\begin{array}{l}\text { Journal-of-Public-Economics; } \\
47(1) \text {, February 1992, pp. 3- } \\
26 .\end{array}$ & 1992 & Cowell & Frank A. & LSE & 0.82 & 1 & 2 & 0.546666667 & \\
\hline $\begin{array}{l}\text { Differences in Needs and } \\
\text { Assessment of Income Distributions }\end{array}$ & $\begin{array}{l}\text { Coulter,-F.-A.-E.; } \\
\text { Cowell,-F.-A.; } \\
\text { Jenkins,-S.-P. }\end{array}$ & $\begin{array}{l}\text { Bulletin-of-Economic- } \\
\text { Research; 44(2), April 1992, } \\
\text { pp. 77-124. }\end{array}$ & 1992 & Cowell & Frank A. & LSE & 0 & 1 & 3 & 0 & \\
\hline $\begin{array}{l}\text { Grouping Bounds for Inequality } \\
\text { Measures under Alternative } \\
\text { Informational Assumptions }\end{array}$ & Cowell,-Frank-A. & $\begin{array}{l}\text { Journal-of-Econometrics; } \\
48(1-2), \text { April-May 1991, pp. 1- } \\
\text { 14. }\end{array}$ & 1991 & Cowell & Frank A. & LSE & 1.06 & 1 & 1 & 1.06 & F. A. Cowell: 5.145 \\
\hline $\begin{array}{l}\text { Hierarchical Bayes Estimation of } \\
\text { Unemployment Rates for the States } \\
\text { of the U.S. }\end{array}$ & Datta,-G.-S., et-al. & $\begin{array}{l}\text { Journal-of-the-American- } \\
\text { Statistical-Association; } \\
\text { 94(448), December 1999, } \\
\text { pp. 1074-82. }\end{array}$ & 1999 & Datta & G. S. & LSE & 1.58 & 0.5 & 3 & 0.395 & G. S. Datta: 0,395 \\
\hline Wealth, Enterprise and Credit Policy & $\begin{array}{l}\text { de-Meza,-David; } \\
\text { Webb,-David }\end{array}$ & $\begin{array}{l}\text { Economic-Journal; 109(455), } \\
\text { April 1999, pp. 153-63. }\end{array}$ & 1999 & De Meza & David & LSE & 1.21 & 1 & 2 & 0.806666667 & \\
\hline $\begin{array}{l}\text { Too Much Monitoring, Not Enough } \\
\text { Performance Pay }\end{array}$ & $\begin{array}{l}\text { de-Meza,-David; } \\
\text { Southey,-Clive }\end{array}$ & $\begin{array}{l}\text { Economic-Journal; 109(454), } \\
\text { March 1999, pp. C126-39. }\end{array}$ & 1999 & De Meza & David & LSE & 1.21 & 1 & 2 & 0.806666667 & \\
\hline $\begin{array}{l}\text { Does Asset Ownership Always } \\
\text { Motivate Managers? Outside } \\
\text { Options and the Property Rights } \\
\text { Theory of the Firm }\end{array}$ & $\begin{array}{l}\text { de-Meza,-David; } \\
\text { Lockwood,-Ben }\end{array}$ & $\begin{array}{l}\text { Quarterly-Journal-of- } \\
\text { Economics; 113(2), May 1998, } \\
\text { pp. 361-86. }\end{array}$ & 1998 & De Meza & David & LSE & 3.2 & 1 & 2 & 2.133333333 & \\
\hline $\begin{array}{l}\text { Everyone May Benefit from } \\
\text { Subsidising Entry to Risky } \\
\text { Occupations }\end{array}$ & $\begin{array}{l}\text { Black,-Jane; de- } \\
\text { Meza,-David }\end{array}$ & $\begin{array}{l}\text { Journal-of-Public-Economics; } \\
\text { 66(3), December 1997, } \\
\text { pp. 409-24. }\end{array}$ & 1997 & De Meza & David & LSE & 0.82 & 1 & 2 & 0.5466666667 & \\
\hline $\begin{array}{l}\text { In Praise of Inequality: Public Good } \\
\text { Provision and Income Distribution }\end{array}$ & \begin{tabular}{|l|} 
Itaya,-Jun-ichi; de- \\
Meza,-David; Myles,- \\
Gareth-D. \\
\end{tabular} & $\begin{array}{l}\text { Economics-Letters; 57(3), } \\
\text { December 1997, pp. 289-96. } \\
\end{array}$ & 1997 & De Meza & David & LSE & 0.26 & 0.5 & 3 & 0.065 & \\
\hline $\begin{array}{l}\text { Product Diversity under Monopoly: } \\
\text { Two High Quality Results }\end{array}$ & de-Meza,-David & $\begin{array}{l}\text { Bulletin-of-Economic- } \\
\text { Research; 49(2), April 1997, } \\
\text { pp. 169-71. }\end{array}$ & 1997 & De Meza & David & LSE & 0 & 0.5 & 1 & 0 & \\
\hline $\begin{array}{l}\text { A Generalized Equivalence Property } \\
\text { of Mixed International VAT Regimes }\end{array}$ & Genser,-Bernd & $\begin{array}{l}\text { Scandinavian-Journal-of- } \\
\text { Economics; 98(2), June 1996, } \\
\text { pp. 253-62. }\end{array}$ & 1996 & De Meza & David & LSE & 0.48 & 0.5 & 1 & 0.24 & \\
\hline $\begin{array}{l}\text { The Borrower's Curse: Optimism, } \\
\text { Finance and Entrepreneurship }\end{array}$ & $\begin{array}{l}\text { de-Meza,-David; } \\
\text { Southey,-Clive }\end{array}$ & $\begin{array}{l}\text { Economic-Journal; 106(435), } \\
\text { March 1996, pp. 375-86. }\end{array}$ & 1996 & De Meza & David & LSE & 1.21 & 1 & 2 & 0.806666667 & \\
\hline $\begin{array}{l}\text { House Price, the Supply of } \\
\text { Collateral and the Enterprise } \\
\text { Economy }\end{array}$ & $\begin{array}{l}\text { Black,-Jane; de- } \\
\text { Meza,-David; } \\
\text { Jeffreys,-David }\end{array}$ & $\begin{array}{l}\text { Economic-Journal; 106(434), } \\
\text { January 1996, pp. 60-75. }\end{array}$ & 1996 & De Meza & David & LSE & 1.21 & 1 & 3 & 0.605 & \\
\hline $\begin{array}{l}\text { When Are Origin and Destination } \\
\text { Regimes Equivalent? }\end{array}$ & $\begin{array}{l}\text { Lockwood,-B.; de- } \\
\text { Meza,-D.; Myles,-G.- } \\
\text { D. }\end{array}$ & $\begin{array}{l}\text { International-Tax-and-Public- } \\
\text { Finance; 1(1), May 1994, } \\
\text { pp. 5-24. }\end{array}$ & 1994 & De Meza & David & LSE & 0 & 1 & 3 & 0 & \\
\hline
\end{tabular}


London School of Economics

\begin{tabular}{|c|c|c|c|c|c|c|c|c|c|c|c|}
\hline & Author(s) & Journal & Year & & & & $\begin{array}{l}\text { Impact } \\
\text { Factor }\end{array}$ & \begin{tabular}{|c|} 
Seiten- \\
korr.
\end{tabular} & Autoren & Impact & personal Impact \\
\hline Price-Reducing Taxation & $\begin{array}{l}\text { de-Meza,-D.; } \\
\text { Maloney,-J.; Myles,- } \\
\text { G.-D. }\end{array}$ & $\begin{array}{l}\text { Economics-Letters; 47(1), } \\
\text { January 1995, pp. 77-81. }\end{array}$ & 1995 & De Meza & David & LSE & 0.26 & $\begin{array}{ll}0.5 \\
\end{array}$ & 3 & 0.065 & \\
\hline Unnatural Monopoly & $\begin{array}{l}\text { Estrin,-Saul; de- } \\
\text { Meza,-David }\end{array}$ & $\begin{array}{l}\text { Journal-of-Public-Economics; } \\
\text { 57(3), July 1995, pp. 471-88. }\end{array}$ & 1995 & De Meza & David & LSE & 0.82 & 1 & 2 & 0.546666667 & \\
\hline $\begin{array}{l}\text { On the European Union VAT } \\
\text { Proposals: The Superiority of Origin } \\
\text { over Destination Taxation }\end{array}$ & $\begin{array}{l}\text { Lockwood,-Ben; de- } \\
\text { Meza,-David; Myles,- } \\
\text { Gareth } \\
\end{array}$ & \begin{tabular}{|l} 
Fiscal-Studies; $16(1)$, \\
February 1995, pp. 1-17.
\end{tabular} & 1995 & De Meza & David & LSE & 0 & 1 & 3 & 0 & \\
\hline The Nature of Credit-Market Failure & $\begin{array}{l}\text { Black,-Jane; de- } \\
\text { Meza,-David }\end{array}$ & $\begin{array}{l}\text { Economics-Letters; 46(3), } \\
\text { November 1994, pp. 243-49. }\end{array}$ & 1994 & De Meza & David & LSE & 0.26 & 0.5 & 2 & 0.086666667 & \\
\hline $\begin{array}{l}\text { Creating a Good Atmosphere: } \\
\text { Minimum Participation for Tackling } \\
\text { the 'Greenhouse Effect.' }\end{array}$ & $\begin{array}{l}\text { Black,-Jane; Levi,-- } \\
\text { Maurice-D.; de-- } \\
\text { Meza,-David }\end{array}$ & $\begin{array}{l}\text { Economica; 60(239), August } \\
\text { 1993, pp. 281-93. }\end{array}$ & 1993 & De Meza & David & LSE & 0.51 & 1 & 3 & 0.255 & \\
\hline $\begin{array}{l}\text { Systematic Price Differences } \\
\text { between Successive Auctions Are } \\
\text { No Anomaly }\end{array}$ & $\begin{array}{l}\text { Black,-Jane; De- } \\
\text { Meza,-David }\end{array}$ & $\begin{array}{l}\text { Journal-of-Economics-and- } \\
\text { Management-Strategy; 1(4), } \\
\text { 1992, pp. 607-28. }\end{array}$ & 1992 & De Meza & David & LSE & 0 & 1 & 2 & 0 & \\
\hline Efficient Credit Rationing & $\begin{array}{l}\text { de-Meza,-David; } \\
\text { Webb,-David-C. }\end{array}$ & $\begin{array}{l}\text { European-Economic-Review; } \\
\text { 36(6), August 1992, pp. 1277- } \\
\text { 90. }\end{array}$ & 1992 & De Meza & David & LSE & 0.84 & 1 & 2 & 0.56 & \\
\hline $\begin{array}{l}\text { The Social Efficiency of Private } \\
\text { Decisions to Enforce Property } \\
\text { Rights }\end{array}$ & $\begin{array}{l}\text { de-Meza,-David; } \\
\text { Gould,-J.-R. }\end{array}$ & $\begin{array}{l}\text { Journal-of-Political-Economy; } \\
\text { 100(3), June 1992, pp. 561- } \\
80 .\end{array}$ & 1992 & De Meza & David & LSE & 1.95 & 1 & 2 & 1.3 & \\
\hline $\begin{array}{l}\text { A Strategic Motivation for } \\
\text { Commodity Bundling }\end{array}$ & $\begin{array}{l}\text { Carbajo,-J.; de- } \\
\text { Meza,-D.; } \\
\text { Seidmann,-D.-J. }\end{array}$ & $\begin{array}{l}\text { Journal-of-Industrial- } \\
\text { Economics; 38(3), March } \\
\text { 1990, pp. 283-98. }\end{array}$ & 1990 & De Meza & David & LSE & 0.6 & 1 & 3 & 0.3 & \\
\hline $\begin{array}{l}\text { Risk, Adverse Selection and Capital } \\
\text { Market Failure }\end{array}$ & $\begin{array}{l}\text { de-Meza,-David; } \\
\text { Webb,-David }\end{array}$ & $\begin{array}{l}\text { Economic-Journal; 100(399), } \\
\text { March 1990, pp. 206-14. }\end{array}$ & 1990 & De Meza & David & LSE & 1.21 & 0.5 & 2 & 0.403333333 & \begin{tabular}{|l|} 
D. De Meza: \\
9.526666667
\end{tabular} \\
\hline $\begin{array}{l}\text { Richard Goodwin: A Short } \\
\text { Appreciation }\end{array}$ & $\begin{array}{l}\text { Desai,-Meghnad; } \\
\text { Ormerod,-Paul }\end{array}$ & $\begin{array}{l}\text { Economic-Journal; 108(450), } \\
\text { September 1998, pp. 1431-35. }\end{array}$ & \begin{tabular}{|l}
1998 \\
\end{tabular} & Desai & Meghnad & LSE & 1.21 & 0.5 & 2 & 0.403333333 & \\
\hline $\begin{array}{l}\text { Debating the British Disease: The } \\
\text { Centrality of Profit }\end{array}$ & Desai,-Meghnad & $\begin{array}{l}\text { New-Political-Economy; 1(1), } \\
\text { March 1996, pp. 79-93. }\end{array}$ & 1996 & Desai & Meghnad & LSE & 0 & 1 & 1 & 0 & \\
\hline John Denis Sargan & $\begin{array}{l}\text { Desai,-M.-J.; } \\
\text { Hendry,-D.-F.; } \\
\text { Mizon,-G.-E. }\end{array}$ & $\begin{array}{l}\text { Economic-Journal; 107(443), } \\
\text { July 1997, pp. 1121-25. }\end{array}$ & 1997 & Desai & Meghnad & LSE & 1.21 & 0.5 & 3 & 0.3025 & \\
\hline Finding a New Role for the State & Desai,-Meghnad & $\begin{array}{l}\text { New-Economy; 4(1), Spring } \\
\text { 1997, pp. 22-25. }\end{array}$ & 1997 & Desai & Meghnad & LSE & 0 & 0.5 & 1 & 0 & \\
\hline $\begin{array}{l}\text { An Endogenous Growth Cycle with } \\
\text { Vintage Capital }\end{array}$ & Desai,-Meghnad & $\begin{array}{l}\text { Economics-of-Planning; } 28(2- \\
3), 1995 \text {, pp. 87-91. }\end{array}$ & 1995 & Desai & Meghnad & LSE & 0 & 0.5 & $\overline{1}$ & 0 & \\
\hline $\begin{array}{l}\text { Rice, Fish and Famine Relief: } \\
\text { Comment }\end{array}$ & Desai,-Meghnad & $\begin{array}{l}\text { European-Journal-of-Political- } \\
\text { Economy; 10(3), Oct. 1994, } \\
\text { pp. 607-10. }\end{array}$ & 1994 & Desai & Meghnad & LSE & 0 & 0.5 & 1 & 0 & \\
\hline $\begin{array}{l}\text { The Measurement Problem in } \\
\text { Economics }\end{array}$ & Desai,-Meghnad & $\begin{array}{l}\text { Scottish-Journal-of-Political- } \\
\text { Economy; 41(1), Feb. 1994, } \\
\text { pp. 34-42. }\end{array}$ & 1994 & Desai & Meghnad & LSE & 0.46 & 0.5 & 1 & 0.23 & \\
\hline $\begin{array}{l}\text { Is There Life after Mahalanobis? } \\
\text { The Political Economy of India's } \\
\text { New Economic Policy }\end{array}$ & Desai,-Meghnad & $\begin{array}{l}\text { Indian-Economic-Review; } \\
\text { 27(0), Special Issue 1992, } \\
\text { pp. 155-64. }\end{array}$ & 1992 & Desai & Meghnad & LSE & 0 & 0.5 & 1 & 0 & \\
\hline
\end{tabular}


London School of Economics

\begin{tabular}{|c|c|c|c|c|c|c|c|c|c|c|c|}
\hline & Author(s) & Journal & Year & & & & $\begin{array}{l}\text { Impact } \\
\text { Factor }\end{array}$ & $\begin{array}{c}\text { Seiten- } \\
\text { korr. }\end{array}$ & Autoren & Impact & personal Impact \\
\hline Population and Poverty in Africa & Desai,-Meghnad & $\begin{array}{l}\text { African-Development- } \\
\text { Review/Revue-africaine-de- } \\
\text { developpement; 4(2), Dec. } \\
\text { 1992, pp. 63-78. }\end{array}$ & 1992 & Desai & Meghnad & LSE & 0 & 1 & 1 & 0 & \\
\hline $\begin{array}{l}\text { The Agrarian Crisis in Medieval } \\
\text { England: A Malthusian Tragedy or } \\
\text { a Failure of Entitlements? }\end{array}$ & Desai,-Meghnad & $\begin{array}{l}\text { Bulletin-of-Economic- } \\
\text { Research; 43(3), July 1991, } \\
\text { pp. 223-58. }\end{array}$ & 1991 & Desai & Meghnad & LSE & 0 & 1 & 1 & 0 & \\
\hline $\begin{array}{l}\text { Human Development: Concepts } \\
\text { and Measurement }\end{array}$ & Desai,-Meghnad & $\begin{array}{l}\text { European-Economic-Review; } \\
\text { 35(2-3), April 1991, pp. 350- } \\
\text { 57. }\end{array}$ & 1991 & Desai & Meghnad & LSE & 0.84 & 0.5 & 1 & 0.42 & $\begin{array}{l}\text { M. Desai: } \\
\text { 1.355833333 }\end{array}$ \\
\hline $\begin{array}{l}\text { The Specification of Earnings } \\
\text { Functions: Tests and Implications }\end{array}$ & $\begin{array}{l}\text { Dougherty,-C.-R.-S.; } \\
\text { Jimenez,-E. }\end{array}$ & $\begin{array}{l}\text { Economics-of-Education- } \\
\text { Review; 10(2), 1991, pp. 85- } \\
98 .\end{array}$ & 1991 & Dougherty & Christopher & LSE & 0 & 1 & 2 & 0 & C. Dougherty: 0 \\
\hline $\begin{array}{l}\text { Optimal Debt Contracts and the } \\
\text { Single-Crossing Condition }\end{array}$ & $\begin{array}{l}\text { Faure-Grimaud,-- } \\
\text { Antoine; Mariotti,-- } \\
\text { Thomas }\end{array}$ & $\begin{array}{l}\text { Economics-Letters; 65(1), } \\
\text { October 1999, pp. 85-89. }\end{array}$ & 1999 & $\begin{array}{l}\text { Faure- } \\
\text { Grimaud }\end{array}$ & Antoine & LSE & 0.26 & 0.5 & 2 & 0.086666667 & \\
\hline $\begin{array}{l}\text { The Endogenous Transaction Costs } \\
\text { of Delegated Auditing }\end{array}$ & $\begin{array}{l}\text { Faure-Grimaud,-A.; } \\
\text { Laffont,-J.-J.; } \\
\text { Martimort,-D. }\end{array}$ & $\begin{array}{l}\text { European-Economic-Review; } \\
43(4-6) \text {, April 1999, pp. 1039- } \\
\text { 48. }\end{array}$ & 1999 & $\begin{array}{l}\text { Faure- } \\
\text { Grimaud }\end{array}$ & Antoine & LSE & 0.84 & 0.5 & 3 & 0.21 & \\
\hline $\begin{array}{l}\text { Structure financiere et concurrence } \\
\text { imparfaite: Modigliani-Miller } 40 \text { ans } \\
\text { apres. }\end{array}$ & \begin{tabular}{|l}
$\begin{array}{l}\text { Faure-Grimaud,-- } \\
\text { Antoine }\end{array}$ \\
\end{tabular} & $\begin{array}{l}\text { Revue-d'Economie-Politique; } \\
\text { 108(1), Jan.-Feb. 1998, } \\
\text { pp. 15-36. }\end{array}$ & 1998 & $\begin{array}{l}\text { Faure- } \\
\text { Grimaud }\end{array}$ & Antoine & LSE & 0 & 1 & 1 & 0 & \\
\hline The Regulation of Predatory Firms & $\begin{array}{l}\text { Faure-Grimaud,-- } \\
\text { Antoine }\end{array}$ & $\begin{array}{l}\text { Journal-of-Economics-and- } \\
\text { Management-Strategy; 6(4), } \\
\text { 1997, pp. 849-76. } \\
\end{array}$ & 1997 & $\begin{array}{l}\text { Faure- } \\
\text { Grimaud }\end{array}$ & Antoine & LSE & 0 & 1 & 1 & 0 & $\begin{array}{l}\text { A. Faure-Grimaud: } \\
0.296666667\end{array}$ \\
\hline $\begin{array}{l}\text { Incomplete Contracts and } \\
\text { Complexity Costs }\end{array}$ & $\begin{array}{l}\text { Anderlini,-Luca; } \\
\text { Felli,-Leonardo }\end{array}$ & $\begin{array}{l}\text { Theory-and-Decision; 46(1), } \\
\text { February 1999, pp. 23-50. } \\
\end{array}$ & 1999 & Felli & Leonardo & LSE & 0.56 & 1 & 2 & 0.373333333 & \\
\hline Describability and Agency Problems & $\begin{array}{l}\text { Anderlini,-Luca; } \\
\text { Felli,-Leonardo }\end{array}$ & $\begin{array}{l}\text { European-Economic-Review; } \\
\text { 42(1), January 1998, pp. 35- } \\
\text { 59. }\end{array}$ & 1998 & Felli & Leonardo & LSE & 0.84 & 1 & 2 & 0.56 & \\
\hline $\begin{array}{l}\text { Ex-Ante Efficiency of Bankruptcy } \\
\text { Procedures }\end{array}$ & $\begin{array}{l}\text { Cornelli,-Francesca; } \\
\text { Felli,-Leonardo }\end{array}$ & $\begin{array}{l}\text { European-Economic-Review; } \\
\text { 41(3-5), April 1997, pp. 475- } \\
85 .\end{array}$ & 1997 & Felli & Leonardo & LSE & 0.84 & 1 & 2 & 0.56 & \\
\hline $\begin{array}{l}\text { Sistemi di incentivazione della forza } \\
\text { lavoro: Una rassegna dei principali } \\
\text { problemi teorici. }\end{array}$ & \begin{tabular}{|l}
$\begin{array}{l}\text { Felli,-Leonardo; } \\
\text { Ichino,-Andrea }\end{array}$ \\
\end{tabular} & $\begin{array}{l}\text { Politica-Economica; 12(3), } \\
\text { December 1996, pp. 331-61. }\end{array}$ & 1996 & Felli & Leonardo & LSE & 0 & 1 & 2 & 0 & \\
\hline $\begin{array}{l}\text { Learning, Wage Dynamics, and } \\
\text { Firm-Specific Human Capital }\end{array}$ & \begin{tabular}{|l|} 
Felli,-Leonardo; \\
Harris,-Christopher
\end{tabular} & $\begin{array}{l}\text { Journal-of-Political-Economy; } \\
\text { 104(4), August 1996, pp. 838- } \\
68 . \\
\end{array}$ & 1996 & Felli & Leonardo & LSE & 1.95 & 1 & 2 & 1.3 & \\
\hline $\begin{array}{l}\text { Incomplete Written Contracts: } \\
\text { Undescribable States of Nature }\end{array}$ & $\begin{array}{l}\text { Anderlini,-Luca; } \\
\text { Felli,-Leonardo }\end{array}$ & $\begin{array}{l}\text { Quarterly-Journal-of- } \\
\text { Economics; 109(4), Nov. } \\
\text { 1994, pp. 1085-1124. } \\
\end{array}$ & 1994 & Felli & Leonardo & LSE & 3.2 & 1 & 2 & 2.133333333 & \\
\hline $\begin{array}{l}\text { Doveri fiduciari, proprieta e } \\
\text { controllo. }\end{array}$ & \begin{tabular}{|l} 
Barca,-Fabrizio; \\
Felli,-Leonardo
\end{tabular} & $\begin{array}{l}\text { Politica-Economica; 9(3), } \\
\text { December 1993, pp. 397-412. }\end{array}$ & 1993 & Felli & Leonardo & LSE & 0 & 1 & 2 & 0 & \\
\hline $\begin{array}{l}\text { Turnovers and Asymptotic Behavior } \\
\text { of Workers }\end{array}$ & Felli,-Leonardo & $\begin{array}{l}\text { Economics-Letters; 42(1), } \\
\text { 1993, pp. 43-50. }\end{array}$ & 1993 & Felli & Leonardo & LSE & 0.26 & 0.5 & 1 & 0.13 & \\
\hline
\end{tabular}


London School of Economics

\begin{tabular}{|c|c|c|c|c|c|c|c|c|c|c|c|}
\hline & Author(s) & Journal & Year & & & & \begin{tabular}{|r|} 
Impact \\
Factor
\end{tabular} & $\begin{array}{c}\text { Seiten- } \\
\text { korr. }\end{array}$ & Autoren & Impact & personal Impact \\
\hline $\begin{array}{l}\text { Job Matching and the Distribution of } \\
\text { Producer Surplus }\end{array}$ & $\begin{array}{l}\text { Bertola,-Giuseppe; } \\
\text { Felli,-Leonardo }\end{array}$ & $\begin{array}{l}\text { Ricerche-Economiche; 47(1), } \\
\text { March 1993, pp. 65-92. }\end{array}$ & 1993 & Felli & Leonardo & LSE & 0 & 1 & 2 & 0 & L. Felli: 5.056666667 \\
\hline $\begin{array}{l}\text { Some Regulatory Concerns; and an } \\
\text { Incentive Structure for Financial } \\
\text { Regulation }\end{array}$ & $\begin{array}{l}\text { Goodhart,-Charles- } \\
\text { A.-E. }\end{array}$ & $\begin{array}{l}\text { Schweizerische-Zeitschrift-fur- } \\
\text { Volkswirtschaft-undStatistik; } \\
\text { 132(4/2), Dec. 1996, pp. 649- } \\
\text { 50. }\end{array}$ & 1996 & Goodhart & Charles & LSE & 0 & 0.5 & 1 & 0 & \\
\hline $\begin{array}{l}\text { An Incentive Structure for Financial } \\
\text { Regulation }\end{array}$ & $\begin{array}{l}\text { Goodhart,-Charles- } \\
\text { A.-E. }\end{array}$ & $\begin{array}{l}\text { Schweizerische-Zeitschrift-fur- } \\
\text { Volkswirtschaft-undStatistik; } \\
\text { 132(4/2), Dec. 1996, pp. 637- } \\
\text { 47. }\end{array}$ & 1996 & Goodhart & Charles & LSE & 0 & 0.5 & 1 & 0 & \\
\hline Some Regulatory Concerns & $\begin{array}{l}\text { Goodhart,-Charles- } \\
\text { A.-E. }\end{array}$ & $\begin{array}{l}\text { Schweizerische-Zeitschrift-fur- } \\
\text { Volkswirtschaft-undStatistik; } \\
\text { 132(4/2), Dec. 1996, pp. 613- } \\
\text { 35. }\end{array}$ & 1996 & Goodhart & Charles & LSE & 0 & $\overline{1}$ & 1 & 0 & \\
\hline $\begin{array}{l}\text { Microstructural Dynamics in a } \\
\text { Foreign Exchange Electronic } \\
\text { Broking System }\end{array}$ & $\begin{array}{l}\text { Goodhart,-Charles- } \\
\text { A.-E.; Payne,- } \\
\text { Richard-G. }\end{array}$ & $\begin{array}{l}\text { Journal-of-International- } \\
\text { Money-and-Finance; 15(6), } \\
\text { December 1996, pp. 829-52. }\end{array}$ & 1996 & Goodhart & Charles & LSE & 0.49 & 1 & 2 & 0.326666667 & \\
\hline $\begin{array}{l}\text { What Should Central Banks Do? } \\
\text { What Should Be Their } \\
\text { Macroeconomic Objectives and } \\
\text { Operations? }\end{array}$ & $\begin{array}{l}\text { Goodhart,-Charles- } \\
\text { A.-E. }\end{array}$ & $\begin{array}{l}\text { Economic-Journal; 104(427), } \\
\text { November 1994, pp. 1424-36. }\end{array}$ & 1994 & Goodhart & Charles & LSE & 1.21 & 1 & 1 & 1.21 & \\
\hline $\begin{array}{l}\text { Histeresis y fluctuaciones } \\
\text { economicas (Espana, 1970-1994): } \\
\text { Comentario }\end{array}$ & Goodhart,-Charles & $\begin{array}{l}\text { Moneda-y-Credito; 0(202), } \\
\text { 1996, pp. 149-51. }\end{array}$ & 1996 & Goodhart & Charles & LSE & 0 & 0.5 & 1 & 0 & \\
\hline $\begin{array}{l}\text { Central Bank Independence: Book } \\
\text { Reivew }\end{array}$ & Goodhart,-Charles & $\begin{array}{l}\text { Journal-of-International-and- } \\
\text { Comparative-Economics; 4(1), } \\
\text { 1995, pp. 71-82. } \\
\end{array}$ & 1995 & Goodhart & Charles & LSE & 0 & 1 & $\overline{1}$ & 0 & \\
\hline $\begin{array}{l}\text { A Currency Transactions Tax. Why } \\
\text { and How: Discussant to Professor } \\
\text { J. Tobin }\end{array}$ & $\begin{array}{l}\text { Goodhart,-Charles- } \\
\text { A. }\end{array}$ & $\begin{array}{l}\text { Economic-Systems; 20(2-3), } \\
\text { 1996, pp. 91-95. }\end{array}$ & 1996 & Goodhart & Charles & LSE & 0 & 0.5 & 1 & 0 & \\
\hline European Monetary Integration & Goodhart,-Charles & $\begin{array}{l}\text { European-Economic-Review; } \\
40(3-5) \text {, April 1996, pp. 1083- } \\
90 .\end{array}$ & 1996 & Goodhart & Charles & LSE & 0.84 & 0.5 & 1 & 0.42 & \\
\hline $\begin{array}{l}\text { The Rise of China as an Economic } \\
\text { Power }\end{array}$ & $\begin{array}{l}\text { Goodhart,-Charles- } \\
\text { A.-E.; Xu,-C. }\end{array}$ & $\begin{array}{l}\text { National-Institute-Economic- } \\
\text { Review; 0(155), February } \\
\text { 1996, pp. 56-80. }\end{array}$ & 1996 & Goodhart & Charles & LSE & 0 & 1 & 2 & 0 & \\
\hline $\begin{array}{l}\text { The Interaction between the } \\
\text { Frequency of Market Quotations, } \\
\text { Spread and Volatility in the Foreign } \\
\text { Exchange Markets }\end{array}$ & $\begin{array}{l}\text { Demos,-Antonis-A.; } \\
\text { Goodhart,-Charles- } \\
\text { A.-E. }\end{array}$ & $\begin{array}{l}\text { Applied-Economics; 28(3), } \\
\text { March 1996, pp. 377-86. }\end{array}$ & 1996 & Goodhart & Charles & LSE & 0.28 & 0.5 & 2 & 0.093333333 & \\
\hline $\begin{array}{l}\text { Private and Regulatory Soloutions: } \\
\text { Comment: International }\end{array}$ & $\begin{array}{l}\text { Goodhart,-Charles- } \\
\text { A.-E. }\end{array}$ & \begin{tabular}{|l} 
Journal-of-Financial-Services- \\
Research; 9(3-4), December \\
1995, pp. 421-24. \\
\end{tabular} & 1995 & Goodhart & Charles & LSE & 0 & 0.5 & 1 & 0 & \\
\hline $\begin{array}{l}\text { El declive de la banca tradicional: } \\
\text { Implicaciones para la estabilidad } \\
\text { financiera y la politica de regulacion: } \\
\text { Comentario }\end{array}$ & Goodhart,-Charles & $\begin{array}{l}\text { Moneda-y-Credito; 0(200), } \\
\text { 1995, pp. 85-88. }\end{array}$ & 1995 & Goodhart & Charles & LSE & 0 & 0.5 & 1 & 0 & \\
\hline
\end{tabular}


London School of Economics

\begin{tabular}{|c|c|c|c|c|c|c|c|c|c|c|c|}
\hline & Author(s) & Journal & Year & & & & $\begin{array}{l}\text { Impact } \\
\text { Factor }\end{array}$ & \begin{tabular}{|c|} 
Seiten- \\
korr.
\end{tabular} & Autoren & Impact & personal Impact \\
\hline $\begin{array}{l}\text { Should the Functions of Monetary } \\
\text { Policy and Banking Supervision Be } \\
\text { Separated? }\end{array}$ & $\begin{array}{l}\text { Goodhart,-Charles; } \\
\text { Schoenmaker,-Dirk }\end{array}$ & $\begin{array}{l}\text { Oxford-Economic-Papers; } \\
\text { 47(4), October 1995, pp. 539- } \\
60 .\end{array}$ & 1995 & Goodhart & Charles & LSE & 0.74 & 1 & 2 & 0.493333333 & \\
\hline $\begin{array}{l}\text { Real Business Cycle Theory: A } \\
\text { Restatement of Robertsonian } \\
\text { Economics? }\end{array}$ & $\begin{array}{l}\text { Goodhart,-Charles- } \\
\text { A.-E.; Presley,-John }\end{array}$ & $\begin{array}{l}\text { Economic-Notes; 23(2), 1994, } \\
\text { pp. 275-91. }\end{array}$ & 1994 & Goodhart & Charles & LSE & 0 & 1 & 2 & 0 & \\
\hline $\begin{array}{l}\text { The Free Banking Challenge to } \\
\text { Central Banks }\end{array}$ & $\begin{array}{l}\text { Goodhart,-Charles- } \\
\text { A.-E. }\end{array}$ & $\begin{array}{l}\text { Critical-Review; 8(3), Summer } \\
\text { 1994, pp. 411-25. }\end{array}$ & 1994 & Goodhart & Charles & LSE & 0 & 1 & 1 & 0 & \\
\hline $\begin{array}{l}\text { Banks and the Control of } \\
\text { Corporations }\end{array}$ & $\begin{array}{l}\text { Goodhart,-Charles- } \\
\text { A.-E. }\end{array}$ & $\begin{array}{l}\text { Economic-Notes; 23(1), 1994, } \\
\text { pp. 1-18. }\end{array}$ & 1994 & Goodhart & Charles & LSE & 0 & 1 & 1 & 0 & \\
\hline $\begin{array}{l}\text { Banks and the Control of } \\
\text { Corporations }\end{array}$ & Goodhart,-Charles & $\begin{array}{l}\text { Cyprus-Journal-of-Economics; } \\
\text { 6(2), December 1993, pp. 91- } \\
108 .\end{array}$ & 1993 & Goodhart & Charles & LSE & 0 & 0.5 & 1 & 0 & \\
\hline $\begin{array}{l}\text { Game Theory for Central Bankers: } \\
\text { A Report to the Governor of the } \\
\text { Bank of England }\end{array}$ & $\begin{array}{l}\text { Goodhart,-Charles- } \\
\text { A.-E. }\end{array}$ & \begin{tabular}{|l|} 
Journal-of-Economic- \\
Literature; 32(1), March 1994, \\
pp. 101-14.
\end{tabular} & 1994 & Goodhart & Charles & LSE & 3.78 & 1 & 1 & 3.78 & \\
\hline $\begin{array}{l}\text { Institutional Separation between } \\
\text { Supervisory and Monetary Agencies }\end{array}$ & $\begin{array}{l}\text { Goodhart,-Charles- } \\
\text { A.-E.; } \\
\text { Schoenmaker,-Dirk } \\
\end{array}$ & \begin{tabular}{|l|} 
Giornale-degli-Economisti-e- \\
Annali-di-Economia; 51(9-12), \\
Oct.-Dec. 1992, pp. 353-439. \\
\end{tabular} & 1992 & Goodhart & Charles & LSE & 0 & 1 & 2 & 0 & \\
\hline $\begin{array}{l}\text { Central Bank Forex Intervention } \\
\text { Assessed in Continuous Time }\end{array}$ & $\begin{array}{l}\text { Goodhart,-Charles- } \\
\text { A.-E.; Hesse,- } \\
\text { Thomas }\end{array}$ & \begin{tabular}{|l|} 
Journal-of-International- \\
Money-and-Finance; $12(4)$, \\
August 1993, pp. 368-89. \\
\end{tabular} & 1993 & Goodhart & Charles & LSE & 0.49 & 1 & 2 & 0.326666667 & \\
\hline The External Dimension of EMU & $\begin{array}{l}\text { Goodhart,-Charles- } \\
\text { A.-E. }\end{array}$ & $\begin{array}{l}\text { Recherches-Economiques-de } \\
\text { Louvain; 59(1-2), 1993, } \\
\text { pp. 65-80. }\end{array}$ & 1993 & Goodhart & Charles & LSE & 0 & 1 & 1 & 0 & \\
\hline $\begin{array}{l}\text { Testing for Unit Roots with Very } \\
\text { High Frequency Spot Exchange } \\
\text { Rate Data }\end{array}$ & $\begin{array}{l}\text { Goodhart,-C.-A.-E.; } \\
\text { McMahon,-P.-C.; } \\
\text { Ngama,-Y.-L. }\end{array}$ & $\begin{array}{l}\text { Journal-of-Macroeconomics; } \\
\text { 15(3), Summer 1993, pp. 423- } \\
38 .\end{array}$ & 1993 & Goodhart & Charles & LSE & 0.32 & 1 & 3 & 0.16 & \\
\hline $\begin{array}{l}\text { Can We Improve the Structure of } \\
\text { Financial Systems? }\end{array}$ & $\begin{array}{l}\text { Goodhart,-Charles- } \\
\text { A.-E. }\end{array}$ & $\begin{array}{l}\text { European-Economic-Review; } \\
37(2-3) \text {, April 1993, pp. 269- } \\
91 .\end{array}$ & 1993 & Goodhart & Charles & LSE & 0.84 & 1 & 1 & 0.84 & \\
\hline $\begin{array}{l}\text { Does the Forward } \\
\text { Premium/Discount Help to Predict } \\
\text { the Future Change in the Exchange } \\
\text { Rate? }\end{array}$ & $\begin{array}{l}\text { Goodhart,-C.-A.-E.; } \\
\text { McMahon,-P.-C.; } \\
\text { Ngama,-Y.-L. }\end{array}$ & $\begin{array}{l}\text { Scottish-Journal-of-Political- } \\
\text { Economy; 39(2), May 1992, } \\
\text { pp. 129-40. }\end{array}$ & 1992 & Goodhart & Charles & LSE & 0.46 & 1 & 3 & 0.23 & \\
\hline $\begin{array}{l}\text { Why Don't Individuals Speculate in } \\
\text { the Forward Foreign Exchange } \\
\text { Market? }\end{array}$ & $\begin{array}{l}\text { Goodhart,-Charles- } \\
\text { A.-E.; Taylor,-Mark- } \\
\text { P. }\end{array}$ & $\begin{array}{l}\text { Scottish-Journal-of-Political- } \\
\text { Economy; 39(1), February } \\
\text { 1992, pp. 1-13. }\end{array}$ & 1992 & Goodhart & Charles & LSE & 0.46 & 1 & 2 & 0.306666667 & \\
\hline $\begin{array}{l}\text { Economists' Perspectives on the } \\
\text { EMS: A Review Essay }\end{array}$ & $\begin{array}{l}\text { Goodhart,-Charles- } \\
\text { A.-E. }\end{array}$ & $\begin{array}{l}\text { Journal-of-Monetary- } \\
\text { Economics; } 26(3), \text { December } \\
\text { 1990, pp. 471-87. }\end{array}$ & 1990 & Goodhart & Charles & LSE & 1.16 & 1 & 1 & 1.16 & \\
\hline $\begin{array}{l}\text { Every Minute Counts in Financial } \\
\text { Markets }\end{array}$ & $\begin{array}{l}\text { Goodhart,-Charles- } \\
\text { A.-E.; Figliuoli,-L. }\end{array}$ & $\begin{array}{l}\text { Journal-of-International- } \\
\text { Money-and-Finance; 10(1), } \\
\text { March 1991,pp. 23-52. } \\
\end{array}$ & 1991 & Goodhart & Charles & LSE & 0.49 & 1 & 2 & 0.326666667 & $\begin{array}{l}\text { C. Goodhart: } \\
\text { 9.673333333 }\end{array}$ \\
\hline $\begin{array}{l}\text { The Method of Simulated Scores for } \\
\text { the Estimation of LDV Models }\end{array}$ & \begin{tabular}{|l} 
Hajivassiliou,-- \\
Vassilis-A.; \\
McFadden,-Daniel-L.
\end{tabular} & $\begin{array}{l}\text { Econometrica; 66(4), July } \\
\text { 1998, pp. 863-96. }\end{array}$ & 1998 & Hajivassiliou & Vassilis A. & LSE & 2.22 & 1 & 2 & 1.48 & \\
\hline
\end{tabular}


London School of Economics

\begin{tabular}{|c|c|c|c|c|c|c|c|c|c|c|c|}
\hline & Author(s) & Journal & Year & & & & \begin{tabular}{|l} 
Impact \\
Factor \\
\end{tabular} & \begin{tabular}{|c|}
$\begin{array}{c}\text { Seiten- } \\
\text { korr. }\end{array}$ \\
\end{tabular} & Autoren & Impact & personal Impact \\
\hline $\begin{array}{l}\text { Simulation of Multivariate Normal } \\
\text { Rectangle Probabilities and Their } \\
\text { Derivatives: Theoretical and } \\
\text { Computational Results }\end{array}$ & $\begin{array}{l}\text { Hajivassiliou,-V.-A.; } \\
\text { McFadden,-D.-L.; } \\
\text { Ruud,-P. }\end{array}$ & $\begin{array}{l}\text { Journal-of-Econometrics; } \\
72(1-2) \text {, May 1996, pp. 85- } \\
134 .\end{array}$ & 1996 & Hajivassiliou & Vassilis A. & LSE & 1.06 & 1 & 3 & 0.53 & \\
\hline $\begin{array}{l}\text { Duality and Liquidity Constraints } \\
\text { under Uncertainty }\end{array}$ & $\begin{array}{l}\text { Hajivassiliou,- } \\
\text { Vassilis-A.; } \\
\text { loannides,-Yannis- } \\
\text { M. } \\
\end{array}$ & $\begin{array}{l}\text { Journal-of-Economic- } \\
\text { Dynamics-and-Control; 20(6- } \\
\text { 7), June-July 1996, pp. 1177- } \\
\text { 92. } \\
\end{array}$ & 1996 & Hajivassiliou & Vassilis A. & LSE & 0.53 & 1 & 2 & 0.353333333 & \\
\hline $\begin{array}{l}\text { Smooth Unbiased Multivariate } \\
\text { Probability Simulators for Maximum } \\
\text { Likelihood Estimation of Limited } \\
\text { Dependent Variable... }\end{array}$ & $\begin{array}{l}\text { Borsch-Supan,-Axel; } \\
\text { Hajivassiliou,-- } \\
\text { Vassilis-A. }\end{array}$ & $\begin{array}{l}\text { Journal-of-Econometrics; } \\
\text { 58(3), August 1993, pp. } 347- \\
68 .\end{array}$ & 1993 & Hajivassiliou & Vassilis A. & LSE & 1.06 & 1 & 2 & 0.706666667 & $\begin{array}{l}\text { V. A. Hajivassiliou: } \\
3.07\end{array}$ \\
\hline $\begin{array}{l}\text { A Nonparametric Test for Poolability } \\
\text { Using Panel Data }\end{array}$ & $\begin{array}{l}\text { Baltagi,-Badi-H.; } \\
\text { Hidalgo,-Javier; Li,-- } \\
\text { Qi }\end{array}$ & $\begin{array}{l}\text { Journal-of-Econometrics; } \\
\text { 75(2), December 1996, } \\
\text { pp. 345-67. }\end{array}$ & 1996 & Hidalgo & Javier F. & LSE & 1.06 & 1 & 3 & 0.53 & \\
\hline $\begin{array}{l}\text { Testing for Structural Change in a } \\
\text { Long-Memory Environment }\end{array}$ & $\begin{array}{l}\text { Hidalgo,-Javier; } \\
\text { Robinson,-Peter-M. }\end{array}$ & $\begin{array}{l}\text { Journal-of-Econometrics; } \\
70(1) \text {, January 1996, pp. 159- } \\
74 .\end{array}$ & 1996 & Hidalgo & Javier F. & LSE & 1.06 & 1 & 2 & 0.706666667 & \\
\hline $\begin{array}{l}\text { A Nonparametric Conditional } \\
\text { Moment Test for Structural Stability }\end{array}$ & Hidalgo,-Javier & $\begin{array}{l}\text { Econometric-Theory; 11(4), } \\
\text { October 1995, pp. 671-98. }\end{array}$ & 1995 & Hidalgo & Javier F. & LSE & 0.5 & 1 & 1 & 0.5 & \\
\hline $\begin{array}{l}\text { Adaptive Estimation in Time Series } \\
\text { Regression Models with } \\
\text { Heteroskedasticity of Unknown } \\
\text { Form }\end{array}$ & Hidalgo,-Javier & $\begin{array}{l}\text { Econometric-Theory; 8(2), } \\
\text { June 1992, pp. 161-87. }\end{array}$ & 1992 & Hidalgo & Javier F. & LSE & 0.5 & 1 & 1 & 0.5 & $\begin{array}{l}\text { J. F. Hidalgo: } \\
2.236666667\end{array}$ \\
\hline $\begin{array}{l}\text { Regional Policy in an Enlarged } \\
\text { Europe }\end{array}$ & Jackman,-Richard & \begin{tabular}{|l|} 
Oxford-Review -of-Economic- \\
Policy; 11(2), Summer 1995, \\
pp. 113-25.
\end{tabular} & 1995 & Jackman & Richard A. & LSE & 0.29 & 1 & 1 & 0.29 & \\
\hline $\begin{array}{l}\text { Efficiency Considerations in } \\
\text { Enterprise Provision of Social } \\
\text { Benefits }\end{array}$ & Jackman,-Richard & $\begin{array}{l}\text { Economics-of-Transition; 3(2), } \\
\text { June 1995, pp. 251-55. }\end{array}$ & 1995 & Jackman & Richard A. & LSE & 0 & 0.5 & 1 & 0 & \\
\hline $\begin{array}{l}\text { What Can Active Labour Market } \\
\text { Policy Do? }\end{array}$ & Jackman,-Richard & \begin{tabular}{|l|} 
Swedish-Economic-Policy- \\
Review; 1(1-2), Autumn 1994, \\
pp. 221-57.
\end{tabular} & 1994 & Jackman & Richard A. & LSE & 0 & 1 & 1 & 0 & \\
\hline $\begin{array}{l}\text { Economic Policy and Employment } \\
\text { in the Transition Economies of } \\
\text { Central and Eastern Europe: What } \\
\text { Have We Learned? }\end{array}$ & Jackman,-Richard & $\begin{array}{l}\text { International-Labour-Review; } \\
\text { 133(3), 1994, pp. 327-45. }\end{array}$ & 1994 & Jackman & Richard A. & LSE & 0.24 & 1 & 1 & 0.24 & \\
\hline $\begin{array}{l}\text { Mass Unemployment: International } \\
\text { Experience and Lessons for Policy }\end{array}$ & Jackman,-Richard & \begin{tabular}{|l|} 
Finnish-Economic-Papers; \\
6(1), Spring 1993, pp. 5-12. \\
\end{tabular} & 1993 & Jackman & Richard A. & LSE & 0 & 0.5 & 1 & 0 & \\
\hline $\begin{array}{l}\text { Regional Migration versus Regional } \\
\text { Commuting: The Identification of } \\
\text { Housing and Employment Flows }\end{array}$ & $\begin{array}{l}\text { Jackman,-Richard; } \\
\text { Savouri,-Savvas }\end{array}$ & $\begin{array}{l}\text { Scottish-Journal-of-Political- } \\
\text { Economy; 39(3), August 1992, } \\
\text { pp. 272-87. }\end{array}$ & 1992 & Jackman & Richard A. & LSE & 0.46 & 1 & 2 & 0.306666667 & \\
\hline $\begin{array}{l}\text { Regional Migration in Britain: An } \\
\text { Analysis of Gross Flows Using NHS } \\
\text { Central Register Data }\end{array}$ & $\begin{array}{l}\text { Jackman,-Richard; } \\
\text { Savouri,-Savvas }\end{array}$ & $\begin{array}{l}\text { Economic-Journal; 102(415), } \\
\text { November 1992, pp. 1433-50. }\end{array}$ & 1992 & Jackman & Richard A. & LSE & 1.21 & 1 & 2 & 0.806666667 & \\
\hline $\begin{array}{l}\text { Does Long-term Unemployment } \\
\text { Reduce a Person's Chance of a } \\
\text { Job? A Time-Series Test }\end{array}$ & $\begin{array}{l}\text { Jackman,-Richard; } \\
\text { Layard,-Richard }\end{array}$ & $\begin{array}{l}\text { Economica; 58(229), February } \\
\text { 1991, pp. 93-106. }\end{array}$ & 1991 & Jackman & Richard A. & LSE & 0.51 & 1 & 2 & 0.34 & \\
\hline
\end{tabular}


London School of Economics

\begin{tabular}{|c|c|c|c|c|c|c|c|c|c|c|c|}
\hline & Author(s) & Journal & Year & & & & $\begin{array}{c}\text { Impact } \\
\text { Factor }\end{array}$ & $\begin{array}{c}\text { Seiten- } \\
\text { korr. }\end{array}$ & Autoren & Impact & personal Impact \\
\hline $\begin{array}{l}\text { Labour Market Policies and } \\
\text { Unemployment in the OECD }\end{array}$ & $\begin{array}{l}\text { Jackman,-R.; } \\
\text { Pissarides,-C.; } \\
\text { Savouri,-S. }\end{array}$ & $\begin{array}{l}\text { Economic-Policy:-A- } \\
\text { European-Forum; 5(2), } \\
\text { October 1990, pp. 449-90. }\end{array}$ & 1990 & Jackman & Richard A. & LSE & 0 & 1 & 3 & 0 & \\
\hline $\begin{array}{l}\text { The Real Effects of Tax-Based } \\
\text { Incomes Policies }\end{array}$ & $\begin{array}{l}\text { Jackman,-Richard; } \\
\text { Layard,-Richard }\end{array}$ & $\begin{array}{l}\text { Scandinavian-Journal-of- } \\
\text { Economics; 92(2), 1990, } \\
\text { pp. 309-24. }\end{array}$ & 1990 & Jackman & Richard A. & LSE & 0.48 & 1 & 2 & 0.32 & $\begin{array}{l}\text { R. A. Jackman: } \\
2.303333333\end{array}$ \\
\hline $\begin{array}{l}\text { Wealth and Portfolio Composition: } \\
\text { Theory and Evidence }\end{array}$ & $\begin{array}{l}\text { King,-Mervyn-A.; } \\
\text { Leape,-Jonathan-I. }\end{array}$ & $\begin{array}{l}\text { Journal-of-Public-Economics; } \\
\text { 69(2), August 1998, pp. 155- } \\
\text { 93. }\end{array}$ & 1998 & Leape & Jonathan I. & LSE & 0.82 & 1 & 2 & 0.546666667 & J. I. Leape: 0,54666 \\
\hline $\begin{array}{l}\text { The Asymptotic Distribution of } \\
\text { Nonparametric Estimates of the } \\
\text { Lyapunov Exponent for Stochastic } \\
\text { Time Series }\end{array}$ & $\begin{array}{l}\text { Whang,-Yoon-Jae; } \\
\text { Linton,-Oliver }\end{array}$ & $\begin{array}{l}\text { Journal-of-Econometrics; } \\
\text { 91(1), July 1999, pp. 1-42. }\end{array}$ & 1999 & Linton & Oliver & LSE & 1.06 & 1 & 2 & 0.706666667 & \\
\hline $\begin{array}{l}\text { A GARCH Model of the Implied } \\
\text { Volatility of the Swiss Market Index } \\
\text { from Option Prices }\end{array}$ & $\begin{array}{l}\text { Sabbatini,-Michael; } \\
\text { Linton,--Oliver }\end{array}$ & $\begin{array}{l}\text { International-Journal-of- } \\
\text { Forecasting; 14(2), June } \\
\text { 1998, pp. 199-213. }\end{array}$ & 1998 & Linton & Oliver & LSE & 0.42 & 1 & 2 & 0.28 & \\
\hline $\begin{array}{l}\text { An Asymptotic Expansion in the } \\
\text { GARCH }(1,1) \text { Model }\end{array}$ & Linton,-Oliver & $\begin{array}{l}\text { Econometric-Theory; } 13(4), \\
\text { August 1997, pp. 558-81. }\end{array}$ & 1997 & Linton & Oliver & LSE & 0.5 & 1 & 1 & 0.5 & \\
\hline $\begin{array}{l}\text { Second Order Approximation in the } \\
\text { Partially Linear Regression Model }\end{array}$ & Linton,-Oliver & $\begin{array}{l}\text { Econometrica; 63(5), } \\
\text { September 1995, pp. 1079- } \\
1112 .\end{array}$ & 1995 & Linton & Oliver & LSE & 2.22 & 1 & 1 & 2.22 & \\
\hline $\begin{array}{l}\text { Adaptive Estimation in } \mathrm{ARCH} \\
\text { Models }\end{array}$ & Linton,-Oliver & $\begin{array}{l}\text { Econometric-Theory; 9(4), } \\
\text { December 1993, pp. 539-69. }\end{array}$ & 1993 & Linton & Oliver & LSE & 0.5 & 1 & 1 & 0.5 & $\begin{array}{l}\text { O. Linton: } \\
\text { 4.206666667 }\end{array}$ \\
\hline $\begin{array}{l}\text { What Level of Fixed Costs Can } \\
\text { Reconcile Consumption and Stock } \\
\text { Returns? }\end{array}$ & Luttmer,-Erzo-G.-J. & $\begin{array}{l}\text { Journal-of-Political-Economy; } \\
\text { 107(5), October 1999, } \\
\text { pp. 969-97. }\end{array}$ & 1999 & Luttmer & Erzo & LSE & 1.95 & 1 & 1 & 1.95 & \\
\hline $\begin{array}{l}\text { Asset Pricing in Economies with } \\
\text { Frictions }\end{array}$ & Luttmer,-Erzo-G.-J. & $\begin{array}{l}\text { Econometrica; 64(6), } \\
\text { November 1996, pp. 1439-67. }\end{array}$ & 1996 & Luttmer & Erzo & LSE & 2.22 & 1 & 1 & 2.22 & \\
\hline $\begin{array}{l}\text { Econometric Evaluation of Asset } \\
\text { Pricing Models }\end{array}$ & $\begin{array}{l}\text { Hansen,-L.-P.; } \\
\text { Heaton,-J.; Luttmer,-- } \\
\text { E.-G.-J. }\end{array}$ & $\begin{array}{l}\text { Review -of-Financial-Studies; } \\
\text { 8(2), Summer 1995, pp. 237- } \\
\text { 74. }\end{array}$ & 1995 & Luttmer & Erzo & LSE & 1.13 & 1 & 3 & 0.565 & E. Luttmer: 4.735 \\
\hline $\begin{array}{l}\text { The Effects of Minimum Wages on } \\
\text { Employment: Theory and Evidence } \\
\text { from Britain }\end{array}$ & \begin{tabular}{|l|} 
Dickens,-R.; \\
Machin,-S.; \\
Manning,-A. \\
\end{tabular} & $\begin{array}{l}\text { Journal-of-Labor-Economics; } \\
\text { 17(1), January 1999, pp. 1-22. }\end{array}$ & $\mid 1999$ & Manning & Alan & LSE & 1.12 & 1 & 3 & 0.56 & \\
\hline $\begin{array}{l}\text { Comment on B. Holmlund, } \\
\text { "Unemployment Insurance in } \\
\text { Theory and Practice." }\end{array}$ & Manning,-Alan & \begin{tabular}{|l|} 
Scandinavian-Journal-of- \\
Economics; 100(1), March \\
1998, pp. 143-45.
\end{tabular} & 1998 & Manning & Alan & LSE & 0.48 & 0.5 & 1 & 0.24 & \\
\hline $\begin{array}{l}\text { Estimating the Effect of Minimum } \\
\text { Wages on Employment from the } \\
\text { Distribution of Wages: A Critical } \\
\text { View }\end{array}$ & $\begin{array}{l}\text { Dickens,-R.; } \\
\text { Machin,-S.; } \\
\text { Manning,-A. }\end{array}$ & $\begin{array}{l}\text { Labour-Economics; 5(2), June } \\
\text { 1998, pp. 109-34. }\end{array}$ & 1998 & Manning & Alan & LSE & 0 & 1 & 3 & 0 & \\
\hline $\begin{array}{l}\text { Skill-Biassed Change, } \\
\text { Unemployment and Wage Inequality }\end{array}$ & $\begin{array}{l}\text { Gregg,-Paul; } \\
\text { Manning,-Alan }\end{array}$ & $\begin{array}{l}\text { European-Economic-Review; } \\
41(6), \text { June 1997, pp. 1173- } \\
1200 .\end{array}$ & 1997 & Manning & Alan & LSE & 0.84 & 1 & 2 & 0.56 & \\
\hline $\begin{array}{l}\text { Minimum Wages and Economic } \\
\text { Outcomes in Europe }\end{array}$ & $\begin{array}{l}\text { Machin,-Stephen; } \\
\text { Manning,-Alan }\end{array}$ & $\begin{array}{l}\text { European-Economic-Review; } \\
\text { 41(3-5), April 1997, pp. 733- } \\
\text { 42. }\end{array}$ & 1997 & Manning & Alan & LSE & 0.84 & 0.5 & 2 & 0.28 & \\
\hline
\end{tabular}


London School of Economics

\begin{tabular}{|c|c|c|c|c|c|c|c|c|c|c|c|}
\hline & Author(s) & Journal & Year & & & & $\begin{array}{l}\text { Impact } \\
\text { Factor }\end{array}$ & \begin{tabular}{|c|} 
Seiten- \\
korr.
\end{tabular} & Autoren & Impact & personal Impact \\
\hline $\begin{array}{l}\text { Can Supply Create Its Own } \\
\text { Demand? Implications for Rising } \\
\text { Skill Differentials }\end{array}$ & $\begin{array}{l}\text { Machin,-Stephen; } \\
\text { Manning,-Alan }\end{array}$ & $\begin{array}{l}\text { European-Economic-Review; } \\
41(3-5) \text {, April 1997, pp. 507- } \\
16 .\end{array}$ & 1997 & Manning & Alan & LSE & 0.84 & 0.5 & 2 & 0.28 & \\
\hline $\begin{array}{l}\text { Authority in Employment Contracts: } \\
\text { A Bilateral Bargaining Models }\end{array}$ & Manning,-Alan & $\begin{array}{l}\text { Labour-Economics; 3(1), } \\
\text { August 1996, pp. } 1-23 .\end{array}$ & \begin{tabular}{|l|l}
1996 \\
\end{tabular} & Manning & Alan & LSE & 0 & 1 & $\overline{1}$ & 0 & \\
\hline $\begin{array}{l}\text { The Employer Size-Wage Effect: } \\
\text { Can Dynamic Monopsony Provide } \\
\text { an Explanation? }\end{array}$ & $\begin{array}{l}\text { Green,-Francis; } \\
\text { Machin,-Stephen; } \\
\text { Manning,-Alan }\end{array}$ & $\begin{array}{l}\text { Oxford-Economic-Papers; } \\
\text { 48(3), July 1996, pp. 433-55. }\end{array}$ & 1996 & Manning & Alan & LSE & 0.74 & 1 & 3 & 0.37 & \\
\hline $\begin{array}{l}\text { The Economic Impact of Minimum } \\
\text { Wages in Europe }\end{array}$ & Dolado,-Juan et-al. & $\begin{array}{l}\text { Economic-Policy:-A- } \\
\text { European-Forum; 0(23), } \\
\text { October 1996, pp. 317-57. }\end{array}$ & 1996 & Manning & Alan & LSE & 0 & 1 & 3 & 0 & \\
\hline $\begin{array}{l}\text { The Equal Pay Act as an } \\
\text { Experiment to Test Theories of the } \\
\text { Labour Market }\end{array}$ & Manning,-Alan & $\begin{array}{l}\text { Economica; 63(250), May } \\
\text { 1996, pp. 191-212. }\end{array}$ & 1996 & Manning & Alan & LSE & 0.51 & 1 & 1 & \begin{tabular}{|l|l|}
0.51 \\
\end{tabular} & \\
\hline After Wages Councils & $\begin{array}{l}\text { Dickens,-Richard; } \\
\text { Manning,-Alan }\end{array}$ & $\begin{array}{l}\text { New-Economy; 2(4), Winter } \\
\text { 1995, pp. 223-27. }\end{array}$ & 1995 & Manning & Alan & LSE & 0 & 0.5 & 2 & 0 & \\
\hline $\begin{array}{l}\text { Employment and the Introduction of } \\
\text { a Minimum Wage in Britain }\end{array}$ & $\begin{array}{l}\text { Machin,-Stephen; } \\
\text { Manning,-Alan }\end{array}$ & $\begin{array}{l}\text { Economic-Journal; 106(436), } \\
\text { May 1996, pp. 667-76. }\end{array}$ & 1996 & Manning & Alan & LSE & 1.21 & 0.5 & 2 & 0.403333333 & \\
\hline $\begin{array}{l}\text { Developments in Labour Market } \\
\text { Theory and Their Implications for } \\
\text { Macroeconomic Policy }\end{array}$ & Manning,-Alan & $\begin{array}{l}\text { Scottish-Journal-of-Political- } \\
\text { Economy; 42(3), August 1995, } \\
\text { pp. 250-66. }\end{array}$ & 1995 & Manning & Alan & LSE & 0.46 & 1 & 1 & 0.46 & \\
\hline $\begin{array}{l}\text { How Do We Know That Real Wages } \\
\text { Are Too High? }\end{array}$ & Manning,-Alan & $\begin{array}{l}\text { Quarterly-Journal-of- } \\
\text { Economics; } 110(4), \text { November } \\
\text { 1995, pp. 1111-25. }\end{array}$ & 1995 & Manning & Alan & LSE & 3.2 & 1 & 1 & 3.2 & \\
\hline $\begin{array}{l}\text { Minimum Wages and Employment: } \\
\text { A Theoretical Framework with an } \\
\text { Application to the UK Wages } \\
\text { Councils }\end{array}$ & $\begin{array}{l}\text { Dickens,-R.; } \\
\text { Machin,-S.; } \\
\text { Manning,-A. }\end{array}$ & $\begin{array}{l}\text { International-Journal-of- } \\
\text { Manpower; 15(2-3), 1994, } \\
\text { pp. 26-48. }\end{array}$ & 1994 & Manning & Alan & LSE & 0 & 1 & 3 & 0 & \\
\hline $\begin{array}{l}\text { How Robust Is the Microeconomic } \\
\text { Theory of the Trade Union? }\end{array}$ & Manning,-Alan & $\begin{array}{l}\text { Journal-of-Labor-Economics; } \\
\text { 12(3), July 1994, pp. 430-59. }\end{array}$ & 1994 & Manning & Alan & LSE & 1.12 & 1 & 1 & 1.12 & \\
\hline $\begin{array}{l}\text { Pre-strike Ballots and Wage- } \\
\text { Employment Bargaining }\end{array}$ & Manning,-Alan & $\begin{array}{l}\text { Oxford-Economic-Papers; } \\
\text { 45(3), July 1993, pp. 422-39. }\end{array}$ & 1993 & Manning & Alan & LSE & 0.74 & 1 & 1 & 0.74 & \\
\hline $\begin{array}{l}\text { The Effects of Minimum Wages on } \\
\text { Wage Dispersion and Employment: } \\
\text { Evidence from the U.K. Wages } \\
\text { Councils }\end{array}$ & $\begin{array}{l}\text { Machin,-Stephen; } \\
\text { Manning,-Alan }\end{array}$ & $\begin{array}{l}\text { Industrial-and-Labor- } \\
\text { Relations-Review; 47(2), } \\
\text { January 1994, pp. 319-29. }\end{array}$ & 1994 & Manning & Alan & LSE & 0 & 1 & 2 & 0 & \\
\hline $\begin{array}{l}\text { Wage Setting and the Tax System: } \\
\text { Theory and Evidence for the United } \\
\text { Kingdom }\end{array}$ & $\begin{array}{l}\text { Lockwood,-Ben; } \\
\text { Manning,-Alan }\end{array}$ & $\begin{array}{l}\text { Journal-of-Public-Economics; } \\
\text { 52(1), August 1993, pp. 1-29. }\end{array}$ & 1993 & Manning & Alan & LSE & 0.82 & $\overline{1}$ & 2 & 0.546666667 & \\
\hline $\begin{array}{l}\text { A Dynamic Model of Union Power, } \\
\text { Wages and Employment }\end{array}$ & Manning,-Alan & $\begin{array}{l}\text { Scandinavian-Journal-of- } \\
\text { Economics; 95(2), 1993, } \\
\text { pp. 175-93. }\end{array}$ & 1993 & Manning & Alan & LSE & 0.48 & $\overline{1}$ & $\overline{1}$ & 0.48 & \\
\hline $\begin{array}{l}\text { Wage Bargaining and the Phillips } \\
\text { Curve: The Identification and } \\
\text { Specification of Aggregate Wage } \\
\text { Equations }\end{array}$ & Manning,-Alan & $\begin{array}{l}\text { Economic-Journal; 103(416), } \\
\text { January 1993, pp. 98-118. }\end{array}$ & 1993 & Manning & Alan & LSE & 1.21 & 1 & 1 & 1.21 & \\
\hline
\end{tabular}


London School of Economics

\begin{tabular}{|c|c|c|c|c|c|c|c|c|c|c|c|}
\hline & Author(s) & Journal & Year & & & & $\begin{array}{l}\text { Impact } \\
\text { Factor }\end{array}$ & \begin{tabular}{|c|} 
Seiten- \\
korr.
\end{tabular} & Autoren & Impact & personal Impact \\
\hline $\begin{array}{l}\text { Multiple Equilibria in the British } \\
\text { Labour Market: Some Empirical } \\
\text { Evidence }\end{array}$ & Manning,-Alan & $\begin{array}{l}\text { European-Economic-Review; } \\
\text { 36(7), October 1992, } \\
\text { pp. 1333-65. }\end{array}$ & 1992 & Manning & Alan & LSE & 0.84 & 1 & 1 & 0.84 & \\
\hline $\begin{array}{l}\text { Productivity Growth Reconsidered: } \\
\text { Discussion }\end{array}$ & Manning,-Alan & $\begin{array}{l}\text { Economic-Policy:-A- } \\
\text { European-Forum; 0(15), } \\
\text { October 1992, pp. 422-26. }\end{array}$ & 1992 & Manning & Alan & LSE & 0 & 0.5 & $\overline{1}$ & 0 & \\
\hline $\begin{array}{l}\text { Imperfect Labour Markets, the Stock } \\
\text { Market and the Inefficiency of } \\
\text { Capitalism }\end{array}$ & Manning,-Alan & $\begin{array}{l}\text { Oxford-Economic-Papers; } \\
44(2), \text { April 1992, pp. 257-51. }\end{array}$ & 1992 & Manning & Alan & LSE & 0.74 & 0.5 & 1 & 0.37 & \\
\hline $\begin{array}{l}\text { Testing Dynamic Models of Worker } \\
\text { Effort }\end{array}$ & $\begin{array}{l}\text { Machin,-Stephen; } \\
\text { Manning,-Alan }\end{array}$ & $\begin{array}{l}\text { Journal-of-Labor-Economics; } \\
\text { 10(3), July 1992, pp. 288-305. }\end{array}$ & 1992 & Manning & Alan & LSE & 1.12 & 1 & 2 & \begin{tabular}{|l|}
0.746666667 \\
\end{tabular} & \\
\hline $\begin{array}{l}\text { The Determinants of Wage } \\
\text { Pressure: Some Implications of a } \\
\text { Dynamic Model }\end{array}$ & Manning,-Alan & $\begin{array}{l}\text { Economica; 58(231), August } \\
\text { 1991, pp. 325-39. }\end{array}$ & \begin{tabular}{|l|}
1991 \\
\end{tabular} & Manning & Alan & LSE & 0.51 & 1 & 1 & 0.51 & \\
\hline $\begin{array}{l}\text { Tests of Alternative Wage } \\
\text { Employment Bargaining Models } \\
\text { with an Application to the UK } \\
\text { Aggregate Labour Market }\end{array}$ & $\begin{array}{l}\text { Alogoskoufis,-- } \\
\text { George-S.; } \\
\text { Manning,-Alan }\end{array}$ & $\begin{array}{l}\text { European-Economic-Review; } \\
\text { 35(1), January 1991, pp. 23- } \\
\text { 37. }\end{array}$ & 1991 & Manning & Alan & LSE & 0.84 & 1 & 2 & 0.56 & \\
\hline $\begin{array}{l}\text { Imperfect Competition, Multiple } \\
\text { Equilibria and Unemployment Policy }\end{array}$ & Manning,-Alan & $\begin{array}{l}\text { Economic-Journal; 100(400), } \\
\text { Supplement, 1990, pp. 151- } \\
62 .\end{array}$ & 1990 & Manning & Alan & LSE & 1.21 & 1 & $\overline{1}$ & 1.21 & $\begin{array}{l}\text { A. Manning: } \\
15.19666667\end{array}$ \\
\hline $\begin{array}{l}\text { Optimal Debt Contracts and the } \\
\text { Single-Crossing Condition }\end{array}$ & $\begin{array}{l}\text { Faure-Grimaud,-- } \\
\text { Antoine; Mariotti,-- } \\
\text { Thomas } \\
\end{array}$ & $\begin{array}{l}\text { Economics-Letters; 65(1), } \\
\text { October 1999, pp. 85-89. }\end{array}$ & \begin{tabular}{|l|}
1999 \\
\end{tabular} & Mariotti & Thomas & LSE & 0.26 & 0.5 & 2 & 0.086666667 & T. Mariotti: 0,08666 \\
\hline Equilibria in Networks & $\begin{array}{l}\text { Hendricks,-Ken; } \\
\text { Piccione,-Michele; } \\
\text { Tan,-Guofu }\end{array}$ & $\begin{array}{l}\text { Econometrica; 67(6), } \\
\text { November 1999, pp. 1407-34. }\end{array}$ & 1999 & Piccione & Michele & LSE & 2.22 & 1 & 3 & 1.11 & \\
\hline $\begin{array}{l}\text { Evolutionary Stability in Alternating- } \\
\text { Offers Bargaining Games }\end{array}$ & $\begin{array}{l}\text { Binmore,-K.; } \\
\text { Piccione,-M.; } \\
\text { Samuelson,-L. }\end{array}$ & $\begin{array}{l}\text { Journal-of-Economic-Theory; } \\
\text { 80(2), June 1998, pp. 257-91. }\end{array}$ & 1998 & Piccione & Michele & LSE & 0.93 & 1 & 3 & 0.465 & \\
\hline $\begin{array}{l}\text { The Absent-Minded Driver's } \\
\text { Paradox: Synthesis and Responses }\end{array}$ & $\begin{array}{l}\text { Piccione,-Michele; } \\
\text { Rubinstein,-Ariel }\end{array}$ & $\begin{array}{l}\text { Games-and-Economic- } \\
\text { Behavior; 20(1), July 1997, } \\
\text { pp. 121-30. }\end{array}$ & 1997 & Piccione & Michele & LSE & 0.6 & 0.5 & 2 & 0.2 & \\
\hline $\begin{array}{l}\text { On the Interpretation of Decision } \\
\text { Problems with Imperfect Recall }\end{array}$ & $\begin{array}{l}\text { Piccione,-Michele; } \\
\text { Rubinstein,-Ariel }\end{array}$ & $\begin{array}{l}\text { Games-and-Economic- } \\
\text { Behavior; 20(1), July 1997, } \\
\text { pp. 3-24. }\end{array}$ & 1997 & Piccione & \begin{tabular}{|l|} 
Michele \\
\end{tabular} & LSE & 0.6 & 1 & 2 & 0.4 & \\
\hline $\begin{array}{l}\text { Entry and Exit in Hub-Spoke } \\
\text { Networks }\end{array}$ & $\begin{array}{l}\text { Hendricks,-Ken; } \\
\text { Piccione,-Michele; } \\
\text { Tan,-Guofu }\end{array}$ & $\begin{array}{l}\text { RAND-Journal-of-Economics; } \\
\text { 28(2), Summer 1997, pp. 291- } \\
303 \text {. }\end{array}$ & \begin{tabular}{|l|}
1997 \\
\end{tabular} & Piccione & Michele & LSE & 1.13 & 1 & 3 & 0.565 & \\
\hline $\begin{array}{l}\text { A Simple Model of Expert and Non- } \\
\text { expert Bidding in First-Price } \\
\text { Auctions }\end{array}$ & $\begin{array}{l}\text { Piccione,-Michele; } \\
\text { Tan,-Guofu }\end{array}$ & $\begin{array}{l}\text { Journal-of-Economic-Theory; } \\
70(2) \text {, August 1996, pp. 501- } \\
15 .\end{array}$ & 1996 & Piccione & Michele & LSE & 0.93 & 1 & 2 & 0.62 & \\
\hline $\begin{array}{l}\text { Cost-Reducing Investment, Optimal } \\
\text { Procurement and Implementation by } \\
\text { Auctions }\end{array}$ & $\begin{array}{l}\text { Piccione,-Michele; } \\
\text { Tan,-Guofu }\end{array}$ & $\begin{array}{l}\text { International-Economic- } \\
\text { Review; 37(3), August 1996, } \\
\text { pp. 663-85. }\end{array}$ & 1996 & Piccione & Michele & LSE & 0.69 & 1 & 2 & 0.46 & \\
\hline $\begin{array}{l}\text { The Economics of Hubs: The Case } \\
\text { of Monopoly }\end{array}$ & $\begin{array}{l}\text { Hendricks,-Ken; } \\
\text { Piccione,-Michele; } \\
\text { Tan,-Guofu }\end{array}$ & $\begin{array}{l}\text { Review-of-Economic-Studies; } \\
62(1) \text {, January 1995, pp. 83- } \\
\text { 99. }\end{array}$ & 1995 & Piccione & Michele & LSE & 1.52 & 1 & 3 & 0.76 & \\
\hline
\end{tabular}


London School of Economics

\begin{tabular}{|c|c|c|c|c|c|c|c|c|c|c|c|}
\hline & Author(s) & Journal & Year & & & & \begin{tabular}{|c|} 
Impact \\
Factor
\end{tabular} & $\begin{array}{c}\text { Seiten- } \\
\text { korr. }\end{array}$ & Autoren & Impact & personal Impact \\
\hline $\begin{array}{l}\text { Finite Automata Play a Repeated } \\
\text { Extensive Game }\end{array}$ & \begin{tabular}{|l} 
Piccione,-Michele; \\
Rubinstein,-Ariel
\end{tabular} & $\begin{array}{l}\text { Journal-of-Economic-Theory; } \\
61(1) \text {, October 1993, pp. 160- } \\
68 .\end{array}$ & 1993 & Piccione & Michele & LSE & 0.93 & 0.5 & 2 & 0.31 & \\
\hline $\begin{array}{l}\text { Finite Automata Equilibria with } \\
\text { Discounting }\end{array}$ & Piccione,-Michele & $\begin{array}{l}\text { Journal-of-Economic-Theory; } \\
\text { 56(1), February 1992, } \\
\text { pp. 180-93. }\end{array}$ & 1992 & Piccione & Michele & LSE & 0.93 & 1 & 1 & 0.93 & M. Piccione: 5.82 \\
\hline $\begin{array}{l}\text { Policy Influences on } \\
\text { Unemployment: The European } \\
\text { Experience }\end{array}$ & $\begin{array}{l}\text { Pissarides,-- } \\
\text { Christopher-A. }\end{array}$ & $\begin{array}{l}\text { Scottish-Journal-of-Political- } \\
\text { Economy; 46(4), September } \\
\text { 1999, pp. 389-418. }\end{array}$ & 1999 & Pissarides & Christopher A. & LSE & 0.46 & 1 & 1 & 0.46 & \\
\hline $\begin{array}{l}\text { Unemployment Responses to 'Skill- } \\
\text { Biased' Technology Shocks: The } \\
\text { Role of Labour Market Policy }\end{array}$ & $\begin{array}{l}\text { Mortensen,-Dale-T.; } \\
\text { Pissarides,- } \\
\text { Christopher-A. } \\
\end{array}$ & $\begin{array}{l}\text { Economic-Journal; 109(455), } \\
\text { April 1999, pp. 242-65. }\end{array}$ & 1999 & Pissarides & Christopher A. & LSE & 1.21 & 1 & 2 & 0.8066666667 & \\
\hline $\begin{array}{l}\text { The Need for Labor-Market } \\
\text { Flexibility in a European Economic } \\
\text { and Monetary Union }\end{array}$ & \begin{tabular}{|l|} 
Pissarides,- \\
Christopher-A.
\end{tabular} & $\begin{array}{l}\text { Swedish-Economic-Policy- } \\
\text { Review; 4(2), Autumn 1997, } \\
\text { pp. 513-45. }\end{array}$ & 1997 & Pissarides & Christopher A. & LSE & 0 & 1 & 1 & 0 & \\
\hline $\begin{array}{l}\text { The Impact of Employment Tax } \\
\text { Cuts on Unemployment and Wages; } \\
\text { the Role of Unemployment Benefits } \\
\text { and ... }\end{array}$ & $\begin{array}{l}\text { Pissarides,-- } \\
\text { Christopher-A. }\end{array}$ & $\begin{array}{l}\text { European-Economic-Review; } \\
\text { 42(1), January 1998, pp. 155- } \\
\text { 83. }\end{array}$ & 1998 & Pissarides & Christopher A. & LSE & 0.84 & 1 & 1 & 0.84 & \\
\hline $\begin{array}{l}\text { Learning by Trading and the } \\
\text { Returns to Human Capital in } \\
\text { Developing Countries }\end{array}$ & $\begin{array}{l}\text { Pissarides,-- } \\
\text { Christopher-A. }\end{array}$ & \begin{tabular}{|l|} 
World-Bank-Economic- \\
Review; 11(1), January 1997, \\
pp. 17-32.
\end{tabular} & 1997 & Pissarides & Christopher A. & LSE & 1.32 & 1 & 1 & 1.32 & \\
\hline $\begin{array}{l}\text { Search Unemployment with On-the- } \\
\text { Job Search }\end{array}$ & $\begin{array}{l}\text { Pissarides,-- } \\
\text { Christopher-A. }\end{array}$ & $\begin{array}{l}\text { Review-of-Economic-Studies; } \\
\text { 61(3), July 1994, pp. 457-75. }\end{array}$ & 1994 & Pissarides & Christopher A. & LSE & 1.52 & 1 & 1 & 1.52 & \\
\hline $\begin{array}{l}\text { Job Creation and Job Destruction in } \\
\text { the Theory of Unemployment }\end{array}$ & $\begin{array}{l}\text { Mortensen,-Dale-T.; } \\
\text { Pissarides,-- } \\
\text { Christopher-A. }\end{array}$ & $\begin{array}{l}\text { Review-of-Economic-Studies; } \\
\text { 61(3), July 1994, pp. 397-415. }\end{array}$ & 1994 & Pissarides & Christopher A. & LSE & 1.52 & 1 & 2 & 1.013333333 & \\
\hline $\begin{array}{l}\text { On-the-Job Search: Some } \\
\text { Empirical Evidence from Britain }\end{array}$ & $\begin{array}{l}\text { Pissarides,- } \\
\text { Christopher-A.; } \\
\text { Wadsworth,-- } \\
\text { Jonathan } \\
\end{array}$ & $\begin{array}{l}\text { European-Economic-Review; } \\
\text { 38(2), February 1994, } \\
\text { pp. 385-401. }\end{array}$ & 1994 & Pissarides & Christopher A. & LSE & 0.84 & 1 & 2 & 0.56 & \\
\hline $\begin{array}{l}\text { Loss of Skill during Unemployment } \\
\text { and the Persistence of Employment } \\
\text { Shocks }\end{array}$ & $\begin{array}{l}\text { Pissarides,- } \\
\text { Christopher-A. }\end{array}$ & $\begin{array}{l}\text { Quarterly-Journal-of- } \\
\text { Economics; } 107(4) \text {, November } \\
\text { 1992, pp. 1371-91. }\end{array}$ & 1992 & Pissarides & Christopher A. & LSE & 3.2 & 1 & 1 & 3.2 & \\
\hline $\begin{array}{l}\text { Macroeconomic Adjustment and } \\
\text { Poverty in Selected Industrial } \\
\text { Countries }\end{array}$ & $\begin{array}{l}\text { Pissarides,- } \\
\text { Christopher-A. }\end{array}$ & $\begin{array}{l}\text { World-Bank-Economic- } \\
\text { Review; 5(2), May 1991, } \\
\text { pp. 207-29. }\end{array}$ & 1991 & Pissarides & Christopher A. & LSE & 1.32 & 1 & 1 & 1.32 & \\
\hline $\begin{array}{l}\text { Regional Migration, Wages and } \\
\text { Unemployment: Empirical Evidence } \\
\text { and Implications for Policy }\end{array}$ & $\begin{array}{l}\text { Pissarides,- } \\
\text { Christopher-A.; } \\
\text { McMaster,-lan }\end{array}$ & $\begin{array}{l}\text { Oxford-Economic-Papers,-N.- } \\
\text { S.; 42(4), October 1990, } \\
\text { pp. 812-31. }\end{array}$ & 1990 & Pissarides & Christopher A. & LSE & 0.74 & 1 & 2 & 0.493333333 & \\
\hline $\begin{array}{l}\text { Real Wages and Unemployment in } \\
\text { Australia }\end{array}$ & \begin{tabular}{|l|} 
Pissarides,- \\
Christopher-A.
\end{tabular} & $\begin{array}{l}\text { Economica; 58(229), February } \\
\text { 1991, pp. 35-55. }\end{array}$ & 1991 & Pissarides & Christopher A. & LSE & 0.51 & 1 & 1 & 0.51 & $\begin{array}{l}\text { C. A. Pissarides: } \\
12.04333333\end{array}$ \\
\hline $\begin{array}{l}\text { Hierarchies of Processors with } \\
\text { Endogenous Capacity }\end{array}$ & Prat,-Andrea & $\begin{array}{l}\text { Journal-of-Economic-Theory; } \\
77(1) \text {, November 1997, } \\
\text { pp. 214-22. }\end{array}$ & 1997 & Prat & Andrea & LSE & 0.93 & 1 & 1 & 0.93 & \\
\hline $\begin{array}{l}\text { Shared Knowledge vs Diversified } \\
\text { Knowledge in Teams }\end{array}$ & Prat,-Andrea & $\begin{array}{l}\text { Journal-of-the-Japanese-and- } \\
\text { International-Economies; } \\
\text { 10(2), June 1996, pp. 181-95. }\end{array}$ & 1996 & Prat & Andrea & LSE & 0.36 & 1 & 1 & 0.36 & A. Prat: 1.29 \\
\hline
\end{tabular}


London School of Economics

\begin{tabular}{|c|c|c|c|c|c|c|c|c|c|c|c|}
\hline & Author(s) & Journal & Year & & & & $\begin{array}{l}\text { Impact } \\
\text { Factor }\end{array}$ & \begin{tabular}{|c|}
$\begin{array}{c}\text { Seiten- } \\
\text { korr. }\end{array}$ \\
\end{tabular} & Autoren & Impact & personal Impact \\
\hline $\begin{array}{l}\text { The Federal Reserve's Response to } \\
\text { Aggregate Demand and Aggregate } \\
\text { Supply Shocks: Evidence of a } \\
\text { Partisan ... }\end{array}$ & $\begin{array}{l}\text { Gamber,-Edward-N.; } \\
\text { Hakes,-David-R. }\end{array}$ & $\begin{array}{l}\text { Southern-Economic-Journal; } \\
63(3) \text {, January 1997, pp. } 680 \text { - } \\
91 .\end{array}$ & \begin{tabular}{|l|}
1997 \\
\end{tabular} & Quah & Danny & LSE & 0.39 & 1 & 3 & 0.195 & \\
\hline $\begin{array}{l}\text { Convergence Empirics across } \\
\text { Economies with (Some) Capital } \\
\text { Mobility }\end{array}$ & Quah,-Danny-T. & $\begin{array}{l}\text { Journal-of-Economic-Grow th; } \\
\text { 1(1), March 1996, pp. 95-124. }\end{array}$ & 1996 & Quah & Danny & LSE & 0 & 1 & 1 & 0 & \\
\hline $\begin{array}{l}\text { Empirics for Economic Growth and } \\
\text { Convergence }\end{array}$ & Quah,-Danny-T. & $\begin{array}{l}\text { European-Economic-Review; } \\
\text { 40(6), June 1996, pp. 1353- } \\
75 .\end{array}$ & 1996 & Quah & Danny & LSE & 0.84 & 1 & 1 & 0.84 & \\
\hline $\begin{array}{l}\text { Twin Peaks: Growth and } \\
\text { Convergence in Models of } \\
\text { Distribution Dynamics }\end{array}$ & Quah,-Danny-T. & $\begin{array}{l}\text { Economic-Journal; 106(437), } \\
\text { July 1996, pp. 1045-55. }\end{array}$ & 1996 & Quah & Danny & LSE & 1.21 & 1 & 1 & 1.21 & \\
\hline $\begin{array}{l}\text { Regional Convergence Clusters } \\
\text { across Europe }\end{array}$ & Quah,-Danny-T. & $\begin{array}{l}\text { European-Economic-Review; } \\
40(3-5) \text {, April 1996, pp. 951- } \\
58 .\end{array}$ & 1996 & Quah & Danny & LSE & 0.84 & 0.5 & 1 & 0.42 & \\
\hline $\begin{array}{l}\text { Aggregate and Regional } \\
\text { Disaggregate Fluctuations }\end{array}$ & Quah,-Danny-T. & $\begin{array}{l}\text { Empirical-Economics; 21(1), } \\
\text { 1996, pp. 137-59. }\end{array}$ & 1996 & Quah & Danny & LSE & 0 & 1 & 1 & 0 & \\
\hline $\begin{array}{l}\text { Empirical Estimates of the Short- } \\
\text { Run Aggregate Supply and Demand } \\
\text { Curves for the Post-War U.S. } \\
\text { Economy }\end{array}$ & Gamber,-Edward-N. & $\begin{array}{l}\text { Southern-Economic-Journal; } \\
\text { 62(4), April 1996, pp. 856-72. }\end{array}$ & 1996 & Quah & Danny & LSE & 0.39 & 1 & 2 & 0.26 & \\
\hline $\begin{array}{l}\text { Business Cycle Empirics: } \\
\text { Calibration and Estimation: An } \\
\text { Introduction }\end{array}$ & Quah,-Danny-T. & \begin{tabular}{|l|} 
Economic-Journal; 105(433), \\
November 1995, pp. 1594-96.
\end{tabular} & 1995 & Quah & Danny & LSE & 1.21 & 0.5 & 1 & 0.605 & \\
\hline $\begin{array}{l}\text { Misinterpreting the Dynamic Effects } \\
\text { of Aggregate Demand and Supply } \\
\text { Disturbances }\end{array}$ & Quah,-Danny & $\begin{array}{l}\text { Economics-Letters; 49(3), } \\
\text { September 1995, pp. 247-50. }\end{array}$ & 1995 & Quah & Danny & LSE & 0.26 & 0.5 & 1 & 0.13 & \\
\hline Measuring Core Inflation? & \begin{tabular}{|l} 
Quah,-Danny; \\
Vahey,-Shaun-P.
\end{tabular} & \begin{tabular}{|l|} 
Economic-Journal; 105(432), \\
September 1995, pp. 1130-44.
\end{tabular} & 1995 & Quah & Danny & LSE & 1.21 & 1 & 2 & 0.806666667 & \\
\hline $\begin{array}{l}\text { La riqueza de las regiones. } \\
\text { Evidencia y teorias sobre } \\
\text { crecimiento regional y } \\
\text { convergencia: Comentario }\end{array}$ & Quah,-Danny & $\begin{array}{l}\text { Moneda-y-Credito; 0(198), } \\
\text { 1994, pp. 55-74. }\end{array}$ & 1994 & Quah & Danny & LSE & 0 & 1 & 1 & 0 & \\
\hline $\begin{array}{l}\text { One Business Cycle and One Trend } \\
\text { from (Many,) Many Disaggregates }\end{array}$ & Quah,-Danny & $\begin{array}{l}\text { European-Economic-Review; } \\
\text { 38(3-4), April 1994, pp. 605- } \\
\text { 13. }\end{array}$ & 1994 & Quah & Danny & LSE & 0.84 & 0.5 & 1 & 0.42 & \\
\hline $\begin{array}{l}\text { Exploiting Cross-Section Variation } \\
\text { for Unit Root Inference in Dynamic } \\
\text { Data }\end{array}$ & Quah,-Danny & $\begin{array}{l}\text { Economics-Letters; 44(1-2), } \\
\text { 1994, pp. 9-19. }\end{array}$ & 1994 & Quah & Danny & LSE & 0.26 & 1 & 1 & 0.26 & \\
\hline $\begin{array}{l}\text { Galton's Fallacy and Tests of the } \\
\text { Convergence Hypothesis }\end{array}$ & Quah,-Danny & $\begin{array}{l}\text { Scandinavian-Journal-of- } \\
\text { Economics; 95(4), December } \\
\text { 1993, pp. 427-43. } \\
\end{array}$ & 1993 & Quah & Danny & LSE & 0.48 & 1 & 1 & 0.48 & \\
\hline $\begin{array}{l}\text { Testing for Common Features: } \\
\text { Comment }\end{array}$ & Quah,-Danny & $\begin{array}{l}\text { Journal-of-Business-and- } \\
\text { Economic-Statistics; 11(4), } \\
\text { October 1993, pp. 386-90. }\end{array}$ & 1993 & Quah & Danny & LSE & 1.24 & 0.5 & 1 & 0.62 & \\
\hline
\end{tabular}


London School of Economics

\begin{tabular}{|c|c|c|c|c|c|c|c|c|c|c|c|}
\hline & Author(s) & Journal & Year & & & & \begin{tabular}{r|} 
Impact \\
Factor
\end{tabular} & $\begin{array}{c}\text { Seiten- } \\
\text { korr. }\end{array}$ & Autoren & Impact & personal Impact \\
\hline $\begin{array}{l}\text { The Sources of Business Cycles: A } \\
\text { Monetarist Interpretation }\end{array}$ & Roberts,-John-M. & $\begin{array}{l}\text { International-Economic- } \\
\text { Review; 34(4), November } \\
\text { 1993, pp. 923-34. }\end{array}$ & 1993 & Quah & Danny & LSE & 0.69 & 1 & 2 & 0.46 & \\
\hline $\begin{array}{l}\text { Empirical Cross-Section Dynamics } \\
\text { in Economic Growth }\end{array}$ & Quah,-Danny & $\begin{array}{l}\text { European-Economic-Review; } \\
\text { 37(2-3), April 1993, pp. 426- } \\
\text { 34. }\end{array}$ & 1993 & Quah & Danny & LSE & 0.84 & 0.5 & 1 & 0.42 & \\
\hline $\begin{array}{l}\text { The Dynamic Effects of Aggregate } \\
\text { Demand and Supply Disturbances: } \\
\text { Reply }\end{array}$ & $\begin{array}{l}\text { Blanchard,-Olivier- } \\
\text { Jean; Quah,-Danny }\end{array}$ & $\begin{array}{l}\text { American-Economic-Review; } \\
\text { 83(3), June 1993, pp. 653-58. }\end{array}$ & 1993 & Quah & Danny & LSE & 1.74 & 0.5 & 2 & 0.58 & \\
\hline $\begin{array}{l}\text { The Relative Importance of } \\
\text { Permanent and Transitory } \\
\text { Components: Identification and } \\
\text { Some Theoretical Bounds }\end{array}$ & Quah,-Danny & $\begin{array}{l}\text { Econometrica; 60(1), January } \\
\text { 1992, pp. 107-18. }\end{array}$ & 1992 & Quah & Danny & LSE & 2.22 & 1 & 1 & 2.22 & \\
\hline $\begin{array}{l}\text { Permanent and Transitory } \\
\text { Movements in Labor Income: An } \\
\text { Explanation for "Excess } \\
\text { Smoothness" in Consumption }\end{array}$ & Quah,-Danny & \begin{tabular}{|l|} 
Journal-of-Political-Economy; \\
98(3), June 1990, pp. 449-75.
\end{tabular} & 1990 & Quah & Danny & LSE & 1.95 & 1 & 1 & 1.95 & \\
\hline $\begin{array}{l}\text { An Improved Rate for Non-negative } \\
\text { Definite Consistent Covariance } \\
\text { Matrix Estimation with } \\
\text { Heterogeneous Dependent Data } \\
\end{array}$ & Quah,-Danny & $\begin{array}{l}\text { Economics-Letters; 33(2), } \\
\text { June 1990, pp. 133-40. }\end{array}$ & 1990 & Quah & Danny & LSE & 0.26 & 0.5 & 1 & 0.13 & $\begin{array}{l}\text { D. Quah: } \\
\text { 12.00666667 }\end{array}$ \\
\hline Speculative Securities & $\begin{array}{l}\text { Marin,-Jose-M.; } \\
\text { Rahi,-Rohit }\end{array}$ & \begin{tabular}{|l|} 
Economic-Theory; 14(3), \\
November 1999, pp. 653-668.
\end{tabular} & 1999 & Rahi & Rohit & LSE & 0.5 & 1 & 2 & 0.333333333 & \\
\hline $\begin{array}{l}\text { Adverse Selection and Security } \\
\text { Design }\end{array}$ & Rahi,-Rohit & $\begin{array}{l}\text { Review-of-Economic-Studies; } \\
\text { 63(2), April 1996, pp. 287-300. }\end{array}$ & 1996 & Rahi & Rohit & LSE & 1.52 & 1 & 1 & 1.52 & \\
\hline $\begin{array}{l}\text { Optimal Incomplete Markets with } \\
\text { Asymmetric Information }\end{array}$ & Rahi,-Rohit & $\begin{array}{l}\text { Journal-of-Economic-Theory; } \\
65(1), \text { February } 1995, \\
\text { pp. } 171-97 .\end{array}$ & 1995 & Rahi & Rohit & LSE & 0.93 & 1 & 1 & 0.93 & \\
\hline $\begin{array}{l}\text { Financial Market Innovation and } \\
\text { Security Design: An Introduction }\end{array}$ & $\begin{array}{l}\text { Duffie,-Darrell; Rahi,- } \\
\text { Rohit }\end{array}$ & $\begin{array}{l}\text { Journal-of-Economic-Theory; } \\
65(1), \text { February 1995, pp. 1- } \\
42 .\end{array}$ & 1995 & Rahi & Rohit & LSE & 0.93 & 1 & 2 & 0.62 & \\
\hline $\begin{array}{l}\text { Partially Revealing Rational } \\
\text { Expectations Equilibria with Nominal } \\
\text { Assets }\end{array}$ & Rahi,-Rohit & $\begin{array}{l}\text { Journal-of-Mathematical- } \\
\text { Economics; 24(2), 1995, } \\
\text { pp. 137-46. }\end{array}$ & 1995 & Rahi & Rohit & LSE & 0.37 & 0.5 & 1 & 0.185 & R. Rahi: 3.255 \\
\hline $\begin{array}{l}\text { Dynamic Comparative Advantage } \\
\text { and the Welfare Effects of Trade }\end{array}$ & Redding,-Stephen & $\begin{array}{l}\text { Oxford-Economic-Papers; } \\
51(1) \text {, January 1999, pp. } 15- \\
39 .\end{array}$ & 1999 & Redding & Stephen & LSE & 0.74 & 1 & 1 & 0.74 & \\
\hline $\begin{array}{l}\text { The Low -Skill, Low -Quality Trap: } \\
\text { Strategic Complementarities } \\
\text { between Human Capital and R\&D }\end{array}$ & Redding,-Stephen & $\begin{array}{l}\text { Economic-Journal; 106(435), } \\
\text { March 1996, pp. 458-70. }\end{array}$ & 1996 & Redding & Stephen & LSE & 1.21 & 1 & 1 & 1.21 & S. Redding: 1.95 \\
\hline A Nonparametric Test for I(0). & $\begin{array}{l}\text { Lobato,-Ignacio-N.; } \\
\text { Robinson,-Peter-M. }\end{array}$ & \begin{tabular}{|l|} 
Review-of-Economic-Studies; \\
65(3), July 1998, pp. 475-95.
\end{tabular} & 1998 & Robinson & Peter M. & LSE & 1.52 & 1 & 2 & 1.013333333 & \\
\hline $\begin{array}{l}\text { Testing for Structural Change in a } \\
\text { Long-Memory Environment }\end{array}$ & $\begin{array}{l}\text { Hidalgo,-Javier; } \\
\text { Robinson,-Peter-M. }\end{array}$ & $\begin{array}{l}\text { Journal-of-Econometrics; } \\
\text { 70(1), January 1996, pp. 159- } \\
74 .\end{array}$ & 1996 & Robinson & Peter M. & LSE & 1.06 & 1 & 2 & 0.706666667 & \\
\hline $\begin{array}{l}\text { Nonparametric and Semiparametric } \\
\text { Methods for Economic Research }\end{array}$ & $\begin{array}{l}\text { Delgado,-Miguel-A.; } \\
\text { Robinson,-Peter-M. }\end{array}$ & $\begin{array}{l}\text { Journal-of-Economic-Surveys; } \\
\text { 6(3), 1992, pp. 201-49. }\end{array}$ & 1992 & Robinson & Peter M. & LSE & 0 & 1 & 2 & 0 & P. M. Robinson: 1.72 \\
\hline
\end{tabular}


London School of Economics

\begin{tabular}{|c|c|c|c|c|c|c|c|c|c|c|c|}
\hline & Author(s) & Journal & Year & & & & \begin{tabular}{|c|} 
Impact \\
Factor \\
\end{tabular} & $\begin{array}{c}\text { Seiten- } \\
\text { korr. }\end{array}$ & Autoren & Impact & personal Impact \\
\hline $\begin{array}{l}\text { Ethnic Wage Differences in } \\
\text { Malaysia: Parametric and } \\
\text { Semiparametric Estimation of the } \\
\text { Chinese-Malay Wage Gap } \\
\end{array}$ & $\begin{array}{l}\text { Schafgans,-Marcia- } \\
\text { M.-A. }\end{array}$ & $\begin{array}{l}\text { Journal-of-Applied- } \\
\text { Econometrics; 13(5), Sept.- } \\
\text { Oct. 1998, pp. 481-504. }\end{array}$ & 1998 & Schafgans & Marcia & LSE & 0.72 & 1 & 1 & 0.72 & \\
\hline $\begin{array}{l}\text { Semiparametric Estimation of the } \\
\text { Intercept of a Sample Selection } \\
\text { Model }\end{array}$ & $\begin{array}{l}\text { Andrews,-Donald- } \\
\text { W.-K.; Schafgans,-- } \\
\text { Marcia-M.-A. }\end{array}$ & $\begin{array}{l}\text { Review-of-Economic-Studies; } \\
65(3) \text {, July 1998, pp. 497-517. }\end{array}$ & 1998 & Schafgans & Marcia & LSE & 1.52 & 1 & 2 & 1.013333333 & \\
\hline $\begin{array}{l}\text { The Tail Index of Exchange Rate } \\
\text { Returns }\end{array}$ & $\begin{array}{l}\text { Koedijk,-K.-G.; } \\
\text { Schafgans,-M.-M.- } \\
\text { A.; de-Vries,-C.-G. } \\
\end{array}$ & \begin{tabular}{|l|} 
Journal-of-International- \\
Economics; $29(1-2)$, August \\
1990, pp. 93-108. \\
\end{tabular} & 1990 & Schafgans & Marcia & LSE & 0.8 & 1 & 3 & 0.4 & $\begin{array}{l}\text { M. Schafgans: } \\
2.133333333\end{array}$ \\
\hline $\begin{array}{l}\text { Competition, Entry and the Social } \\
\text { Returns to Infrastructure in } \\
\text { Transition Economies }\end{array}$ & $\begin{array}{l}\text { Aghion,-Philippe; } \\
\text { Schankerman,-Mark }\end{array}$ & $\begin{array}{l}\text { Economics-of-Transition; 7(1), } \\
\text { 1999, pp. 79-101. }\end{array}$ & 1999 & Schankerman & Mark & LSE & 0 & 1 & 2 & 0 & \\
\hline $\begin{array}{l}\text { Patent Renewals and R\&D } \\
\text { Incentives }\end{array}$ & $\begin{array}{l}\text { Cornelli,-Francesca; } \\
\text { Schankerman,-Mark }\end{array}$ & $\begin{array}{l}\text { RAND-Journal-of-Economics; } \\
30(2) \text {, Summer 1999, pp. 197- } \\
213 .\end{array}$ & 1999 & Schankerman & Mark & LSE & 1.13 & 1 & 2 & 0.753333333 & \\
\hline $\begin{array}{l}\text { How Valuable Is Patent Protection? } \\
\text { Estimates by Technology Field }\end{array}$ & Schankerman,-Mark & $\begin{array}{l}\text { RAND-Journal-of-Economics; } \\
\text { 29(1), Spring 1998, pp. 77- } \\
107 .\end{array}$ & 1998 & Schankerman & Mark & LSE & 1.13 & 1 & 1 & 1.13 & \\
\hline $\begin{array}{l}\text { Enterprise Restructuring and Social } \\
\text { Benefits }\end{array}$ & $\begin{array}{l}\text { Commander,-Simon; } \\
\text { Schankerman,-Mark }\end{array}$ & $\begin{array}{l}\text { Economics-of-Transition; 5(1), } \\
\text { May 1997, pp. 1-24. }\end{array}$ & 1997 & Schankerman & Mark & LSE & 0 & 1 & 2 & 0 & \\
\hline $\begin{array}{l}\text { Symmetric Regulation for } \\
\text { Competitive Telecommunications }\end{array}$ & Schankerman,-Mark & $\begin{array}{l}\text { Information-Economics-and- } \\
\text { Policy; 8(1), March 1996, } \\
\text { pp. 3-23. }\end{array}$ & 1996 & Schankerman & Mark & LSE & 0 & 1 & 1 & 0 & $\begin{array}{l}\text { M. Schankerman: } \\
1.883333333\end{array}$ \\
\hline $\begin{array}{l}\text { Financial Fragility, Heterogeneous } \\
\text { Agents, and Aggregate } \\
\text { Fluctuations: Evidence form a Panel } \\
\text { of US Firms }\end{array}$ & $\begin{array}{l}\text { Stanca,-L.; Delli- } \\
\text { Gatti,-D.; Gallegati,-- } \\
\text { M. }\end{array}$ & $\begin{array}{l}\text { Applied-Financial-Economics; } \\
\text { 9(1), February 1999, pp. 87- } \\
\text { 99. }\end{array}$ & 1999 & Stanca & Luca & LSE & 0 & 1 & 3 & 0 & \\
\hline $\begin{array}{l}\text { The Dynamic Relation between } \\
\text { Financial Positions and Investment: } \\
\text { Evidence from Company Account } \\
\text { Data }\end{array}$ & $\begin{array}{l}\text { Stanca,-Luca; } \\
\text { Gallegati,-Mauro }\end{array}$ & $\begin{array}{l}\text { Industrial-and-Corporate- } \\
\text { Change; 8(3), September } \\
\text { 1999, pp. 551-72. }\end{array}$ & 1999 & Stanca & Luca & LSE & 0 & 1 & 2 & 0 & \\
\hline $\begin{array}{l}\text { Le fluttuazioni economiche in Italia } \\
\text { (1861-1992): Un riesame } \\
\text { dell'ipotesi del ciclo rappresentativo. }\end{array}$ & Stanca,-Luca-Matteo & $\begin{array}{l}\text { Rivista-di-Politica-Economica; } \\
\text { 89(3), March 1999, pp. 3-44. }\end{array}$ & 1999 & Stanca & Luca & LSE & 0 & 1 & 1 & 0 & \\
\hline $\begin{array}{l}\text { The Historical Properties of Italian } \\
\text { Macroeconomic Fluctuations: Are } \\
\text { Business Cycles All Alike? }\end{array}$ & $\begin{array}{l}\text { Stanca,-Luca; } \\
\text { Gallegati,-Mauro }\end{array}$ & $\begin{array}{l}\text { Rivista-Internazionale-di- } \\
\text { Scienze-Sociali; 106(2), April- } \\
\text { June 1998, pp. 119-37. } \\
\end{array}$ & 1998 & Stanca & Luca & LSE & 0 & 1 & 2 & 0 & \\
\hline $\begin{array}{l}\text { The Postwar Stabilization of } \\
\text { Economic Fluctuations: Evidence } \\
\text { from a Chain Index of Italian GDP }\end{array}$ & Stanca,-Luca & $\begin{array}{l}\text { Rivista-Internazionale-di- } \\
\text { Scienze-Sociali; 106(1), Jan.- } \\
\text { March 1998, pp. 89-114. }\end{array}$ & 1998 & Stanca & Luca & LSE & 0 & 1 & 1 & 0 & \\
\hline $\begin{array}{l}\text { Regolarita empiriche del ciclo } \\
\text { economico per l'economia italiana } \\
(1960-1993) \text {. }\end{array}$ & Stanca,-Luca-Matteo & \begin{tabular}{|l|} 
Giornale-degli-Economisti-e- \\
Annali-di-Economia; 55(2), \\
April-June 1996, pp. 211-40. \\
\end{tabular} & 1996 & Stanca & Luca & LSE & 0 & 1 & 1 & 0 & L. Stanca: 0 \\
\hline One Smart Agent & Sutton,-John & $\begin{array}{l}\text { RAND-Journal-of-Economics; } \\
28(4) \text {, Winter 1997, pp. 605- } \\
28 \text {. }\end{array}$ & 1997 & Sutton & John & LSE & 1.13 & 1 & 1 & 1.13 & \\
\hline
\end{tabular}


London School of Economics

\begin{tabular}{|c|c|c|c|c|c|c|c|c|c|c|c|}
\hline & Author(s) & Journal & Year & & & & $\begin{array}{l}\text { Impact } \\
\text { Factor }\end{array}$ & $\begin{array}{c}\text { Seiten- } \\
\text { korr. }\end{array}$ & Autoren & Impact & personal Impact \\
\hline History Matters. So What? & Sutton,-John & $\begin{array}{l}\text { Journal-of-the-Economics-of- } \\
\text { Business; } 1(1), 1994, \text { pp. } 41- \\
44 .\end{array}$ & 1994 & Sutton & John & LSE & 0 & 0.5 & 1 & 0 & \\
\hline Gibrat's Legacy & Sutton,-John & $\begin{array}{l}\text { Journal-of-Economic- } \\
\text { Literature; 35(1), March 1997, } \\
\text { pp. 40-59. }\end{array}$ & 1997 & Sutton & John & LSE & 3.78 & 1 & 1 & 3.78 & \\
\hline Technology and Market Structure & Sutton,-John & $\begin{array}{l}\text { European-Economic-Review; } \\
40(3-5) \text {, April 1996, pp. 511- } \\
30 .\end{array}$ & 1996 & Sutton & John & LSE & 0.84 & 1 & 1 & 0.84 & \\
\hline $\begin{array}{l}\text { Echoes of Edgeworth: The Problem } \\
\text { of Indeterminacy }\end{array}$ & Sutton,-John & $\begin{array}{l}\text { European-Economic-Review; } \\
37(2-3) \text {, April 1993, pp. 491- } \\
99 .\end{array}$ & 1993 & Sutton & John & LSE & 0.84 & 0.5 & 1 & 0.42 & \\
\hline $\begin{array}{l}\text { Much Ado about Auctions: Some } \\
\text { Introductory Remarks }\end{array}$ & Sutton,-John & $\begin{array}{l}\text { European-Economic-Review; } \\
37(2-3) \text {, April 1993, pp. 317- } \\
19 .\end{array}$ & 1993 & Sutton & John & LSE & 0.84 & 0.5 & 1 & 0.42 & \\
\hline $\begin{array}{l}\text { Multiproduct Firms and Market } \\
\text { Structure }\end{array}$ & $\begin{array}{l}\text { Shaked,-Avner; } \\
\text { Sutton,-John }\end{array}$ & $\begin{array}{l}\text { Rand-Journal-of-Economics; } \\
\text { 21(1), Spring 1990, pp. 45-62. }\end{array}$ & 1990 & Sutton & John & LSE & 1.13 & 1 & 2 & 0.753333333 & \\
\hline $\begin{array}{l}\text { Explaining Everything, Explaining } \\
\text { Nothing? Game Theoretic Models } \\
\text { in Industrial Economics }\end{array}$ & Sutton,-John & $\begin{array}{l}\text { European-Economic-Review; } \\
34(2-3) \text {, May 1990, pp. 505- } \\
12 .\end{array}$ & 1990 & Sutton & John & LSE & 0.84 & 0.5 & 1 & 0.42 & $\begin{array}{l}\text { J. Sutton: } \\
\text { 7.763333333 }\end{array}$ \\
\hline $\begin{array}{l}\text { The International Division of } \\
\text { Industries: Clustering and } \\
\text { Comparative Advantage in a Multi- } \\
\text { industry Model }\end{array}$ & $\begin{array}{l}\text { Venables,-Anthony- } \\
\text { J. }\end{array}$ & $\begin{array}{l}\text { Scandinavian-Journal-of- } \\
\text { Economics; 101(4), December } \\
\text { 1999, pp. 495-513. }\end{array}$ & 1999 & Venables & Anthony J. & LSE & 0.48 & 1 & 1 & 0.48 & \\
\hline $\begin{array}{l}\text { But Why Does Geography Matter, } \\
\text { and Which Geography Matters? }\end{array}$ & $\begin{array}{l}\text { Venables,-Anthony- } \\
\text { J. }\end{array}$ & \begin{tabular}{|l} 
International-Regional- \\
Science-Review; 22(2), \\
August 1999, pp. 238-41.
\end{tabular} & 1999 & Venables & Anthony J. & LSE & 0 & 0.5 & 1 & 0 & \\
\hline $\begin{array}{l}\text { Fragmentation and Multinational } \\
\text { Production }\end{array}$ & $\begin{array}{l}\text { Venables,-Anthony- } \\
\text { J. }\end{array}$ & $\begin{array}{l}\text { European-Economic-Review; } \\
43(4-6) \text {, April 1999, pp. 935- } \\
45 .\end{array}$ & 1999 & Venables & Anthony J. & LSE & 0.84 & 1 & 1 & 0.84 & \\
\hline $\begin{array}{l}\text { Agglomeration and Economic } \\
\text { Development: Import Substitution } \\
\text { vs. Trade Liberalisation }\end{array}$ & \begin{tabular}{|l} 
Puga,-Diego; \\
Venables,-Anthony- \\
J. \\
\end{tabular} & $\begin{array}{l}\text { Economic-Journal; 109(455), } \\
\text { April 1999, pp. 292-311. }\end{array}$ & 1999 & Venables & Anthony J. & LSE & 1.21 & 1 & 2 & 0.806666667 & \\
\hline $\begin{array}{l}\text { The Assessment: Trade and } \\
\text { Location }\end{array}$ & $\begin{array}{l}\text { Venables,-Anthony- } \\
\text { J. }\end{array}$ & $\begin{array}{l}\text { Oxford-Review -of-Economic- } \\
\text { Policy; 14(2), Summer 1998, } \\
\text { pp. 1-6. }\end{array}$ & 1998 & Venables & Anthony J. & LSE & 0.29 & 0.5 & $\overline{1}$ & 0.145 & \\
\hline $\begin{array}{l}\text { Multinational Firms and the New } \\
\text { Trade Theory }\end{array}$ & \begin{tabular}{|l|} 
Markusen,-James- \\
R.; Venables,- \\
Anthony-J. \\
\end{tabular} & $\begin{array}{l}\text { Journal-of-International- } \\
\text { Economics; 46(2), December } \\
\text { 1998, pp. 183-203. }\end{array}$ & 1998 & Venables & Anthony J. & LSE & 0.8 & 1 & 2 & 0.533333333 & \\
\hline $\begin{array}{l}\text { Foreign Direct Investment as a } \\
\text { Catalyst for Industrial Development }\end{array}$ & $\begin{array}{l}\text { Markusen,-James- } \\
\text { R.; Venables,- } \\
\text { Anthony-J. } \\
\end{array}$ & $\begin{array}{l}\text { European-Economic-Review; } \\
\text { 43(2), February 1999, } \\
\text { pp. 335-56. }\end{array}$ & 1999 & Venables & Anthony J. & LSE & 0.84 & 1 & 2 & 0.56 & \\
\hline $\begin{array}{l}\text { Trading Arrangements and } \\
\text { Industrial Development }\end{array}$ & $\begin{array}{l}\text { Puga,-Diego; } \\
\text { Venables,-Anthony- } \\
\text { J. }\end{array}$ & $\begin{array}{l}\text { World-Bank-Economic- } \\
\text { Review; 12(2), May 1998, } \\
\text { pp. 221-49. }\end{array}$ & 1998 & Venables & Anthony J. & LSE & 1.32 & 1 & 2 & 0.88 & \\
\hline $\begin{array}{l}\text { The Role of Multinational Firms in } \\
\text { the Wage-Gap Debate }\end{array}$ & \begin{tabular}{|l|} 
Markusen,-James- \\
R.; Venables,- \\
Anthony-J.
\end{tabular} & $\begin{array}{l}\text { Review-of-International- } \\
\text { Economics; 5(4), November } \\
\text { 1997, pp. 435-51. }\end{array}$ & 1997 & Venables & Anthony J. & LSE & 0 & 1 & 2 & 0 & \\
\hline
\end{tabular}


London School of Economics

\begin{tabular}{|c|c|c|c|c|c|c|c|c|c|c|c|}
\hline & Author(s) & Journal & Year & & & & $\begin{array}{l}\text { Impact } \\
\text { Factor }\end{array}$ & $\begin{array}{c}\text { Seiten- } \\
\text { korr. }\end{array}$ & Autoren & Impact & personal Impact \\
\hline $\begin{array}{l}\text { Preferential Trading Arrangements } \\
\text { and Industrial Location }\end{array}$ & $\begin{array}{l}\text { Puga,-Diego; } \\
\text { Venables,-Anthony- } \\
\text { J. }\end{array}$ & $\begin{array}{l}\text { Journal-of-International- } \\
\text { Economics; 43(3-4), } \\
\text { November 1997, pp. 347-68. }\end{array}$ & 1997 & Venables & Anthony J. & LSE & 0.8 & 1 & 2 & 0.533333333 & \\
\hline $\begin{array}{l}\text { Localization of Industry and Trade } \\
\text { Performance }\end{array}$ & $\begin{array}{l}\text { Venables,-Anthony- } \\
\text { J. }\end{array}$ & $\begin{array}{l}\text { Oxford-Review -of-Economic- } \\
\text { Policy; 12(3), Autumn 1996, } \\
\text { pp. 52-60. }\end{array}$ & 1996 & Venables & Anthony J. & LSE & 0.29 & 0.5 & 1 & 0.145 & \\
\hline $\begin{array}{l}\text { The Spread of Industry: Spatial } \\
\text { Agglomeration in Economic } \\
\text { Development }\end{array}$ & $\begin{array}{l}\text { Puga,-Diego; } \\
\text { Venables,-Anthony- } \\
\text { J. }\end{array}$ & $\begin{array}{l}\text { Journal-of-the-Japanese-and- } \\
\text { International-Economies; } \\
\text { 10(4), December 1996, } \\
\text { pp. 440-64. }\end{array}$ & 1996 & Venables & Anthony J. & LSE & 0.36 & 1 & 2 & 0.24 & \\
\hline $\begin{array}{l}\text { Integration, Specialization, and } \\
\text { Adjustment }\end{array}$ & $\begin{array}{l}\text { Krugman,-Paul; } \\
\text { Venables,-Anthony- } \\
\text { J. }\end{array}$ & $\begin{array}{l}\text { European-Economic-Review; } \\
40(3-5) \text {, April 1996, pp. 959- } \\
67 .\end{array}$ & 1996 & Venables & Anthony J. & LSE & 0.84 & 0.5 & 2 & 0.28 & \\
\hline $\begin{array}{l}\text { Trade Policy, Cumulative } \\
\text { Causation, and Industrial } \\
\text { Development }\end{array}$ & $\begin{array}{l}\text { Venables,-Anthony- } \\
\text { J. }\end{array}$ & $\begin{array}{l}\text { Journal-of-Development- } \\
\text { Economics; 49(1), April 1996, } \\
\text { pp. 179-97. }\end{array}$ & 1996 & Venables & Anthony J. & LSE & 0.66 & 1 & 1 & 0.66 & \\
\hline $\begin{array}{l}\text { Equilibrium Locations of Vertically } \\
\text { Linked Industries }\end{array}$ & $\begin{array}{l}\text { Venables,-Anthony- } \\
\text { J. }\end{array}$ & $\begin{array}{l}\text { International-Economic- } \\
\text { Review; 37(2), May 1996, } \\
\text { pp. 341-59. }\end{array}$ & 1996 & Venables & Anthony J. & LSE & 0.69 & 1 & 1 & 0.69 & \\
\hline $\begin{array}{l}\text { Globalization and the Inequality of } \\
\text { Nations }\end{array}$ & $\begin{array}{l}\text { Krugman,-Paul-R.; } \\
\text { Venables,-Anthony- } \\
\text { J. }\end{array}$ & $\begin{array}{l}\text { Quarterly-Journal-of- } \\
\text { Economics; } 110(4), \text { November } \\
\text { 1995, pp. 857-80. }\end{array}$ & 1995 & Venables & Anthony J. & LSE & 3.2 & 1 & 2 & 2.133333333 & \\
\hline $\begin{array}{l}\text { International Trade, Factor Mobility } \\
\text { and Trade Costs }\end{array}$ & $\begin{array}{l}\text { Norman,-Victor-D.; } \\
\text { Venables,-Anthony- } \\
\text { J. } \\
\end{array}$ & $\begin{array}{l}\text { Economic-Journal; 105(433), } \\
\text { November 1995, pp. 1488- } \\
1504 .\end{array}$ & 1995 & Venables & Anthony J. & LSE & 1.21 & 1 & 2 & 0.806666667 & \\
\hline $\begin{array}{l}\text { Economic Integration and the } \\
\text { Location of Firms }\end{array}$ & $\begin{array}{l}\text { Venables,-Anthony- } \\
\text { J. }\end{array}$ & \begin{tabular}{|l} 
American-Economic-Review; \\
85(2), May 1995, pp. 296-300.
\end{tabular} & 1995 & Venables & Anthony J. & LSE & 1.74 & 0.5 & 1 & 0.87 & \\
\hline $\begin{array}{l}\text { Economic Integration and Industrial } \\
\text { Agglomeration }\end{array}$ & $\begin{array}{l}\text { Venables,-Anthony- } \\
\text { J. }\end{array}$ & $\begin{array}{l}\text { Economic-and-Social-Review; } \\
\text { 26(1), October 1994, pp. 1-17. }\end{array}$ & 1994 & Venables & Anthony J. & LSE & 0.16 & 1 & 1 & 0.16 & \\
\hline $\begin{array}{l}\text { International Migration, Capital } \\
\text { Mobility and Transitional Dynamics }\end{array}$ & $\begin{array}{l}\text { Baldwin,-Richard; } \\
\text { Venables,-Anthony- } \\
\text { J. }\end{array}$ & $\begin{array}{l}\text { Economica; 61(243), August } \\
\text { 1994, pp. 285-300. }\end{array}$ & 1994 & Venables & Anthony J. & LSE & 0.51 & 1 & 2 & 0.34 & \\
\hline $\begin{array}{l}\text { Integration and the Export } \\
\text { Behaviour of Firms: Trade Costs, } \\
\text { Trade Volumes and Welfare }\end{array}$ & $\begin{array}{l}\text { Venables,-Anthony- } \\
\text { J. }\end{array}$ & $\begin{array}{l}\text { Weltwirtschaftliches-Archiv; } \\
\text { 130(1), 1994, pp. 118-32. }\end{array}$ & 1994 & Venables & Anthony J. & LSE & 0.45 & 1 & 1 & 0.45 & \\
\hline $\begin{array}{l}\text { Tariffs and Subsidies with Price } \\
\text { Competition and Integrated } \\
\text { Markets: The Mixed Strategy } \\
\text { Equilibria }\end{array}$ & $\begin{array}{l}\text { Venables,-Anthony- } \\
\text { J. }\end{array}$ & $\begin{array}{l}\text { Oxford-Economic-Papers; } \\
\text { 46(1), January 1994, pp. 30- } \\
44 .\end{array}$ & 1994 & Venables & Anthony J. & LSE & 0.74 & 1 & 1 & 0.74 & \\
\hline $\begin{array}{l}\text { Integracion y competitividad de la } \\
\text { industria periferica }\end{array}$ & $\begin{array}{l}\text { Krugman,-Paul-R.; } \\
\text { Venables,-Anthony- } \\
\text { J. }\end{array}$ & $\begin{array}{l}\text { Estudios-Economicos; 5(2), } \\
\text { July-Dec. 1990, pp. 263-86. }\end{array}$ & 1990 & Venables & Anthony J. & LSE & 0 & $\overline{1}$ & 2 & 0 & \\
\hline $\begin{array}{l}\text { Economic Integration and Market } \\
\text { Access }\end{array}$ & $\begin{array}{l}\text { Smith,-Alasdair; } \\
\text { Venables,-Anthony- } \\
\text { J. }\end{array}$ & $\begin{array}{l}\text { European-Economic-Review; } \\
\text { 35(2-3), April 1991, pp. 388- } \\
\text { 95. }\end{array}$ & 1991 & Venables & Anthony J. & LSE & 0.84 & 0.5 & 2 & 0.28 & \\
\hline $\begin{array}{l}\text { Microeconomic Implications of } \\
\text { Exchange Rate Variations }\end{array}$ & $\begin{array}{l}\text { Venables,-Anthony- } \\
\text { J. }\end{array}$ & $\begin{array}{l}\text { Oxford-Review -of-Economic- } \\
\text { Policy; 6(3), Autumn 1990, } \\
\text { pp. 18-27. }\end{array}$ & 1990 & Venables & Anthony J. & LSE & 0.29 & 0.5 & $\overline{1}$ & 0.145 & \\
\hline
\end{tabular}


London School of Economics

\begin{tabular}{|c|c|c|c|c|c|c|c|c|c|c|c|}
\hline & Author(s) & Journal & Year & & & & \begin{tabular}{|l} 
Impact \\
Factor
\end{tabular} & $\begin{array}{l}\text { Seiten- } \\
\text { korr. }\end{array}$ & Autoren & Impact & personal Impact \\
\hline $\begin{array}{l}\text { International Capacity Choice and } \\
\text { National Market Games }\end{array}$ & $\begin{array}{l}\text { Venables,-Anthony- } \\
\text { J. }\end{array}$ & $\begin{array}{l}\text { Journal-of-International- } \\
\text { Economics; 29(1-2), August } \\
\text { 1990, pp. 23-42. }\end{array}$ & 1990 & Venables & Anthony J. & LSE & 0.8 & 1 & 1 & 0.8 & \\
\hline $\begin{array}{l}\text { The Economic Integration of } \\
\text { Oligopolistic Markets }\end{array}$ & $\begin{array}{l}\text { Venables,-Anthony- } \\
\text { J. }\end{array}$ & $\begin{array}{l}\text { European-Economic-Review; } \\
\text { 34(4), June 1990, pp. 753-69. }\end{array}$ & 1990 & Venables & Anthony J. & LSE & 0.84 & 1 & 1 & 0.84 & $\begin{array}{l}\text { A. J. Venables: } \\
14.35833333 \\
\end{array}$ \\
\hline $\begin{array}{l}\text { Evaluating the Economic Impact of } \\
\text { Planning Controls in the United } \\
\text { Kingdom: Some Implications for } \\
\text { Housing }\end{array}$ & $\begin{array}{l}\text { Monk,-Sarah; } \\
\text { Whitehead,-- } \\
\text { Christine-M.-E. }\end{array}$ & $\begin{array}{l}\text { Land-Economics; 75(1), } \\
\text { February 1999, pp. 74-93. }\end{array}$ & 1999 & Whitehead & Christine M. E. & LSE & 0.69 & 1 & 2 & 0.46 & \\
\hline $\begin{array}{l}\text { Land Supply and Housing: A Case- } \\
\text { Study }\end{array}$ & $\begin{array}{l}\text { Monk,-Sarah; } \\
\text { Whitehead,-- } \\
\text { Christine-M.-E. }\end{array}$ & $\begin{array}{l}\text { Housing-Studies; 11(3), July } \\
\text { 1996, pp. 407-23. }\end{array}$ & 1996 & Whitehead & Christine M. E. & LSE & 0 & 1 & 2 & 0 & \\
\hline $\begin{array}{l}\text { Editorial: Housing Economics--An } \\
\text { Evolving Agenda }\end{array}$ & $\begin{array}{l}\text { Maclennan,-Duncan; } \\
\text { Whitehead,-- } \\
\text { Christine-M.-E. }\end{array}$ & $\begin{array}{l}\text { Housing-Studies; 11(3), July } \\
\text { 1996, pp. 341-44. }\end{array}$ & 1996 & Whitehead & Christine M. E. & LSE & 0 & 0.5 & 2 & 0 & \\
\hline Private Renting in the 1990s & $\begin{array}{l}\text { Whitehead,- } \\
\text { Christine-M.-E. }\end{array}$ & $\begin{array}{l}\text { Housing-Studies; } 11(1) \\
\text { January 1996, pp. 7-12. }\end{array}$ & 1996 & Whitehead & Christine M. E. & LSE & 0 & 0.5 & 1 & 0 & \\
\hline $\begin{array}{l}\text { Liberalisation and Deregulation in } \\
\text { Urban Housing Markets }\end{array}$ & $\begin{array}{l}\text { Whitehead,-- } \\
\text { Christine-M.-E. }\end{array}$ & $\begin{array}{l}\text { Estudios-de-Economia; 23(0), } \\
\text { Special Issue 1996, pp. 25-55. }\end{array}$ & 1996 & Whitehead & Christine M. E. & LSE & 0 & 1 & 1 & 0 & \\
\hline $\begin{array}{l}\text { Understanding Structural } \\
\text { Adjustment of the Mortgage Market } \\
\text { in the United States: Lessons from } \\
\text { the Protracted ... }\end{array}$ & $\begin{array}{l}\text { Whitehead,-- } \\
\text { Christine-M.-E. }\end{array}$ & $\begin{array}{l}\text { Housing-Policy-Debate; 5(3), } \\
\text { 1994, pp. 231-40. }\end{array}$ & 1994 & Whitehead & Christine M. E. & LSE & 0 & 0.5 & $\overline{1}$ & 0 & \\
\hline $\begin{array}{l}\text { Understanding Structural } \\
\text { Adjustment of the Mortgage Market } \\
\text { in the United States: Lessons from } \\
\text { the Protracted ... }\end{array}$ & $\begin{array}{l}\text { Megbolugbe,-Isaac- } \\
\text { F.; Whitehead,-- } \\
\text { Christine-M.-E. }\end{array}$ & $\begin{array}{l}\text { Housing-Policy-Debate; 5(3), } \\
\text { 1994, pp. 219-30. }\end{array}$ & 1994 & Whitehead & Christine M. E. & LSE & 0 & 1 & 2 & 0 & \\
\hline $\begin{array}{l}\text { Privatizing Housing: An } \\
\text { Assessment of U.K. Experience }\end{array}$ & \begin{tabular}{|l} 
Whitehead,-- \\
Christine-M.-E.
\end{tabular} & $\begin{array}{l}\text { Housing-Policy-Debate; 4(1), } \\
\text { 1993, pp. 101-39. }\end{array}$ & 1993 & Whitehead & Christine M. E. & LSE & 0 & 1 & 1 & 0 & \\
\hline $\begin{array}{l}\text { From Need to Affordability: An } \\
\text { Analysis of UK Housing Objectives }\end{array}$ & $\begin{array}{l}\text { Whitehead,-- } \\
\text { Christine-M.-E. }\end{array}$ & $\begin{array}{l}\text { Urban-Studies; 28(6), } \\
\text { December 1991, pp. 871-87. }\end{array}$ & 1991 & Whitehead & Christine M. E. & LSE & 1.07 & 1 & $\overline{1}$ & 1.07 & $\begin{array}{l}\text { C. M. E. Whitehead } \\
1.53\end{array}$ \\
\hline $\begin{array}{l}\text { Why Is China Different from Eastern } \\
\text { Europe? Perspectives from } \\
\text { Organization Theory }\end{array}$ & \begin{tabular}{|l|} 
Qian,-Yingyi; \\
Roland,-Gerard; Xu,- \\
Chenggang \\
\end{tabular} & $\begin{array}{l}\text { European-Economic-Review; } \\
\text { 43(4-6), April 1999, pp. 1085- } \\
94 .\end{array}$ & 1999 & $\mathrm{Xu}$ & Chenggang & LSE & 0.84 & 0.5 & 3 & 0.21 & \\
\hline $\begin{array}{l}\text { Financial Institutions and the } \\
\text { Financial Crisis in East Asia }\end{array}$ & $\begin{array}{l}\text { Huang,-Haizhou; } \\
\text { Xu,-Chenggang }\end{array}$ & $\begin{array}{l}\text { European-Economic-Review; } \\
\text { 43(4-6), April 1999, pp. 903- } \\
14 .\end{array}$ & 1999 & $\mathrm{Xu}$ & Chenggang & LSE & 0.84 & $\overline{1}$ & 2 & 0.56 & \\
\hline $\begin{array}{l}\text { Institutions, Innovations, and } \\
\text { Growth }\end{array}$ & $\begin{array}{l}\text { Huang,-Haizhou; } \\
\text { Xu,-Chenggang }\end{array}$ & $\begin{array}{l}\text { American-Economic-Review; } \\
\text { 89(2), May 1999, pp. 438-43. }\end{array}$ & 1999 & $\mathrm{Xu}$ & Chenggang & LSE & 1.74 & 0.5 & 2 & 0.58 & \\
\hline $\begin{array}{l}\text { Soft Budget Constraint and the } \\
\text { Optimal Choices of Research and } \\
\text { Development Projects Financing }\end{array}$ & $\begin{array}{l}\text { Huang,-Haizhou; } \\
\text { Xu,-Chenggang }\end{array}$ & $\begin{array}{l}\text { Journal-of-Comparative- } \\
\text { Economics; } 26(1), \text { March } \\
\text { 1998, pp. 62-79. }\end{array}$ & 1998 & $\mathrm{Xu}$ & Chenggang & LSE & 1.27 & 1 & 2 & 0.846666667 & \\
\hline $\begin{array}{l}\text { Innovation and Bureaucracy under } \\
\text { Soft and Hard Budget Constraints }\end{array}$ & $\begin{array}{l}\text { Qian,-Yingyi; Xu,-- } \\
\text { Chenggang }\end{array}$ & $\begin{array}{l}\text { Review -of-Economic-Studies; } \\
65(1) \text {, January 1998, pp. 151- } \\
64 .\end{array}$ & 1998 & $\mathrm{Xu}$ & Chenggang & LSE & 1.52 & 1 & 2 & 1.013333333 & \\
\hline
\end{tabular}


London School of Economics

\begin{tabular}{|c|c|c|c|c|c|c|c|c|c|c|c|}
\hline & Author(s) & Journal & Year & & & & $\begin{array}{l}\text { Impact } \\
\text { Factor }\end{array}$ & $\begin{array}{c}\text { Seiten- } \\
\text { korr. }\end{array}$ & Autoren & Impact & personal Impact \\
\hline $\begin{array}{l}\text { Comments on: "The Soft Budget } \\
\text { Constraint in China." }\end{array}$ & Xu,-Chenggang & $\begin{array}{l}\text { Japan-and-the-World- } \\
\text { Economy; 9(1), March 1997, } \\
\text { pp. 13-15. }\end{array}$ & 1997 & $\mathrm{Xu}$ & Chenggang & LSE & 0.1 & 0.5 & 1 & 0.05 & \\
\hline $\begin{array}{l}\text { Profit-Sharing and Financial } \\
\text { Performance in the Chinese State } \\
\text { Enterprises: Evidence from Panel } \\
\text { Data }\end{array}$ & $\begin{array}{l}\text { Zhuang,-Juzhong; } \\
\text { Xu,-Chenggang }\end{array}$ & $\begin{array}{l}\text { Economics-of-Planning; 29(3), } \\
\text { 1996, pp. 205-22. }\end{array}$ & 1996 & $\mathrm{Xu}$ & \begin{tabular}{|l} 
Chenggang \\
\end{tabular} & LSE & 0 & 1 & 2 & 0 & \\
\hline $\begin{array}{l}\text { Chinese Township-Village } \\
\text { Enterprises as Vaguely Defined } \\
\text { Cooperatives }\end{array}$ & $\begin{array}{l}\text { Weitzman,-Martin-L.; } \\
\text { Xu,-Chenggang }\end{array}$ & \begin{tabular}{|l|} 
Journal-of-Comparative \\
Economics; 18(2), April 1994, \\
pp. 121-45.
\end{tabular} & 1994 & $\mathrm{Xu}$ & Chenggang & LSE & 1.27 & 1 & 2 & 0.8466666667 & \\
\hline $\begin{array}{l}\text { Why China's Economic Reforms } \\
\text { Differ: The M-Form Hierarchy and } \\
\text { Entry/Expansion of the Non-state } \\
\text { Sector }\end{array}$ & $\begin{array}{l}\text { Qian,-Yingyi; Xu,-- } \\
\text { Chenggang }\end{array}$ & $\begin{array}{l}\text { Economics-of-Transition; 1(2), } \\
\text { June 1993, pp. 135-70. }\end{array}$ & 1993 & $\mathrm{Xu}$ & Chenggang & LSE & 0 & 1 & 2 & 0 & \\
\hline $\begin{array}{l}\text { The M-Form Hierarchy and China's } \\
\text { Economic Reform }\end{array}$ & $\begin{array}{l}\text { Qian,-Yingyi; Xu,-- } \\
\text { Chenggang }\end{array}$ & $\begin{array}{l}\text { European-Economic-Review; } \\
\text { 37(2-3), April 1993, pp. 541- } \\
48 .\end{array}$ & 1993 & $\mathrm{Xu}$ & Chenggang & LSE & 0.84 & 0.5 & 2 & 0.28 & C. Xu: 4.386666667 \\
\hline
\end{tabular}

Average: 2.953420139 
Rheinische Friedrich-Wilhelms-Universität Bonn

\begin{tabular}{|c|c|c|c|c|c|c|c|c|c|c|c|}
\hline & Author(s) & Journal & Year & & & & \begin{tabular}{|l|} 
Impact \\
Factor \\
\end{tabular} & \begin{tabular}{|c|} 
Seiten- \\
korr.
\end{tabular} & Authors & Impact & personal Impact \\
\hline $\begin{array}{l}\text { German Dominance in the EMS: } \\
\text { The Empirical Evidence }\end{array}$ & $\begin{array}{l}\text { Fratianni,-Michele; } \\
\text { von-Hagen,-Juergen }\end{array}$ & $\begin{array}{l}\text { Open-Economies- } \\
\text { Review; } 1(1), 1990, \\
\text { pp. } 67-87 .\end{array}$ & 1990 & Hagen & Jürgen von & $\begin{array}{l}\text { Rhein.- Fr.- } \\
\text { Wilhs.-U Bonn }\end{array}$ & 0 & 1 & 2 & 0 & \\
\hline $\begin{array}{l}\text { Public Choice Aspects of European } \\
\text { Monetary Unification }\end{array}$ & $\begin{array}{l}\text { Frantianni,-Michele; } \\
\text { von-Hagen,-Juergen }\end{array}$ & $\begin{array}{l}\text { Cato-Journal; } 10(2), \text { Fall } \\
\text { 1990, pp. 389-411. }\end{array}$ & 1990 & Hagen & Jürgen von & \begin{tabular}{|l} 
Rhein.- Fr.- \\
Wilhs-U Bonn
\end{tabular} & 0 & 1 & 2 & 0 & \\
\hline $\begin{array}{l}\text { Policy Effectiveness in an Open } \\
\text { Multi-market Economy with Risk } \\
\text { Neutral Exchange Rate Speculation }\end{array}$ & von-Hagen,-Juergen & $\begin{array}{l}\text { Journal-of-International- } \\
\text { Money-and-Finance; } \\
\text { 9(2), June 1990, } \\
\text { pp. 110-22. }\end{array}$ & 1990 & Hagen & Jürgen von & \begin{tabular}{|l|} 
Rhein.- Fr.- \\
Wilhs.-U Bonn
\end{tabular} & 0.49 & 1 & 1 & 0.49 & \\
\hline $\begin{array}{l}\text { The European Monetary System Ten } \\
\text { Years After }\end{array}$ & $\begin{array}{l}\text { Fratianni,-Michele; } \\
\text { von-Hagen,-Juergen }\end{array}$ & $\begin{array}{l}\text { Carnegie-Rochester- } \\
\text { Conference-Series-on- } \\
\text { Public-Policy; 32(0), } \\
\text { 1990, pp. 173-241. }\end{array}$ & 1990 & Hagen & Jürgen von & \begin{tabular}{|l|} 
Rhein.-Fr.- \\
Wilhs.-U. Bonn
\end{tabular} & 0 & 1 & 2 & 0 & J. v. Hagen: 0.49 \\
\hline $\begin{array}{l}\text { Demand Aggregation under } \\
\text { Structural Stability }\end{array}$ & $\begin{array}{l}\text { Hildenbrand,-Werner; } \\
\text { Kneip,-A. }\end{array}$ & $\begin{array}{l}\text { Journal-of-Mathematical- } \\
\text { Economics; 31(1), } \\
\text { February 1999, pp. } 81- \\
109 .\end{array}$ & 1999 & Hildenbrand & Werner & \begin{tabular}{|l|} 
Rhein.- Fr.- \\
Wilhs.-U Bonn
\end{tabular} & 0.37 & 1 & 2 & 0.246666667 & \\
\hline $\begin{array}{l}\text { Special Issue on Aggregation: } \\
\text { Editorial }\end{array}$ & Hildenbrand,-Werner & $\begin{array}{l}\text { Journal-of-Mathematical- } \\
\text { Economics; 31(1), } \\
\text { February } 1999 \text {, pp. 1-14. }\end{array}$ & 1999 & Hildenbrand & Werner & $\begin{array}{l}\text { Rhein.- Fr.- } \\
\text { Wilhs.-Uni. } \\
\text { Bonn }\end{array}$ & 0.37 & 1 & 1 & 0.37 & \\
\hline $\begin{array}{l}\text { How Relevant are Specifications of } \\
\text { Behavioral Relations on the Micro- } \\
\text { level for Modelling the Time Path of } \\
\text { Population Aggregates? }\end{array}$ & Hildenbrand,-Werner & $\begin{array}{l}\text { European-Economic- } \\
\text { Review; 42(3-5), May } \\
\text { 1998, pp. 437-58. }\end{array}$ & 1998 & Hildenbrand & Werner & \begin{tabular}{|l|} 
Rhein.- Fr.- \\
Wilhs.-U Bonn
\end{tabular} & 0.84 & 1 & 1 & 0.84 & \\
\hline $\begin{array}{l}\text { Family Expenditure Data, } \\
\text { Heteroscedasticity and the Law of } \\
\text { Demand }\end{array}$ & $\begin{array}{l}\text { Hildenbrand,-Werner; } \\
\text { Kneip,-Alois }\end{array}$ & $\begin{array}{l}\text { Ricerche-Economiche; } \\
47(2), \text { June } 1993, \\
\text { pp. 137-65. }\end{array}$ & 1993 & Hildenbrand & Werner & \begin{tabular}{|l|} 
Rhein.- Fr.- \\
Wilhs.-U Bonn
\end{tabular} & 0 & 1 & 2 & 0 & \\
\hline $\begin{array}{l}\text { Francis Ysidro Edgeworth: Perfect } \\
\text { Competition and the Core }\end{array}$ & Hildenbrand,-Werner & $\begin{array}{l}\text { European-Economic- } \\
\text { Review; 37(2-3), April } \\
\text { 1993, pp. 477-90. }\end{array}$ & 1993 & Hildenbrand & Werner & \begin{tabular}{|l|} 
Rhein.- Fr.- \\
Wilhs.-U Bonn
\end{tabular} & 0.84 & 1 & 1 & 0.84 & \\
\hline $\begin{array}{l}\text { Empirical Evidence on the Law of } \\
\text { Demand }\end{array}$ & $\begin{array}{l}\text { Hardle,-Wolfgang; } \\
\text { Hildenbrand,-Werner; } \\
\text { Jerison,-Michael } \\
\end{array}$ & \begin{tabular}{|l|} 
Econometrica; 59(6), \\
November 1991, \\
pp. 1525-49. \\
\end{tabular} & 1991 & Hildenbrand & Werner & \begin{tabular}{|l} 
Rhein.- Fr.- \\
Wilhs.-U Bonn
\end{tabular} & 2.22 & 1 & 3 & 1.11 & $\begin{array}{l}\text { W. Hildenbrand: } \\
3.406666667\end{array}$ \\
\hline $\begin{array}{l}\text { Abraham Wald's Equilibrium } \\
\text { Existence Proof Reconsidered }\end{array}$ & John,-Reinhard & $\begin{array}{l}\text { Economic-Theory; } 13(2), \\
\text { March } 1999, \text { pp. } 417-28 .\end{array}$ & 1999 & John & Reihnhard & \begin{tabular}{|l|} 
Rhein.- Fr.- \\
Wilhs.-U Bonn
\end{tabular} & 0 & 1 & 1 & 0 & \\
\hline $\begin{array}{l}\text { A Simple Cycle Preserving Extension } \\
\text { of a Demand Function }\end{array}$ & John,-Reinhard & $\begin{array}{l}\text { Journal-of-Economic- } \\
\text { Theory; } 72(2), \text { February } \\
\text { 1997, pp. 442-45. }\end{array}$ & 1997 & John & Reihnhard & \begin{tabular}{|l|} 
Rhein.- Fr.- \\
Wilhs.-U Bonn
\end{tabular} & 0.93 & 0.5 & 1 & 0.465 & \\
\hline $\begin{array}{l}\text { The Weak Axiom of Revealed } \\
\text { Preference and Homogeneity of } \\
\text { Demand Functions }\end{array}$ & John,-Reinhard & $\begin{array}{l}\text { Economics-Letters; } \\
47(1), \text { January } 1995 \\
\text { pp. 11-16. }\end{array}$ & 1995 & John & Reihnhard & \begin{tabular}{|l|} 
Rhein.- Fr.- \\
Wilhs.-U Bonn
\end{tabular} & 0.26 & 0.5 & 1 & 0.13 & R. John: 0.595 \\
\hline The Value of Ignorance & Kessler,-Anke-S. & $\begin{array}{l}\text { RAND-Journal-of- } \\
\text { Economics; 29(2), } \\
\text { Summer 1998, pp. 339- } \\
54 .\end{array}$ & 1998 & Kessler & Anke & \begin{tabular}{|l|} 
Rhein.- Fr.- \\
Wilhs.-U Bonn
\end{tabular} & 1.13 & 1 & 1 & 1.13 & A. Kessler: 1.13 \\
\hline
\end{tabular}


Rheinische Friedrich-Wilhelms-Universität Bonn

\begin{tabular}{|c|c|c|c|c|c|c|c|c|c|c|c|}
\hline & Author(s) & Journal & Year & & & & \begin{tabular}{|l|} 
Impact \\
Factor
\end{tabular} & \begin{tabular}{|c|} 
Seiten- \\
korr.
\end{tabular} & Authors & Impact & personal Impact \\
\hline $\begin{array}{l}\text { US Trade Balance Dynamics: The } \\
\text { Role of Fiscal Policy and Productivity } \\
\text { Shocks and of Financial Market } \\
\text { Linkages }\end{array}$ & Kollmann,-Robert & $\begin{array}{l}\text { Journal-of-International- } \\
\text { Money-and-Finance; } \\
\text { 17(4), August 1998, } \\
\text { pp. 637-69. }\end{array}$ & 1998 & Kollmann & Robert & $\begin{array}{l}\text { Rhein.- Fr.- } \\
\text { Wilhs.-U Bonn }\end{array}$ & 0.49 & 1 & 1 & 0.49 & \\
\hline $\begin{array}{l}\text { The Cyclical Behavior of Mark Ups in } \\
\text { U.S. Manufacturing and Trade: New } \\
\text { Empirical Evidence Based on a } \\
\text { Model of Optimal Storage }\end{array}$ & Kollmann,-Robert & $\begin{array}{l}\text { Economics-Letters; } \\
\text { 57(3), December 1997, } \\
\text { pp. 331-37. }\end{array}$ & 1997 & Kollmann & Robert & $\begin{array}{l}\text { Rhein.- Fr.- } \\
\text { Wilhs.-U Bonn }\end{array}$ & 0.26 & 0.5 & 1 & 0.13 & \\
\hline $\begin{array}{l}\text { Endogenous Fertility in a Model with } \\
\text { Non-dynastic Parental Altruism }\end{array}$ & Kollmann,-Robert & $\begin{array}{l}\text { Journal-of-Population- } \\
\text { Economics; 10(1), } \\
\text { February 1997, pp. 87- } \\
95 .\end{array}$ & 1997 & Kollmann & Robert & \begin{tabular}{|l} 
Rhein.- Fr.- \\
Wilhs.-U Bonn
\end{tabular} & 0.44 & 0.5 & 1 & 0.22 & \\
\hline $\begin{array}{l}\text { Incomplete Asset Markets and the } \\
\text { Cross-Country Consumption } \\
\text { Correlation Puzzle }\end{array}$ & Kollmann,-Robert & $\begin{array}{l}\text { Journal-of-Economic- } \\
\text { Dynamics-and-Control; } \\
\text { 20(5), May 1996, } \\
\text { pp. 945-61. }\end{array}$ & 1996 & Kollmann & Robert & $\begin{array}{l}\text { Rhein.- Fr.- } \\
\text { Wilhs.-U Bonn }\end{array}$ & 0.53 & 1 & 1 & 0.53 & \\
\hline $\begin{array}{l}\text { The Correlation of Productivity } \\
\text { Growth across Regions and } \\
\text { Industries in the United States }\end{array}$ & Kollmann,-Robert & $\begin{array}{l}\text { Economics-Letters; } \\
\text { 47(3-4), March 1995, } \\
\text { pp. 437-43. }\end{array}$ & 1995 & Kollmann & Robert & $\begin{array}{l}\text { Rhein.- Fr.- } \\
\text { Wilhs.-U Bonn }\end{array}$ & 0.26 & 0.5 & 1 & 0.13 & \\
\hline $\begin{array}{l}\text { Consumption, Real Exchange Rates } \\
\text { and the Structure of International } \\
\text { Asset Markets }\end{array}$ & Kollmann,-Robert & $\begin{array}{l}\text { Journal-of-International- } \\
\text { Money-and-Finance; } \\
\text { 14(2), April 1995, } \\
\text { pp. 191-211. }\end{array}$ & 1995 & Kollmann & Robert & $\begin{array}{l}\text { Rhein.- Fr.- } \\
\text { Wilhs.-U Bonn }\end{array}$ & 0.49 & 1 & 1 & 0.49 & \\
\hline $\begin{array}{l}\text { Hidden Unemployment: A Search- } \\
\text { Theoretic Interpretation }\end{array}$ & Kollmann,-Robert & $\begin{array}{l}\text { Economics-Letters; } \\
\text { 46(4), December 1994, } \\
\text { pp. 363-67. }\end{array}$ & 1994 & Kollmann & Robert & $\begin{array}{l}\text { Rhein.- Fr.- } \\
\text { Wilhs.-U Bonn }\end{array}$ & 0.26 & 0.5 & 1 & 0.13 & \\
\hline $\begin{array}{l}\text { The Duration of Unemployment as a } \\
\text { Signal }\end{array}$ & Kollmann,-Robert & $\begin{array}{l}\text { Economics-Letters; } \\
\text { 45(3), July 1994, } \\
\text { pp. 373-77. }\end{array}$ & 1994 & Kollmann & Robert & \begin{tabular}{|l|} 
Rhein.- Fr.- \\
Wilhs.-U Bonn
\end{tabular} & 0.26 & 0.5 & 1 & 0.13 & R. Kollmann: 2.25 \\
\hline $\begin{array}{l}\text { Auftragsterminierung fur die taktisch- } \\
\text { operative Personaleinsatzplanung in } \\
\text { Wirtschaftsprufungsgesellschaften. }\end{array}$ & \begin{tabular}{|l|} 
Salewski,-Frank; \\
Bartsch,-Thomas; \\
Pesch,-Erwin
\end{tabular} & $\begin{array}{l}\text { Zeitschrift-fur- } \\
\text { Betriebswirtschaft; 66(3), } \\
\text { March 1996, pp. 327-51. }\end{array}$ & 1996 & Pesch & Erwin & $\begin{array}{l}\text { Rhein.- Fr.- } \\
\text { Wilhs.-U Bonn }\end{array}$ & 0 & 1 & 3 & 0 & \\
\hline $\begin{array}{l}\text { Zur Bedeutung der Modellbildung fur } \\
\text { die Entwicklung wissensbasierter } \\
\text { Systeme. }\end{array}$ & $\begin{array}{l}\text { Drexl,-Andreas; } \\
\text { Pesch,-Erwin; } \\
\text { Salewski,-Frank }\end{array}$ & $\begin{array}{l}\text { Zeitschrift-fur- } \\
\text { Betriebswirtschaft; } \\
\text { 65(10), October 1995, } \\
\text { pp. 1135-53. }\end{array}$ & 1995 & Pesch & Erwin & $\begin{array}{l}\text { Rhein.- Fr.- } \\
\text { Wilhs.-U Bonn }\end{array}$ & 0 & 1 & 3 & 0 & E. Pesch: 0 \\
\hline $\begin{array}{l}\text { On the Multi-preference Approach to } \\
\text { Evaluating Opportunities }\end{array}$ & $\begin{array}{l}\text { Nehring,-Klaus; } \\
\text { Puppe,-Clemens }\end{array}$ & $\begin{array}{l}\text { Social-Choice-and- } \\
\text { Welfare; } 16(1) \text {, January } \\
\text { 1999, pp. } 41-63 .\end{array}$ & 1999 & Puppe & Clemens & $\begin{array}{l}\text { Rhein.- Fr.- } \\
\text { Wilhs.-U Bonn }\end{array}$ & 0.55 & 1 & 2 & 0.366666667 & \\
\hline $\begin{array}{l}\text { Voluntary Contributions When the } \\
\text { Public Good Is Not Necessarily } \\
\text { Normal }\end{array}$ & $\begin{array}{l}\text { Kerschbamer,-Rudolf; } \\
\text { Puppe,-Clemens }\end{array}$ & $\begin{array}{l}\text { Journal-of-Economics- } \\
\text { (Zeitschrift-fur- } \\
\text { Nationalokonomie); } \\
68(2), 1998 \text {, pp. 175-92. }\end{array}$ & 1998 & Puppe & Clemens & \begin{tabular}{|l|} 
Rhein.- Fr.- \\
Wilhs.-U Bonn
\end{tabular} & 0.18 & 1 & 2 & 0.12 & \\
\hline $\begin{array}{l}\text { On the Possibility of Efficient Private } \\
\text { Provision of Public Goods through } \\
\text { Government Subsidies }\end{array}$ & $\begin{array}{l}\text { Kirchsteiger,-Georg; } \\
\text { Puppe,-Clemens }\end{array}$ & $\begin{array}{l}\text { Journal-of-Public- } \\
\text { Economics; 66(3), } \\
\text { December 1997, } \\
\text { pp. 489-504. }\end{array}$ & 1997 & Puppe & Clemens & \begin{tabular}{|l|} 
Rhein.- Fr.- \\
Wilhs.-U Bonn
\end{tabular} & 0.82 & 1 & 2 & 0.546666667 & \\
\hline
\end{tabular}


Rheinische Friedrich-Wilhelms-Universität Bonn

\begin{tabular}{|c|c|c|c|c|c|c|c|c|c|c|c|}
\hline & Author(s) & Journal & Year & & & & \begin{tabular}{|l|} 
Impact \\
Factor
\end{tabular} & $\begin{array}{c}\text { Seiten- } \\
\text { korr. }\end{array}$ & Authors & Impact & personal Impact \\
\hline $\begin{array}{l}\text { On the Formation of Political } \\
\text { Coalitions }\end{array}$ & $\begin{array}{l}\text { Kirchsteiger,-Georg; } \\
\text { Puppe,-Clemens }\end{array}$ & $\begin{array}{l}\text { Journal-of-Institutional- } \\
\text { and-Theoretical- } \\
\text { Economics; 153(2), June } \\
\text { 1997, pp. 293-319. }\end{array}$ & 1997 & Puppe & Clemens & $\begin{array}{l}\text { Rhein.- Fr.- } \\
\text { Wilhs.-U Bonn }\end{array}$ & 0.29 & 1 & 2 & 0.193333333 & \\
\hline $\begin{array}{l}\text { Intransitive Choices Based on } \\
\text { Transitive Preferences: The Case of } \\
\text { Menu-Dependent Information }\end{array}$ & $\begin{array}{l}\text { Kirchsteiger,-Georg; } \\
\text { Puppe,-Clemens }\end{array}$ & $\begin{array}{l}\text { Theory-and-Decision; } \\
41(1) \text {, July 1996, pp. } 37- \\
58 .\end{array}$ & 1996 & Puppe & Clemens & $\begin{array}{l}\text { Rhein.- Fr.- } \\
\text { Wilhs.-U Bonn }\end{array}$ & 0.56 & 1 & 2 & 0.373333333 & \\
\hline $\begin{array}{l}\text { Continuous Extensions of an Order } \\
\text { on a Set to the Power Set }\end{array}$ & $\begin{array}{l}\text { Nehring,-Klaus; } \\
\text { Puppe,-Clemens }\end{array}$ & $\begin{array}{l}\text { Journal-of-Economic- } \\
\text { Theory; 68(2), February } \\
\text { 1996, pp. 456-79. }\end{array}$ & 1996 & Puppe & Clemens & $\begin{array}{l}\text { Rhein.- Fr.- } \\
\text { Wilhs.-U Bonn }\end{array}$ & 0.93 & 1 & 2 & 0.62 & \\
\hline $\begin{array}{l}\text { An Axiomatic Approach to } \\
\text { "Preference for Freedom of Choice." }\end{array}$ & Puppe,-Clemens & $\begin{array}{l}\text { Journal-of-Economic- } \\
\text { Theory; 68(1), January } \\
\text { 1996, pp. 174-99. }\end{array}$ & 1996 & Puppe & Clemens & $\begin{array}{l}\text { Rhein.- Fr.- } \\
\text { Wilhs.-U Bonn }\end{array}$ & 0.93 & 1 & 1 & 0.93 & \\
\hline $\begin{array}{l}\text { Freedom of Choice and Rational } \\
\text { Decisions }\end{array}$ & Puppe,-Clemens & $\begin{array}{l}\text { Social-Choice-and- } \\
\text { Welfare; } 12(2), \text { March } \\
\text { 1995, pp. 137-53. } \\
\end{array}$ & 1995 & Puppe & Clemens & $\begin{array}{l}\text { Rhein.- Fr.- } \\
\text { Wilhs.-U Bonn }\end{array}$ & 0.55 & 1 & 1 & 0.55 & \\
\hline $\begin{array}{l}\text { Preference Functionals with Prize- } \\
\text { Dependent Distortion of Probabilities }\end{array}$ & Puppe,-Clemens & $\begin{array}{l}\text { Economics-Letters; } \\
\text { 33(2), June 1990, } \\
\text { pp. 127-31. }\end{array}$ & 1990 & Puppe & Clemens & $\begin{array}{l}\text { Rhein.- Fr.- } \\
\text { Wilhs.-U Bonn }\end{array}$ & 0.26 & 0.5 & 1 & 0.13 & C. Puppe: 3.83 \\
\hline $\begin{array}{l}\text { Know-how Disclosure and } \\
\text { Incomplete Contracts }\end{array}$ & $\begin{array}{l}\text { Rosenkranz,-- } \\
\text { Stephanie; Schmitz,- } \\
\text { Patrick-W. }\end{array}$ & $\begin{array}{l}\text { Economics-Letters; } \\
\text { 63(2), May 1999, } \\
\text { pp. 181-85. }\end{array}$ & 1999 & Rosenkranz & Stephanie & \begin{tabular}{|l|} 
Rhein.- Fr.- \\
Wilhs.-U Bonn
\end{tabular} & 0.26 & 0.5 & 2 & 0.086666667 & \\
\hline $\begin{array}{l}\text { Quality Improvements and the } \\
\text { Incentive to Leapfrog }\end{array}$ & $\begin{array}{l}\text { Rosenkranz,- } \\
\text { Stephanie }\end{array}$ & $\begin{array}{l}\text { International-Journal-of- } \\
\text { Industrial-Organization; } \\
\text { 15(2), April 1997, } \\
\text { pp. 243-61. } \\
\end{array}$ & 1997 & Rosenkranz & Stephanie & $\begin{array}{l}\text { Rhein.- Fr.- } \\
\text { Wilhs.-U Bonn }\end{array}$ & 0.34 & 1 & 1 & 0.34 & \\
\hline $\begin{array}{l}\text { Innovation and Cooperation under } \\
\text { Vertical Product Differentiation }\end{array}$ & $\begin{array}{l}\text { Rosenkranz,- } \\
\text { Stephanie }\end{array}$ & \begin{tabular}{|l} 
International-Journal-of- \\
Industrial-Organization; \\
13(1), 1995, pp. 1-22. \\
\end{tabular} & 1995 & Rosenkranz & Stephanie & \begin{tabular}{|l|} 
Rhein.- Fr.- \\
Wilhs.-U Bonn
\end{tabular} & 0.34 & 1 & 1 & 0.34 & $\begin{array}{l}\text { S. Rosenkranz } \\
0.766666667\end{array}$ \\
\hline Hermann Sabel zum 60. Geburtstag & Kloock,-Josef & $\begin{array}{l}\text { Zeitschrift-fur- } \\
\text { Betriebswirtschaft; 67(2), } \\
\text { February 1997, pp. 121- } \\
22 .\end{array}$ & 1997 & Sabel & Hermann & $\begin{array}{l}\text { Rhein.- Fr.- } \\
\text { Wilhs.-U Bonn }\end{array}$ & 0 & 0.5 & 1 & 0 & \\
\hline $\begin{array}{l}\text { In eigener Sache. Dem Schriftleiter } \\
\text { der ZfB, horst Albach, zum } \\
\text { Funfundsechzigsten }\end{array}$ & Sabel,-Hermann & \begin{tabular}{|l|} 
Zeitschrift-fur- \\
Betriebswirtschaft; 66(7), \\
July 1996, pp. 777-90. \\
\end{tabular} & 1996 & Sabel & Hermann & $\begin{array}{l}\text { Rhein.- Fr.- } \\
\text { Wilhs.-U Bonn }\end{array}$ & 0 & 1 & 1 & 0 & \\
\hline Zum Sterben von Unternehmen. & $\begin{array}{l}\text { Sabel,-Hermann; } \\
\text { Weiser,-Christoph }\end{array}$ & $\begin{array}{l}\text { Zeitschrift-fur- } \\
\text { Betriebswirtschaft; 64(3), } \\
\text { March 1994, pp. 297- } \\
\text { 312. } \\
\end{array}$ & 1994 & Sabel & Hermann & \begin{tabular}{|l|} 
Rhein.- Fr.- \\
Wilhs.-U Bonn
\end{tabular} & 0 & 1 & 2 & 0 & \\
\hline $\begin{array}{l}\text { Economies und Savings als } \\
\text { grundlegende Konzepte der } \\
\text { Erfahrung. Was bringt mehr? }\end{array}$ & $\begin{array}{l}\text { Klook,-Josef; Sabel,-- } \\
\text { Hermann }\end{array}$ & \begin{tabular}{|l|} 
Zeitschrift-fur- \\
Betriebsw irtschaft; 63(3), \\
March 1993, pp. 209-33. \\
\end{tabular} & 1993 & Sabel & Hermann & $\begin{array}{l}\text { Rhein.- Fr.- } \\
\text { Wilhs.-U Bonn }\end{array}$ & 0 & 1 & 2 & 0 & : \\
\hline $\begin{array}{l}\text { Qualitaten, Preise und Mengen. } \\
\text { Befunde auf dem Markt der } \\
\text { Personenkraftwagen der } \\
\text { Bundesrepublik Deutschland. }\end{array}$ & Sabel,-Hermann & $\begin{array}{l}\text { Zeitschrift-fur- } \\
\text { Betriebswirtschaft; } 60(8), \\
\text { August 1990, pp. 745- } \\
72 . \\
\end{array}$ & 1990 & Sabel & Hermann & \begin{tabular}{|l|} 
Rhein.- Fr.- \\
Wilhs.-U Bonn
\end{tabular} & 0 & 1 & 1 & 0 & H. Sabel 0 \\
\hline
\end{tabular}


Rheinische Friedrich-Wilhelms-Universität Bonn

\begin{tabular}{|c|c|c|c|c|c|c|c|c|c|c|c|}
\hline & Author(s) & Journal & Year & & & & \begin{tabular}{|l|} 
Impact \\
Factor
\end{tabular} & \begin{tabular}{|c|} 
Seiten- \\
korr.
\end{tabular} & Authors & Impact & personal Impact \\
\hline $\begin{array}{l}\text { Monopolistic Provision of Excludable } \\
\text { Public Goods under Private } \\
\text { Information }\end{array}$ & Schmitz,-Patrick-W. & $\begin{array}{l}\text { Public-Finance; 52(1), } \\
\text { 1997, pp. 89-101. }\end{array}$ & 1997 & Schmitz & Patrick & $\begin{array}{l}\text { Rhein.- Fr.- } \\
\text { Wilhs.-U Bonn }\end{array}$ & 0 & 1 & 1 & 0 & \\
\hline $\begin{array}{l}\text { Know-how Disclosure and } \\
\text { Incomplete Contracts }\end{array}$ & $\begin{array}{l}\text { Rosenkranz,- } \\
\text { Stephanie; Schmitz,- } \\
\text { Patrick-W. }\end{array}$ & $\begin{array}{l}\text { Economics-Letters; } \\
\text { 63(2), May 1999, } \\
\text { pp. 181-85. }\end{array}$ & 1999 & Schmitz & Patrick & $\begin{array}{l}\text { Rhein.- Fr.- } \\
\text { Wilhs.-U Bonn }\end{array}$ & 0.26 & 0.5 & 2 & 0.086666667 & \\
\hline $\begin{array}{l}\text { Ex Post Liability for Harm vs. Ex Ante } \\
\text { Safety Regulation: Substitutes or } \\
\text { Complements? Comment }\end{array}$ & $\begin{array}{l}\text { Ewerhart,-Christian; } \\
\text { Schmitz,-Patrick-W. }\end{array}$ & $\begin{array}{l}\text { American-Economic- } \\
\text { Review; 88(4), } \\
\text { September 1998, } \\
\text { pp. } 1027 .\end{array}$ & 1998 & Schmitz & Patrick & \begin{tabular}{|l|} 
Rhein.- Fr.- \\
Wilhs.-U Bonn
\end{tabular} & 1.74 & 1 & 2 & 1.16 & \begin{tabular}{|l|} 
P. Schmitz: \\
1.676666667
\end{tabular} \\
\hline Randomization in Coalition Contracts & Schmitz,-Patrick-W. & $\begin{array}{l}\text { Public-Choice; 94(3-4), } \\
\text { March 1998, pp. 341-53. }\end{array}$ & 1998 & Schmitz & Patrick & \begin{tabular}{|l|} 
Rhein.- Fr.- \\
Wilhs.-U Bonn
\end{tabular} & 0.43 & 1 & 1 & 0.43 & \\
\hline $\begin{array}{l}\text { Proper Rationalizability and } \\
\text { Backward Induction }\end{array}$ & Schuhmacher,-Frank & $\begin{array}{l}\text { International-Journal-of- } \\
\text { Game-Theory; 28(4), } \\
\text { November 1999, } \\
\text { pp. 599-615. }\end{array}$ & 1999 & Schuhmacher & Frank & \begin{tabular}{|l|} 
Rhein.- Fr.- \\
Wilhs.-U Bonn
\end{tabular} & 0.31 & 1 & 1 & 0.31 & $\begin{array}{l}\text { F. Schuhmacher: } \\
0.31\end{array}$ \\
\hline $\begin{array}{l}\text { Lotteries: Determinants of Ticket } \\
\text { Sales and the Optimal Payout Rate: } \\
\text { Discussion }\end{array}$ & Schw eizer,-Urs & $\begin{array}{l}\text { Economic-Policy:-A- } \\
\text { European-Forum; 0(27), } \\
\text { October 1998, pp. 395- } \\
98 .\end{array}$ & 1998 & Schweizer & Urs & $\begin{array}{l}\text { Rhein.- Fr.- } \\
\text { Wilhs.-U Bonn }\end{array}$ & 0 & 0.5 & 1 & 0 & \\
\hline $\begin{array}{l}\text { Endogenous Fertility and the Henry } \\
\text { George Theorem }\end{array}$ & Schweizer,-Urs & $\begin{array}{l}\text { Journal-of-Public- } \\
\text { Economics; 61(2), } \\
\text { August 1996, pp. 209- } \\
28 .\end{array}$ & 1996 & Schweizer & Urs & \begin{tabular}{|l|} 
Rhein.- Fr.- \\
Wilhs.-U Bonn
\end{tabular} & 0.82 & 1 & 1 & 0.82 & \\
\hline $\begin{array}{l}\text { The Phenomenology of Contract: } \\
\text { Complex Contracting in the } \\
\text { Entertainment Industry: Comment }\end{array}$ & Schweizer,-Urs & $\begin{array}{l}\text { Journal-of-Institutional- } \\
\text { and-Theoretical- } \\
\text { Economics; 152(1), } \\
\text { March 1996, pp. 140-45. }\end{array}$ & 1996 & Schweizer & Urs & $\begin{array}{l}\text { Rhein.- Fr.- } \\
\text { Wilhs.-U Bonn }\end{array}$ & 0.29 & 0.5 & 1 & 0.145 & \\
\hline $\begin{array}{l}\text { Binmore's and Samuelson's } \\
\text { Perspective on the Evolution of } \\
\text { Norms: Comment }\end{array}$ & Schweizer,-Urs & $\begin{array}{l}\text { Journal-of-Institutional- } \\
\text { and-Theoretical- } \\
\text { Economics; 150(1), } \\
\text { March 1994, pp. 64-67. }\end{array}$ & 1994 & Schweizer & Urs & \begin{tabular}{|l|} 
Rhein.- Fr.- \\
Wilhs.-U Bonn
\end{tabular} & 0.29 & 0.5 & 1 & 0.145 & \\
\hline $\begin{array}{l}\text { Institutional Choice: A Contract- } \\
\text { Theoretic Approach }\end{array}$ & Schweizer,-Urs & $\begin{array}{l}\text { Journal-of-Institutional- } \\
\text { and-Theoretical- } \\
\text { Economics; 149(1), } \\
\text { March 1993, pp. 151-69. }\end{array}$ & 1993 & Schweizer & Urs & $\begin{array}{l}\text { Rhein.- Fr.- } \\
\text { Wilhs.-U Bonn }\end{array}$ & 0.29 & 1 & 1 & 0.29 & \\
\hline $\begin{array}{l}\text { The "Foreclosure" Effects of Vertical } \\
\text { Mergers: Comment }\end{array}$ & Schweizer,-Urs & $\begin{array}{l}\text { Journal-of-Institutional- } \\
\text { and-Theoretical- } \\
\text { Economics; 147(1), } \\
\text { March 1991, pp. 227-31. }\end{array}$ & 1991 & Schweizer & Urs & \begin{tabular}{|l|} 
Rhein.- Fr.- \\
Wilhs.-U Bonn
\end{tabular} & 0.29 & 0.5 & 1 & 0.145 & \\
\hline $\begin{array}{l}\text { Calculus of Consent: A Game- } \\
\text { Theoretic Perspective }\end{array}$ & Schweizer,-Urs & $\begin{array}{l}\text { Journal-of-Institutional- } \\
\text { and-Theoretical- } \\
\text { Economics; 146(1), } \\
\text { March 1990, pp. 28-54. }\end{array}$ & 1990 & Schweizer & Urs & $\begin{array}{l}\text { Rhein.- Fr.- } \\
\text { Wilhs.-U Bonn }\end{array}$ & 0.29 & 1 & 1 & 0.29 & $\begin{array}{l}\text { U. Schweizer: } \\
1.835\end{array}$ \\
\hline $\begin{array}{l}\text { Money Does Not Induce Risk Neutral } \\
\text { Behavior, but Binary Lotteries Do } \\
\text { Even Worse }\end{array}$ & $\begin{array}{l}\text { Selten,-Reinhard; } \\
\text { Sadrieh,-Abdolkarim; } \\
\text { Abbink,-Klaus }\end{array}$ & $\begin{array}{l}\text { Theory-and-Decision; } \\
\text { 46(3), June 1999, } \\
\text { pp. 211-49. }\end{array}$ & 1999 & Selten & Reinhard & $\begin{array}{l}\text { Rhein.- Fr.- } \\
\text { Wilhs.-U Bonn }\end{array}$ & 0.56 & 1 & 3 & 0.28 & \\
\hline
\end{tabular}


Rheinische Friedrich-Wilhelms-Universität Bonn

\begin{tabular}{|c|c|c|c|c|c|c|c|c|c|c|c|}
\hline & Author(s) & Journal & Year & & & & \begin{tabular}{|l|} 
Impact \\
Factor \\
\end{tabular} & \begin{tabular}{|c|}
$\begin{array}{c}\text { Seiten- } \\
\text { korr. }\end{array}$ \\
\end{tabular} & Authors & Impact & personal Impact \\
\hline $\begin{array}{l}\text { Axiomatic Characterization of the } \\
\text { Quadratic Scoring Rules }\end{array}$ & Selten,-Reinhard & $\begin{array}{l}\text { Experimental- } \\
\text { Economics; 1(1), 1998, } \\
\text { pp. 43-62. }\end{array}$ & 1998 & Selten & Reinhard & $\begin{array}{l}\text { Rhein.- Fr.- } \\
\text { Wilhs.-U Bonn }\end{array}$ & 0 & 1 & 1 & 0 & \\
\hline $\begin{array}{l}\text { Features of Experimentally Observed } \\
\text { Bounded Rationality }\end{array}$ & Selten,-Reinhard & $\begin{array}{l}\text { European-Economic- } \\
\text { Review; 42(3-5), May } \\
\text { 1998, pp. 413-36. }\end{array}$ & 1998 & Selten & Reinhard & $\begin{array}{l}\text { Rhein.- Fr.- } \\
\text { Wilhs.-U Bonn }\end{array}$ & 0.84 & 1 & 1 & 0.84 & \\
\hline $\begin{array}{l}\text { Multistage Game Models and Delay } \\
\text { Supergames }\end{array}$ & Selten,-Reinhard & $\begin{array}{l}\text { Theory-and-Decision; } \\
\text { 44(1), January 1998, } \\
\text { pp. 1-36. } \\
\end{array}$ & 1998 & Selten & Reinhard & \begin{tabular}{|l|} 
Rhein.- Fr.- \\
Wilhs.-U Bonn
\end{tabular} & 0.56 & 1 & 1 & 0.56 & \\
\hline An Experimental Solidarity Game & $\begin{array}{l}\text { Selten,--Reinhard; } \\
\text { Ockenfels,-Axel }\end{array}$ & $\begin{array}{l}\text { Journal-of-Economic- } \\
\text { Behavior-and- } \\
\text { Organization; 34(4), } \\
\text { March 1998, pp. 517-39. }\end{array}$ & 1998 & Selten & Reinhard & $\begin{array}{l}\text { Rhein.- Fr.- } \\
\text { Wilhs.-U Bonn }\end{array}$ & 0.42 & 1 & 2 & 0.28 & \\
\hline $\begin{array}{l}\text { Duopoly Strategies Programmed by } \\
\text { Experienced Players }\end{array}$ & $\begin{array}{l}\text { Selten,-Reinhard; } \\
\text { Mitzkewitz,-Michael; } \\
\text { Uhlich,-Gerald-R. }\end{array}$ & \begin{tabular}{|l|} 
Econometrica; 65(3), \\
May 1997, pp. 517-55.
\end{tabular} & 1997 & Selten & Reinhard & $\begin{array}{l}\text { Rhein.- Fr.- } \\
\text { Wilhs.-U Bonn }\end{array}$ & 2.22 & 1 & 3 & 1.11 & \\
\hline $\begin{array}{l}\text { An Axiomatic Theory of a Risk } \\
\text { Dominance Measure for Bipolar } \\
\text { Games with Linear Incentives }\end{array}$ & Selten,-Reinhard & $\begin{array}{l}\text { Games-and-Economic- } \\
\text { Behavior; 8(1), January } \\
\text { 1995, pp. 213-63. } \\
\end{array}$ & 1995 & Selten & Reinhard & $\begin{array}{l}\text { Rhein.- Fr.- } \\
\text { Wilhs.-U Bonn }\end{array}$ & 0.6 & 1 & 1 & 0.6 & \\
\hline $\begin{array}{l}\text { An Axiomatic Approach to } \\
\text { Consumers' Welfare }\end{array}$ & $\begin{array}{l}\text { Selten,-Reinhard; } \\
\text { Winter,-Eyal }\end{array}$ & $\begin{array}{l}\text { Mathematical-Social- } \\
\text { Sciences; } 27(1), \\
\text { February 1994, pp. 19- } \\
30 .\end{array}$ & 1994 & Selten & Reinhard & \begin{tabular}{|l} 
Rhein.- Fr.- \\
Wilhs.-U Bonn
\end{tabular} & 0.34 & 1 & 2 & 0.226666667 & \\
\hline $\begin{array}{l}\text { New Challenges to the Rationality } \\
\text { Assumption: Comment }\end{array}$ & Selten,-Reinhard & $\begin{array}{l}\text { Journal-of-Institutional- } \\
\text { and-Theoretical- } \\
\text { Economics; 150(1), } \\
\text { March 1994, pp. 42-44. }\end{array}$ & 1994 & Selten & Reinhard & $\begin{array}{l}\text { Rhein.- Fr.- } \\
\text { Wilhs.-U Bonn }\end{array}$ & 0.29 & 0.5 & 1 & 0.145 & \\
\hline $\begin{array}{l}\text { Demand Commitment Bargaining in } \\
\text { Three-Person Quota Game } \\
\text { Experiments }\end{array}$ & $\begin{array}{l}\text { Selten,-Reinhard; } \\
\text { Kuon,-Bettina }\end{array}$ & $\begin{array}{l}\text { International-Journal-of- } \\
\text { Game-Theory; 22(3), } \\
\text { 1993, pp. 261-77. } \\
\end{array}$ & 1993 & Selten & Reinhard & $\begin{array}{l}\text { Rhein.- Fr.- } \\
\text { Wilhs.-U Bonn }\end{array}$ & 0.31 & 1 & 2 & 0.206666667 & \\
\hline $\begin{array}{l}\text { Properties of a Measure of Predictive } \\
\text { Success }\end{array}$ & Selten,-Reinhard & $\begin{array}{l}\text { Mathematical-Social- } \\
\text { Sciences; } 21(2), \text { April } \\
\text { 1991, pp. 153-67. }\end{array}$ & 1991 & Selten & Reinhard & $\begin{array}{l}\text { Rhein.- Fr.- } \\
\text { Wilhs.-U Bonn }\end{array}$ & 0.34 & 1 & 1 & 0.34 & \\
\hline $\begin{array}{l}\text { Evolution, Learning, and Economic } \\
\text { Behavior }\end{array}$ & Selten,-Reinhard & $\begin{array}{l}\text { Games-and-Economic- } \\
\text { Behavior; 3(1), February } \\
\text { 1991, pp. 3-24. }\end{array}$ & 1991 & Selten & Reinhard & $\begin{array}{l}\text { Rhein.- Fr.- } \\
\text { Wilhs.-U Bonn }\end{array}$ & 0.6 & 1 & 1 & 0.6 & \\
\hline Bounded Rationality & Selten,-Reinhard & $\begin{array}{l}\text { Journal-of-Institutional- } \\
\text { and-Theoretical- } \\
\text { Economics; } 146(4) \text {, Dec. } \\
\text { 1990, pp. } 649-58 .\end{array}$ & 1990 & Selten & Reinhard & $\begin{array}{l}\text { Rhein.- Fr.- } \\
\text { Wilhs.-U Bonn }\end{array}$ & 0.29 & 0.5 & 1 & 0.145 & \\
\hline $\begin{array}{l}\text { Alternating Bid Bargaining with a } \\
\text { Smallest Money Unit }\end{array}$ & $\begin{array}{l}\text { van-Damme,-Eric; } \\
\text { Selten,-Reinhard; } \\
\text { Winter,-Eyal }\end{array}$ & $\begin{array}{l}\text { Games-and-Economic- } \\
\text { Behavior; 2(2), June } \\
\text { 1990, pp. 188-201. }\end{array}$ & 1990 & Selten & Reinhard & $\begin{array}{l}\text { Rhein.- Fr.- } \\
\text { Wilhs.-U Bonn }\end{array}$ & 0.6 & 1 & 3 & 0.3 & $\begin{array}{l}\text { R. Selten: } \\
5.633333333\end{array}$ \\
\hline $\begin{array}{l}\text { The Emergence of Kinship Behavior } \\
\text { in Structured Populations of } \\
\text { Unrelated Individuals }\end{array}$ & $\begin{array}{l}\text { Eshel,--Ilan; Sansone,-- } \\
\text { Emilia; Shaked,-Avner }\end{array}$ & $\begin{array}{l}\text { International-Journal-of- } \\
\text { Game-Theory; } 28(4), \\
\text { November 1999, } \\
\text { pp. 447-63. }\end{array}$ & 1999 & Shaked & Avner & $\begin{array}{l}\text { Rhein.- Fr.- } \\
\text { Wilhs.-U Bonn }\end{array}$ & 0.31 & 1 & 3 & 0.155 & \\
\hline
\end{tabular}


Rheinische Friedrich-Wilhelms-Universität Bonn

\begin{tabular}{|c|c|c|c|c|c|c|c|c|c|c|c|}
\hline & Author(s) & Journal & Year & & & & \begin{tabular}{|l|} 
Impact \\
Factor \\
\end{tabular} & \begin{tabular}{|c|}
$\begin{array}{c}\text { Seiten- } \\
\text { korr. }\end{array}$ \\
\end{tabular} & Authors & Impact & personal Impact \\
\hline $\begin{array}{l}\text { Altruists, Egoists, and Hooligans in a } \\
\text { Local Interaction Model }\end{array}$ & $\begin{array}{l}\text { Eshel,-Ilan; } \\
\text { Samuelson,-Larry; } \\
\text { Shaked,-Avner }\end{array}$ & $\begin{array}{l}\text { American-Economic- } \\
\text { Review; 88(1), March } \\
\text { 1998, pp. 157-79. }\end{array}$ & 1998 & Shaked & Avner & $\begin{array}{l}\text { Rhein.- Fr.- } \\
\text { Wilhs.-U Bonn }\end{array}$ & 1.74 & 1 & 3 & 0.87 & \\
\hline $\begin{array}{l}\text { Correlated Equilibria and Local } \\
\text { Interactions }\end{array}$ & $\begin{array}{l}\text { Mailath,-George-J.; } \\
\text { Samuelson,-Larry; } \\
\text { Shaked,-Avner }\end{array}$ & $\begin{array}{l}\text { Economic-Theory; 9(3), } \\
\text { 1997, pp. 551-56. }\end{array}$ & 1997 & Shaked & Avner & $\begin{array}{l}\text { Rhein.- Fr.- } \\
\text { Wilhs.-U Bonn }\end{array}$ & 0 & 0.5 & 3 & 0 & \\
\hline $\begin{array}{l}\text { Competition in Markets for Credence } \\
\text { Goods: Comment }\end{array}$ & Shaked,-Avner & $\begin{array}{l}\text { Journal-of-Institutional- } \\
\text { and-Theoretical- } \\
\text { Economics; 151(1), } \\
\text { March 1995, pp. 137-38. }\end{array}$ & 1995 & Shaked & Avner & $\begin{array}{l}\text { Rhein.- Fr.- } \\
\text { Wilhs.-U Bonn }\end{array}$ & 0.29 & 0.5 & 1 & 0.145 & \\
\hline $\begin{array}{l}\text { Opting Out: Bazaars versus 'Hi } \\
\text { Tech' Markets }\end{array}$ & Shaked,-Avner & $\begin{array}{l}\text { Investigaciones- } \\
\text { Economicas; 18(3), } \\
\text { September 1994, } \\
\text { pp. 421-32. }\end{array}$ & 1994 & Shaked & Avner & $\begin{array}{l}\text { Rhein.- Fr.- } \\
\text { Wilhs.-U Bonn }\end{array}$ & 0 & 1 & 1 & 0 & \\
\hline $\begin{array}{l}\text { Multiproduct Firms and Market } \\
\text { Structure }\end{array}$ & $\begin{array}{l}\text { Shaked,-Avner; } \\
\text { Sutton,-John }\end{array}$ & $\begin{array}{l}\text { Rand-Journal-of- } \\
\text { Economics; 21(1), } \\
\text { Spring 1990, pp. 45-62. }\end{array}$ & 1990 & Shaked & Avner & \begin{tabular}{|l|} 
Rhein.- Fr.- \\
Wilhs.-U Bonn
\end{tabular} & 1.13 & 1 & 2 & 0.753333333 & $\begin{array}{l}\text { A. Shaked: } \\
1.923333333\end{array}$ \\
\hline $\begin{array}{l}\text { A Note on the Stability of Lognormal } \\
\text { Interest Rate Models and the Pricing } \\
\text { of Eurodollar Futures }\end{array}$ & $\begin{array}{l}\text { Sandmann,-Klaus; } \\
\text { Sondermann,-Dieter }\end{array}$ & $\begin{array}{l}\text { Mathematical-Finance; } \\
\text { 7(2), April 1997, pp. 119- } \\
25 .\end{array}$ & 1997 & Sondermann & Dieter & $\begin{array}{l}\text { Rhein.- Fr.- } \\
\text { Wilhs.-U Bonn }\end{array}$ & 0 & 0.5 & 2 & 0 & \\
\hline $\begin{array}{l}\text { Closed Form Solutions for Term } \\
\text { Structure Derivatives with Log- } \\
\text { Normal Interest Rates }\end{array}$ & $\begin{array}{l}\text { Miltersen,-K.-R.; } \\
\text { Sandmann,-K.; } \\
\text { Sondermann,-D. }\end{array}$ & $\begin{array}{l}\text { Journal-of-Finance; } \\
\text { 52(1), March 1997, } \\
\text { pages 409-30. }\end{array}$ & 1997 & Sondermann & Dieter & \begin{tabular}{|l} 
Rhein.- Fr.- \\
Wilhs.-U Bonn
\end{tabular} & 2.12 & 1 & 3 & 1.06 & \\
\hline Zur Bewertung von Caps und Floors. & $\begin{array}{l}\text { Sondermann,-Dieter; } \\
\text { Sandmann,-Klaus }\end{array}$ & $\begin{array}{l}\text { Zeitschrift-fur- } \\
\text { Betriebswirtschaft; } \\
60(11), \text { November 1990, } \\
\text { pp. 1205-38. }\end{array}$ & 1990 & Sondermann & Dieter & $\begin{array}{l}\text { Rhein.- Fr.- } \\
\text { Wilhs.-U Bonn }\end{array}$ & 0 & 1 & 2 & 0 & $\begin{array}{l}\text { D. Sondermann: } \\
1.06\end{array}$ \\
\hline $\begin{array}{l}\text { The Index of Sustainable Economic } \\
\text { Welfare (ISEW) as an Alternative to } \\
\text { GDP in Measuring Economic } \\
\text { Welfare. }\end{array}$ & $\begin{array}{l}\text { Stockhammer,- } \\
\text { Engelbert et-al. }\end{array}$ & $\begin{array}{l}\text { Ecological-Economics; } \\
\text { 21(1), April 1997, pp. 19- } \\
\text { 34. }\end{array}$ & 1997 & Steiner & Klaus & \begin{tabular}{|l|} 
Rhein.- Fr.- \\
Wilhs.-U Bonn
\end{tabular} & 0.64 & 1 & 3 & 0.32 & K. Steiner: 0,32 \\
\hline $\begin{array}{l}\text { Liquiditatsmessung auf } \\
\text { experimentellen Aktienmarkten. }\end{array}$ & Theissen,-Erik & \begin{tabular}{|l|} 
Kredit-und-Kapital; \\
32(2), 1999, pp. 225-64.
\end{tabular} & 1999 & Theissen & Erik & \begin{tabular}{|l|} 
Rhein.- Fr.- \\
Wilhs.-U Bonn
\end{tabular} & 0 & 1 & 1 & 0 & \\
\hline $\begin{array}{l}\text { Der Neue Markt: Eine } \\
\text { Bestandsaufnahme. }\end{array}$ & Theissen,-Erik & $\begin{array}{l}\text { Zeitschrift-fur- } \\
\text { Wirtschafts--und- } \\
\text { Sozialwissenschaften; } \\
\text { 118(4), 1998, pp. 623- } \\
52 .\end{array}$ & 1998 & Theissen & Erik & \begin{tabular}{|l|} 
Rhein.- Fr.- \\
Wilhs.-U Bonn
\end{tabular} & 0 & 1 & 1 & 0 & \\
\hline $\begin{array}{l}\text { Inferring Risk Attitudes from Certainty } \\
\text { Equivalents: Some Lessons from an } \\
\text { Experimental Study }\end{array}$ & $\begin{array}{l}\text { Krahnen,-Jan-Pieter; } \\
\text { Rieck,-Christian; } \\
\text { Theissen,-Erik }\end{array}$ & $\begin{array}{l}\text { Journal-of-Economic- } \\
\text { Psychology; 18(5), } \\
\text { September 1997, } \\
\text { pp. 469-86. } \\
\end{array}$ & 1997 & Theissen & Erik & $\begin{array}{l}\text { Rhein.- Fr.- } \\
\text { Wilhs.-U Bonn }\end{array}$ & 0.24 & 1 & 3 & 0.12 & E. Theissen: 0.12 \\
\hline $\begin{array}{l}\text { Economic Incentives and } \\
\text { Hospitalization in Germany }\end{array}$ & Geil,-Peter et-al. & $\begin{array}{l}\text { Journal-of-Applied- } \\
\text { Econometrics; 12(3), } \\
\text { May-June 1997, pp. 295- } \\
311 .\end{array}$ & 1997 & Zimmermann & Klaus F. & \begin{tabular}{|l|} 
Rhein.- Fr.- \\
Wilhs.-U Bonn
\end{tabular} & 0.72 & 1 & 3 & 0.36 & \\
\hline
\end{tabular}


Rheinische Friedrich-Wilhelms-Universität Bonn

\begin{tabular}{|c|c|c|c|c|c|c|c|c|c|c|c|}
\hline & Author(s) & Journal & Year & & & & \begin{tabular}{|l|} 
Impact \\
Factor
\end{tabular} & $\begin{array}{c}\begin{array}{c}\text { Seiten- } \\
\text { korr. }\end{array} \\
\end{array}$ & Authors & Impact & personal Impact \\
\hline European Migration: Push and Pull & Zimmermann,-Klaus-F. & $\begin{array}{l}\text { International-Regional- } \\
\text { Science-Review; 19(1- } \\
\text { 2), 1996, pp. 95-128. }\end{array}$ & 1996 & Zimmermann & Klaus F. & $\begin{array}{l}\text { Rhein.- Fr.- } \\
\text { Wilhs.-U Bonn }\end{array}$ & 0 & 1 & 1 & 0 & \\
\hline $\begin{array}{l}\text { Evaluating Reported Determinants of } \\
\text { Labor Demand }\end{array}$ & $\begin{array}{l}\text { Ross,-David-R.; } \\
\text { Zimmermann,-Klaus-F. }\end{array}$ & $\begin{array}{l}\text { Labour-Economics; } 1(1), \\
\text { June 1993, pp. 71-84. }\end{array}$ & 1993 & Zimmermann & Klaus F. & \begin{tabular}{|l|} 
Rhein.- Fr.- \\
Wilhs.-U Bonn \\
\end{tabular} & 0 & 1 & 2 & 0 & \\
\hline $\begin{array}{l}\text { Pseudo-R-[superscript 2] Measures } \\
\text { for Some Common Limited } \\
\text { Dependent Variable Models }\end{array}$ & $\begin{array}{l}\text { Veall,-Michael-R.; } \\
\text { Zimmermann,-Klaus-F. }\end{array}$ & $\begin{array}{l}\text { Journal-of-Economic- } \\
\text { Surveys; } 10(3), \\
\text { September 1996, } \\
\text { pp. 241-59. }\end{array}$ & 1996 & Zimmermann & Klaus F. & $\begin{array}{l}\text { Rhein.- Fr.- } \\
\text { Wilhs.-U Bonn }\end{array}$ & 0 & 1 & 2 & 0 & \\
\hline $\begin{array}{l}\text { Tackling the European Migration } \\
\text { Problems }\end{array}$ & Zimmermann,-Klaus-F. & $\begin{array}{l}\text { Journal-of-Economic- } \\
\text { Perspectives; 9(2), } \\
\text { Spring 1995, pp. 45-62. }\end{array}$ & 1995 & Zimmermann & Klaus F. & \begin{tabular}{|l|} 
Rhein.- Fr.- \\
Wilhs.-U Bonn
\end{tabular} & 2.79 & 1 & 1 & 2.79 & \\
\hline $\begin{array}{l}\text { Flexibility in the Face of Demand } \\
\text { Fluctuations: Employment, Capacity } \\
\text { Utilization, and Industry Structure }\end{array}$ & Zimmermann,-Klaus-F. & \begin{tabular}{|l|} 
International-Journal-of- \\
Industrial-Organization; \\
13(2), 1995, pp. 179-93. \\
\end{tabular} & 1995 & Zimmermann & Klaus F. & \begin{tabular}{|l|} 
Rhein.- Fr.- \\
Wilhs.-U Bonn
\end{tabular} & 0.34 & 1 & 1 & 0.34 & \\
\hline $\begin{array}{l}\text { Recent Developments in Count Data } \\
\text { Modelling: Theory and Application }\end{array}$ & $\begin{array}{l}\text { Winkelmann,-Rainer; } \\
\text { Zimmermann,-Klaus-F. }\end{array}$ & $\begin{array}{l}\text { Journal-of-Economic- } \\
\text { Surveys; } 9(1), \text { March } \\
\text { 1995, pp. 1-24. }\end{array}$ & 1995 & Zimmermann & Klaus F. & $\begin{array}{l}\text { Rhein.- Fr.- } \\
\text { Wilhs.-U Bonn }\end{array}$ & 0 & 1 & 2 & 0 & \\
\hline $\begin{array}{l}\text { Goodness-of-Fit Measures in Binary } \\
\text { Choice Models: Comments }\end{array}$ & $\begin{array}{l}\text { Veall,-Michael-R.; } \\
\text { Zimmermann,-Klaus-F. }\end{array}$ & $\begin{array}{l}\text { Econometric-Reviews; } \\
\text { 14(1), February 1995, } \\
\text { pp. 117-20. }\end{array}$ & 1995 & Zimmermann & Klaus F. & $\begin{array}{l}\text { Rhein.- Fr.- } \\
\text { Wilhs.-U Bonn }\end{array}$ & 0 & 0.5 & 2 & 0 & \\
\hline $\begin{array}{l}\text { Pennies from Heaven: Entry Fees to } \\
\text { a Socialist "Paradise." }\end{array}$ & \begin{tabular}{|l|} 
Zimmermann,-Klaus-- \\
F.; Cusack,-Thomas-R.
\end{tabular} & $\begin{array}{l}\text { Public-Choice; 81(3-4), } \\
\text { December 1994, } \\
\text { pp. 277-95. } \\
\end{array}$ & 1994 & Zimmermann & Klaus F. & $\begin{array}{l}\text { Rhein.- Fr.- } \\
\text { Wilhs.-U Bonn }\end{array}$ & 0.43 & 1 & 2 & 0.286666667 & \\
\hline $\begin{array}{l}\text { Goodness of Fit Measures in the } \\
\text { Tobit Model }\end{array}$ & \begin{tabular}{|l|} 
Veall,-Michael-R.; \\
Zimmermann,-Klaus-F.
\end{tabular} & $\begin{array}{l}\text { Oxford-Bulletin-of- } \\
\text { Economics-and- } \\
\text { Statistics; 56(4), } \\
\text { November 1994, } \\
\text { pp. 485-99. } \\
\end{array}$ & 1994 & Zimmermann & Klaus F. & $\begin{array}{l}\text { Rhein.- Fr.- } \\
\text { Wilhs.-U Bonn }\end{array}$ & 0.76 & 1 & 2 & 0.506666667 & \\
\hline $\begin{array}{l}\text { Mass Migration, Unions, and } \\
\text { Government Intervention }\end{array}$ & $\begin{array}{l}\text { Schmidt,-Christoph-M.; } \\
\text { Stilz,-Anette; } \\
\text { Zimmermann,-Klaus-F. }\end{array}$ & \begin{tabular}{|l|} 
Journal-of-Public- \\
Economics; 55(2), \\
October 1994, pp. 185- \\
201. \\
\end{tabular} & 1994 & Zimmermann & Klaus F. & $\begin{array}{l}\text { Rhein.- Fr.- } \\
\text { Wilhs.-U Bonn }\end{array}$ & 0.82 & 1 & 3 & 0.41 & \\
\hline $\begin{array}{l}\text { Native Wage Impacts of Foreign } \\
\text { Labor: A Random Effects Panel } \\
\text { Analysis }\end{array}$ & $\begin{array}{l}\text { De-New,-John-P.; } \\
\text { Zimmermann,-Klaus-F. }\end{array}$ & $\begin{array}{l}\text { Journal-of-Population- } \\
\text { Economics; 7(2), 1994, } \\
\text { pp. 177-92. } \\
\end{array}$ & 1994 & Zimmermann & Klaus F. & $\begin{array}{l}\text { Rhein.- Fr.- } \\
\text { Wilhs.-U Bonn }\end{array}$ & 0.44 & 1 & 2 & 0.293333333 & \\
\hline $\begin{array}{l}\text { A Panel Analysis of Job Changes } \\
\text { and Unemployment }\end{array}$ & $\begin{array}{l}\text { Muhleisen,-Martin; } \\
\text { Zimmermann,-Klaus-F. }\end{array}$ & \begin{tabular}{|l|} 
European-Economic- \\
Review; 38(3-4), April \\
1994, pp. 793-801. \\
\end{tabular} & 1994 & Zimmermann & Klaus F. & $\begin{array}{l}\text { Rhein.- Fr.- } \\
\text { Wilhs.-U Bonn }\end{array}$ & 0.84 & 0.5 & 2 & 0.28 & \\
\hline Towards a European Migration Policy & $\begin{array}{l}\text { Straubhaar,-Thomas; } \\
\text { Zimmermann,-Klaus-F. }\end{array}$ & \begin{tabular}{|l|} 
Population-Research- \\
and-Policy-Review; \\
12(3), 1993, pp. 225-41.
\end{tabular} & 1993 & Zimmermann & Klaus F. & \begin{tabular}{|l} 
Rhein.- Fr.- \\
Wilhs.-U Bonn
\end{tabular} & 0 & 1 & 2 & 0 & \\
\hline $\begin{array}{l}\text { Okonomische Konsequenzen der } \\
\text { Migration fur den heimischen } \\
\text { Arbeitsmarkt. }\end{array}$ & Zimmermann,-Klaus-F. & \begin{tabular}{|l|} 
Schweizerische- \\
Zeitschrift-fur- \\
Volkswirtschaft- \\
undStatistik; 129(3), \\
1993, pp. 283-301. \\
\end{tabular} & 1993 & Zimmermann & Klaus F. & \begin{tabular}{|l|} 
Rhein.- Fr.- \\
Wilhs.-U Bonn
\end{tabular} & 0 & 1 & 1 & 0 & \\
\hline
\end{tabular}


Rheinische Friedrich-Wilhelms-Universität Bonn

\begin{tabular}{|c|c|c|c|c|c|c|c|c|c|c|c|}
\hline & Author(s) & Journal & Year & & & & \begin{tabular}{|l|} 
Impact \\
Factor
\end{tabular} & \begin{tabular}{|c|} 
Seiten- \\
korr.
\end{tabular} & Authors & Impact & personal Impact \\
\hline $\begin{array}{l}\text { Relative Export Prices and Firm Size } \\
\text { in Imperfect Markets }\end{array}$ & \begin{tabular}{|l|} 
Pupillo,-Lorenzo; \\
Zimmermann,-Klaus-F.
\end{tabular} & $\begin{array}{l}\text { Open-Economies- } \\
\text { Review; } 2(3), 1991, \\
\text { pp. 295-304. }\end{array}$ & 1991 & Zimmermann & Klaus F. & $\begin{array}{l}\text { Rhein.- Fr.- } \\
\text { Wilhs.-U Bonn }\end{array}$ & 0 & 0.5 & 2 & 0 & \\
\hline $\begin{array}{l}\text { Illusive Persistence in German } \\
\text { Unemployment }\end{array}$ & $\begin{array}{l}\text { Tschernig,-Rolf; } \\
\text { Zimmermann,-Klaus-F. }\end{array}$ & $\begin{array}{l}\text { Recherches- } \\
\text { Economiques-de- } \\
\text { Louvain; 58(3-4), 1992, } \\
\text { pp. 441-53. }\end{array}$ & 1992 & Zimmermann & Klaus F. & $\begin{array}{l}\text { Rhein.- Fr.- } \\
\text { Wilhs.-U Bonn }\end{array}$ & 0 & 1 & 2 & 0 & \\
\hline $\begin{array}{l}\text { The Demographics of Labour } \\
\text { Turnover: A Comparison of Ordinal } \\
\text { Probit and Censored Count Data } \\
\text { Models }\end{array}$ & $\begin{array}{l}\text { Merkle,-Lucie; } \\
\text { Zimmermann,-Klaus-F. }\end{array}$ & $\begin{array}{l}\text { Recherches- } \\
\text { Economiques-de- } \\
\text { Louvain; 58(3-4), 1992, } \\
\text { pp. 283-306. } \\
\end{array}$ & 1992 & Zimmermann & Klaus F. & \begin{tabular}{|l|} 
Rhein.- Fr.- \\
Wilhs.-U Bonn
\end{tabular} & 0 & 1 & 2 & 0 & \\
\hline Gary S. Becker & Zimmermann,-Klaus-F. & $\begin{array}{l}\text { Journal-of-Population- } \\
\text { Economics; 5(4), 1992, } \\
\text { pp. 251-55. }\end{array}$ & 1992 & Zimmermann & Klaus F. & \begin{tabular}{|l|} 
Rhein.- Fr.- \\
Wilhs.-U Bonn
\end{tabular} & 0.44 & 0.5 & 1 & 0.22 & \\
\hline $\begin{array}{l}\text { Performance Measures from } \\
\text { Prediction-Realization Tables }\end{array}$ & \begin{tabular}{|l|} 
Veall,-Michael-R.; \\
Zimmermann,-Klaus-F.
\end{tabular} & $\begin{array}{l}\text { Economics-Letters; } \\
\text { 39(2), June 1992, } \\
\text { pp. 129-34. }\end{array}$ & 1992 & Zimmermann & Klaus F. & \begin{tabular}{|l|} 
Rhein.- Fr.- \\
Wilhs.-U Bonn
\end{tabular} & 0.26 & 0.5 & 2 & 0.086666667 & \\
\hline Determinanten der Patentaktivitat. & \begin{tabular}{|l|} 
Zimmermann,-Klaus- \\
F.; Schwalbach,-- \\
Joachim \\
\end{tabular} & $\begin{array}{l}\text { Ifo-Studien; 37(3-4), } \\
\text { 1991, pp. 201-27. }\end{array}$ & 1991 & Zimmermann & Klaus F. & $\begin{array}{l}\text { Rhein.- Fr.- } \\
\text { Wilhs.-U Bonn }\end{array}$ & 0 & 1 & 2 & 0 & \\
\hline $\begin{array}{l}\text { Mikrookonomie, Mikrookonometrie } \\
\text { und Wirtschaftspolitik. }\end{array}$ & $\begin{array}{l}\text { Ronning,-Gerd; } \\
\text { Zimmermann,-Klaus-F. }\end{array}$ & $\begin{array}{l}\text { Ifo-Studien; 37(3-4), } \\
\text { 1991, pp. 189-200. }\end{array}$ & 1991 & Zimmermann & Klaus F. & \begin{tabular}{|l|} 
Rhein.- Fr.- \\
Wilhs.-U Bonn
\end{tabular} & 0 & 1 & 2 & 0 & \\
\hline $\begin{array}{l}\text { Savings, Remittances, and Return } \\
\text { Migration }\end{array}$ & $\begin{array}{l}\text { Merkle,-Lucie; } \\
\text { Zimmermann,-Klaus-F. }\end{array}$ & $\begin{array}{l}\text { Economics-Letters; } \\
\text { 38(1), January 1992, } \\
\text { pp. 77-81. }\end{array}$ & 1992 & Zimmermann & Klaus F. & $\begin{array}{l}\text { Rhein.- Fr.- } \\
\text { Wilhs.-U Bonn }\end{array}$ & 0.26 & 0.5 & 2 & 0.086666667 & \\
\hline $\begin{array}{l}\text { Work Characteristics, Firm Size and } \\
\text { Wages }\end{array}$ & $\begin{array}{l}\text { Schmidt,-Christoph-M.; } \\
\text { Zimmermann,-Klaus-F. }\end{array}$ & $\begin{array}{l}\text { Review -of-Economics- } \\
\text { and-Statistics; 73(4), } \\
\text { November 1991, } \\
\text { pp. 705-10. }\end{array}$ & 1991 & Zimmermann & Klaus F. & \begin{tabular}{|l|} 
Rhein.- Fr.- \\
Wilhs.-U Bonn
\end{tabular} & 1.04 & 0.5 & 2 & 0.346666667 & \\
\hline $\begin{array}{l}\text { A New Approach for Modeling } \\
\text { Economic Count Data }\end{array}$ & $\begin{array}{l}\text { Winkelmann,-Rainer; } \\
\text { Zimmermann,-Klaus-F. }\end{array}$ & $\begin{array}{l}\text { Economics-Letters; } \\
37(2), \text { October 1991, } \\
\text { pp. 139-43. } \\
\end{array}$ & 1991 & Zimmermann & Klaus F. & $\begin{array}{l}\text { Rhein.- Fr.- } \\
\text { Wilhs.-U Bonn }\end{array}$ & 0.26 & 0.5 & 2 & 0.086666667 & \\
\hline $\begin{array}{l}\text { Ageing and the Labor Market: Age } \\
\text { Structure, Cohort Size and } \\
\text { Unemployment }\end{array}$ & Zimmermann,-Klaus-F. & $\begin{array}{l}\text { Journal-of-Population- } \\
\text { Economics; 4(3), August } \\
\text { 1991, pp. } 177-200 .\end{array}$ & 1991 & Zimmermann & Klaus F. & $\begin{array}{l}\text { Rhein.- Fr.- } \\
\text { Wilhs.-U Bonn }\end{array}$ & 0.44 & 1 & 1 & 0.44 & \\
\hline $\begin{array}{l}\text { Ein Poisson-Modell zur Schatzung } \\
\text { von Produktionsfunktionen neuen } \\
\text { Wissens. }\end{array}$ & $\begin{array}{l}\text { Schwalbach,-Joachim; } \\
\text { Zimmermann,-Klaus-F. }\end{array}$ & $\begin{array}{l}\text { Zeitschrift-fur- } \\
\text { Betriebswirtschaft; } 61(4), \\
\text { April 1991, pp. 441-49. }\end{array}$ & 1991 & Zimmermann & Klaus F. & $\begin{array}{l}\text { Rhein.- Fr.- } \\
\text { Wilhs.-U Bonn }\end{array}$ & 0 & 0.5 & 2 & 0 & \\
\hline $\begin{array}{l}\text { The Employment Consequences of } \\
\text { Technological Advance, Demand and } \\
\text { Labor Costs in } 16 \text { German Industries }\end{array}$ & Zimmermann,-Klaus-F. & \begin{tabular}{|l|} 
Empirical-Economics; \\
16(2), 1991, pp. 253-66.
\end{tabular} & 1991 & Zimmermann & Klaus F. & \begin{tabular}{|l} 
Rhein.- Fr.- \\
Wilhs.-U Bonn
\end{tabular} & 0 & 1 & 1 & 0 & \\
\hline $\begin{array}{l}\text { Der Ifo-Konjunkturtest in der arbeits- } \\
\text { und industrieokonomischen } \\
\text { Forschung. }\end{array}$ & Zimmermann,-Klaus-F. & $\begin{array}{l}\text { Ifo-Studien; 36(1), 1990, } \\
\text { pp. 1-16. }\end{array}$ & 1990 & Zimmermann & Klaus F. & \begin{tabular}{|l} 
Rhein.- Fr.- \\
Wilhs.-U Bonn
\end{tabular} & 0 & 1 & 1 & 0 & $\begin{array}{l}\text { K. F. Zimmermann: } \\
6.533333333\end{array}$ \\
\hline
\end{tabular}

Average: 0.910857143 
TU-Wien

\begin{tabular}{|c|c|c|c|c|c|c|c|c|c|c|c|}
\hline & Author(s) & Journal & Year & & & & $\begin{array}{l}\text { Impact } \\
\text { Factor }\end{array}$ & $\begin{array}{c}\text { Seiten- } \\
\text { korr }\end{array}$ & Autoren & Impact & personal Impact \\
\hline The Hahn-Solow Macro Model & Frisch,-Helmut & \begin{tabular}{|l|} 
Journal-of-Evolutionary- \\
Economics; 9(2), April \\
1999, pp. 265-70. \\
\end{tabular} & 1999 & Frisch & Helmut & TU-Wien & 0 & 0.5 & 1 & 0 & \\
\hline The Algebra of Government Debt & Frisch,-Helmut & $\begin{array}{l}\text { FinanzArchiv; 54(4), 1997, } \\
\text { pp. 586-99. }\end{array}$ & 1997 & Frisch & Helmut & TU-Wien & 0 & 1 & 1 & 0 & \\
\hline $\begin{array}{l}\text { Government Debt and Sustainable } \\
\text { Fiscal Policy }\end{array}$ & Frisch,-Helmut & $\begin{array}{l}\text { Economic-Notes; 24(3), } \\
\text { 1995, pp. 561-80. }\end{array}$ & 1995 & Frisch & Helmut & TU-Wien & 0 & 1 & 1 & 0 & \\
\hline External Supply Shocks and Stagflation & Frisch,-Helmut & $\begin{array}{l}\text { Economic-Notes; 21(2), } \\
\text { 1992, pp. 225-37. }\end{array}$ & 1992 & Frisch & Helmut & TU-Wien & 0 & 1 & 1 & 0 & \\
\hline $\begin{array}{l}\text { Imported Materials Prices, Wage Policy } \\
\text { and Macroeconomic Stabilization: A } \\
\text { Note }\end{array}$ & $\begin{array}{l}\text { Frisch,-Helmut; } \\
\text { Hof,-Franz-Xaver }\end{array}$ & $\begin{array}{l}\text { Scandinavian-Journal-of- } \\
\text { Economics; 93(4), 1991, } \\
\text { pp. 571-76. }\end{array}$ & 1991 & Frisch & Helmut & TU-Wien & 0.48 & 0.5 & 2 & 0.16 & \\
\hline $\begin{array}{l}\text { Imported Material Price Shocks under } \\
\text { Alternative Wage Regimes }\end{array}$ & $\begin{array}{l}\text { Frisch,-Helmut; } \\
\text { Hof,-Franz-Xaver }\end{array}$ & $\begin{array}{l}\text { Economic-Notes; 20(2), } \\
\text { 1991, pp. 229-53. }\end{array}$ & 1991 & Frisch & Helmut & TU-Wien & 0 & 1 & 2 & 0 & H. Frisch: 0.16 \\
\hline $\begin{array}{l}\text { Evolutionism in Natural Sciences and } \\
\text { Social Sciences: On Basic Concepts of } \\
\text { Evolutionary Economics }\end{array}$ & Hanappi,-Gerhard & $\begin{array}{l}\text { Methodus; 4(1), June } \\
\text { 1992, pp. 110-17. }\end{array}$ & 1992 & Hanappi & Gerhard & TU-Wien & 0 & 0.5 & 1 & 0 & G. Hanappi: 0 \\
\hline $\begin{array}{l}\text { Imported Materials Prices, Wage Policy } \\
\text { and Macroeconomic Stabilization: A } \\
\text { Note }\end{array}$ & $\begin{array}{l}\text { Frisch,-Helmut; } \\
\text { Hof,-Franz-Xaver }\end{array}$ & \begin{tabular}{|l} 
Scandinavian-Journal-of- \\
Economics; 93(4), 1991, \\
pp. 571-76. \\
\end{tabular} & 1991 & Hof & Franz X. & TU-Wien & 0.48 & 0.5 & 2 & 0.16 & \\
\hline $\begin{array}{l}\text { Imported Material Price Shocks under } \\
\text { Alternative Wage Regimes }\end{array}$ & $\begin{array}{l}\text { Frisch,-Helmut; } \\
\text { Hof,-Franz-Xaver }\end{array}$ & $\begin{array}{l}\text { Economic-Notes; 20(2), } \\
\text { 1991, pp. 229-53. }\end{array}$ & 1991 & Hof & Franz X. & TU-Wien & 0 & 1 & 2 & 0 & \\
\hline $\begin{array}{l}\text { Wage Bargaining and Shock Sensitivity } \\
\text { of a Small Open Economy }\end{array}$ & $\begin{array}{l}\text { Fehr,-Ernst; Hof,-- } \\
\text { Franz-X. }\end{array}$ & $\begin{array}{l}\text { Journal-of-Economics- } \\
\text { (ZfN); 59(3), 1994, } \\
\text { pp. 259-86. }\end{array}$ & 1994 & Hof & Franz X. & TU-Wien & 0.18 & 1 & 2 & 0.12 & F. X. Hof: 0.28 \\
\hline Do Addicts Behave Rationally? & \begin{tabular}{|l|} 
Fehr,-Ernst; Zych,- \\
Peter-K.
\end{tabular} & $\begin{array}{l}\text { Scandinavian-Journal-of- } \\
\text { Economics; 100(3), } \\
\text { September 1998, pp. 643- } \\
62 . \\
\end{array}$ & 1998 & Zych & Peter K. & TU-Wien & 0.48 & 1 & 2 & 0.32 & \\
\hline $\begin{array}{l}\text { Die Macht der Versuchung: Irrationaler } \\
\text { Uberkonsum in einem } \\
\text { Suchtexperiement. }\end{array}$ & \begin{tabular}{|l|} 
Fehr,-Ernst; Zych,- \\
Peter-K.
\end{tabular} & \begin{tabular}{|l|} 
Zeitschrift-fur-Wirtschafts-- \\
und-Sozialwissenschaften; \\
115(4), 1995, pp. 569-604.
\end{tabular} & 1995 & Zych & Peter K. & TU-Wien & 0 & 1 & 2 & 0 & P. K. Zych: 0.32 \\
\hline
\end{tabular}


Universität Graz

\begin{tabular}{|c|c|c|c|c|c|c|c|c|c|c|c|}
\hline & Author(s) & Journal & Year & & & & $\begin{array}{l}\text { Impact } \\
\text { Factor }\end{array}$ & $\begin{array}{l}\text { Seiten- } \\
\text { korr. }\end{array}$ & Autoren & Impact & personal Impact \\
\hline $\begin{array}{l}\text { Schumpeter's Perception of Austrian } \\
\text { Economics: A Comparison between } \\
\text { Epochen der Dogmen-und } \\
\text { Methodengeschichte ... }\end{array}$ & Beinsen,-Lutz & $\begin{array}{l}\text { History-of-Economic-Ideas; } \\
6(1), 1998, \text { pages 55-77. }\end{array}$ & 1998 & Beinsen & Lutz & Uni-Graz & 0 & 1 & 1 & 0 & L. Beinsen: 0 \\
\hline Why the Acrimony? Reply to Davidson & $\begin{array}{l}\text { Boehm,-Stephan; } \\
\text { Farmer,-Karl }\end{array}$ & $\begin{array}{l}\text { Critical-Review; 7(2-3), } \\
\text { Spring-Summer 1993, } \\
\text { pages 407-21. }\end{array}$ & 1993 & Böhm & Stephan & Uni-Graz & 0 & 1 & 2 & 0 & S. Böhm: 0 \\
\hline $\begin{array}{l}\text { Heterogeneous Capital in a Two-Sector } \\
\text { Overlapping-Generations Model: Reply } \\
\text { to Professor Morishima's Comment }\end{array}$ & Farmer,-Karl & $\begin{array}{l}\text { Metroeconomica; } 48(1), \\
\text { February 1997, pages 58- } \\
59 .\end{array}$ & 1997 & Farmer & Karl & Uni-Graz & 0 & 0.5 & 1 & 0 & \\
\hline $\begin{array}{l}\text { Heterogeneous Capital in a Two-Sector } \\
\text { Overlapping-Generations Model }\end{array}$ & Farmer,-Karl & $\begin{array}{l}\text { Metroeconomica; 48(1), } \\
\text { February 1997, pages } 36- \\
54 .\end{array}$ & 1997 & Farmer & Karl & Uni-Graz & 0 & 1 & 1 & 0 & \\
\hline Why the Acrimony? Reply to Davidson & $\begin{array}{l}\text { Boehm,-Stephan; } \\
\text { Farmer,-Karl }\end{array}$ & $\begin{array}{l}\text { Critical-Review; 7(2-3), } \\
\text { Spring-Summer 1993, } \\
\text { pages 407-21. }\end{array}$ & 1993 & Farmer & Karl & Uni-Graz & 0 & 1 & 2 & 0 & K. Farmer: 0 \\
\hline Metroeconomica at 50 & $\begin{array}{l}\text { Kurz,-Heinz-D.; } \\
\text { Gehrke,-Christian }\end{array}$ & $\begin{array}{l}\text { Metroeconomica; } 50(3), \\
\text { October 1999, pages 255- } \\
59 .\end{array}$ & 1999 & Gehrke & Christian & Uni-Graz & 0 & 0.5 & 2 & 0 & \\
\hline $\begin{array}{l}\text { Introduction to Adolf Lowe's 'How Is } \\
\text { Business Cycle Theory Possible at All?' }\end{array}$ & Gehrke,-Christian & \begin{tabular}{|l|} 
Structural-Change-and- \\
Economic-Dynamics; $8(2)$, \\
June 1997, pages 233-44. \\
\end{tabular} & 1997 & Gehrke & Christian & Uni-Graz & 0 & 1 & 1 & 0 & \\
\hline $\begin{array}{l}\text { On the Choice of Technique: A } \\
\text { Comment }\end{array}$ & $\begin{array}{l}\text { Kurz,-Heinz-D.; } \\
\text { Gehrke,-Christian }\end{array}$ & \begin{tabular}{l|} 
Jahrbucher-fur- \\
Nationalokonomie-und- \\
Statistik; 213(1), Jan. 1994, \\
pp. 100-106.
\end{tabular} & 1994 & Gehrke & Christian & Uni-Graz & 0.21 & 0.5 & 2 & 0.07 & \\
\hline Karl Marx on Physiocracy & $\begin{array}{l}\text { Gehrke,-Christian; } \\
\text { Kurz,-Heinz-D. }\end{array}$ & $\begin{array}{l}\text { European-Journal-of-the- } \\
\text { History-of-Economic- } \\
\text { Thought; 2(1), 1995, pp. } \\
\text { 53-90. }\end{array}$ & 1995 & Gehrke & Christian & Uni-Graz & 0 & 1 & 2 & 0 & \\
\hline $\begin{array}{l}\text { Environmental Taxes, Relative Prices } \\
\text { and Choice of Technique in a Linear } \\
\text { Model of Production }\end{array}$ & $\begin{array}{l}\text { Gehrke,-Christian; } \\
\text { Lager,-Christian }\end{array}$ & $\begin{array}{l}\text { Metroeconomica; 46(2), } \\
\text { June 1995, pages 127-45. }\end{array}$ & 1995 & Gehrke & Christian & Uni-Graz & 0 & 1 & 2 & 0 & C. Gehrke: 0.07 \\
\hline Metroeconomica at 50 & $\begin{array}{l}\text { Kurz,-Heinz-D.; } \\
\text { Gehrke,-Christian }\end{array}$ & $\begin{array}{l}\text { Metroeconomica; } 50(3), \\
\text { October 1999, pages 255- } \\
59 .\end{array}$ & 1999 & Kurz & Heinz & Uni-Graz & 0 & 0.5 & 2 & 0 & \\
\hline $\begin{array}{l}\text { Le fonds Sraffa a Cambridge: Apercu } \\
\text { sur les ecrits nonpublies. (Sraffa's } \\
\text { Contributions to Economics: Some } \\
\text { Notes on His Unpublished Papers.) } \\
\end{array}$ & Kurz,-Heinz-D. & $\begin{array}{l}\text { Revue-d'Economie- } \\
\text { Politique; } 108(6) \text {, Nov-- } \\
\text { Dec. 1998, pages 813-37. }\end{array}$ & 1998 & Kurz & Heinz & Uni-Graz & 0 & 1 & 1 & 0 & \\
\hline $\begin{array}{l}\text { Against the Current: Sraffa's } \\
\text { Unpublished Manuscripts and the } \\
\text { History of Economic Thought }\end{array}$ & Kurz,-Heinz-D. & $\begin{array}{l}\text { European-Journal-of-the- } \\
\text { History-of-Economic- } \\
\text { Thought; 5(3), 1998, pp. } \\
\text { 437-51. }\end{array}$ & 1998 & Kurz & Heinz & Uni-Graz & 0 & 1 & 1 & 0 & \\
\hline $\begin{array}{l}\text { Reverse Capital Deepening and the } \\
\text { Numeraire: A Note }\end{array}$ & $\begin{array}{l}\text { Kurz,-Heinz-D.; } \\
\text { Salvadori,-Neri }\end{array}$ & $\begin{array}{l}\text { Review -of-Political- } \\
\text { Economy; 10(4), October } \\
\text { 1998, pages 415-26. } \\
\end{array}$ & 1998 & Kurz & Heinz & Uni-Graz & 0 & 1 & 2 & 0 & \\
\hline
\end{tabular}


Universität Graz

\begin{tabular}{|c|c|c|c|c|c|c|c|c|c|c|c|}
\hline & Author(s) & Journal & Year & & & & $\begin{array}{l}\text { Impact } \\
\text { Factor }\end{array}$ & $\begin{array}{l}\text { Seiten- } \\
\text { korr. }\end{array}$ & Autoren & Impact & personal Impact \\
\hline $\begin{array}{l}\text { Friedrich Benedikt Wilhelm Hermann on } \\
\text { Capital and Profits }\end{array}$ & Kurz,-Heinz-D. & $\begin{array}{l}\text { European-Journal-of-the- } \\
\text { History-of-Economic- } \\
\text { Thought; 5(1), 1998, pp. } \\
85-119 .\end{array}$ & 1998 & Kurz & Heinz & Uni-Graz & 0 & 1 & 1 & 0 & \\
\hline Morishima on Ricardo: A Rejoinder & $\begin{array}{l}\text { Kurz,-Heinz-D.; } \\
\text { Salvadori,-Neri }\end{array}$ & $\begin{array}{l}\text { Cambridge-Journal-of- } \\
\text { Economics; 22(2), March } \\
\text { 1998, pages 227-39. }\end{array}$ & 1998 & Kurz & Heinz & Uni-Graz & 0.71 & 1 & 2 & 0.47333333 & \\
\hline $\begin{array}{l}\text { Exhaustible Resources in a Dynamic } \\
\text { Input-Output Model with 'Classical' } \\
\text { Resources }\end{array}$ & $\begin{array}{l}\text { Kurz,-Heinz-D.; } \\
\text { Salvadori,-Neri }\end{array}$ & \begin{tabular}{|l|} 
Economic-Systems - \\
Research; 9(3), September \\
1997, pages 235-51.
\end{tabular} & 1997 & Kurz & Heinz & Uni-Graz & 0 & 1 & 2 & 0 & \\
\hline $\begin{array}{l}\text { What Could the 'New' Growth Theory } \\
\text { Teach Smith or Ricardo? }\end{array}$ & Kurz,-Heinz-D. & $\begin{array}{l}\text { Economic-Issues; } 2(2), \\
\text { September 1997, pages 1- } \\
20 .\end{array}$ & 1997 & Kurz & Heinz & Uni-Graz & 0 & 1 & 1 & 0 & \\
\hline $\begin{array}{l}\text { Sulle perturbazioni "naturali" e } \\
\text { "artificiali" dell'equilibrio economico } \\
\text { generale. La teoria monetaria del } \\
\text { sovrainvestimento di Friedrich August } \\
\text { Hayek... }\end{array}$ & Kurz,-Heinz-D. & $\begin{array}{l}\text { Studi-Economici; 50(56), } \\
\text { 1995, pages 5-61. }\end{array}$ & 1995 & Kurz & Heinz & Uni-Graz & 0 & 1 & 1 & 0 & \\
\hline $\begin{array}{l}\text { F. Helmedag und die "okonomische } \\
\text { Logik." }\end{array}$ & Kurz,-Heinz-D. & $\begin{array}{l}\text { Jahrbucher-fur- } \\
\text { Nationalokonomie-und- } \\
\text { Statistik; } 214(6) \text {, Nov. } \\
\text { 1995, pp. } 710-27 . \\
\end{array}$ & 1995 & Kurz & Heinz & Uni-Graz & 0.21 & 1 & 1 & 0.21 & \\
\hline Growth and Distribution & Kurz,-Heinz-D. & \begin{tabular}{|l|} 
Review -of-Political- \\
Economy; 6(4), October \\
1994, pages 393-420.
\end{tabular} & 1994 & Kurz & Heinz & Uni-Graz & 0 & 1 & 1 & 0 & \\
\hline $\begin{array}{l}\text { On the Choice of Technique: A } \\
\text { Comment }\end{array}$ & $\begin{array}{l}\text { Kurz,-Heinz-D.; } \\
\text { Gehrke,-Christian }\end{array}$ & \begin{tabular}{l|} 
Jahrbucher-fur- \\
Nationalokonomie-und- \\
Statistik; 213(1), Jan. 1994, \\
pp. 100-106. \\
\end{tabular} & 1994 & Kurz & Heinz & Uni-Graz & 0.21 & 0.5 & 2 & 0.07 & \\
\hline Karl Marx on Physiocracy & $\begin{array}{l}\text { Gehrke,-Christian; } \\
\text { Kurz,-Heinz-D. }\end{array}$ & $\begin{array}{l}\text { European-Journal-of-the- } \\
\text { History-of-Economic- } \\
\text { Thought; 2(1), 1995, pp. } \\
\text { 53-90. }\end{array}$ & 1995 & Kurz & Heinz & Uni-Graz & 0 & 1 & 2 & 0 & \\
\hline $\begin{array}{l}\text { Choice of Technique in a Model with } \\
\text { Fixed Capital }\end{array}$ & $\begin{array}{l}\text { Kurz,-Heinz-D.; } \\
\text { Salvadori,-Neri }\end{array}$ & $\begin{array}{l}\text { European-Journal-of- } \\
\text { Political-Economy; } 10(3), \\
\text { October 1994, pages 545- } \\
69 .\end{array}$ & 1994 & Kurz & Heinz & Uni-Graz & 0 & 1 & 2 & 0 & \\
\hline $\begin{array}{l}\text { Von Neumann's Growth Model and the } \\
\text { 'Classical' Tradition }\end{array}$ & $\begin{array}{l}\text { Kurz,-Heinz-D.; } \\
\text { Salvadori,-Neri }\end{array}$ & $\begin{array}{l}\text { European-Journal-of-the- } \\
\text { History-of-Economic- } \\
\text { Thought; 1(1), 1993, pp. } \\
\text { 129-60. } \\
\end{array}$ & 1993 & Kurz & Heinz & Uni-Graz & 0 & 1 & 2 & 0 & \\
\hline $\begin{array}{l}\text { The Non-substitution Theorem: Making } \\
\text { Good a Lacuna }\end{array}$ & $\begin{array}{l}\text { Kurz,-Heinz-D.; } \\
\text { Salvadori,-Neri }\end{array}$ & $\begin{array}{l}\text { Journal-of-Economics- } \\
\text { (Zeitschrift-fur- } \\
\text { Nationalokonomie); 59(1), } \\
\text { 1994, p. 97-103. }\end{array}$ & 1994 & Kurz & Heinz & Uni-Graz & 0.18 & 0.5 & 2 & 0.06 & \\
\hline
\end{tabular}


Universität Graz

\begin{tabular}{|c|c|c|c|c|c|c|c|c|c|c|c|}
\hline & Author(s) & Journal & Year & & & & $\begin{array}{l}\text { Impact } \\
\text { Factor }\end{array}$ & $\begin{array}{l}\text { Seiten- } \\
\text { korr. }\end{array}$ & Autoren & Impact & personal Impact \\
\hline $\begin{array}{l}\text { Micro-electronics and Employment: A } \\
\text { Dynamic Input-Output Study of the } \\
\text { West German Economy }\end{array}$ & $\begin{array}{l}\text { Kalmbach,-Peter; } \\
\text { Kurz,-Heinz-D. }\end{array}$ & $\begin{array}{l}\text { Structural-Change-and- } \\
\text { Economic-Dynamics; } 1(2), \\
\text { Dec. 1990, pp. 371-86. }\end{array}$ & 1990 & Kurz & Heinz & Uni-Graz & 0 & 1 & 2 & 0 & \\
\hline $\begin{array}{l}\text { Adam Smith on Foreign Trade: A Note } \\
\text { on the 'Vent-for-Surplus' Argument }\end{array}$ & Kurz,-Heinz-D. & $\begin{array}{l}\text { Economica; } 59(236) \\
\text { November 1992, pages } \\
475-81 .\end{array}$ & 1992 & Kurz & Heinz & Uni-Graz & 0.51 & 0.5 & 1 & 0.255 & \\
\hline Morishima on Ricardo: Review Article & $\begin{array}{l}\text { Kurz,-Heinz-D.; } \\
\text { Salvadori,-Neri }\end{array}$ & $\begin{array}{l}\text { Cambridge-Journal-of- } \\
\text { Economics; 16(2), June } \\
\text { 1992, pages 227-47. }\end{array}$ & 1992 & Kurz & Heinz & Uni-Graz & 0.71 & 1 & 2 & 0.47333333 & \\
\hline $\begin{array}{l}\text { Effective Demand, Employment and } \\
\text { Capital Utilisation in the Short Run }\end{array}$ & Kurz,-Heinz-D. & $\begin{array}{l}\text { Cambridge-Journal-of- } \\
\text { Economics; } 14(2), \text { June } \\
\text { 1990, pages 205-17. } \\
\end{array}$ & 1990 & Kurz & Heinz & Uni-Graz & 0.71 & 1 & 1 & 0.71 & $\begin{array}{l}\text { H. Kurz: } \\
2.251666667\end{array}$ \\
\hline On the Notion of the Rate of Profit & Lager,-Christian & $\begin{array}{l}\text { Review-of-Political- } \\
\text { Economy; 10(4), October } \\
\text { 1998, pages 447-58. }\end{array}$ & 1998 & Lager & Christian & Uni-Graz & 0 & 1 & 1 & 0 & \\
\hline $\begin{array}{l}\text { Prices of 'Goods' and 'Bads': An } \\
\text { Application of the Ricardian Theory of } \\
\text { Differential Rent }\end{array}$ & Lager,-Christian & $\begin{array}{l}\text { Economic-Systems - } \\
\text { Research; 10(3), } \\
\text { September 1998, pages } \\
\text { 203-22. } \\
\end{array}$ & 1998 & Lager & Christian & Uni-Graz & 0 & 1 & 1 & 0 & \\
\hline $\begin{array}{l}\text { Treatment of Fixed Capital in the } \\
\text { Sraffian Framework and in the Theory } \\
\text { of Dynamic Input-Output Models }\end{array}$ & Lager,-Christian & \begin{tabular}{l|} 
Economic-Systems - \\
Research; 9(4), December \\
1997, pages 357-73.
\end{tabular} & 1997 & Lager & Christian & Uni-Graz & 0 & 1 & 1 & 0 & \\
\hline $\begin{array}{l}\text { Auch mit viel Fleiss--Kein Preis oder } \\
\text { Einstimmigkeit ist nicht leicht zu } \\
\text { erzielen. Eine Antwort }\end{array}$ & Lager,-Christian & $\begin{array}{l}\text { Jahrbucher-fur- } \\
\text { Nationalokonomie-und- } \\
\text { Statistik; 214(6), Nov. } \\
\text { 1995, pp. 728-31. } \\
\end{array}$ & 1995 & Lager & Christian & Uni-Graz & 0.21 & 0.5 & 1 & 0.105 & \\
\hline $\begin{array}{l}\text { Uber einen weiteren vergeblichen } \\
\text { Versuch zur Rechtfertigung der } \\
\text { Arbeitswertlehre: Ein Kommmentar }\end{array}$ & Lager,-Christian & \begin{tabular}{l|} 
Jahrbucher-fur- \\
Nationalokonomie-und- \\
Statistik; 214(4), July 1995, \\
pages 463-69.
\end{tabular} & 1995 & Lager & Christian & Uni-Graz & 0.21 & 1 & 1 & 0.21 & \\
\hline $\begin{array}{l}\text { Environmental Taxes, Relative Prices } \\
\text { and Choice of Technique in a Linear } \\
\text { Model of Production }\end{array}$ & $\begin{array}{l}\text { Gehrke,-Christian; } \\
\text { Lager,-Christian }\end{array}$ & $\begin{array}{l}\text { Metroeconomica; 46(2), } \\
\text { June 1995, pages 127-45. }\end{array}$ & 1995 & Lager & Christian & Uni-Graz & 0 & 1 & 2 & 0 & C. Lager: 0.315 \\
\hline $\begin{array}{l}\text { Impact of Carbon Dioxide Emissions } \\
\text { Reduction on the Austrian Economy }\end{array}$ & \begin{tabular}{|l|} 
Kratena,-Kurt; \\
Schleicher,-Stefan
\end{tabular} & $\begin{array}{l}\text { Economic-Systems - } \\
\text { Research; 11(3), } \\
\text { September 1999, pages } \\
245-61 .\end{array}$ & 1999 & Schleicher & Stefan & $\begin{array}{l}\text { Uni-Graz, } \\
\text { Wifo }\end{array}$ & 0 & 1 & 2 & 0 & \\
\hline $\begin{array}{l}\text { Endogenous Technical Progress } \\
\text { Induced by CO2 Reduction Policies: } \\
\text { Simulation Results for Austria }\end{array}$ & $\begin{array}{l}\text { Glueck,-H.; } \\
\text { Schleicher,-S.-P. }\end{array}$ & \begin{tabular}{|l|} 
Environmental-and- \\
Resource-Economics; 5(2), \\
March 1995, pages 151-63.
\end{tabular} & 1995 & Schleicher & Stefan & $\begin{array}{l}\text { Uni-Graz, } \\
\text { Wifo }\end{array}$ & 0 & 1 & 2 & 0 & S. Schleicher: 0 \\
\hline $\begin{array}{l}\text { Labor Market Performance and } \\
\text { Regional Types: A Conceptual } \\
\text { Framework with Empirical Analysis of } \\
\text { Austria }\end{array}$ & $\begin{array}{l}\text { Kubin,-Ingrid; } \\
\text { Steiner,-Michael }\end{array}$ & $\begin{array}{l}\text { International-Regional- } \\
\text { Science-Review; 14(3), } \\
\text { 1992, pages 275-98. }\end{array}$ & 1992 & Steiner & Michael & Uni-Graz & 0 & 1 & 2 & 0 & M. Steiner: 0 \\
\hline $\begin{array}{l}\text { From Ownership to Service Use } \\
\text { Lifestyle: The Potential of Car Sharing }\end{array}$ & $\begin{array}{l}\text { Prettenthaler,-F.- } \\
\text { E.; Steininger,-K.- } \\
\text { W. }\end{array}$ & $\begin{array}{l}\text { Ecological-Economics; } \\
\text { 28(3), March 1999, pages } \\
\text { 443-53. } \\
\end{array}$ & 1999 & Steininger & Karl & Uni-Graz & 0.64 & 1 & 2 & 0.42666666 & \\
\hline
\end{tabular}


Universität Graz

\begin{tabular}{|c|c|c|c|c|c|c|c|c|c|c|c|}
\hline & Author(s) & Journal & Year & & & & $\begin{array}{l}\text { Impact } \\
\text { Factor }\end{array}$ & $\begin{array}{l}\text { Seiten- } \\
\text { korr. }\end{array}$ & Autoren & Impact & personal Impact \\
\hline $\begin{array}{l}\text { Spatial Discounting and the } \\
\text { Environment: An Empirical } \\
\text { Investigation into Human Preferences }\end{array}$ & Steininger,-Karl & $\begin{array}{l}\text { Indian-Journal-of-Applied- } \\
\text { Economics; 7(2), April- } \\
\text { June 1998, pages 253-68. }\end{array}$ & 1998 & Steininger & Karl & Uni-Graz & 0 & 1 & 1 & 0 & \\
\hline $\begin{array}{l}\text { Biomass Energy Use to Reduce } \\
\text { Climate Change: A General Equilibrium } \\
\text { Analysis for Austria }\end{array}$ & $\begin{array}{l}\text { Breuss,-Fritz; } \\
\text { Steininger,-Karl }\end{array}$ & $\begin{array}{l}\text { Journal-of-Policy-Modeling; } \\
\text { 20(4), August 1998, pages } \\
\text { 513-35. }\end{array}$ & 1998 & Steininger & Karl & Uni-Graz & 0.12 & 1 & 2 & 0.08 & $\begin{array}{l}\text { K. Steininger: } \\
0.506666667\end{array}$ \\
\hline $\begin{array}{l}\text { Postsocialist Privatization and Agency- } \\
\text { Related Property: From Coase to } \\
\text { Locke }\end{array}$ & Sturn,-Richard & $\begin{array}{l}\text { European-Journal-of-the- } \\
\text { History-of-Economic- } \\
\text { Thought; } 1(1), 1993, p p . \\
63-86 .\end{array}$ & 1993 & Sturn & Richard & Uni-Graz & 0 & 1 & 1 & 0 & \\
\hline $\begin{array}{l}\text { Diskriminierung als Kontraktproblem. } \\
\text { Zur Mikrookonomik der } \\
\text { Chancengleichheit auf dem Markt fur } \\
\text { Fuhrungskrafte. }\end{array}$ & $\begin{array}{l}\text { Sturn,-Dorothea; } \\
\text { Sturn,-Richard }\end{array}$ & $\begin{array}{l}\text { Kyklos; 45(4), 1992, pages } \\
\text { 483-500. }\end{array}$ & 1992 & Sturn & Richard & Uni-Graz & 0.37 & 1 & 2 & 0.24666666 & $\begin{array}{l}\text { R. Sturn: } \\
0.246666667\end{array}$ \\
\hline $\begin{array}{l}\text { A Calibration Procedure of Dynamic } \\
\text { CGE Models for Non-steady State } \\
\text { Situations Using GEMPACK }\end{array}$ & Wendner,-Ronald & $\begin{array}{l}\text { Computational-Economics; } \\
\text { 13(3), June 1999, pages } \\
\text { 265-87. } \\
\end{array}$ & 1999 & Wendner & Ronald & Uni-Graz & 0 & 1 & 1 & 0 & R. Wender: 0 \\
\hline Behind the Veil of Preference & Baigent,-Nick & $\begin{array}{l}\text { Japanese-Economic- } \\
\text { Review; 46(1), March } \\
\text { 1995, pages 88-101. }\end{array}$ & 1995 & Baigent & Nick & Uni-Graz & 0 & 1 & 1 & 0 & \\
\hline $\begin{array}{l}\text { Never Choose the Uniquely Largest: } \mathrm{A} \\
\text { Characterization }\end{array}$ & $\begin{array}{l}\text { Baigent,-Nick; } \\
\text { Gaertner,-Wulf }\end{array}$ & $\begin{array}{l}\text { Economic-Theory; } 8(2), \\
\text { August } 1996, \text { pages } 239 \text { - } \\
49 \text {. }\end{array}$ & 1996 & Baigent & Nick & Uni-Graz & 0 & 0.5 & 2 & 0 & \\
\hline Manipulability of Choice Aggregations & Baigent,-Nick & $\begin{array}{l}\text { Economics-Letters; } 38(2) \\
\text { February 1992, pages 195- } \\
98 .\end{array}$ & 1992 & Baigent & Nick & $\begin{array}{l}\text { Queen } \\
\text { Mary \& } \\
\text { Westfield } \\
\text { Coll. }\end{array}$ & 0.26 & 0.5 & 1 & 0.13 & \\
\hline $\begin{array}{l}\text { Independent Necessary and Sufficient } \\
\text { Conditions for Approval Voting }\end{array}$ & $\begin{array}{l}\text { Baigent,-Nick; Xu,- } \\
\text { Yongsheng }\end{array}$ & $\begin{array}{l}\text { Mathematical-Social- } \\
\text { Sciences; 21(1), February } \\
\text { 1991, pages 21-29. }\end{array}$ & 1991 & Baigent & Nick & Tulane U & 0.34 & 0.5 & 2 & 0.11333333 & \\
\hline Transitivity and Consistency & Baigent,-Nick & $\begin{array}{l}\text { Economics-Letters; 33(4), } \\
\text { August 1990, pages 315- } \\
17 .\end{array}$ & 1990 & Baigent & Nick & Tulane U & 0.26 & 0.5 & 1 & 0.13 & \\
\hline $\begin{array}{l}\text { Topological Social Choice: Reply to Le } \\
\text { Breton and Uriarte }\end{array}$ & $\begin{array}{l}\text { Baigent,-N.; } \\
\text { Huang,-P. }\end{array}$ & $\begin{array}{l}\text { Social-Choice-and-Welfare; } \\
7(2), 1990 \text {, pages } 141-46 .\end{array}$ & 1990 & Baigent & Nick & Tulane U & 0.55 & 0.5 & 2 & 0.18333333 & \begin{tabular}{|l|} 
N. Baigent: \\
0.556666667
\end{tabular} \\
\hline
\end{tabular}

Average: $\quad 0.21925925$ 
Universität Innsbruck

Authors(s) Journal Year

Impact Seiten Autoren Impact personal Impact

Factor korr.

\begin{tabular}{|c|c|c|c|c|c|c|c|c|c|c|c|}
\hline $\begin{array}{r}\text { Do People Care } \\
\text { about democracy }\end{array}$ & $\begin{array}{r}\text { Gueth,- } \\
\text { Werner; } \\
\text { Weck- } \\
\text { Hannemann,- } \\
\text { Hannelore } \\
\end{array}$ & $\begin{array}{r}\text { Public Choice; } \\
91(1), \text { April 1997, } \\
\text { pages 27-47 }\end{array}$ & 1997 & $\begin{array}{r}\text { Weck- } \\
\text { Hannemann }\end{array}$ & Hannelore & $\begin{array}{r}\text { Universität } \\
\text { Innsbruck }\end{array}$ & 0,43 & 1 & 2 & $\begin{array}{r}0,28666666 \\
7\end{array}$ & $\begin{array}{r}\text { H. Weck } \\
\text { Hannemann: }\end{array}$ \\
\hline $\begin{array}{r}\text { Tax, Rates, Tax } \\
\text { Administration and } \\
\text { Income Tax Evasion } \\
\text { in Switzerland }\end{array}$ & $\begin{array}{r}\text { Pommerehne, } \\
\text { Werner-W; } \\
\text { Weck- } \\
\text { Hannemann,- } \\
\text { Hannelore }\end{array}$ & $\begin{array}{r}\text { Public Choice; } \\
88(1-2), \text { July } 1996, \\
\text { pages } 161-70 .\end{array}$ & 1996 & $\begin{array}{r}\text { Weck- } \\
\text { Hannemann }\end{array}$ & Hannelore & $\begin{array}{r}\text { Universität } \\
\text { Innsbruck }\end{array}$ & 0,43 & 1 & 2 & $\begin{array}{r}0,28666666 \\
7\end{array}$ & 0,86 \\
\hline Steuerhinterziehung & $\begin{array}{r}\text { Pommerehne, } \\
\text {-Wermer-W; } \\
\text { Weck- } \\
\text { Hannemann,- } \\
\text { Hannelore } \\
\end{array}$ & $\begin{array}{r}\text { Zeitschrift fuer } \\
\text { Wirtschafts- und } \\
\text { Sozialwissenschaft } \\
\text { en; 112(3), 1992, } \\
\text { pages 433-66. }\end{array}$ & 1992 & $\begin{array}{r}\text { Weck- } \\
\text { Hannemann }\end{array}$ & Hannelore & $\begin{array}{r}\text { Universität } \\
\text { Innsbruck }\end{array}$ & 0 & 1 & 2 & 0 & \\
\hline $\begin{array}{r}\text { Protectionism in } \\
\text { direct democracy }\end{array}$ & $\begin{array}{r}\text { Weck- } \\
\text { Hannemann,- } \\
\text { Hannelore }\end{array}$ & $\begin{array}{r}\text { Journal of } \\
\text { Institutional and } \\
\text { theoretical } \\
\text { economics; } 146(3), \\
\text { Sept. 1990, pages } \\
389-418 .\end{array}$ & 1990 & $\begin{array}{r}\text { Weck- } \\
\text { Hannemann }\end{array}$ & Hannelore & $\begin{array}{r}\text { Universität } \\
\text { Innsbruck }\end{array}$ & 0,29 & 1 & 1 & 0,29 & \\
\hline $\begin{array}{l}\text { The Iron Law of } \\
\text { Important Articles }\end{array}$ & $\begin{array}{r}\text { Holub,-Hans- } \\
\text { Werner; } \\
\text { Tappeiner,- } \\
\text { Gottfried.; } \\
\text { Eberharter,- } \\
\text { Veronika }\end{array}$ & $\begin{array}{r}\text { Southern- } \\
\text { Economic-Journal; } \\
58(2) \text {, October } \\
1991 \text {, pages } 317- \\
28 .\end{array}$ & 1991 & Eberharter & Veronika & $\begin{array}{r}\text { Universität } \\
\text { Innsbruck }\end{array}$ & 0,39 & 1 & 3 & 0,195 & $\begin{array}{r}\text { V. Eberharter: } \\
0.195\end{array}$ \\
\hline $\begin{array}{r}\text { Effetti dell'entrata } \\
\text { dell'Austria nella UE } \\
\text { sull'interscambio tra } \\
\text { Tirolo e Trentino- } \\
\text { Alto Adige. }\end{array}$ & $\begin{array}{r}\text { Stefani,- } \\
\text { Giorgio; } \\
\text { Socher,-Karl }\end{array}$ & $\begin{array}{r}\text { Rivista- } \\
\text { Internazionale-di- } \\
\text { Scienze- } \\
\text { Economiche-e- } \\
\text { Commerciali; } \\
43(3), 1996, \mathrm{pp} . \\
517-36\end{array}$ & 1996 & Socher & Karl & $\begin{array}{r}\text { Universität } \\
\text { Innsbruck }\end{array}$ & 0 & 1 & 2 & 0 & K. Socher: 0 \\
\hline
\end{tabular}




\begin{tabular}{|c|c|c|c|c|c|c|c|c|c|c|c|}
\hline $\begin{array}{r}\text { Composite } \\
\text { Commodities and the } \\
\text { Prebisch-Singer } \\
\text { Thesis }\end{array}$ & $\begin{array}{r}\text { Chen,-John- } \\
\text { Ren; Stocker,- } \\
\text { Herbert }\end{array}$ & $\begin{array}{r}\text { World- } \\
\text { Development; } \\
\text { 25(11), November } \\
\text { 1997, pages 1863- } \\
71 .\end{array}$ & 1997 & Stocker & Herbert & $\begin{array}{r}\text { Universität } \\
\text { Innsbruck }\end{array}$ & 0,76 & 0,5 & 2 & $\begin{array}{r}0,25333333 \\
3\end{array}$ & $\begin{array}{r}\text { H. Stocker: } \\
0.25333\end{array}$ \\
\hline $\begin{array}{r}\text { Some Remarks on } \\
\text { the "System of } \\
\text { Integrated } \\
\text { Environmental and } \\
\text { Economic } \\
\text { Accounting" of the } \\
\text { United Nations }\end{array}$ & $\begin{array}{r}\text { Holub,-Hans- } \\
\text { Werner; } \\
\text { Tappeiner,- } \\
\text { Gottfried; } \\
\text { Tappeiner,- } \\
\text { Ulrike }\end{array}$ & $\begin{array}{r}\text { Ecological- } \\
\text { Economics; 29(3), } \\
\text { June 1999, pages } \\
329-36 .\end{array}$ & 1999 & Tappeiner & Gottfried & $\begin{array}{r}\text { Universität } \\
\text { Innsbruck }\end{array}$ & 0,64 & 0,5 & 3 & 0,16 & \\
\hline $\begin{array}{l}\text { Modeling on the } \\
\text { Basis of Models }\end{array}$ & $\begin{array}{r}\text { Holub,-Hans- } \\
\text { Werner; } \\
\text { Tappeiner,- } \\
\text { Gottfried }\end{array}$ & $\begin{array}{r}\text { Review-of-Income- } \\
\text { and-Wealth; 43(4), } \\
\text { December 1997, } \\
\text { pages 505-10. }\end{array}$ & 1997 & Tappeiner & Gottfried & $\begin{array}{r}\text { Universität } \\
\text { Innsbruck }\end{array}$ & 0,17 & 0,5 & 2 & $\begin{array}{r}0,05666666 \\
7\end{array}$ & \\
\hline $\begin{array}{r}\text { Ex post, ex ante und } \\
\text { dazwischen. } \\
\text { Uberlegungen zu } \\
\text { einem zu wenig } \\
\text { beachteten Problem } \\
\text { der Empirischen } \\
\text { Wirtschaftsforschung }\end{array}$ & $\begin{array}{r}\text { Holub,-Hans- } \\
\text { Werner; } \\
\text { Tappeiner,- } \\
\text { Gottfried }\end{array}$ & $\begin{array}{r}\text { Jahrbucher-fur- } \\
\text { Nationalokonomie- } \\
\text { und-Statistik; } \\
\text { 214(6), Nov. 1995, } \\
\text { pages 663-74. }\end{array}$ & 1995 & Tappeiner & Gottfried & $\begin{array}{r}\text { Universität } \\
\text { Innsbruck }\end{array}$ & 0,21 & 1 & 2 & 0,14 & \\
\hline $\begin{array}{l}\text { The Iron Law of } \\
\text { Important Articles }\end{array}$ & $\begin{array}{r}\text { Holub,-Hans- } \\
\text { Werner; } \\
\text { Tappeiner,- } \\
\text { Gottfried.; } \\
\text { Eberharter,- } \\
\text { Veronika }\end{array}$ & $\begin{array}{r}\text { Southern- } \\
\text { Economic-Journal; } \\
58(2) \text {, October } \\
\text { 1991, pages 317- } \\
28 .\end{array}$ & 1991 & Tappeiner & Gottfried & \begin{tabular}{r|} 
Universität \\
Innsbruck
\end{tabular} & 0,39 & 1 & 3 & 0,195 & G. Tappeiner: \\
\hline $\begin{array}{r}\text { Zum Erkenntniswert } \\
\text { der zeitgenossischen } \\
\text { Dogmengeschichts- } \\
\text { schreibung. Eine } \\
\text { quantitative } \\
\text { Untersuchung } \\
\text { anhand ... }\end{array}$ & $\begin{array}{r}\text { Holub,-Hans- } \\
\text { Werner; } \\
\text { Kohler,- } \\
\text { Andreas; } \\
\text { Tappeiner,- } \\
\text { Gottfried. }\end{array}$ & $\begin{array}{r}\text { Jahrbucher-fur- } \\
\text { Nationalokonomie- } \\
\text { und-Statistik; } \\
\text { 208(5), Sept. 1991, } \\
\text { pages 525-34. }\end{array}$ & 1991 & Tappeiner & Gottfried & \begin{tabular}{r|} 
Universität \\
Innsbruck
\end{tabular} & 0,21 & 0,5 & 3 & 0,0525 & 0,604166667 \\
\hline $\begin{array}{r}\text { The Economics of } \\
\text { Credit Information }\end{array}$ & $\begin{array}{r}\text { Chen,-John- } \\
\text { ren }\end{array}$ & $\begin{array}{r}\text { Zagreb- } \\
\text { International- } \\
\text { Review-of- }\end{array}$ & 1999 & Chen & John-Ren & \begin{tabular}{r|} 
Universität \\
Innsbruck
\end{tabular} & 0 & 1 & 1 & 0 & \\
\hline
\end{tabular}




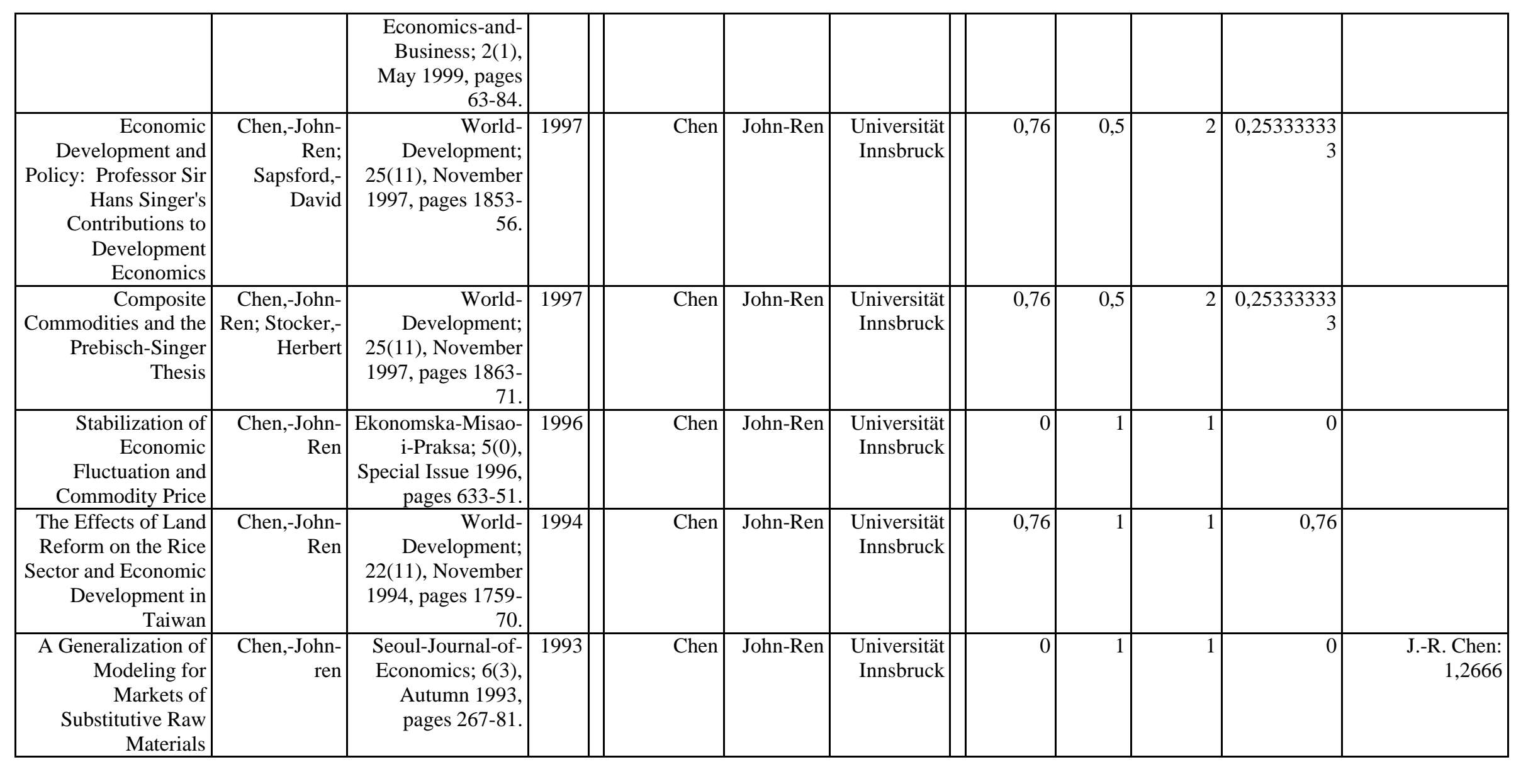

Average: 


\begin{tabular}{|c|c|c|c|c|c|c|c|c|c|c|}
\hline & Author(s) & Journal & Year & & & & $\begin{array}{l}\text { Impact } \\
\text { Factor }\end{array}$ & $\begin{array}{c}\text { Seiten- } \\
\text { korr. }\end{array}$ & Autoren & Impact \\
\hline $\begin{array}{l}\text { Policymakers' Preferences and the Optimal } \\
\text { Budget for Austria }\end{array}$ & $\begin{array}{l}\text { Neck,-Reinhard; } \\
\text { Karbuz,-Sohbet }\end{array}$ & \begin{tabular}{|l} 
International-Advances-in- \\
Economic-Research; 2(3), \\
Aug. 1996, pp. 314-23. \\
\end{tabular} & 1996 & Neck & Reinhard & \begin{tabular}{|l} 
Universität \\
Klagenfurt
\end{tabular} & 0 & 0.5 & 2 & 0 \\
\hline $\begin{array}{l}\text { What Remains of the Popularity Function? } \\
\text { The Case of Austria }\end{array}$ & $\begin{array}{l}\text { Neck,-Reinhard; } \\
\text { Karbuz,-Sohbet }\end{array}$ & $\begin{array}{l}\text { International-Advances-in- } \\
\text { Economic-Research; 1(3), } \\
\text { Aug. 1995, pp. 263-71. } \\
\end{array}$ & 1995 & Neck & Reinhard & $\begin{array}{l}\text { Universität } \\
\text { Klagenfurt }\end{array}$ & 0 & 0.5 & 2 & 0 \\
\hline $\begin{array}{l}\text { Econometric Estimations of Popularity } \\
\text { Functions: A Case Study for Austria }\end{array}$ & $\begin{array}{l}\text { Neck,-Reinhard; } \\
\text { Karbuz,-Sohbet }\end{array}$ & $\begin{array}{l}\text { Public-Choice; 91(1), April } \\
\text { 1997, pp. 57-88. }\end{array}$ & 1997 & Neck & Reinhard & \begin{tabular}{|l|} 
Universität \\
Klagenfurt \\
\end{tabular} & 0.43 & 1 & 2 & 0.2866666667 \\
\hline $\begin{array}{l}\text { Commitment and Coordination in a Dynamic } \\
\text { Game Model of International Economic Policy- } \\
\text { Making }\end{array}$ & \begin{tabular}{|l|} 
Neck,-Reinhard; \\
Dockner,-Engelbert-J.
\end{tabular} & $\begin{array}{l}\text { Open-Economies-Review; } \\
\text { 6(1), January 1995, } \\
\text { pp. 5-28. }\end{array}$ & 1995 & Neck & Reinhard & \begin{tabular}{|l} 
Universität \\
Klagenfurt
\end{tabular} & 0 & 1 & 2 & 0 \\
\hline
\end{tabular}


Universität Mannheim

\begin{tabular}{|c|c|c|c|c|c|c|c|c|c|c|c|}
\hline & Author(s) & Journal & Year & & & & $\begin{array}{l}\text { Impact } \\
\text { Factor }\end{array}$ & $\begin{array}{l}\text { Page- } \\
\text { corr. }\end{array}$ & Authors & Impact & personal Impact \\
\hline $\begin{array}{l}\text { Anonymous Games with Binary } \\
\text { Actions }\end{array}$ & Blonski,-Matthias & $\begin{array}{l}\text { Games-and-Economic- } \\
\text { Behavior; } 28(2), \text { August } \\
\text { 1999, pp. 171-80. }\end{array}$ & 1999 & Blonski & Matthias & U Mannheim & 0.6 & 0.5 & 1 & 0.3 & \\
\hline $\begin{array}{l}\text { When Is Rational Behavior Consistent } \\
\text { with Rules of Thumb? A Link between } \\
\text { Evolutionary Terminology and } \\
\text { Neoclassical Methodology }\end{array}$ & Blonski,-Matthias & $\begin{array}{l}\text { Journal-of-Mathematical- } \\
\text { Economics; } 32(1) \text {, August } \\
\text { 1999, pp. 131-44. }\end{array}$ & 1999 & Blonski & Matthias & U Mannheim & 0.37 & 1 & 1 & 0.37 & \\
\hline $\begin{array}{l}\text { Social Learning with Case-Based } \\
\text { Decisions }\end{array}$ & Blonski,-Matthias & $\begin{array}{l}\text { Journal-of-Economic- } \\
\text { Behavior-and-Organization; } \\
\text { 38(1), Jan. 1999, pp. 59- } \\
77 .\end{array}$ & 1999 & Blonski & Matthias & U Mannheim & 0.42 & 1 & 1 & 0.42 & \\
\hline $\begin{array}{l}\text { Innovation and Evolution as a Dynamic } \\
\text { Game with Rational Behaviour }\end{array}$ & Blonski,-Matthias & \begin{tabular}{l|} 
Empirica; 20(2), 1993, \\
pp. 147-58.
\end{tabular} & 1993 & Blonski & Matthias & U Mannheim & 0 & 1 & 1 & 0 & M. Blonski: 1.09 \\
\hline $\begin{array}{l}\text { Entwicklungspolitische Spekulationen } \\
\text { uber die Industrielle Revolution in } \\
\text { Grossbritannien. }\end{array}$ & $\begin{array}{l}\text { Buchheim,-- } \\
\text { Christoph }\end{array}$ & $\begin{array}{l}\text { Ifo-Studien; 38(2), 1992, } \\
\text { pp. 133-54. }\end{array}$ & 1992 & Buchheim & Christoph & U Mannheim & 0 & 1 & 1 & 0 & C. Buchheim: 0 \\
\hline $\begin{array}{l}\text { Resource and Waste Taxation in the } \\
\text { Theory of the Firm with Recycling } \\
\text { Activities }\end{array}$ & Conrad,-Klaus & $\begin{array}{l}\text { Environmental-and- } \\
\text { Resource-Economics; } \\
\text { 14(2), September 1999, } \\
\text { pp. 217-42. }\end{array}$ & 1999 & Conrad & Klaus & U Mannheim & 0 & 1 & 1 & 0 & \\
\hline $\begin{array}{l}\text { Economic Effects of an Uncoordinated } \\
\text { versus a Coordinated Carbon Dioxide } \\
\text { Policy in the European Union: An } \\
\text { Applied General ... }\end{array}$ & $\begin{array}{l}\text { Conrad,-Klaus; } \\
\text { Schmidt,-Tobias- } \\
\text { F.-N. } \\
\end{array}$ & $\begin{array}{l}\text { Economic-Systems - } \\
\text { Research; 10(2), June } \\
\text { 1998, pp. 161-82. }\end{array}$ & 1998 & Conrad & Klaus & U Mannheim & 0 & 1 & 2 & 0 & \\
\hline \begin{tabular}{|l|} 
Infrastructure Provision and \\
International Market Share Rivalry
\end{tabular} & $\begin{array}{l}\text { Conrad,-Klaus; } \\
\text { Seitz,-Helmut }\end{array}$ & $\begin{array}{l}\text { Regional-Science-and- } \\
\text { Urban-Economics; } 27(6), \\
\text { November 1997, pp. } 715- \\
34 . \\
\end{array}$ & 1997 & Conrad & Klaus & U Mannheim & 0.56 & 1 & 2 & 0.373333333 & \\
\hline $\begin{array}{l}\text { Traffic, Transportation, Infrastructure } \\
\text { and Externalities: A Theoretical } \\
\text { Framework for a CGE Analysis }\end{array}$ & Conrad,-Klaus & $\begin{array}{l}\text { Annals-of-Regional- } \\
\text { Science; 31(4), September } \\
\text { 1997, pp. 369-89. }\end{array}$ & 1997 & Conrad & Klaus & U Mannheim & 0.22 & 1 & 1 & 0.22 & \\
\hline $\begin{array}{l}\text { Der Beitrag von } \\
\text { Fertigungsorganisation, Infrastruktur } \\
\text { und FuE im Aufholprozess der } \\
\text { japanischen Elektrotechnik- und } \\
\text { Strassenfahrzeug... }\end{array}$ & $\begin{array}{l}\text { Conrad,-Klaus; } \\
\text { Wastl,-Dieter }\end{array}$ & $\begin{array}{l}\text { Ifo-Studien; 41(3), 1995, } \\
\text { pp. 355-80. }\end{array}$ & 1995 & Conrad & Klaus & U Mannheim & 0 & 1 & 2 & 0 & \\
\hline $\begin{array}{l}\text { The Impact of Environmental } \\
\text { Regulation on Productivity in German } \\
\text { Industries }\end{array}$ & $\begin{array}{l}\text { Conrad,-Klaus; } \\
\text { Wastl,-Dieter }\end{array}$ & $\begin{array}{l}\text { Empirical-Economics; } \\
\text { 20(4), 1995, pp. 615-33. }\end{array}$ & 1995 & Conrad & Klaus & U Mannheim & 0 & 1 & 2 & 0 & \\
\hline $\begin{array}{l}\text { The Control of CO2 Emissions and Its } \\
\text { Economic Impact: An AGE Model for } \\
\text { a German State }\end{array}$ & $\begin{array}{l}\text { Conrad,-Klaus; } \\
\text { Schroder,-Michael }\end{array}$ & \begin{tabular}{l|} 
Environmental-and- \\
Resource-Economics; 1(3), \\
1991, pp. 289-312.
\end{tabular} & 1991 & Conrad & Klaus & U Mannheim & 0 & 1 & 2 & 0 & \\
\hline $\begin{array}{l}\text { On the Design of Incentive } \\
\text { Mechanisms in Environmental Policy }\end{array}$ & $\begin{array}{l}\text { Conrad,-Klaus; } \\
\text { Wang,-Jianmin }\end{array}$ & $\begin{array}{l}\text { Environmental-and- } \\
\text { Resource-Economics; 3(3), } \\
\text { June 1993, pp. 245-62. }\end{array}$ & 1993 & Conrad & Klaus & U Mannheim & 0 & 1 & 2 & 0 & \\
\hline $\begin{array}{l}\text { The Economic Benefits of Public } \\
\text { Infrastructure }\end{array}$ & $\begin{array}{l}\text { Conrad,-Klaus; } \\
\text { Seitz,-Helmut }\end{array}$ & $\begin{array}{l}\text { Applied-Economics; 26(4), } \\
\text { April 1994, pp. 303-11. }\end{array}$ & 1994 & Conrad & Klaus & U Mannheim & 0.28 & 1 & 2 & 0.186666667 & \\
\hline Choosing Environmental Policy & Conrad,-Klaus; & Journal-of-Policy-Modeling; & 1993 & Conrad & Klaus & U Mannheim & 0.12 & 1 & 2 & 0.08 & \\
\hline
\end{tabular}


Universität Mannheim

\begin{tabular}{|c|c|c|c|c|c|c|c|c|c|c|c|}
\hline & Author(s) & Journal & Year & & & & \begin{tabular}{c|} 
Impact \\
Factor
\end{tabular} & $\begin{array}{l}\text { Page- } \\
\text { corr. }\end{array}$ & Authors & Impact & personal Impact \\
\hline $\begin{array}{l}\text { Instruments Using General Equilibrium } \\
\text { Models }\end{array}$ & Schroder,-Michael & $\begin{array}{l}\text { 15(5-6), Oct.-Dec. 1993, } \\
\text { pp. 521-43. }\end{array}$ & & & & & & & & & \\
\hline $\begin{array}{l}\text { Quantitative Umweltpolitik: } \\
\text { Gesamtwirtschaftliche Auswirkungen } \\
\text { einer CO2-Besteuerung in } \\
\text { Deutschland (West). }\end{array}$ & $\begin{array}{l}\text { Conrad,-Klaus; } \\
\text { Wang,-Jianmin }\end{array}$ & $\begin{array}{l}\text { Jahrbucher-fur- } \\
\text { Nationalokonomie-und- } \\
\text { Statistik; 212(3-4), Sept. } \\
\text { 1993, pp. 309-24. }\end{array}$ & 1993 & Conrad & Klaus & U Mannheim & 0.21 & 1 & 2 & 0.14 & \\
\hline $\begin{array}{l}\text { The Effect of Emission Taxes and } \\
\text { Abatement Subsidies on Market } \\
\text { Structure }\end{array}$ & $\begin{array}{l}\text { Conrad,-Klaus; } \\
\text { Wang,-Jianmin }\end{array}$ & $\begin{array}{l}\text { International-Journal-of- } \\
\text { Industrial-Organization; } \\
11(4), 1993 \text {, pp. 499-518. }\end{array}$ & 1993 & Conrad & Klaus & U Mannheim & 0.34 & 1 & 2 & 0.226666667 & \\
\hline $\begin{array}{l}\text { The Impact of Embodied and } \\
\text { Disembodied Technical Progress on } \\
\text { Productivity Gaps--An Applied General } \\
\text { Equilibrium Analysis for ... }\end{array}$ & $\begin{array}{l}\text { Conrad,-Klaus; } \\
\text { Ehrlich,-Martin } \\
\end{array}$ & $\begin{array}{l}\text { Journal-of-Productivity- } \\
\text { Analysis; 4(3), September } \\
\text { 1993, pp. 317-35. }\end{array}$ & 1993 & Conrad & Klaus & U Mannheim & 0 & 1 & 2 & 0 & \\
\hline \begin{tabular}{l|} 
Taxes and Subsidies for Pollution- \\
Intensive Industries as Trade Policy
\end{tabular} & Conrad,-Klaus & $\begin{array}{l}\text { Journal-of-Environmental- } \\
\text { Economics-and- } \\
\text { Management; } 25(2) \text {, Sept. } \\
\text { 1993, pp. 121-35. }\end{array}$ & 1993 & Conrad & Klaus & U Mannheim & 1.41 & 1 & 1 & 1.41 & \\
\hline $\begin{array}{l}\text { Investment in Education and U.S. } \\
\text { Economic Growth: Comment }\end{array}$ & Conrad,-Klaus & $\begin{array}{l}\text { Scandinavian-Journal-of- } \\
\text { Economics; 94(0), } \\
\text { Supplement 1992, pp. S71- } \\
74 \text {. }\end{array}$ & 1992 & Conrad & Klaus & U Mannheim & 0.48 & 0.5 & 1 & 0.24 & \\
\hline $\begin{array}{l}\text { The "Public Capital Hypothesis": The } \\
\text { Case of Germany }\end{array}$ & $\begin{array}{l}\text { Conrad,-Klaus; } \\
\text { Seitz,-Helmut }\end{array}$ & $\begin{array}{l}\text { Recherches-Economiques- } \\
\text { de-Louvain; 58(3-4), 1992, } \\
\text { pp. 309-27. }\end{array}$ & 1992 & Conrad & Klaus & U Mannheim & 0 & 1 & 2 & 0 & \\
\hline $\begin{array}{l}\text { Allokations- und Wohlfahrtseffekte der } \\
\text { Besteuerung: Die Vorschlage zur } \\
\text { Reform der } \\
\text { Unternehmensbesteuerung. }\end{array}$ & Conrad,-Klaus & $\begin{array}{l}\text { Kredit-und-Kapital; 25(3), } \\
\text { 1992, pp. 359-85. }\end{array}$ & 1992 & Conrad & Klaus & U Mannheim & 0 & 1 & 1 & 0 & \\
\hline $\begin{array}{l}\text { An Evaluation of Taxes on Air } \\
\text { Pollutants Emissions: An Applied } \\
\text { General Equilibrium Approach }\end{array}$ & \begin{tabular}{|l|} 
Conrad,-Klaus; \\
Schroder,-Michael
\end{tabular} & $\begin{array}{l}\text { Schweizerische-Zeitschrift- } \\
\text { fur-Volkswirtschaft- } \\
\text { undStatistik; 127(2), 1991, } \\
\text { pp. 199-224. }\end{array}$ & 1991 & Conrad & Klaus & U Mannheim & 0 & 1 & 2 & 0 & \\
\hline A Test for Strategic Excess Capacity & $\begin{array}{l}\text { Conrad,-Klaus; } \\
\text { Veall,-Michael-R. }\end{array}$ & $\begin{array}{l}\text { Empirical-Economics; } \\
\text { 16(4), 1991, pp. 433-45. }\end{array}$ & 1991 & Conrad & Klaus & U Mannheim & 0 & 1 & 2 & 0 & \\
\hline $\begin{array}{l}\text { Incentive Mechanisms for } \\
\text { Environmental Protection under } \\
\text { Asymmetric Information: A Case } \\
\text { Study }\end{array}$ & Conrad,-Klaus & $\begin{array}{l}\text { Applied-Economics; 23(5), } \\
\text { May 1991, pp. 871-80. }\end{array}$ & 1991 & Conrad & Klaus & U Mannheim & 0.28 & 0.5 & 1 & 0.14 & \\
\hline $\begin{array}{l}\text { Taxes on Emissions, Conjectural } \\
\text { Variations and Overinvestment in } \\
\text { Abatement Capital }\end{array}$ & Conrad,-Klaus & $\begin{array}{l}\text { Journal-of-Institutional-and- } \\
\text { Theoretical-Economics; } \\
\text { 146(2), June 1990, } \\
\text { pp. 281-97. }\end{array}$ & 1990 & Conrad & Klaus & U Mannheim & 0.29 & 1 & 1 & 0.29 & $\begin{array}{l}\text { K. Conrad: } \\
\text { 3.306666667 }\end{array}$ \\
\hline $\begin{array}{l}\text { Microeconometric Models of Tourists' } \\
\text { Destination Choice }\end{array}$ & $\begin{array}{l}\text { Eymann,-- } \\
\text { Angelika; } \\
\text { Ronning,-Gerd }\end{array}$ & $\begin{array}{l}\text { Regional-Science-and- } \\
\text { Urban-Economics; } 27(6), \\
\text { November 1997, pp. } 735- \\
61 .\end{array}$ & 1997 & Eymann & Angelika & U Mannheim & 0.56 & 1 & 2 & 0.373333333 & A. Eymann: \\
\hline $\begin{array}{l}\text { Antidumping Policy in the European } \\
\text { Community: Political Discretion or }\end{array}$ & $\begin{array}{l}\text { Eymann,-A.; } \\
\text { Schuknecht,-L. }\end{array}$ & $\begin{array}{l}\text { Economics-and-Politics; } \\
\text { 8(2), July 1996, pp. 111-31. }\end{array}$ & 1996 & Eymann & Angelika & U Mannheim & 0 & 1 & 2 & 0 & 0.373333333 \\
\hline
\end{tabular}


Universität Mannheim

\begin{tabular}{|c|c|c|c|c|c|c|c|c|c|c|c|}
\hline & Author(s) & Journal & Year & & & & $\begin{array}{l}\text { Impact } \\
\text { Factor }\end{array}$ & $\begin{array}{l}\text { Page- } \\
\text { corr. }\end{array}$ & Authors & Impact & personal Impact \\
\hline \multicolumn{12}{|l|}{ Technical Determination } \\
\hline $\begin{array}{l}\text { Wer ist noch Mitglied in } \\
\text { Gewerkschaften? Eine Panelanalyse } \\
\text { fur Westdeutschland. }\end{array}$ & $\begin{array}{l}\text { Fitzenberger,-B.; } \\
\text { Haggeney,-I.; } \\
\text { Ernst,-M. }\end{array}$ & $\begin{array}{l}\text { Zeitschrift-fur-Wirtschafts-- } \\
\text { und-Sozialwissenschaften; } \\
\text { 119(2), 1999, pp. 223-63. }\end{array}$ & 1999 & Fitzenberger & Bernd & U Mannheim & 0 & 1 & 3 & 0 & \\
\hline $\begin{array}{l}\text { Industry-Level Wage Bargaining: A } \\
\text { Partial Rehabilitation---The German } \\
\text { Experience }\end{array}$ & $\begin{array}{l}\text { Fitzenberger,-- } \\
\text { Bernd; Franz,- } \\
\text { Wolfgang }\end{array}$ & \begin{tabular}{l|} 
Scottish-Journal-of- \\
Political-Economy; 46(4), \\
September 1999, pp. 437- \\
57.
\end{tabular} & 1999 & Fitzenberger & Bernd & U Mannheim & 0.46 & 1 & 2 & 0.306666667 & \\
\hline $\begin{array}{l}\text { International Trade and the Skill } \\
\text { Structure of Wages and Employment } \\
\text { in West Germany }\end{array}$ & $\begin{array}{l}\text { Fitzenberger,-- } \\
\text { Bernd }\end{array}$ & $\begin{array}{l}\text { Jahrbucher-fur- } \\
\text { Nationalokonomie-und- } \\
\text { Statistik; 219(1-2), July } \\
\text { 1999, pp. 67-89. } \\
\end{array}$ & 1999 & Fitzenberger & Bernd & U Mannheim & 0.21 & 1 & 1 & 0.21 & \\
\hline $\begin{array}{l}\text { Aussenhandel, technischer Fortschritt } \\
\text { und Arbeitsmarkt in Westdeutschland } \\
\text { von } 1975 \text { bis } 1990 \text {. }\end{array}$ & $\begin{array}{l}\text { Fitzenberger,-- } \\
\text { Bernd }\end{array}$ & $\begin{array}{l}\text { Mitteilungen-aus-der- } \\
\text { Arbeitsmarkt--und- } \\
\text { Berufsforschung; 30(3), } \\
\text { 1997, pp. 642-51. }\end{array}$ & 1997 & Fitzenberger & Bernd & U Mannheim & 0 & 1 & 1 & 0 & \\
\hline $\begin{array}{l}\text { The Moving Blocks Bootstrap and } \\
\text { Robust Inference for Linear Least } \\
\text { Squares and Quantile Regressions }\end{array}$ & $\begin{array}{l}\text { Fitzenberger,-- } \\
\text { Bernd }\end{array}$ & $\begin{array}{l}\text { Journal-of-Econometrics; } \\
\text { 82(2), February 1998, } \\
\text { pp. 235-87. }\end{array}$ & 1998 & Fitzenberger & Bernd & U Mannheim & 1.06 & 1 & 1 & 1.06 & \\
\hline $\begin{array}{l}\text { Assessing the Impact of Training on } \\
\text { Employment: The Case of East } \\
\text { Germany }\end{array}$ & $\begin{array}{l}\text { Fitzenberger,- } \\
\text { Bernd; Prey,- } \\
\text { Hedwig }\end{array}$ & $\begin{array}{l}\text { Ifo-Studien; 43(1), 1997, } \\
\text { pp. 71-116. }\end{array}$ & 1997 & Fitzenberger & Bernd & U Mannheim & 0 & 1 & 2 & 0 & $\begin{array}{l}\text { B. Fitzenberger: } \\
1.576666667\end{array}$ \\
\hline $\begin{array}{l}\text { Arbeitslosigkeit in Deutschland und } \\
\text { Moglichkeiten ihrer Uberwindung: Eine } \\
\text { Bestandsaufnahme und Analyse aus } \\
\text { der Sicht ... }\end{array}$ & Franz,-Wolfgang & $\begin{array}{l}\text { Empirica; 26(2), 1999, } \\
\text { pp. 151-67. }\end{array}$ & 1999 & Franz & Wolfgang & U Mannheim & 0 & 1 & 1 & 0 & \\
\hline $\begin{array}{l}\text { Industry-Level Wage Bargaining: A } \\
\text { Partial Rehabilitation--The German } \\
\text { Experience }\end{array}$ & $\begin{array}{l}\text { Fitzenberger,- } \\
\text { Bernd; Franz,- } \\
\text { Wolfgang }\end{array}$ & $\begin{array}{l}\text { Scottish-Journal-of- } \\
\text { Political-Economy; 46(4), } \\
\text { September 1999, pp. 437- } \\
57 .\end{array}$ & 1999 & Franz & Wolfgang & U Mannheim & 0.46 & 1 & 2 & 0.306666667 & \\
\hline $\begin{array}{l}\text { Mobilitat nach der beruflichen } \\
\text { Erstausbildung: Eine empirische } \\
\text { Studie fur Westdeutschland. }\end{array}$ & $\begin{array}{l}\text { Franz,-Wolfgang; } \\
\text { Zimmermann,-- } \\
\text { Volker }\end{array}$ & $\begin{array}{l}\text { Jahrbucher-fur- } \\
\text { Nationalokonomie-und- } \\
\text { Statistik; 219(1-2), July } \\
\text { 1999, pp. 143-64. }\end{array}$ & 1999 & Franz & Wolfgang & U Mannheim & 0.21 & 1 & 2 & 0.14 & \\
\hline $\begin{array}{l}\text { Einfuhrung zum Themenheft: } \\
\text { Lohnstruktur, Qualifikation und } \\
\text { Mobilitat }\end{array}$ & Franz,-Wolfgang & $\begin{array}{l}\text { Jahrbucher-fur- } \\
\text { Nationalokonomie-und- } \\
\text { Statistik; 219(1-2), July } \\
\text { 1999, pp. 1-7. }\end{array}$ & 1999 & Franz & Wolfgang & U Mannheim & 0.21 & 0.5 & 1 & 0.105 & \\
\hline $\begin{array}{l}\text { Valuing the Marital Portion in Defined } \\
\text { Benefit Plans in Dissolution Cases } \\
\text { Consistent with the Concept of } \\
\text { Community Property }\end{array}$ & $\begin{array}{l}\text { Franz,-Wolfgang- } \\
\text { W. }\end{array}$ & $\begin{array}{l}\text { Journal-of-Legal- } \\
\text { Economics; 6(3), Winter } \\
\text { 1996-1997, pp. 39-53. }\end{array}$ & 1996 & Franz & Wolfgang & U Mannheim & 0 & 1 & 1 & 0 & \\
\hline $\begin{array}{l}\text { Konjunkturpolitik: Erfahrungen und } \\
\text { Perspektiven }\end{array}$ & Franz,-Wolfgang & $\begin{array}{l}\text { Ifo-Studien; 43(2), 1997, } \\
\text { pp. 185-94. }\end{array}$ & 1997 & Franz & Wolfgang & U Mannheim & 0 & 0.5 & 1 & 0 & \\
\hline $\begin{array}{l}\text { Ist die Tarifautonomie noch } \\
\text { zeitgemass? }\end{array}$ & Franz,-Wolfgang & $\begin{array}{l}\text { Zeitschrift-fur- } \\
\text { Wirtschaftspolitik; 45(1), } \\
\text { 1996, pp. 31-38. }\end{array}$ & 1996 & Franz & Wolfgang & U Mannheim & 0 & 0.5 & 1 & 0 & \\
\hline The Meaning of "Hedonic Damages" in & Franz,-Wolfgang- & Journal-of-Forensic- & 1996 & Franz & Wolfgang & U Mannheim & 0 & 0.5 & 1 & 0 & \\
\hline
\end{tabular}


Universität Mannheim

\begin{tabular}{|c|c|c|c|c|c|c|c|c|c|c|c|}
\hline & Author(s) & Journal & Year & & & & \begin{tabular}{c|} 
Impact \\
Factor
\end{tabular} & $\begin{array}{l}\text { Page- } \\
\text { corr. }\end{array}$ & Authors & Impact & personal Impact \\
\hline Tort Litigation: A Note & W. & $\begin{array}{l}\text { Economics; 9(1), Winter } \\
\text { 1996, pp. 55-57. }\end{array}$ & & & & & & & & & \\
\hline $\begin{array}{l}\text { Sectoral Wage and Price Formation } \\
\text { and Working Time in Germany: An } \\
\text { Econometric Analysis }\end{array}$ & $\begin{array}{l}\text { Franz,-Wolfgang; } \\
\text { Smolny,-Werner }\end{array}$ & $\begin{array}{l}\text { Zeitschrift-fur-Wirtschafts-- } \\
\text { und-Sozialwissenschaften; } \\
\text { 114(4), 1994, pp. 507-29. }\end{array}$ & 1994 & Franz & Wolfgang & U Mannheim & 0 & 1 & 2 & 0 & \\
\hline $\begin{array}{l}\text { Gross Worker and Job Flows in } \\
\text { Europe: Comments }\end{array}$ & Franz,-Wolfgang & $\begin{array}{l}\text { European-Economic- } \\
\text { Review; 38(6), June 1994, } \\
\text { pp. 1321-25. }\end{array}$ & 1994 & Franz & Wolfgang & U Mannheim & 0.84 & 0.5 & 1 & 0.42 & \\
\hline $\begin{array}{l}\text { A Macroeconometric Disequilibrium } \\
\text { Analysis of Current and Future } \\
\text { Migration from Eastern Europe into } \\
\text { West Germany }\end{array}$ & $\begin{array}{l}\text { Franz,-W.; Oser,- } \\
\text { U.; Winker,-P. }\end{array}$ & $\begin{array}{l}\text { Journal-of-Population- } \\
\text { Economics; } 7(2), 1994, \\
\text { pp. 217-34. }\end{array}$ & 1994 & Franz & Wolfgang & U Mannheim & 0.44 & 1 & 3 & 0.22 & \\
\hline $\begin{array}{l}\text { Calculating the Present Value of } \\
\text { Future Fringe Benefits: What Net } \\
\text { Discount Rate to Use }\end{array}$ & $\begin{array}{l}\text { Franz,-W.-W.; } \\
\text { Maglietti,-F.; } \\
\text { Kalich,-C. }\end{array}$ & $\begin{array}{l}\text { Journal-of-Legal- } \\
\text { Economics; } 2(1), \text { March } \\
\text { 1992, pp. 55-66. }\end{array}$ & 1992 & Franz & Wolfgang & U Mannheim & 0 & 1 & 3 & 0 & \\
\hline $\begin{array}{l}\text { German and American Wage and } \\
\text { Price Dynamics: Differences and } \\
\text { Common Themes }\end{array}$ & $\begin{array}{l}\text { Franz,-Wolfgang; } \\
\text { Gordon,-Robert-J. }\end{array}$ & $\begin{array}{l}\text { European-Economic- } \\
\text { Review; 37(4), May 1993, } \\
\text { pp. 719-54. }\end{array}$ & 1993 & Franz & Wolfgang & U Mannheim & 0.84 & 1 & 2 & 0.56 & \\
\hline $\begin{array}{l}\text { Should Net Discount Rates Vary by } \\
\text { Level of Earnings? }\end{array}$ & $\begin{array}{l}\text { Franz,-Wolfgang- } \\
\text { W. }\end{array}$ & $\begin{array}{l}\text { Journal-of-Forensic- } \\
\text { Economics; 6(2), Spring- } \\
\text { Summer 1993, pp. 169-70. }\end{array}$ & 1993 & Franz & Wolfgang & U Mannheim & 0 & 0.5 & 1 & 0 & \\
\hline $\begin{array}{l}\text { The Effect of Recent Income Tax } \\
\text { Reforms on the Calculation of Lost } \\
\text { Earnings: A Reply }\end{array}$ & $\begin{array}{l}\text { Franz,-Wolfgang- } \\
\text { W. }\end{array}$ & $\begin{array}{l}\text { Journal-of-Forensic- } \\
\text { Economics; 6(1), Winter } \\
\text { 1992, pp. 61-62. }\end{array}$ & 1992 & Franz & Wolfgang & U Mannheim & 0 & 0.5 & 1 & 0 & \\
\hline $\begin{array}{l}\text { The Importance of Rationing in } \\
\text { International Trade: An Econometric } \\
\text { Analysis for Germany }\end{array}$ & $\begin{array}{l}\text { Franz,-Wolfgang; } \\
\text { Heidbrink,-Gustav }\end{array}$ & $\begin{array}{l}\text { Recherches-Economiques- } \\
\text { de-Louvain; 58(3-4), 1992, } \\
\text { pp. 347-71. }\end{array}$ & 1992 & Franz & Wolfgang & U Mannheim & 0 & 1 & 2 & 0 & \\
\hline $\begin{array}{l}\text { Eine Schatzung der inflationsstabilen } \\
\text { Arbeitslosenquote mit Hilfe von } \\
\text { Preiserwartungen des Ifo- } \\
\text { Sonjunkturtestes. }\end{array}$ & $\begin{array}{l}\text { Franz,-Wolfgang; } \\
\text { Hofmann,-Thomas }\end{array}$ & $\begin{array}{l}\text { Ifo-Studien; 36(4), January } \\
\text { 1990, pp. 211-27. }\end{array}$ & 1990 & Franz & Wolfgang & U Mannheim & 0 & 1 & 2 & 0 & \\
\hline $\begin{array}{l}\text { Hysteresis in Economic Relationships: } \\
\text { An Overview }\end{array}$ & Franz,-Wolfgang & $\begin{array}{l}\text { Empirical-Economics; } \\
15(2), 1990 \text {, pp. } 109-25 .\end{array}$ & 1990 & Franz & Wolfgang & U Mannheim & 0 & 1 & 1 & 0 & \\
\hline $\begin{array}{l}\text { Fiscal Policy in the Federal Republic of } \\
\text { Germany }\end{array}$ & Franz,-Wolfgang & $\begin{array}{l}\text { Empirical-Economics; } \\
15(1), 1990, \text { pp. } 17-54 .\end{array}$ & 1990 & Franz & Wolfgang & U Mannheim & 0 & 1 & 1 & 0 & \\
\hline $\begin{array}{l}\text { A Disequilibrium Approach to } \\
\text { Unemployment in the Federal Republic } \\
\text { of Germany }\end{array}$ & $\begin{array}{l}\text { Franz,-Wolfgang; } \\
\text { Konig,-Heinz }\end{array}$ & $\begin{array}{l}\text { European-Economic- } \\
\text { Review; 34(2-3), May } \\
\text { 1990, pp. 413-22. }\end{array}$ & 1990 & Franz & Wolfgang & U Mannheim & 0.84 & 0.5 & 2 & 0.28 & $\begin{array}{l}\text { W. Franz: } \\
2.031666667\end{array}$ \\
\hline $\begin{array}{l}\text { Discussion on International Contagion: } \\
\text { What Is It and What Can Be Done } \\
\text { Against It? }\end{array}$ & Hellwig,-Martin & $\begin{array}{l}\text { Schweizerische-Zeitschrift- } \\
\text { fur-Volkswirtschaft- } \\
\text { undStatistik; 134(4.2), Dec. } \\
\text { 1998, pp. 715-21. }\end{array}$ & 1998 & Hellwig & Martin & U Mannheim & 0 & 0.5 & 1 & 0 & \\
\hline $\begin{array}{l}\text { Banks, Markets, and the Allocation of } \\
\text { Risks in an Economy }\end{array}$ & Hellwig,-Martin & $\begin{array}{l}\text { Journal-of-Institutional-and- } \\
\text { Theoretical-Economics; } \\
\text { 154(1), March 1998, } \\
\text { pp. 328-45. }\end{array}$ & 1998 & Hellwig & Martin & U Mannheim & 0.29 & 1 & 1 & 0.29 & \\
\hline Capital Adequacy Rules as & Hellwig,-Martin & Schweizerische-Zeitschrift- & 1996 & Hellwig & Martin & U Mannheim & 0 & 0.5 & 1 & 0 & \\
\hline
\end{tabular}


Universität Mannheim

\begin{tabular}{|c|c|c|c|c|c|c|c|c|c|c|c|}
\hline & Author(s) & Journal & Year & & & & $\begin{array}{l}\text { Impact } \\
\text { Factor }\end{array}$ & $\begin{array}{l}\text { Page- } \\
\text { corr. }\end{array}$ & Authors & Impact & personal Impact \\
\hline $\begin{array}{l}\text { Instruments for the Regulation of } \\
\text { Banks: Introduction }\end{array}$ & & $\begin{array}{l}\text { fur-Volkswirtschaft- } \\
\text { undStatistik; 132(4/2), Dec. } \\
\text { 1996, pp. 609-11. }\end{array}$ & & & & & & & & & \\
\hline \begin{tabular}{l|} 
Rational Expectations Equilibria in \\
Sequence Economies with Symmetric \\
Information: The Two-Period Case
\end{tabular} & Hellwig,-Martin-F. & $\begin{array}{l}\text { Journal-of-Mathematical- } \\
\text { Economics; } 26(1), 1996, \\
\text { pp. 9-49. }\end{array}$ & 1996 & Hellwig & Martin & U Basel & 0.37 & 1 & 1 & 0.37 & \\
\hline $\begin{array}{l}\text { Sequential Decisions under } \\
\text { Uncertainty and the Maximum } \\
\text { Theorem }\end{array}$ & Hellwig,-Martin-F. & $\begin{array}{l}\text { Journal-of-Mathematical- } \\
\text { Economics; 25(4), May } \\
\text { 1996, pp. 443-64. }\end{array}$ & 1996 & Hellwig & Martin & U Basel & 0.37 & 1 & 1 & 0.37 & \\
\hline $\begin{array}{l}\text { The Assessment of Large Compounds } \\
\text { of Independent Gambles }\end{array}$ & Hellwig,-Martin-F. & $\begin{array}{l}\text { Journal-of-Economic- } \\
\text { Theory; 67(2), December } \\
\text { 1995, pp. 299-326. }\end{array}$ & 1995 & Hellwig & Martin & U Basel & 0.93 & 1 & 1 & 0.93 & \\
\hline $\begin{array}{l}\text { Systemic Aspects of Risk } \\
\text { Management in Banking and Finance }\end{array}$ & Hellwig,-Martin-F. & $\begin{array}{l}\text { Schweizerische-Zeitschrift- } \\
\text { fur-Volkswirtschaft- } \\
\text { undStatistik; 131(4/2), Dec. } \\
\text { 1995, pp. 723-37. }\end{array}$ & 1995 & Hellwig & Martin & U Basel & 0 & 1 & 1 & 0 & \\
\hline $\begin{array}{l}\text { The Macroeconomic Implications of } \\
\text { Capital Adequacy Requirements for } \\
\text { Banks }\end{array}$ & $\begin{array}{l}\text { Blum,-Jurg; } \\
\text { Hellwig,-Martin }\end{array}$ & \begin{tabular}{|l|} 
European-Economic- \\
Review; 39(3-4), April \\
1995, pp. 739-49.
\end{tabular} & 1995 & Hellwig & Martin & U Basel & 0.84 & 0.5 & 2 & 0.28 & \\
\hline $\begin{array}{l}\text { Liquidity Provision, Banking, and the } \\
\text { Allocation of Interest Rate Risk }\end{array}$ & Hellwig,-Martin & $\begin{array}{l}\text { European-Economic- } \\
\text { Review; 38(7), August } \\
\text { 1994, pp. 1363-89. } \\
\end{array}$ & 1994 & Hellwig & Martin & U Basel & 0.84 & 1 & 1 & 0.84 & \\
\hline The Challenge of Monetary Theory & Hellwig,-Martin-F. & $\begin{array}{l}\text { European-Economic- } \\
\text { Review; 37(2-3), April } \\
\text { 1993, pp. 215-42. }\end{array}$ & 1993 & Hellwig & Martin & U Basel & 0.84 & 1 & 1 & 0.84 & \\
\hline $\begin{array}{l}\text { Bertrand-Edgeworth Duopoly with } \\
\text { Proportional Residual Demand }\end{array}$ & $\begin{array}{l}\text { Allen,-Beth; } \\
\text { Hellwig,-Martin }\end{array}$ & $\begin{array}{l}\text { International-Economic- } \\
\text { Review; 34(1), February } \\
\text { 1993, pp. 39-60. } \\
\end{array}$ & 1993 & Hellwig & Martin & U Basel & 0.69 & 1 & 2 & 0.46 & \\
\hline $\begin{array}{l}\text { Fully Revealing Outcomes in } \\
\text { Signalling Models: An Example of } \\
\text { Nonexistence When the Type Space Is } \\
\text { Unbounded }\end{array}$ & Hellwig,-Martin & $\begin{array}{l}\text { Journal-of-Economic- } \\
\text { Theory; 58(1), October } \\
\text { 1992, pp. 93-104. }\end{array}$ & 1992 & Hellwig & Martin & U Basel & 0.93 & 1 & 1 & 0.93 & \\
\hline $\begin{array}{l}\text { The Marshall Plan: Economic Effects } \\
\text { and Implications for Eastern Europe } \\
\text { and the Former USSR: Discussion }\end{array}$ & Hellwig,-Martin & \begin{tabular}{l|} 
Economic-Policy:-A- \\
European-Forum; $0(14)$, \\
April 1992, pp. 55-58.
\end{tabular} & 1992 & Hellwig & Martin & U Basel & 0 & 0.5 & 1 & 0 & \\
\hline $\begin{array}{l}\text { Die Kommunikationsfunktion der } \\
\text { Finanzmarkte. }\end{array}$ & Hellwig,-Martin & $\begin{array}{l}\text { Schweizerische-Zeitschrift- } \\
\text { fur-Volkswirtschaft- } \\
\text { undStatistik; } 127(3), \text { Sept. } \\
\text { 1991, pp. 351-64. }\end{array}$ & 1991 & Hellwig & Martin & U Basel & 0 & 1 & 1 & 0 & \\
\hline $\begin{array}{l}\text { Subgame Perfect Equilibrium in } \\
\text { Continuous Games of Perfect } \\
\text { Information: An Elementary Approach } \\
\text { to Existence ... }\end{array}$ & $\begin{array}{l}\text { Hellwig,-M., } \\
\text { Leininger,-W., } \\
\text { Reny,-P., } \\
\text { Robson,-A. }\end{array}$ & $\begin{array}{l}\text { Journal-of-Economic- } \\
\text { Theory; } 52(2), \text { December } \\
\text { 1990, pp. 406-22. }\end{array}$ & 1990 & Hellwig & Martin & U Basel & 0.93 & 1 & 4 & 0.372 & M. Hellwig: 5.682 \\
\hline $\begin{array}{l}\text { Precommitment in Competing Vertical } \\
\text { Chains }\end{array}$ & Irmen,-Andreas & \begin{tabular}{|l|} 
Journal-of-Economic- \\
Surveys; $12(4)$, September \\
1998, pp. 333-59.
\end{tabular} & 1998 & Irmen & Andreas & U Mannheim & 0 & 1 & 1 & 0 & \\
\hline $\begin{array}{l}\text { Competition in Multi-characteristics } \\
\text { Spaces: Hotelling Was Almost Right }\end{array}$ & $\begin{array}{l}\text { Irmen,-A.; Thisse,-- } \\
\text { J.-F. }\end{array}$ & \begin{tabular}{|l|} 
Journal-of-Economic- \\
Theory; $78(1)$, January
\end{tabular} & 1998 & Irmen & Andreas & U Mannheim & 0.93 & 1 & 2 & 0.62 & \\
\hline
\end{tabular}


Universität Mannheim

\begin{tabular}{|c|c|c|c|c|c|c|c|c|c|c|c|}
\hline & Author(s) & Journal & Year & & & & $\begin{array}{l}\text { Impact } \\
\text { Factor }\end{array}$ & $\begin{array}{l}\text { Page- } \\
\text { corr. }\end{array}$ & Authors & Impact & personal Impact \\
\hline & & 1998, pp. 76-102. & & & & & & & & & \\
\hline Note on Duopolistic Vertical Restraints & Irmen,-Andreas & $\begin{array}{l}\text { European-Economic- } \\
\text { Review; 41(8), August } \\
\text { 1997, pp. 1559-67. }\end{array}$ & 1997 & Irmen & Andreas & U Mannheim & 0.84 & 0.5 & 1 & 0.42 & \\
\hline $\begin{array}{l}\text { Mark-Up Pricing and Bilateral } \\
\text { Monopoly }\end{array}$ & Irmen,-Andreas & $\begin{array}{l}\text { Economics-Letters; } 54(2) \\
\text { February 1997, pp. 179-84. }\end{array}$ & 1997 & Irmen & Andreas & U Mannheim & 0.26 & 0.5 & 1 & 0.13 & A. Irmen: 1.17 \\
\hline $\begin{array}{l}\text { Performance Measurement in Multi- } \\
\text { Period Agencies: Comment }\end{array}$ & Laux,-Christian & $\begin{array}{l}\text { Journal-of-Institutional-and- } \\
\text { Theoretical-Economics; } \\
\text { 155(1), March 1999, } \\
\text { pp. 176-80. }\end{array}$ & 1999 & Laux & Christian & U Mannheim & 0.29 & 0.5 & 1 & 0.145 & C. Laux: 0,145 \\
\hline $\begin{array}{l}\text { Multidimensional Mechanism Design } \\
\text { for Auctions with Externalities }\end{array}$ & $\begin{array}{l}\text { Jehiel,-P.; } \\
\text { Moldovanu,-B.; } \\
\text { Stacchetti,-E. }\end{array}$ & $\begin{array}{l}\text { Journal-of-Economic- } \\
\text { Theory; } 85(2) \text {, April } 1999, \\
\text { pp. 258-93. }\end{array}$ & 1999 & Moldovanu & Benny & U Mannheim & 0.93 & 1 & 3 & 0.465 & \\
\hline Goethe's Second-Price Auction & $\begin{array}{l}\text { Moldovanu,-- } \\
\text { Benny; Tietzel,- } \\
\text { Manfred } \\
\end{array}$ & $\begin{array}{l}\text { Journal-of-Political- } \\
\text { Economy; 106(4), August } \\
\text { 1998, pp. 854-59. }\end{array}$ & 1998 & Moldovanu & Benny & U Mannheim & 1.95 & 0.5 & 2 & 0.65 & \\
\hline How (Not) to Sell Nuclear Weapons & $\begin{array}{l}\text { Jehiel,-P.; } \\
\text { Moldovanu,-B.; } \\
\text { Stacchetti,-E. } \\
\end{array}$ & $\begin{array}{l}\text { American-Economic- } \\
\text { Review; 86(4), September } \\
\text { 1996, pp. 814-29. }\end{array}$ & 1996 & Moldovanu & Benny & U Mannheim & 1.74 & 1 & 3 & 0.87 & \\
\hline $\begin{array}{l}\text { The Production and Cost-Sharing of } \\
\text { an Excludable Public Good }\end{array}$ & $\begin{array}{l}\text { Moldovanu,-- } \\
\text { Benny } \\
\end{array}$ & $\begin{array}{l}\text { Economic-Theory; 7(3), } \\
\text { April 1996, pp. 531-39. }\end{array}$ & 1996 & Moldovanu & Benny & U Mannheim & 0 & 0.5 & 1 & 0 & \\
\hline $\begin{array}{l}\text { Cyclical Delay in Bargaining with } \\
\text { Externalities }\end{array}$ & $\begin{array}{l}\text { Jehiel,-Philippe; } \\
\text { Moldovanu,-- } \\
\text { Benny }\end{array}$ & $\begin{array}{l}\text { Review -of-Economic- } \\
\text { Studies; 62(4), October } \\
\text { 1995, pp. 619-37. }\end{array}$ & 1995 & Moldovanu & Benny & U Mannheim & 1.52 & 1 & 2 & 1.013333333 & \\
\hline Strategic Nonparticipation & $\begin{array}{l}\text { Jehiel,-Philippe; } \\
\text { Moldovanu,- } \\
\text { Benny }\end{array}$ & $\begin{array}{l}\text { Rand-Journal-of- } \\
\text { Economics; } 27(1) \text {, Spring } \\
\text { 1996, pp. 84-98. }\end{array}$ & 1996 & Moldovanu & Benny & U Mannheim & 1.12 & 1 & 2 & 0.746666667 & \\
\hline $\begin{array}{l}\text { Core Implementation and Increasing } \\
\text { Returns to Scale for Cooperation }\end{array}$ & $\begin{array}{l}\text { Moldovanu,-- } \\
\text { Benny; Winter,- } \\
\text { Eyal }\end{array}$ & $\begin{array}{l}\text { Journal-of-Mathematical- } \\
\text { Economics; } 23(6), \\
\text { November 1994, pp. 533- } \\
48 .\end{array}$ & 1994 & Moldovanu & Benny & U Mannheim & 0.37 & 1 & 2 & 0.246666667 & \\
\hline $\begin{array}{l}\text { Negative Externalities May Cause } \\
\text { Delay in Negotiation }\end{array}$ & $\begin{array}{l}\text { Jehiel,-Philippe; } \\
\text { Moldovanu,- } \\
\text { Benny }\end{array}$ & $\begin{array}{l}\text { Econometrica; 63(6), } \\
\text { November 1995, pp. 1321- } \\
35 . \\
\end{array}$ & 1995 & Moldovanu & Benny & U Mannheim & 2.22 & 1 & 2 & 1.48 & \\
\hline Order Independent Equilibria & $\begin{array}{l}\text { Moldovanu,-- } \\
\text { Benny; Winter,- } \\
\text { Eyal } \\
\end{array}$ & $\begin{array}{l}\text { Games-and-Economic- } \\
\text { Behavior; 9(1), April 1995, } \\
\text { pp. 21-34. }\end{array}$ & 1995 & Moldovanu & Benny & U Mannheim & 0.6 & 1 & 2 & 0.4 & \\
\hline $\begin{array}{l}\text { Price Indeterminacy and Bargaining in } \\
\text { a Market with Indivisibilities }\end{array}$ & $\begin{array}{l}\text { Moldovanu,-- } \\
\text { Benny }\end{array}$ & $\begin{array}{l}\text { Journal-of-Mathematical- } \\
\text { Economics; 22(6), 1993, } \\
\text { pp. 581-97. }\end{array}$ & 1993 & Moldovanu & Benny & U Mannheim & 0.37 & 1 & 1 & 0.37 & \\
\hline $\begin{array}{l}\text { Coalition-Proof Nash Equilibria and the } \\
\text { Core in Three-Player Games }\end{array}$ & $\begin{array}{l}\text { Moldovanu,-- } \\
\text { Benny }\end{array}$ & $\begin{array}{l}\text { Games-and-Economic- } \\
\text { Behavior; 4(4), October } \\
\text { 1992, pp. 565-81. }\end{array}$ & 1992 & Moldovanu & Benny & U Mannheim & 0.6 & 1 & 1 & 0.6 & $\begin{array}{l}\text { B. Moldovanu: } \\
6.841666667\end{array}$ \\
\hline $\begin{array}{l}\text { QALYs versus HYEs --What's Right } \\
\text { and What's Wrong: A Review of the } \\
\text { Controversy }\end{array}$ & Ried,-Walter & $\begin{array}{l}\text { Journal-of-Health- } \\
\text { Economics; } 17(5) \text {, October } \\
1998, \text { pp. } 607-25 .\end{array}$ & 1998 & Ried & Walter & U Mannheim & 1.39 & 1 & 1 & 1.39 & \\
\hline Comparative Dynamic Analysis of the & Ried,-Walter & Journal-of-Health- & 1998 & Ried & Walter & U Mannheim & 1.39 & 1 & 1 & 1.39 & \\
\hline
\end{tabular}


Universität Mannheim

\begin{tabular}{|c|c|c|c|c|c|c|c|c|c|c|c|}
\hline & Author(s) & Journal & Year & & & & $\begin{array}{l}\text { Impact } \\
\text { Factor }\end{array}$ & $\begin{array}{l}\text { Page- } \\
\text { corr. }\end{array}$ & Authors & Impact & personal Impact \\
\hline Full Grossman Model & & $\begin{array}{l}\text { Economics; 17(4), August } \\
\text { 1998, pp. 383-425. }\end{array}$ & & & & & & & & & \\
\hline $\begin{array}{l}\text { Willingness to Pay and Cost of Illness } \\
\text { for Changes in Health Capital } \\
\text { Depreciation }\end{array}$ & Ried,-Walter & $\begin{array}{l}\text { Health-Economics; } 5(5) \\
\text { Sept.-Oct. 1996, pp. 447- } \\
67 .\end{array}$ & 1996 & Ried & Walter & U Mannheim & 2.03 & 1 & 1 & 2.03 & W. Ried: 4.81 \\
\hline $\begin{array}{l}\text { The Employment Consequences of the } \\
\text { Sherman and Clayton Acts: Comment }\end{array}$ & Schlieper,-Ulrich & $\begin{array}{l}\text { Journal-of-Institutional-and- } \\
\text { Theoretical-Economics; } \\
\text { 147(1), March 1991, } \\
\text { pp. 58-59. }\end{array}$ & 1991 & Schlieper & Ulrich & U Mannheim & 0.29 & 0.5 & 1 & 0.145 & \\
\hline $\begin{array}{l}\text { Monitoring Agents with Other Agents: } \\
\text { Comment }\end{array}$ & Schlieper,-Ulrich & $\begin{array}{l}\text { Journal-of-Institutional-and- } \\
\text { Theoretical-Economics; } \\
\text { 146(1), March 1990, } \\
\text { pp. 175-76. }\end{array}$ & 1990 & Schlieper & Ulrich & U Mannheim & 0.29 & 0.5 & 1 & 0.145 & U. Schlieper: 0.29 \\
\hline $\begin{array}{l}\text { International Risk and Exchange Rate } \\
\text { Overshooting }\end{array}$ & $\begin{array}{l}\text { Schroeder,- } \\
\text { Juergen }\end{array}$ & $\begin{array}{l}\text { Journal-of-International- } \\
\text { Money-and-Finance; } 9(2) \\
\text { June 1990, pp. 193-205. }\end{array}$ & 1990 & Schröder & Jürgen & U Mannheim & 0.49 & 1 & 1 & 0.49 & J. Schröder: 0,49 \\
\hline Lone Mothers: A Review & $\begin{array}{l}\text { Staat,-Matthias; } \\
\text { Wagenhals,- } \\
\text { Gerhard }\end{array}$ & $\begin{array}{l}\text { Journal-of-Population- } \\
\text { Economics; } 9(2), \text { May } \\
\text { 1996, pp. 131-40. }\end{array}$ & 1996 & Staat & Matthias & U Mannheim & 0.44 & 0.5 & 2 & 0.146666667 & M. Staat: 0,14666 \\
\hline $\begin{array}{l}\text { Legal Form, Growth and Exit of West } \\
\text { German Firms --Empirical Results for } \\
\text { Manufacturing, Construction, Trade } \\
\text { and Service Industries }\end{array}$ & $\begin{array}{l}\text { Harhoff,-D.; } \\
\text { Stahl,-K.; } \\
\text { Woywode,-M. }\end{array}$ & $\begin{array}{l}\text { Journal-of-Industrial- } \\
\text { Economics; 46(4), } \\
\text { December 1998, pp. 453- } \\
88 .\end{array}$ & 1998 & Stahl & Konrad & U Mannheim & 0.6 & 1 & 3 & 0.3 & \\
\hline $\begin{array}{l}\text { On Income Tax Avoidance: The Case } \\
\text { of Germany }\end{array}$ & $\begin{array}{l}\text { Lang,-O.; } \\
\text { Nohrbass,-K.-H.; } \\
\text { Stahl,-K. }\end{array}$ & $\begin{array}{l}\text { Journal-of-Public- } \\
\text { Economics; 66(2), } \\
\text { November 1997, pp. 327- } \\
47 . \\
\end{array}$ & 1997 & Stahl & Konrad & U Mannheim & 0.82 & 1 & 3 & 0.41 & \\
\hline $\begin{array}{l}\text { Do Consumers Search for the Highest } \\
\text { Price? Oligopoly Equilibrium and } \\
\text { Monopoly Optimum in Differentiated- } \\
\text { Products Markets }\end{array}$ & $\begin{array}{l}\text { Schulz,-Norbert; } \\
\text { Stahl,-Konrad }\end{array}$ & $\begin{array}{l}\text { Rand-Journal-of- } \\
\text { Economics; 27(3), Autumn } \\
\text { 1996, pp. 542-62. }\end{array}$ & 1996 & Stahl & Konrad & U Mannheim & 1.13 & 1 & 2 & 0.753333333 & \\
\hline $\begin{array}{l}\text { Unternehmens- und } \\
\text { Beschaftigungsdynamik in } \\
\text { Westdeutschland: Zum Einfluss von } \\
\text { Haftungsregein und } \\
\text { Eigentumerstruktur. }\end{array}$ & $\begin{array}{l}\text { Harhoff,-Dietmar; } \\
\text { Stahl,-Konrad }\end{array}$ & $\begin{array}{l}\text { Ifo-Studien; 41(1), 1995, } \\
\text { pp. 17-50. }\end{array}$ & 1995 & Stahl & Konrad & U Mannheim & 0 & 1 & 2 & 0 & \\
\hline $\begin{array}{l}\text { Corrigendum: Good and Bad } \\
\text { Competition in Spatial Markets for } \\
\text { Search Goods: The Case of Linear } \\
\text { Utility Functions }\end{array}$ & $\begin{array}{l}\text { Schulz,-Norbert; } \\
\text { Stahl,-Konrad }\end{array}$ & $\begin{array}{l}\text { Annales-d'Economie-et-de- } \\
\text { Statistique; 0(28), Oct.- } \\
\text { Dec. 1992, pp. 165-66. }\end{array}$ & 1992 & Stahl & Konrad & U Mannheim & 0 & 0.5 & 2 & 0 & \\
\hline $\begin{array}{l}\text { Life Cycle Savings and Consumption } \\
\text { Constraints: Theory, Empirical } \\
\text { Evidence, and Fiscal Implications }\end{array}$ & $\begin{array}{l}\text { Borsch-Supan,-- } \\
\text { Axel-H.; Stahl,-- } \\
\text { Konrad }\end{array}$ & $\begin{array}{l}\text { Journal-of-Population- } \\
\text { Economics; 4(3), August } \\
\text { 1991, pp. 233-55. }\end{array}$ & 1991 & Stahl & Konrad & U Mannheim & 0.44 & 1 & 2 & 0.293333333 & \\
\hline $\begin{array}{l}\text { Do Savings Programs Dedicated to } \\
\text { Home-Ownership Increase Personal } \\
\text { Savings? }\end{array}$ & $\begin{array}{l}\text { Borsch-Supan,-- } \\
\text { Axel; Stahl,-- } \\
\text { Konrad }\end{array}$ & $\begin{array}{l}\text { Journal-of-Public- } \\
\text { Economics; 44(3), April } \\
\text { 1991, pp. 265-97. }\end{array}$ & 1991 & Stahl & Konrad & U Mannheim & 0.82 & 1 & 2 & 0.546666667 & K. Stahl: \\
\hline $\begin{array}{l}\text { Recent Issues in the Economics of } \\
\text { Housing Markets: Introduction }\end{array}$ & Stahl,-Konrad & $\begin{array}{l}\text { Regional-Science-and- } \\
\text { Urban-Economics; } 20(1),\end{array}$ & 1990 & Stahl & Konrad & U Mannheim & 0.56 & 0.5 & 1 & 0.28 & 2.583333333 \\
\hline
\end{tabular}


Universität Mannheim

\begin{tabular}{|c|c|c|c|c|c|c|c|c|c|c|c|}
\hline & Author(s) & Journal & Year & & & & \begin{tabular}{l|} 
Impact \\
Factor
\end{tabular} & $\begin{array}{l}\text { Page- } \\
\text { corr. }\end{array}$ & Authors & Impact & personal Impact \\
\hline & & June 1990, pp. 1-4. & & & & & & & & & \\
\hline $\begin{array}{l}\text { Enforcing Competition among } \\
\text { Governments: Theory and Application } \\
\text { to the European Union }\end{array}$ & Vaubel,-Roland & $\begin{array}{l}\text { Constitutional-Political- } \\
\text { Economy; 10(4), November } \\
\text { 1999, pp. 327-38. }\end{array}$ & 1999 & Vaubel & Roland & U Mannheim & 0 & 1 & 1 & 0 & \\
\hline $\begin{array}{l}\text { Reply to Berger and Woitek [How } \\
\text { Opportunistic Are Partisan German } \\
\text { Central Bankers: Evidence on the } \\
\text { Vaubel Hyppothesis]. }\end{array}$ & Vaubel,-Roland & $\begin{array}{l}\text { European-Journal-of- } \\
\text { Political-Economy; 13(4), } \\
\text { December 1997, pp. 823- } \\
27 .\end{array}$ & 1997 & Vaubel & Roland & U Mannheim & 0 & 0.5 & 1 & 0 & \\
\hline $\begin{array}{l}\text { How Opportunistic Are Partisan } \\
\text { German Central Bankers: Evidence } \\
\text { on the Vaubel Hypothesis }\end{array}$ & $\begin{array}{l}\text { Berger,-Helge; } \\
\text { Woitek,-Ulrich }\end{array}$ & $\begin{array}{l}\text { European-Journal-of- } \\
\text { Political-Economy; 13(4), } \\
\text { December 1997, pp. 807- } \\
21 .\end{array}$ & 1997 & Vaubel & Roland & U Mannheim & 0 & 0.5 & 2 & 0 & \\
\hline $\begin{array}{l}\text { The Bureaucratic and Partisan } \\
\text { Behavior of Independent Central } \\
\text { Banks: German and International } \\
\text { Evidence }\end{array}$ & Vaubel,-Roland & $\begin{array}{l}\text { European-Journal-of- } \\
\text { Political-Economy; } 13(2), \\
\text { May 1997, pp. 201-24. }\end{array}$ & 1997 & Vaubel & Roland & U Mannheim & 0 & 1 & 1 & 0 & \\
\hline $\begin{array}{l}\text { The Constitutional Reform of the } \\
\text { European Union }\end{array}$ & Vaubel,-Roland & $\begin{array}{l}\text { European-Economic- } \\
\text { Review; } 41(3-5) \text {, April } \\
\text { 1997, pp. 443-50. }\end{array}$ & 1997 & Vaubel & Roland & U Mannheim & 0.84 & 0.5 & 1 & 0.42 & \\
\hline $\begin{array}{l}\text { The Constitutional Future of the } \\
\text { European Union }\end{array}$ & Vaubel,-Roland & $\begin{array}{l}\text { Constitutional-Political- } \\
\text { Economy; } 7(4), 1996 \text {, } \\
\text { pp. 317-24. }\end{array}$ & 1996 & Vaubel & Roland & U Mannheim & 0 & 0.5 & 1 & 0 & \\
\hline $\begin{array}{l}\text { Constitutional Safeguards against } \\
\text { Centralization in Federal States: An } \\
\text { International Cross-Section Analysis }\end{array}$ & Vaubel,-Roland & $\begin{array}{l}\text { Constitutional-Political- } \\
\text { Economy; } 7(2), 1996 \text {, } \\
\text { pp. } 79-102 .\end{array}$ & 1996 & Vaubel & Roland & U Mannheim & 0 & 1 & 1 & 0 & \\
\hline $\begin{array}{l}\text { Bureaucracy at the IMF and the World } \\
\text { Bank: A Comparison of the Evidence }\end{array}$ & Vaubel,-Roland & $\begin{array}{l}\text { World-Economy; 19(2), } \\
\text { March 1996, pp. 195-210. }\end{array}$ & 1996 & Vaubel & Roland & U Mannheim & 0.49 & 1 & 1 & 0.49 & \\
\hline $\begin{array}{l}\text { Social Regulation and Market } \\
\text { Integration: A Critique and Public- } \\
\text { Choice Analysis of the Social Chapter }\end{array}$ & Vaubel,-Roland & $\begin{array}{l}\text { Aussenwirtschaft; 50(1), } \\
\text { April 1995, pp. 111-31. }\end{array}$ & 1995 & Vaubel & Roland & U Mannheim & 0 & 1 & 1 & 0 & \\
\hline $\begin{array}{l}\text { The Political Economy of } \\
\text { Centralization and the European } \\
\text { Community }\end{array}$ & Vaubel,-Roland & $\begin{array}{l}\text { Public-Choice; 81(1-2), } \\
\text { October 1994, pp. 151-90. }\end{array}$ & 1994 & Vaubel & Roland & U Mannheim & 0.43 & 1 & 1 & 0.43 & \\
\hline $\begin{array}{l}\text { The Public Choice Analysis of } \\
\text { European Integration: A Survey }\end{array}$ & Vaubel,-Roland & $\begin{array}{l}\text { European-Journal-of- } \\
\text { Political-Economy; 10(1), } \\
\text { May 1994, pp. 227-49. }\end{array}$ & 1994 & Vaubel & Roland & U Mannheim & 0 & 1 & 1 & 0 & \\
\hline $\begin{array}{l}\text { The Delors Committee and Report: A } \\
\text { Public Choice Analysis }\end{array}$ & Vaubel,-Roland & $\begin{array}{l}\text { Cato-Journal; } 10(2) \text {, Fall } \\
1990, \text { pp. } 419-21 .\end{array}$ & 1990 & Vaubel & Roland & U Mannheim & 0 & 1 & 1 & 0 & \\
\hline $\begin{array}{l}\text { Currency Competition and European } \\
\text { Monetary Integration }\end{array}$ & Vaubel,-Roland & $\begin{array}{l}\text { Economic-Journal; } \\
\text { 100(402), September 1990, } \\
\text { pp. 936-46. }\end{array}$ & 1990 & Vaubel & Roland & U Mannheim & 1.21 & 1 & 1 & 1.21 & R. Vaubel 2.55 \\
\hline $\begin{array}{l}\text { Pay-as-You-Go Financed Public } \\
\text { Pensions in a Model of Endogenous } \\
\text { Growth and Fertility }\end{array}$ & $\begin{array}{l}\text { Wigger,-Berthold- } \\
\text { U. }\end{array}$ & $\begin{array}{l}\text { Journal-of-Population- } \\
\text { Economics; } 12(4), \\
\text { November 1999, pp. 625- } \\
40 .\end{array}$ & 1999 & Wigger & Berthold U. & U Mannheim & 0.44 & 1 & 1 & 0.44 & \\
\hline $\begin{array}{l}\text { Two-Sided Altruism, the Samaritan's } \\
\text { Dilemma, and Universal Compulsory }\end{array}$ & $\begin{array}{l}\text { Wigger,-Berthold- } \\
\text { U. }\end{array}$ & $\begin{array}{l}\text { Public-Finance; } 51(2), \\
1996, \text { pp. } 275-90 .\end{array}$ & 1996 & Wigger & Berthold U. & U Mannheim & 0 & 1 & 1 & 0 & \\
\hline
\end{tabular}


Universität Mannheim

\begin{tabular}{|c|c|c|c|c|c|c|c|c|c|c|c|}
\hline & Author(s) & Journal & Year & & & & $\begin{array}{l}\text { Impact } \\
\text { Factor }\end{array}$ & $\begin{array}{l}\text { Page- } \\
\text { corr. }\end{array}$ & Authors & Impact & personal Impact \\
\hline \multicolumn{12}{|l|}{ Insurance } \\
\hline $\begin{array}{l}\text { Consumption and Wage Taxes When } \\
\text { Household Size Matters }\end{array}$ & $\begin{array}{l}\text { Wigger,-Berthold- } \\
\text { U. }\end{array}$ & $\begin{array}{l}\text { Economics-Letters; 54(2), } \\
\text { February 1997, pp. 139-42. }\end{array}$ & 1997 & Wigger & Berthold U. & U Mannheim & 0.26 & 0.5 & 1 & 0.13 & 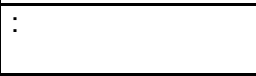 \\
\hline $\begin{array}{l}\text { Convergence and Divergence of } \\
\text { Economic Growth in a Two-Country } \\
\text { Model without Externalities }\end{array}$ & $\begin{array}{l}\text { Wigger,-Berthold- } \\
\text { U. }\end{array}$ & $\begin{array}{l}\text { Journal-of-Economic- } \\
\text { Integration; 11(2), June } \\
\text { 1996, pp. 160-78. }\end{array}$ & 1996 & Wigger & Berthold U. & U Mannheim & 0 & 1 & 1 & 0 & B. U. Wigger 0.57 \\
\hline $\begin{array}{l}\text { Healthcare Reform and Expenditure } \\
\text { on Drugs: The German Situation }\end{array}$ & $\begin{array}{l}\text { Ulrich,-Volker; } \\
\text { Wille,-Eberhard }\end{array}$ & $\begin{array}{l}\text { PharmacoEconomics; } \\
\text { 10(0), Suppl. } 2 \text { 1996, } \\
\text { pp. 81-88. } \\
\end{array}$ & 1996 & Wille & Eberhard & U Mannheim & 0 & 0.5 & 2 & 0 & E. Wille: 0 \\
\hline $\begin{array}{l}\text { Ein modifiziertes verteilungsfreies } \\
\text { Kleinste-Quadrate-Pendant zu } \\
\text { Johansens Maximum-Likelihood- } \\
\text { Schatzung der Parameter in ... }\end{array}$ & Winckler,-Klaus & $\begin{array}{l}\text { Ifo-Studien; 41(4), 1995, } \\
\text { pp. 561-76. }\end{array}$ & 1995 & Winckler & Klaus & U Mannheim & 0 & 1 & 1 & 0 & K. Winckler: 0 \\
\hline $\begin{array}{l}\text { Causes and Effects of Financing } \\
\text { Constraints at the Firm Level }\end{array}$ & Winker,-Peter & $\begin{array}{l}\text { Small-Business- } \\
\text { Economics; 12(2), March } \\
\text { 1999, pp. 169-81. }\end{array}$ & 1999 & Winker & Peter & U Mannheim & 0.35 & 1 & 1 & 0.35 & \\
\hline $\begin{array}{l}\text { Zufall und Quasi-Monte Carlo Ansatze: } \\
\text { Einige Anmerkungen zu Grundlagen } \\
\text { und Anwendungen in Statistik und } \\
\text { Okonometrie. }\end{array}$ & $\begin{array}{l}\text { Winker,-Peter; } \\
\text { Fang,-Kai-Tai }\end{array}$ & $\begin{array}{l}\text { Jahrbucher-fur- } \\
\text { Nationalokonomie-und- } \\
\text { Statistik; 218(1-2), January } \\
\text { 1999, pp. 215-28. } \\
\end{array}$ & 1999 & Winker & Peter & U Mannheim & 0.21 & 1 & 2 & 0.14 & \\
\hline $\begin{array}{l}\text { Measuring the Fiscal Revenue Loss of } \\
\text { VAT Exemption in Commercial } \\
\text { Banking }\end{array}$ & $\begin{array}{l}\text { Genser,-Bernd; } \\
\text { Winker,-Peter }\end{array}$ & $\begin{array}{l}\text { FinanzArchiv; 54(4), 1997, } \\
\text { pp. 563-85. }\end{array}$ & 1997 & Winker & Peter & U Mannheim & 0 & 1 & 2 & 0 & \\
\hline $\begin{array}{l}\text { Die Tragheit von Zinssatzen und } \\
\text { Kreditrationierung in der } \\
\text { Bundesrepublik Deutschland. }\end{array}$ & Winker,-Peter & $\begin{array}{l}\text { Jahrbucher-fur- } \\
\text { Nationalokonomie-und- } \\
\text { Statistik; 214(2), March } \\
\text { 1995, pp. 154-74. }\end{array}$ & 1995 & Winker & Peter & U Mannheim & 0.21 & 1 & 1 & 0.21 & \\
\hline $\begin{array}{l}\text { A Macroeconometric Disequilibrium } \\
\text { Analysis of Current and Future } \\
\text { Migration from Eastern Europe into } \\
\text { West Germany }\end{array}$ & $\begin{array}{l}\text { Franz,-W.; Oser,-- } \\
\text { U.; Winker,-P. }\end{array}$ & $\begin{array}{l}\text { Journal-of-Population- } \\
\text { Economics; 7(2), 1994, } \\
\text { pp. 217-34. }\end{array}$ & 1994 & Winker & Peter & U Mannheim & 0.44 & 1 & 3 & 0.22 & \\
\hline $\begin{array}{l}\text { Firmenalter un Kreditrationierung: } \\
\text { Eine mikrookonometrische Analyse mit } \\
\text { ifo Umfragedaten. }\end{array}$ & Winker,-Peter & $\begin{array}{l}\text { Ifo-Studien; 39(2), 1993, } \\
\text { pp. 103-26. }\end{array}$ & 1993 & Winker & Peter & U Mannheim & 0 & 1 & 1 & 0 & P. Winker:0.92 \\
\hline
\end{tabular}

Average: 0.886589744 


\section{Universität Salzburg}

\begin{tabular}{|c|c|c|c|c|c|c|c|c|c|c|c|}
\hline & Author & Journal & Year & & & & $\begin{array}{l}\text { Impact } \\
\text { Faktor }\end{array}$ & $\begin{array}{l}\text { Seiten- } \\
\text { korr. }\end{array}$ & Autoren & Impact & personal Impact \\
\hline $\begin{array}{l}\text { The Development of Professor Hayek's } \\
\text { Research Programme }\end{array}$ & Tschiderer,-Werner & \begin{tabular}{|l|} 
International-Journal-of- \\
Social-Economics; 22(6), \\
1995, pp. 65-80.
\end{tabular} & 1995 & Tschiderer & Werner & U Salzburg & 0 & 1 & 1 & 0 & \\
\hline $\begin{array}{l}\text { The Development of Professor Hayek's } \\
\text { Research Programme: Erratum }\end{array}$ & Tschiderer,-Werner & $\begin{array}{l}\text { International-Journal-of- } \\
\text { Social-Economics; 22(5), } \\
\text { 1995, pp. } 80 .\end{array}$ & 1995 & Tschiderer & Werner & U Salzburg & 0 & 0.5 & 1 & 0 & \\
\hline $\begin{array}{l}\text { The Development of Professor Hayek's } \\
\text { Research Programme }\end{array}$ & Tschiderer,-Werner & $\begin{array}{l}\text { International-Journal-of- } \\
\text { Social-Economics; 22(2), } \\
\text { 1995, pp. 61-76. }\end{array}$ & 1995 & Tschiderer & Werner & U Salzburg & 0 & 1 & 1 & 0 & W. Tschiderer: 0 \\
\hline
\end{tabular}


Universität Wien

\begin{tabular}{|c|c|c|c|c|c|c|c|c|c|c|c|}
\hline & Author(s) & Journal & Year & & & & $\begin{array}{l}\text { Impact } \\
\text { Factor }\end{array}$ & $\begin{array}{l}\text { Page- } \\
\text { corr. }\end{array}$ & Authors & Impact & personal Impact \\
\hline Adverse Selection and Pigou Taxes & Clemenz,-Gerhard & $\begin{array}{l}\text { Environmental-and- } \\
\text { Resource-Economics; } \\
\text { 13(1), January 1999, } \\
\text { pp. 13-29. }\end{array}$ & 1999 & Clemenz & Gerhard & Uni-Wien & 0 & 1 & 1 & 0 & \\
\hline $\begin{array}{l}\text { Credit Markets with Moral Hazard and } \\
\text { Heterogeneous Valuations of Collateral }\end{array}$ & $\begin{array}{l}\text { Bell,-Clive; } \\
\text { Clemenz,-Gerhard }\end{array}$ & $\begin{array}{l}\text { Research-in-Economics; } \\
\text { 52(3), September 1998, } \\
\text { pp. 285-309. }\end{array}$ & 1998 & Clemenz & Gerhard & Uni-Wien & 0 & 1 & 2 & 0 & \\
\hline $\begin{array}{l}\text { Adverse Selection in Labor Markets } \\
\text { and International Trade }\end{array}$ & Clemenz,-Gerhard & $\begin{array}{l}\text { Scandinavian-Journal-of- } \\
\text { Economics; 97(1), March } \\
1995, \text { pp. } 73-88 .\end{array}$ & 1995 & Clemenz & Gerhard & Uni-Wien & 0.48 & 1 & 1 & 0.48 & \\
\hline $\begin{array}{l}\text { Competition via Shopping Hours: A } \\
\text { Case for Regulation? }\end{array}$ & Clemenz,-Gerhard & $\begin{array}{l}\text { Journal-of-Institutional- } \\
\text { and-Theoretical- } \\
\text { Economics; } 150(4) \text {, } \\
\text { December 1994, pp. 625- } \\
41 . \\
\end{array}$ & 1994 & Clemenz & Gerhard & Uni-Wien & 0.29 & 1 & 1 & 0.29 & \\
\hline $\begin{array}{l}\text { Forschung und Entwicklung als } \\
\text { Suchprozess bei oligopolistischem } \\
\text { Wettbewerb. }\end{array}$ & Clemenz,-Gerhard & $\begin{array}{l}\text { Ifo-Studien; 39(3-4), 1993, } \\
\text { pp. 257-77. }\end{array}$ & 1993 & Clemenz & Gerhard & Uni-Wien & 0 & 1 & 1 & 0 & \\
\hline $\begin{array}{l}\text { A Note on the Nonexistence of a } \\
\text { Rationing Equilibrium in the Besanko- } \\
\text { Thakor Model }\end{array}$ & Clemenz,-Gerhard & $\begin{array}{l}\text { International-Economic- } \\
\text { Review; 34(3), August } \\
\text { 1993, pp. 727-37. }\end{array}$ & 1993 & Clemenz & Gerhard & Uni-Wien & 0.69 & 1 & 1 & 0.69 & \\
\hline $\begin{array}{l}\text { Credit Markets with Asymmetric } \\
\text { Information: A Survey }\end{array}$ & $\begin{array}{l}\text { Clemenz,-Gerhard; } \\
\text { Ritthaler,-Mona }\end{array}$ & $\begin{array}{l}\text { Finnish-Economic-Papers; } \\
\text { 5(1), Spring 1992, pp. 12- } \\
26 .\end{array}$ & 1992 & Clemenz & Gerhard & Uni-Wien & 0 & 1 & 2 & 0 & \\
\hline Market Structure and R\&D Competition & Clemenz,-Gerhard & $\begin{array}{l}\text { European-Economic- } \\
\text { Review; 36(4), May 1992, } \\
\text { pp. 847-64. }\end{array}$ & 1992 & Clemenz & Gerhard & Uni-Wien & 0.84 & 1 & 1 & 0.84 & \\
\hline $\begin{array}{l}\text { International Impact of Austrian } \\
\text { Economists }\end{array}$ & $\begin{array}{l}\text { Clemenz,-Gerhard; } \\
\text { Neusser,-Klaus }\end{array}$ & $\begin{array}{l}\text { Empirica; 18(2), 1991, } \\
\text { pp. 201-19. }\end{array}$ & 1991 & Clemenz & Gerhard & Uni-Wien & 0 & 1 & 2 & 0 & \\
\hline $\begin{array}{l}\text { Loans for Projects with Interdependent } \\
\text { Returns }\end{array}$ & Clemenz,-Gerhard & $\begin{array}{l}\text { Journal-of-Institutional- } \\
\text { and-Theoretical- } \\
\text { Economics; } 147(2) \text {, June } \\
\text { 1991, pp. 337-53. }\end{array}$ & 1991 & Clemenz & Gerhard & Uni-Wien & 0.29 & 1 & 1 & 0.29 & \\
\hline Optimal Price-Cap Regulation & Clemenz,-Gerhard & $\begin{array}{l}\text { Journal-of-Industrial- } \\
\text { Economics; 39(4), June } \\
\text { 1991, pp. 391-408. }\end{array}$ & 1991 & Clemenz & Gerhard & Uni-Wien & 0.6 & 1 & 1 & 0.6 & \\
\hline $\begin{array}{l}\text { Non-sequential Consumer Search and } \\
\text { the Consequences of a Deregulation of } \\
\text { Trading Hours }\end{array}$ & \begin{tabular}{|l} 
Clemenz,-Gerhard \\
\end{tabular} & $\begin{array}{l}\text { European-Economic- } \\
\text { Review; 34(7), November } \\
\text { 1990, pp. 1323-37. }\end{array}$ & 1990 & Clemenz & Gerhard & Uni-Wien & 0.84 & 1 & 1 & 0.84 & \\
\hline $\begin{array}{l}\text { International R\&D Competition and } \\
\text { Trade Policy }\end{array}$ & Clemenz,-Gerhard & $\begin{array}{l}\text { Journal-of-International- } \\
\text { Economics; 28(1/2), } \\
\text { February 1990, pp. 93- } \\
113 .\end{array}$ & 1990 & Clemenz & Gerhard & Uni-Wien & 0.8 & 1 & 1 & 0.8 & G. Clemenz: 4.83 \\
\hline $\begin{array}{l}\text { The Price Normalization Problem in } \\
\text { Imperfect Competition and the } \\
\text { Objective of the Firm }\end{array}$ & $\begin{array}{l}\text { Dierker,-Egbert; } \\
\text { Grodal,-Birgit }\end{array}$ & $\begin{array}{l}\text { Economic-Theory; 14(2), } \\
\text { August 1999, pp. 257-84. }\end{array}$ & 1999 & Dierker & Egbert & Uni-Wien & 0 & 1 & 2 & 0 & \\
\hline
\end{tabular}


Universität Wien

\begin{tabular}{|c|c|c|c|c|c|c|c|c|c|c|c|}
\hline & Author(s) & Journal & Year & & & & $\begin{array}{l}\text { Impact } \\
\text { Factor }\end{array}$ & $\begin{array}{l}\text { Page- } \\
\text { corr. }\end{array}$ & Authors & Impact & personal Impact \\
\hline $\begin{array}{l}\text { Modelling Policy Issues in a World of } \\
\text { Imperfect Competition }\end{array}$ & $\begin{array}{l}\text { Dierker,-Egbert; } \\
\text { Grodal,--Birgit }\end{array}$ & $\begin{array}{l}\text { Scandinavian-Journal-of- } \\
\text { Economics; 100(1), March } \\
\text { 1998, pp. 153-79. }\end{array}$ & 1998 & Dierker & Egbert & Uni-Wien & 0.48 & 1 & 2 & 0.32 & \\
\hline $\begin{array}{l}\text { Profit Maximization Mitigates } \\
\text { Competition }\end{array}$ & $\begin{array}{l}\text { Dierker,-Egbert; } \\
\text { Grodal,-Birgit }\end{array}$ & $\begin{array}{l}\text { Economic-Theory; } 7(1), \\
\text { January } 1996, \text { pp. } 139-60 .\end{array}$ & 1996 & Dierker & Egbert & Uni-Wien & 0 & 1 & 2 & 0 & \\
\hline $\begin{array}{l}\text { The Optimality of Boiteux-Ramsey } \\
\text { Pricing }\end{array}$ & Dierker,-Egbert & $\begin{array}{l}\text { Econometrica; } 59(1) \\
\text { January } 1991, \text { pp. } 99-121 .\end{array}$ & 1991 & Dierker & Egbert & Uni-Wien & 2.22 & 1 & 1 & 2.22 & \\
\hline $\begin{array}{l}\text { Tax Systems and Direct Mechanisms in } \\
\text { Large Finite Economies }\end{array}$ & $\begin{array}{l}\text { Dierker,-Egbert; } \\
\text { Haller,-Hans }\end{array}$ & $\begin{array}{l}\text { Journal-of-Economics- } \\
\text { (Zeitschrift-fur- } \\
\text { Nationalokonomie); 52(2), } \\
\text { 1990, pp. 99-116. }\end{array}$ & 1990 & Dierker & Egbert & Uni-Wien & 0.18 & 1 & 2 & 0.12 & E. Dieker:2.66 \\
\hline The Absent-Minded Centipede & $\begin{array}{l}\text { Dulleck,-Uwe; } \\
\text { Oechssler,-Jorg }\end{array}$ & $\begin{array}{l}\text { Economics-Letters; 55(3), } \\
\text { September 1997, pp. 309- } \\
15 .\end{array}$ & 1997 & Dulleck & Uwe & Uni-Wien & 0.26 & 0.5 & 2 & 0.086666667 & U. Dullek: 0.08667 \\
\hline $\begin{array}{l}\text { Corporate Ownership Structure in } \\
\text { Austria }\end{array}$ & Gugler,-Klaus & $\begin{array}{l}\text { Empirica; 25(3), 1998, } \\
\text { pp. 285-307. }\end{array}$ & 1998 & Gugler & Klaus & Uni-Wien & 0 & 1 & 2 & 0 & K. Gugler: 0 \\
\hline $\begin{array}{l}\text { Multiplicity of Cournot Equilibria and } \\
\text { Involuntary Unemployment }\end{array}$ & Kaas,-Leo & $\begin{array}{l}\text { Journal-of-Economic- } \\
\text { Theory; 80(2), June 1998, } \\
\text { pp. 332-49. }\end{array}$ & 1998 & Kaas & Leo & Uni-Wien & 0.93 & 1 & 1 & 0.93 & \\
\hline $\begin{array}{l}\text { Stabilizing Chaos in a Dynamic } \\
\text { Macroeconomic Model }\end{array}$ & Kaas,-Leo & $\begin{array}{l}\text { Journal-of-Economic- } \\
\text { Behavior-and- } \\
\text { Organization; 33(3-4), } \\
\text { Special Issue January } \\
\text { 1998, pp. 313-32. }\end{array}$ & 1998 & Kaas & Leo & Uni-Wien & 0.42 & 1 & 1 & 0.42 & L. Kaas: 1.35 \\
\hline $\begin{array}{l}\text { Permanent Components in Swiss } \\
\text { Macroeconomic Variables }\end{array}$ & Kaufmann,-Sylvia & $\begin{array}{l}\text { Schweizerische- } \\
\text { Zeitschrift-fur- } \\
\text { Volkswirtschaft- und } \\
\text { Statistik; 132(4/1), Dec. } \\
\text { 1996, pp. 539-61. }\end{array}$ & 1996 & Kaufmann & Sylvia & Uni-Wien & 0 & 1 & 1 & 0 & S. Kaufmann: 0 \\
\hline $\begin{array}{l}\text { Voluntary Contributions When the } \\
\text { Public Good Is Not Necessarily Normal }\end{array}$ & $\begin{array}{l}\text { Kerschbamer,-- } \\
\text { Rudolf; Puppe,- } \\
\text { Clemens }\end{array}$ & $\begin{array}{l}\text { Journal-of-Economics- } \\
\text { (Zeitschrift-fur- } \\
\text { Nationalokonomie); 68(2), } \\
\text { 1998, pp. 175-92. }\end{array}$ & 1998 & Kerschbamer & Rudolf & Uni-Wien & 0.18 & 1 & 2 & 0.12 & \\
\hline $\begin{array}{l}\text { Are Two a Good Representative for } \\
\text { Many? }\end{array}$ & $\begin{array}{l}\text { Kerschbamer,- } \\
\text { Rudolf; Maderner,- } \\
\text { Nina } \\
\end{array}$ & $\begin{array}{l}\text { Journal-of-Economic- } \\
\text { Theory; 83(1), November } \\
\text { 1998, pp. 90-104. }\end{array}$ & 1998 & Kerschbamer & Rudolf & Uni-Wien & 0.93 & 1 & 2 & 0.62 & \\
\hline $\begin{array}{l}\text { Disciplinary Takeovers and Industry } \\
\text { Effects }\end{array}$ & $\begin{array}{l}\text { Kerschbamer,- } \\
\text { Rudolf }\end{array}$ & $\begin{array}{l}\text { Journal-of-Economics- } \\
\text { and-Management- } \\
\text { Strategy; } 7(2) \text {, Summer } \\
\text { 1998, pp. 265-306. }\end{array}$ & 1998 & Kerschbamer & Rudolf & Uni-Wien & 0 & 1 & 1 & 0 & \\
\hline $\begin{array}{l}\text { Information Revelation via Takeovers in } \\
\text { Correlated Environments }\end{array}$ & $\begin{array}{l}\text { Kerschbamer,- } \\
\text { Rudolf }\end{array}$ & $\begin{array}{l}\text { Managerial-and-Decision- } \\
\text { Economics; } 18(1), \\
\text { February } 1997 \text {, pp. } 55-60 .\end{array}$ & 1997 & Kerschbamer & Rudolf & Uni-Wien & 0 & 0.5 & 1 & 0 & \\
\hline $\begin{array}{l}\text { Destroying the "Pretending" Equilibria } \\
\text { in the Demski-Sappington-Spiller Model }\end{array}$ & $\begin{array}{l}\text { Kerschbamer,- } \\
\text { Rudolf }\end{array}$ & $\begin{array}{l}\text { Journal-of-Economic- } \\
\text { Theory; } 62(1), \text { February } \\
\text { 1994, pp. } 230-37 .\end{array}$ & 1994 & Kerschbamer & Rudolf & Uni-Wien & 0.93 & 0.5 & 1 & 0.465 & $\begin{array}{l}\text { R. Kerschbamer: } \\
1.205\end{array}$ \\
\hline
\end{tabular}


Universität Wien

\begin{tabular}{|c|c|c|c|c|c|c|c|c|c|c|c|}
\hline & Author(s) & Journal & Year & & & & $\begin{array}{l}\text { Impact } \\
\text { Factor }\end{array}$ & $\begin{array}{l}\text { Page- } \\
\text { corr. }\end{array}$ & Authors & Impact & personal Impact \\
\hline $\begin{array}{l}\text { Imperfectly Observable Commitments } \\
\text { in n-Player Games }\end{array}$ & $\begin{array}{l}\text { Guth,-Werner; } \\
\text { Kirchsteiger,-Georg; } \\
\text { Ritzberger,-Klaus }\end{array}$ & $\begin{array}{l}\text { Games-and-Economic- } \\
\text { Behavior; } 23(1) \text {, April } \\
\text { 1998, pp. 54-74. }\end{array}$ & 1998 & Kirchsteiger & Georg & Uni-Wien & 0.6 & 1 & 3 & 0.3 & \\
\hline $\begin{array}{l}\text { Gift Exchange and Reciprocity in } \\
\text { Competitive Experimental Markets }\end{array}$ & $\begin{array}{l}\text { Fehr,-Ernst; } \\
\text { Kirchsteiger,-Georg; } \\
\text { Riedl,-Arno }\end{array}$ & $\begin{array}{l}\text { European-Economic- } \\
\text { Review; 42(1), January } \\
\text { 1998, pp. 1-34. } \\
\end{array}$ & 1998 & Kirchsteiger & Georg & Uni-Wien & 0.84 & 1 & 3 & 0.42 & \\
\hline $\begin{array}{l}\text { On the Possibility of Efficient Private } \\
\text { Provision of Public Goods through } \\
\text { Government Subsidies }\end{array}$ & $\begin{array}{l}\text { Kirchsteiger,-Georg; } \\
\text { Puppe,-Clemens }\end{array}$ & $\begin{array}{l}\text { Journal-of-Public- } \\
\text { Economics; 66(3), } \\
\text { December 1997, pp. 489- } \\
504 . \\
\end{array}$ & 1997 & Kirchsteiger & Georg & Uni-Wien & 0.82 & 1 & 2 & 0.546666667 & \\
\hline On the Formation of Political Coalitions & $\begin{array}{l}\text { Kirchsteiger,-Georg; } \\
\text { Puppe,-Clemens }\end{array}$ & $\begin{array}{l}\text { Journal-of-Institutional- } \\
\text { and-Theoretical- } \\
\text { Economics; 153(2), June } \\
\text { 1997, pp. 293-319. } \\
\end{array}$ & 1997 & Kirchsteiger & Georg & Uni-Wien & 0.29 & 1 & 2 & 0.193333333 & \\
\hline $\begin{array}{l}\text { Reciprocity as a Contract Enforcement } \\
\text { Device: Experimental Evidence }\end{array}$ & $\begin{array}{l}\text { Fehr,-Ernst; } \\
\text { Gachter,-Simon; } \\
\text { Kirchsteiger,-Georg } \\
\end{array}$ & $\begin{array}{l}\text { Econometrica; 65(4), July } \\
\text { 1997, pp. 833-60. }\end{array}$ & 1997 & Kirchsteiger & Georg & Uni-Wien & 2.22 & 1 & 3 & 1.11 & \\
\hline $\begin{array}{l}\text { Reciprocal Fairness and } \\
\text { Noncompensating Wage Differentials }\end{array}$ & \begin{tabular}{|l|} 
Fehr,-Ernst; \\
Gachter,-Simon; \\
Kirchsteiger,-Georg
\end{tabular} & $\begin{array}{l}\text { Journal-of-Institutional- } \\
\text { and-Theoretical- } \\
\text { Economics; 152(4), } \\
\text { December 1996, pp. 608- } \\
40 .\end{array}$ & 1996 & Kirchsteiger & Georg & Uni-Wien & 0.29 & 1 & 3 & 0.145 & \\
\hline $\begin{array}{l}\text { Intransitive Choices Based on } \\
\text { Transitive Preferences: The Case of } \\
\text { Menu-Dependent Information }\end{array}$ & $\begin{array}{l}\text { Kirchsteiger,-Georg; } \\
\text { Puppe,-Clemens }\end{array}$ & $\begin{array}{l}\text { Theory-and-Decision; } \\
41(1) \text {, July 1996, pp. } 37- \\
58 .\end{array}$ & 1996 & Kirchsteiger & Georg & Uni-Wien & 0.56 & 1 & 2 & 0.373333333 & \\
\hline $\begin{array}{l}\text { Involuntary Unemployment and Non- } \\
\text { compensating Wage Differentials in an } \\
\text { Experimental Labour Market }\end{array}$ & $\begin{array}{l}\text { Fehr,-Ernst; } \\
\text { Kirchsteiger,-Georg; } \\
\text { Riedl,-Arno }\end{array}$ & $\begin{array}{l}\text { Economic-Journal; } \\
\text { 106(434), January 1996, } \\
\text { pp. 106-21. }\end{array}$ & 1996 & Kirchsteiger & Georg & Uni-Wien & 1.21 & 1 & 3 & 0.605 & \\
\hline The Role of Envy in Ultimatum Games & Kirchsteiger,-Georg & $\begin{array}{l}\text { Journal-of-Economic- } \\
\text { Behavior-and- } \\
\text { Organization; 25(3), } \\
\text { December 1994, pp. 373- } \\
89 .\end{array}$ & 1994 & Kirchsteiger & Georg & Uni-Wien & 0.42 & 1 & 1 & 0.42 & \\
\hline $\begin{array}{l}\text { Insider Power, Wage Discrimination } \\
\text { and Fairness }\end{array}$ & \begin{tabular}{|l|} 
Fehr,-Ernst; \\
Kirchsteiger,-Georg
\end{tabular} & $\begin{array}{l}\text { Economic-Journal; } \\
\text { 104(424), May 1994, } \\
\text { pp. } 571-83 .\end{array}$ & 1994 & Kirchsteiger & Georg & Uni-Wien & 1.21 & 1 & 2 & 0.806666667 & \\
\hline $\begin{array}{l}\text { Does Fairness Prevent Market } \\
\text { Clearing? An Experimental } \\
\text { Investigation }\end{array}$ & \begin{tabular}{|l|} 
Fehr,-Ernst; \\
Kirchsteiger,-Georg, \\
Arno Riedl \\
\end{tabular} & $\begin{array}{l}\text { Quarterly Journal of } \\
\text { Economics }\end{array}$ & 1993 & Kirchsteiger & Georg & Uni-Wien & 3.2 & 1 & 3 & 1.6 & $\begin{array}{l}\text { G. Kirchsteiger: } \\
6.52\end{array}$ \\
\hline $\begin{array}{l}\text { On the Role of Seasonal Intercepts in } \\
\text { Seasonal Cointegration }\end{array}$ & $\begin{array}{l}\text { Franses,-Philip- } \\
\text { Hans; Kunst,-- } \\
\text { Robert-M. }\end{array}$ & $\begin{array}{l}\text { Oxford-Bulletin-of- } \\
\text { Economics-and-Statistics; } \\
61(3), \text { August 1999, } \\
\text { pp. 409-33. } \\
\end{array}$ & 1999 & Kunst & Robert & Uni-Wien & 0.76 & 1 & 2 & 0.506666667 & \\
\hline $\begin{array}{l}\text { Augmented ARCH Models for Financial } \\
\text { Time Series: Stability Conditions and } \\
\text { Empirical Evidence }\end{array}$ & Kunst,--Robert-M. & $\begin{array}{l}\text { Applied-Financial- } \\
\text { Economics; 7(6), } \\
\text { December 1997, pp. 575- } \\
86 .\end{array}$ & 1997 & Kunst & Robert & Uni-Wien & 0 & 1 & 1 & 0 & \\
\hline
\end{tabular}


Universität Wien

\begin{tabular}{|c|c|c|c|c|c|c|c|c|c|c|c|}
\hline & Author(s) & Journal & Year & & & & $\begin{array}{l}\text { Impact } \\
\text { Factor }\end{array}$ & $\begin{array}{l}\text { Page- } \\
\text { corr. }\end{array}$ & Authors & Impact & personal Impact \\
\hline $\begin{array}{l}\text { Fractionally Integrated Models with } \\
\text { ARCH Errors: With an Application to } \\
\text { the Swiss One-Month Euromarket } \\
\text { Interest Rate }\end{array}$ & $\begin{array}{l}\text { Hauser,-Michael-A.; } \\
\text { Kunst,-Robert-M. }\end{array}$ & $\begin{array}{l}\text { Review -of-Quantitative- } \\
\text { Finance-and-Accounting; } \\
\text { 10(1), January 1998, } \\
\text { pp. 95-113. }\end{array}$ & 1998 & Kunst & Robert & Uni-Wien & 0 & 1 & 2 & 0 & \\
\hline $\begin{array}{l}\text { Forecasting Seasonally Cointegrated } \\
\text { Systems: Supply Response of the } \\
\text { Austrian Breeding Sow Herd }\end{array}$ & $\begin{array}{l}\text { Jumah,-Adusei; } \\
\text { Kunst,-Robert-M. }\end{array}$ & $\begin{array}{l}\text { European-Review -of- } \\
\text { Agricultural-Economics; } \\
\text { 23(4), 1996, pp. 487-507. }\end{array}$ & 1996 & Kunst & Robert & Uni-Wien & 0.32 & 1 & 2 & 0.213333333 & \\
\hline $\begin{array}{l}\text { Modelling Exchange Rates: Long-Run } \\
\text { Dependence versus Conditional } \\
\text { Heteroscedasticity }\end{array}$ & $\begin{array}{l}\text { Hauser,-Michael-A.; } \\
\text { Kunst,-Robert-M.; } \\
\text { Reschenhofer,-- } \\
\text { Erhard } \\
\end{array}$ & $\begin{array}{l}\text { Applied-Financial- } \\
\text { Economics; 4(3), June } \\
\text { 1994, pp. 233-39. }\end{array}$ & 1994 & Kunst & Robert & Uni-Wien & 0 & 0.5 & 3 & 0 & \\
\hline $\begin{array}{l}\text { Seasonal Cointegration, Common } \\
\text { Seasonals, and Forecasting Seasonal } \\
\text { Series }\end{array}$ & Kunst,-Robert-M. & $\begin{array}{l}\text { Empirical-Economics; } \\
\text { 18(4), 1993, pp. 761-76. }\end{array}$ & 1993 & Kunst & Robert & Uni-Wien & 0 & 1 & 1 & 0 & \\
\hline $\begin{array}{l}\text { Seasonal Cointegration in } \\
\text { Macroeconomic Systems: Case } \\
\text { Studies for Small and Large European } \\
\text { Countries }\end{array}$ & Kunst,-Robert-M. & $\begin{array}{l}\text { Review-of-Economics- } \\
\text { and-Statistics; 75(2), May } \\
\text { 1993, pp. 325-30. }\end{array}$ & 1993 & Kunst & Robert & Uni-Wien & 1.04 & 0.5 & 1 & 0.52 & \\
\hline $\begin{array}{l}\text { Analysis of Austrian Stocks: Testing for } \\
\text { Stability and Randomness }\end{array}$ & \begin{tabular}{|l|} 
Kunst,--Robert-M.; \\
Reschenhofer,-- \\
Erhard; Rodler,-Kurt \\
\end{tabular} & $\begin{array}{l}\text { Empirical-Economics; } \\
\text { 16(4), 1991, pp. 465-77. } \\
\end{array}$ & 1991 & Kunst & Robert & Uni-Wien & 0 & 1 & 3 & 0 & \\
\hline $\begin{array}{l}\text { Cointegration in a Macroeconomic } \\
\text { System }\end{array}$ & \begin{tabular}{|l|} 
Kunst,-Robert; \\
Neusser,-Klaus
\end{tabular} & $\begin{array}{l}\text { Journal-of-Applied- } \\
\text { Econometrics; 5(4), Oct.- } \\
\text { Dec. 1990, pp. 351-65. } \\
\end{array}$ & 1990 & Kunst & Robert & Uni-Wien & 0.72 & 1 & 2 & 0.48 & \\
\hline $\begin{array}{l}\text { Seasonal Adjustment and Measuring } \\
\text { Persistence in Output }\end{array}$ & $\begin{array}{l}\text { Jaeger,-Albert; } \\
\text { Kunst,-Robert-M. }\end{array}$ & $\begin{array}{l}\text { Journal-of-Applied- } \\
\text { Econometrics; 5(1), } \\
\text { January-March 1990, } \\
\text { pp. 47-58. }\end{array}$ & 1990 & Kunst & Robert & Uni-Wien & 0.72 & 1 & 2 & 0.48 & R. Kunst: 2.2 \\
\hline $\begin{array}{l}\text { Are Two a Good Representative for } \\
\text { Many? }\end{array}$ & $\begin{array}{l}\text { Kerschbamer,- } \\
\text { Rudolf; Maderner,- } \\
\text { Nina }\end{array}$ & $\begin{array}{l}\text { Journal-of-Economic- } \\
\text { Theory; 83(1), November } \\
\text { 1998, pp. 90-104. }\end{array}$ & 1998 & Maderner & Nina & Uni-Wien & 0.93 & 1 & 2 & 0.62 & \\
\hline $\begin{array}{l}\text { Is It Legitimate to Encourage Work } \\
\text { Sharing? }\end{array}$ & $\begin{array}{l}\text { Maderner,-Nina; } \\
\text { Rochet,-Jean- } \\
\text { Charles }\end{array}$ & $\begin{array}{l}\text { Scandinavian-Journal-of- } \\
\text { Economics; 97(4), } \\
\text { December 1995, pp. 621- } \\
\text { 33. }\end{array}$ & 1995 & Maderner & Nina & Uni-Wien & 0.48 & 1 & 2 & 0.32 & N. Maderner: 0.94 \\
\hline $\begin{array}{l}\text { Roscher's Epistemological and } \\
\text { Methodological Position: Its } \\
\text { Importance for the Methodenstreit }\end{array}$ & Milford,-Karl & $\begin{array}{l}\text { Journal-of-Economic- } \\
\text { Studies; 22(3-4-5), 1995, } \\
\text { pp. 26-52. }\end{array}$ & 1995 & Milford & Karl & Uni-Wien & 0 & 1 & 1 & 0 & \\
\hline $\begin{array}{l}\text { Theoretical and Methodological } \\
\text { Positions of German Economics in the } \\
\text { Middle of the Nineteenth Century }\end{array}$ & $\begin{array}{l}\text { Streissler,-Erich; } \\
\text { Milford,-Karl }\end{array}$ & $\begin{array}{l}\text { History-of-Economic- } \\
\text { Ideas; } 1(-2 \text { 3-1), 1993- } \\
\text { 1994, pp. 43-79. } \\
\end{array}$ & 1993 & Milford & Karl & Uni-Wien & 0 & 1 & 2 & 0 & \\
\hline Menger's Methodology & Milford,-Karl & $\begin{array}{l}\text { History-of-Political- } \\
\text { Economy; 22(5), 1990, } \\
\text { pp. 215-39. }\end{array}$ & 1990 & Milford & Karl & Uni-Wien & 0.12 & 1 & 1 & 0.12 & K. Milford: 0.12 \\
\hline $\begin{array}{l}\text { Further Comment on the Social } \\
\text { Benefits from an Effective Antimerger } \\
\text { Policy }\end{array}$ & Mueller,-Dennis-C. & $\begin{array}{l}\text { Review -of-Industrial- } \\
\text { Organization; 12(5-6), } \\
\text { December 1997, pp. 701- } \\
02 .\end{array}$ & 1997 & Mueller & Dennis & Uni-Wien & 0 & 0.5 & 1 & 0 & \\
\hline
\end{tabular}


Universität Wien

\begin{tabular}{|c|c|c|c|c|c|c|c|c|c|c|c|}
\hline & Author(s) & Journal & Year & & & & $\begin{array}{l}\text { Impact } \\
\text { Factor }\end{array}$ & $\begin{array}{l}\text { Page- } \\
\text { corr. }\end{array}$ & Authors & Impact & personal Impact \\
\hline $\begin{array}{l}\text { Merger Policy in the United States: A } \\
\text { Reconsideration }\end{array}$ & Mueller,-Dennis-C. & $\begin{array}{l}\text { Review -of-Industrial- } \\
\text { Organization; 12(5-6), } \\
\text { December 1997, pp. 655- } \\
85 .\end{array}$ & 1997 & Mueller & Dennis & Uni-Wien & 0 & 1 & 1 & 0 & \\
\hline $\begin{array}{l}\text { Should the European Parliament Be } \\
\text { Given the Power to Elect the } \\
\text { Commission? }\end{array}$ & Mueller,-Dennis-C. & $\begin{array}{l}\text { Aussenwirtschaft; 52(1-2), } \\
\text { June 1997, pp. 249-53. }\end{array}$ & 1997 & Mueller & Dennis & Uni-Wien & 0 & 0.5 & 1 & 0 & \\
\hline $\begin{array}{l}\text { First-Mover Advantages and Path } \\
\text { Dependence }\end{array}$ & Mueller,-Dennis-C. & $\begin{array}{l}\text { International-Journal-of- } \\
\text { Industrial-Organization; } \\
\text { 15(6), October 1997, } \\
\text { pp. 827-50. }\end{array}$ & 1997 & Mueller & Dennis & Uni-Wien & 0.34 & 1 & 1 & 0.34 & \\
\hline $\begin{array}{l}\text { Business and Industrial Organization } \\
\text { Research: Synergy or Shotgun } \\
\text { Marriage? }\end{array}$ & Mueller,-Dennis-C. & $\begin{array}{l}\text { Journal-of-the-Economics- } \\
\text { of-Business; 1(1), 1994, } \\
\text { pp. } 27-30 .\end{array}$ & 1994 & Mueller & Dennis & Uni-Wien & 0 & 0.5 & 1 & 0 & \\
\hline $\begin{array}{l}\text { Managerial Discretion and Managerial } \\
\text { Compensation }\end{array}$ & $\begin{array}{l}\text { Mueller,-Dennis-C.; } \\
\text { Yun,-S.-Lawrence }\end{array}$ & $\begin{array}{l}\text { International-Journal-of- } \\
\text { Industrial-Organization; } \\
\text { 15(4), July 1997, pp. } 441 \text { - } \\
54 .\end{array}$ & 1997 & Mueller & Dennis & Uni-Wien & 0.34 & 1 & 2 & 0.226666667 & \\
\hline $\begin{array}{l}\text { Federalism and the European Union: A } \\
\text { Constitutional Perspective }\end{array}$ & Mueller,-Dennis-C. & $\begin{array}{l}\text { Public-Choice; 90(1-4), } \\
\text { March 1997, pp. 255-80. }\end{array}$ & 1997 & Mueller & Dennis & Uni-Wien & 0.43 & 1 & 1 & 0.43 & \\
\hline $\begin{array}{l}\text { Antimerger Policy in the United States: } \\
\text { History and Lessons }\end{array}$ & Mueller,-Dennis-C. & $\begin{array}{l}\text { Empirica; 23(3), 1996, } \\
\text { pp. 229-53. }\end{array}$ & 1996 & Mueller & Dennis & Uni-Wien & 0 & 1 & 1 & 0 & \\
\hline Constitutional Quandaries in Europe & Mueller,-Dennis-C. & $\begin{array}{l}\text { Constitutional-Political- } \\
\text { Economy; 7(4), 1996, } \\
\text { pp. 293-302. }\end{array}$ & 1996 & Mueller & Dennis & Uni-Wien & 0 & 0.5 & 1 & 0 & \\
\hline $\begin{array}{l}\text { Lessons from the United States's } \\
\text { Antitrust History }\end{array}$ & Mueller,-Dennis-C. & $\begin{array}{l}\text { International-Journal-of- } \\
\text { Industrial-Organization; } \\
\text { 14(4), June 1996, pp. 415- } \\
45 .\end{array}$ & 1996 & Mueller & Dennis & Uni-Wien & 0.34 & 1 & 1 & 0.34 & \\
\hline $\begin{array}{l}\text { Investment and Cash Flow: } \\
\text { Asymmetric Information or Managerial } \\
\text { Discretion }\end{array}$ & $\begin{array}{l}\text { Kathuria,-Rajat; } \\
\text { Mueller,-Dennis-C. }\end{array}$ & $\begin{array}{l}\text { Empirica; 22(3), 1995, } \\
\text { pp. 211-34. }\end{array}$ & 1995 & Mueller & Dennis & Uni-Wien & 0 & 1 & 2 & 0 & \\
\hline $\begin{array}{l}\text { Symposium on Investment and Cash } \\
\text { Flow: Introduction }\end{array}$ & Mueller,-Dennis-C. & $\begin{array}{l}\text { Empirica; 22(3), 1995, } \\
\text { pp. 163-66. }\end{array}$ & 1995 & Mueller & Dennis & Uni-Wien & 0 & 0.5 & 1 & 0 & \\
\hline $\begin{array}{l}\text { Parliamentary Systems in a } \\
\text { Heterogeneous Society }\end{array}$ & Mueller,-Dennis-C. & $\begin{array}{l}\text { Journal-of-Public-Finance- } \\
\text { and-Public-Choice/ } \\
\text { Economia-DelleScelte- } \\
\text { Pubbliche; 10(2-3), May- } \\
\text { Dec. 1992, pp. 119-36. }\end{array}$ & 1992 & Mueller & Dennis & Uni-Wien & 0 & 1 & 1 & 0 & \\
\hline $\begin{array}{l}\text { Federalisme et union europeenne: Une } \\
\text { perspective constitutionnelle. }\end{array}$ & Mueller,-Dennis-C. & $\begin{array}{l}\text { Revue-d'Economie- } \\
\text { Politique; 105(5), Sept.- } \\
\text { Oct. 1995, pp. 779-805. }\end{array}$ & 1995 & Mueller & Dennis & Uni-Wien & 0 & 1 & 1 & 0 & \\
\hline American and European Economists & Mueller,-Dennis-C. & $\begin{array}{l}\text { Kyklos; 48(2), 1995, } \\
\text { pp. 251-55. }\end{array}$ & 1995 & Mueller & Dennis & Uni-Wien & 0.37 & 0.5 & 1 & 0.185 & \\
\hline $\begin{array}{l}\text { Informative and Persuasive } \\
\text { Campaigning }\end{array}$ & $\begin{array}{l}\text { Mueller,-Dennis-C.; } \\
\text { Stratmann,-Thomas }\end{array}$ & $\begin{array}{l}\text { Public-Choice; 81(1-2), } \\
\text { October 1994, pp. 55-77. }\end{array}$ & 1994 & Mueller & Dennis & Uni-Wien & 0.43 & 1 & 2 & 0.286666667 & \\
\hline
\end{tabular}


Universität Wien

\begin{tabular}{|c|c|c|c|c|c|c|c|c|c|c|c|}
\hline & Author(s) & Journal & Year & & & & $\begin{array}{l}\text { Impact } \\
\text { Factor }\end{array}$ & $\begin{array}{l}\text { Page- } \\
\text { corr. }\end{array}$ & Authors & Impact & personal Impact \\
\hline $\begin{array}{l}\text { Rates of Return on Corporate } \\
\text { Investment }\end{array}$ & $\begin{array}{l}\text { Mueller,-Dennis-C.; } \\
\text { Reardon,-Elizabeth- } \\
\text { A. }\end{array}$ & $\begin{array}{l}\text { Southern-Economic- } \\
\text { Journal; 60(2), October } \\
\text { 1993, pp. 430-53. }\end{array}$ & 1993 & Mueller & Dennis & Uni-Wien & 0.39 & 1 & 2 & 0.26 & \\
\hline The Future of Public Choice & Mueller,-Dennis-C. & $\begin{array}{l}\text { Public-Choice; 77(1), } \\
\text { September 1993, pp. 145- } \\
\text { 50. }\end{array}$ & 1993 & Mueller & Dennis & Uni-Wien & 0.43 & 0.5 & 1 & 0.215 & \\
\hline $\begin{array}{l}\text { U.S. Merger Policy and the } 1992 \\
\text { Merger Guidelines }\end{array}$ & Mueller,-Dennis-C. & $\begin{array}{l}\text { Review -of-Industrial- } \\
\text { Organization; 8(2), 1993, } \\
\text { pp. 151-62. }\end{array}$ & 1993 & Mueller & Dennis & Uni-Wien & 0 & 1 & 1 & 0 & \\
\hline $\begin{array}{l}\text { James M. Buchanan: Economist cum } \\
\text { Contractarian }\end{array}$ & Mueller,-Dennis-C. & $\begin{array}{l}\text { Constitutional-Political- } \\
\text { Economy; 1(2), Spring- } \\
\text { Summer 1990, pp. 169- } \\
96 .\end{array}$ & 1990 & Mueller & Dennis & Uni-Wien & 0 & 1 & 1 & 0 & \\
\hline $\begin{array}{l}\text { Deregulation in Retrospect: } \\
\text { Symposium }\end{array}$ & Mueller,-Dennis-C. & $\begin{array}{l}\text { Southern-Economic- } \\
\text { Journal; 59(3), January } \\
1993, \text { pp. 436-37. }\end{array}$ & 1993 & Mueller & Dennis & Uni-Wien & 0.39 & 0.5 & 1 & 0.195 & \\
\hline The Corporation and the Economist & Mueller,-Dennis-C. & $\begin{array}{l}\text { International-Journal-of- } \\
\text { Industrial-Organization; } \\
\text { 10(2), June 1992, pp. 147- } \\
70 .\end{array}$ & 1992 & Mueller & Dennis & Uni-Wien & 0.34 & 1 & 1 & 0.34 & \\
\hline Profit Rates and Intangible Capital & $\begin{array}{l}\text { Megna,-Pamela; } \\
\text { Mueller,-Dennis-C. }\end{array}$ & $\begin{array}{l}\text { Review -of-Economics- } \\
\text { and-Statistics; 73(4), } \\
\text { November 1991, pp. 632- } \\
42 .\end{array}$ & 1991 & Mueller & Dennis & Uni-Wien & 1.04 & 1 & 2 & 0.693333333 & \\
\hline Constitutional Rights & Mueller,-Dennis-C. & $\begin{array}{l}\text { Journal-of-Law,- } \\
\text { Economics-and- } \\
\text { Organization; 7(2), Fall } \\
\text { 1991, pp. 313-33. }\end{array}$ & 1991 & Mueller & Dennis & Uni-Wien & 1.12 & 1 & 1 & 1.12 & \\
\hline $\begin{array}{l}\text { Choosing a Constitution in East } \\
\text { Europe: Lessons from Public Choice }\end{array}$ & Mueller,-Dennis-C. & $\begin{array}{l}\text { Journal-of-Comparative- } \\
\text { Economics; } 15(2), \text { June } \\
\text { 1991, pp. 325-48. }\end{array}$ & 1991 & Mueller & Dennis & Uni-Wien & 1.27 & 1 & 1 & 1.27 & \\
\hline $\begin{array}{l}\text { Electoral Politics, Interest Groups, and } \\
\text { the Size of Government }\end{array}$ & $\begin{array}{l}\text { Coughlin,-Peter-J.; } \\
\text { Mueller,-Dennis-C.; } \\
\text { Murrell,-Peter }\end{array}$ & $\begin{array}{l}\text { Economic-Inquiry; 28(4), } \\
\text { October 1990, pp. 682- } \\
705 .\end{array}$ & 1990 & Mueller & Dennis & Uni-Wien & 0.62 & 1 & 3 & 0.31 & \\
\hline $\begin{array}{l}\text { A Model of Electoral Competition with } \\
\text { Interest Groups }\end{array}$ & $\begin{array}{l}\text { Coughlin,-Peter-J.; } \\
\text { Mueller,-Dennis-C.; } \\
\text { Murrell,-Peter }\end{array}$ & $\begin{array}{l}\text { Economics-Letters; 32(4), } \\
\text { April 1990, pp. 307-11. }\end{array}$ & 1990 & Mueller & Dennis & Uni-Wien & 0.26 & 0.5 & 3 & 0.065 & $\begin{array}{l}\text { D. Mueller: } \\
\text { 6.276666667 }\end{array}$ \\
\hline $\begin{array}{l}\text { Sensitivity of Optimal Growth Paths } \\
\text { with ...: A Corrigendum }\end{array}$ & Nermuth,-Manfred & $\begin{array}{l}\text { Journal-of-Mathematical- } \\
\text { Economics; 22(4), 1993, } \\
\text { pp. 399-402. }\end{array}$ & 1993 & Nermuth & Manfred & Uni-Wien & 0.37 & 0.5 & 1 & 0.185 & \\
\hline $\begin{array}{l}\text { Two-Stage Discrete Aggregation: The } \\
\text { Ostrogorski Paradox and Related } \\
\text { Phenomena }\end{array}$ & Nermuth,-Manfred & $\begin{array}{l}\text { Social-Choice-and- } \\
\text { Welfare; } 9(2) \text {, April 1992, } \\
\text { pp. 99-116. }\end{array}$ & 1992 & Nermuth & Manfred & Uni-Wien & 0.55 & 1 & 1 & 0.55 & M. Nermuth: 0.735 \\
\hline $\begin{array}{l}\text { Herding and the Winner's Curse in } \\
\text { Markets with Sequential Bids }\end{array}$ & $\begin{array}{l}\text { Neeman,-Zvika; } \\
\text { Orosel,-Gerhard-O. }\end{array}$ & $\begin{array}{l}\text { Journal-of-Economic- } \\
\text { Theory; 85(1), March } \\
\text { 1999, pp. } 91-121 .\end{array}$ & 1999 & Orosel & Gerhard & Uni-Wien & 0.93 & 1 & 2 & 0.62 & \\
\hline
\end{tabular}


Universität Wien

\begin{tabular}{|c|c|c|c|c|c|c|c|c|c|c|c|}
\hline & Author(s) & Journal & Year & & & & $\begin{array}{l}\text { Impact } \\
\text { Factor }\end{array}$ & $\begin{array}{l}\text { Page- } \\
\text { corr. }\end{array}$ & Authors & Impact & personal Impact \\
\hline $\begin{array}{l}\text { Participation Costs, Trend Chasing, and } \\
\text { Volatility of Stock Prices }\end{array}$ & Orosel,-Gerhard-O. & $\begin{array}{l}\text { Review -of-Financial- } \\
\text { Studies; 11(3), Fall 1998, } \\
\text { pp. 521-57. }\end{array}$ & 1998 & Orosel & Gerhard & Uni-Wien & 1.13 & 1 & 1 & 1.13 & \\
\hline $\begin{array}{l}\text { The Informational Role of Volume Vis- } \\
\text { a-Vis No-Trade Results: A Note }\end{array}$ & $\begin{array}{l}\text { Hsu,-Chiente; } \\
\text { Orosel,-Gerhard-O. }\end{array}$ & $\begin{array}{l}\text { Schweizerische- } \\
\text { Zeitschrift-fur- } \\
\text { Volkswirtschaft- } \\
\text { undStatistik; 134(2), June } \\
\text { 1998, pp. 133-58. }\end{array}$ & 1998 & Orosel & Gerhard & Uni-Wien & 0 & 1 & 2 & 0 & \\
\hline $\begin{array}{l}\text { Internalizing Externalities in Second- } \\
\text { Best Tax Systems }\end{array}$ & $\begin{array}{l}\text { Orosel,-Gerhard-O.; } \\
\text { Schob,-Ronnie }\end{array}$ & $\begin{array}{l}\text { Public-Finance; } 51(2) \\
1996, \text { pp. } 242-57 .\end{array}$ & 1996 & Orosel & Gerhard & Uni-Wien & 0 & 1 & 2 & 0 & \\
\hline Market Participation and Share Prices & Orosel,-Gerhard-O. & $\begin{array}{l}\text { Mathematical-Finance; } \\
\text { 7(4), October 1997, } \\
\text { pp. 375-98. }\end{array}$ & 1997 & Orosel & Gerhard & Uni-Wien & 0 & 1 & 1 & 0 & \\
\hline $\begin{array}{l}\text { Informational Efficiency and Welfare in } \\
\text { the Stock Market }\end{array}$ & Orosel,-Gerhard-O. & $\begin{array}{l}\text { European-Economic- } \\
\text { Review; 40(7), August } \\
\text { 1996, pp. 1379-1411. }\end{array}$ & 1996 & Orosel & Gerhard & Uni-Wien & 0.84 & 1 & 1 & 0.84 & \\
\hline $\begin{array}{l}\text { Inheritance and Inequality when Wealth } \\
\text { Enters the Utility Function }\end{array}$ & Orosel,-Gerhard-O. & $\begin{array}{l}\text { Journal-of-Economics- } \\
\text { (Zeitschrift-fur- } \\
\text { Nationalokonomie); 53(2), } \\
\text { 1991, pp. 133-60. }\end{array}$ & 1991 & Orosel & Gerhard & Uni-Wien & 0.18 & 1 & 1 & 0.18 & G. Orosel: 2.77 \\
\hline $\begin{array}{l}\text { Markets with Infinitely Many } \\
\text { Commodities and a Continuum of } \\
\text { Agents with Non-convex Preferences }\end{array}$ & Podczeck,-Konrad & $\begin{array}{l}\text { Economic-Theory; 9(3), } \\
\text { 1997, pp. 385-426. }\end{array}$ & 1997 & Podczeck & Konrad & Uni-Wien & 0 & 1 & 1 & 0 & \\
\hline $\begin{array}{l}\text { Adverse Selection and Regulation in } \\
\text { Health Insurance Markets }\end{array}$ & $\begin{array}{l}\text { Neudeck,-Werner; } \\
\text { Podczeck,-Konrad }\end{array}$ & $\begin{array}{l}\text { Journal-of-Health- } \\
\text { Economics; 15(4), August } \\
\text { 1996, pp. 387-408. }\end{array}$ & 1996 & Podczeck & Konrad & Uni-Wien & 1.39 & 1 & 2 & 0.926666667 & \\
\hline $\begin{array}{l}\text { Equilibria in Vector Lattices without } \\
\text { Ordered Preferences or Uniform } \\
\text { Properness }\end{array}$ & Podczeck,-Konrad & $\begin{array}{l}\text { Journal-of-Mathematical- } \\
\text { Economics; 25(4), May } \\
\text { 1996, pp. 465-85. }\end{array}$ & 1996 & Podczeck & Konrad & Uni-Wien & 0.37 & 1 & 1 & 0.37 & \\
\hline $\begin{array}{l}\text { General Equilibrium with Differentiated } \\
\text { Commodities: The Linear Activity } \\
\text { Model without Joint Production }\end{array}$ & Podczeck,-Konrad & $\begin{array}{l}\text { Economic-Theory; 2(2), } \\
\text { April 1992, pp. 247-63. }\end{array}$ & 1992 & Podczeck & Konrad & Uni-Wien & 0 & 1 & 1 & 0 & $\begin{array}{l}\text { K. Podceck: } \\
1.296666667\end{array}$ \\
\hline $\begin{array}{l}\text { Neoliberalism and Globalisation-- } \\
\text { Justifying Policies of Redistribution }\end{array}$ & Raffer,-Kunibert & $\begin{array}{l}\text { Indian-Journal-of-Applied- } \\
\text { Economics; 8(1), Jan.- } \\
\text { March 1999, pp. 191-214. }\end{array}$ & 1999 & Raffer & Kunibert & Uni-Wien & 0 & 1 & 1 & 0 & \\
\hline $\begin{array}{l}\text { 'Looking a Gift Horse in the Mouth': } \\
\text { Analysing Donors' Aid Statistics }\end{array}$ & Raffer,-Kunibert & $\begin{array}{l}\text { Zagreb-International- } \\
\text { Review-of-Economics- } \\
\text { and-Business; 1(2), } \\
\text { November 1998, pp. 1-20. }\end{array}$ & 1998 & Raffer & Kunibert & Uni-Wien & 0 & 1 & 1 & 0 & \\
\hline The Tobin Tax: Reviving a Discussion & Raffer,-Kunibert & $\begin{array}{l}\text { World-Development; } \\
\text { 26(3), March 1998, } \\
\text { pp. 529-38. }\end{array}$ & 1998 & Raffer & Kunibert & Uni-Wien & 0.76 & 0.5 & 1 & 0.38 & \\
\hline $\begin{array}{l}\text { Helping Southern Net Food Importers } \\
\text { after the Uruguay Round: A Proposal }\end{array}$ & Raffer,-Kunibert & $\begin{array}{l}\text { World-Development; } \\
\text { 25(11), November } 1997, \\
\text { pp. 1901-07. }\end{array}$ & 1997 & Raffer & Kunibert & Uni-Wien & 0.76 & 0.5 & 1 & 0.38 & \\
\hline $\begin{array}{l}\text { Applying Chapter } 9 \text { Insolvency to } \\
\text { International Debts: An Economically } \\
\text { Efficient Solution with a Human Face }\end{array}$ & Raffer,-Kunibert & $\begin{array}{l}\text { World-Development; } \\
\text { 18(2), February 1990, } \\
\text { pp. 301-11. }\end{array}$ & 1990 & Raffer & Kunibert & Uni-Wien & 0.76 & 1 & 1 & 0.76 & K. Raffer: 1.52 \\
\hline
\end{tabular}


Universität Wien

\begin{tabular}{|c|c|c|c|c|c|c|c|c|c|c|c|}
\hline & Author(s) & Journal & Year & & & & $\begin{array}{l}\text { Impact } \\
\text { Factor }\end{array}$ & $\begin{array}{l}\text { Page- } \\
\text { corr. }\end{array}$ & Authors & Impact & personal Impact \\
\hline $\begin{array}{l}\text { Can We Consider the Keynesian } \\
\text { Revolution to Be Scientific Progress? }\end{array}$ & Rosner,-Peter & $\begin{array}{l}\text { History-of-Economics- } \\
\text { Review; 0(25), Winter- } \\
\text { Summer 1996, pp. 32-44. }\end{array}$ & 1996 & Rosner & Peter & Uni-Wien & 0 & 1 & 1 & 0 & \\
\hline $\begin{array}{l}\text { Say's Law and the Post-Napoleonic } \\
\text { Crisis }\end{array}$ & Rosner,-Peter & $\begin{array}{l}\text { History-of-Economic- } \\
\text { Ideas; 3(3), 1995, pp. 35- } \\
64 .\end{array}$ & 1995 & Rosner & Peter & Uni-Wien & 0 & 1 & 1 & 0 & P. Rosner: 0 \\
\hline $\begin{array}{l}\text { Increasing Returns to Scale and the } \\
\text { Prospects of Small-Scale Enterprises }\end{array}$ & Streissler,-Erich-W. & $\begin{array}{l}\text { Journal-of-Economic- } \\
\text { Studies; } 22(3-4-5), 1995, \\
\text { pp. 16-25. }\end{array}$ & 1995 & Streißler & Erich & Uni-Wien & 0 & 0.5 & 1 & 0 & \\
\hline $\begin{array}{l}\text { Theoretical and Methodological } \\
\text { Positions of German Economics in the } \\
\text { Middle of the Nineteenth Century }\end{array}$ & $\begin{array}{l}\text { Streissler,-Erich; } \\
\text { Milford,-Karl }\end{array}$ & $\begin{array}{l}\text { History-of-Economic- } \\
\text { Ideas; } 1(-2 \text { 3-1), 1993- } \\
\text { 1994, pp. 43-79. }\end{array}$ & 1993 & Streißler & Erich & Uni-Wien & 0 & 1 & 2 & 0 & \\
\hline $\begin{array}{l}\text { Exchange-Rate Systems, Currency } \\
\text { Unions, and Convertibility: Hungary's } \\
\text { Prospects }\end{array}$ & Streissler,-Erich-W. & $\begin{array}{l}\text { Russian-and-East- } \\
\text { European-Finance-and- } \\
\text { Trade; 30(4), July-Aug. } \\
\text { 1994, pp. 70-88. }\end{array}$ & 1994 & Streißler & Erich & Uni-Wien & 0 & 1 & 1 & 0 & \\
\hline $\begin{array}{l}\text { Genius or Engines? On Jurg Niehans' } \\
\text { History of Economic Technique }\end{array}$ & Streissler,-Erich-W. & $\begin{array}{l}\text { Journal-of-Institutional- } \\
\text { and-Theoretical- } \\
\text { Economics; } 147(2), \text { June } \\
\text { 1991, pp. 379-95. }\end{array}$ & 1991 & Streißler & Erich & Uni-Wien & 0.29 & 1 & 1 & 0.29 & \\
\hline $\begin{array}{l}\text { Carl Menger on Economic Policy: The } \\
\text { Lectures to Crown Prince Rudolf }\end{array}$ & Streissler,-Erich-W. & $\begin{array}{l}\text { History-of-Political- } \\
\text { Economy; 22(5), 1990, } \\
\text { pp. 107-30. } \\
\end{array}$ & 1990 & Streißler & Erich & Uni-Wien & 0.12 & 1 & 1 & 0.12 & \\
\hline $\begin{array}{l}\text { The Influence of German Economics on } \\
\text { the Work of Menger and Marshall }\end{array}$ & Streissler,-Erich-W. & $\begin{array}{l}\text { History-of-Political- } \\
\text { Economy; 22(5), 1990, } \\
\text { pp. 31-68. }\end{array}$ & 1990 & Streißler & Erich & Uni-Wien & 0.12 & 1 & 1 & 0.12 & E. Streißler: 0.53 \\
\hline $\begin{array}{l}\text { How Well Are Authorities Suited to } \\
\text { Prevent Damage to the Environment? }\end{array}$ & Weigel,-Wolfgang & $\begin{array}{l}\text { European-Journal-of-Law- } \\
\text { and-Economics; 4(1), } \\
\text { March 1997, pp. 23-39. }\end{array}$ & 1997 & Weigel & Wolfgang & Uni-Wien & 0 & 1 & 1 & 0 & \\
\hline $\begin{array}{l}\text { Prospects for Law and Economics in } \\
\text { Civil Law Countries: Austria }\end{array}$ & Weigel,-Wolfgang & $\begin{array}{l}\text { International-Review -of- } \\
\text { Law -and-Economics; } \\
\text { 11(3), December 1991, } \\
\text { pp. 325-29. }\end{array}$ & 1991 & Weigel & Wolfgang & Uni-Wien & 0 & 0.5 & 1 & 0 & W. Weigel: 0 \\
\hline $\begin{array}{l}\text { The Advantages of Tying Austria's } \\
\text { Hands: The Success of the Hard } \\
\text { Currency Strategy }\end{array}$ & $\begin{array}{l}\text { Hochreiter,-Eduard; } \\
\text { Winckler,-Georg }\end{array}$ & $\begin{array}{l}\text { European-Journal-of- } \\
\text { Political-Economy; 11(1), } \\
\text { March 1995, pp. 83-111. }\end{array}$ & 1995 & Winckler & Georg & Uni-Wien & 0 & $\overline{1}$ & 2 & 0 & \\
\hline $\begin{array}{l}\text { Central Banks and Seigniorage: A } \\
\text { Study of Three Economies in Transition }\end{array}$ & $\begin{array}{l}\text { Hochreiter,-Eduard; } \\
\text { Rovelli,-Riccardo; } \\
\text { Winckler,-Georg } \\
\end{array}$ & $\begin{array}{l}\text { European-Economic- } \\
\text { Review; 40(3-5), April } \\
\text { 1996, pp. 629-43. } \\
\end{array}$ & 1996 & Winckler & Georg & Uni-Wien & 0.84 & 1 & 3 & 0.42 & G. Winckler: 0.42 \\
\hline
\end{tabular}




\section{Wirtschaftsforschungsinstitut}

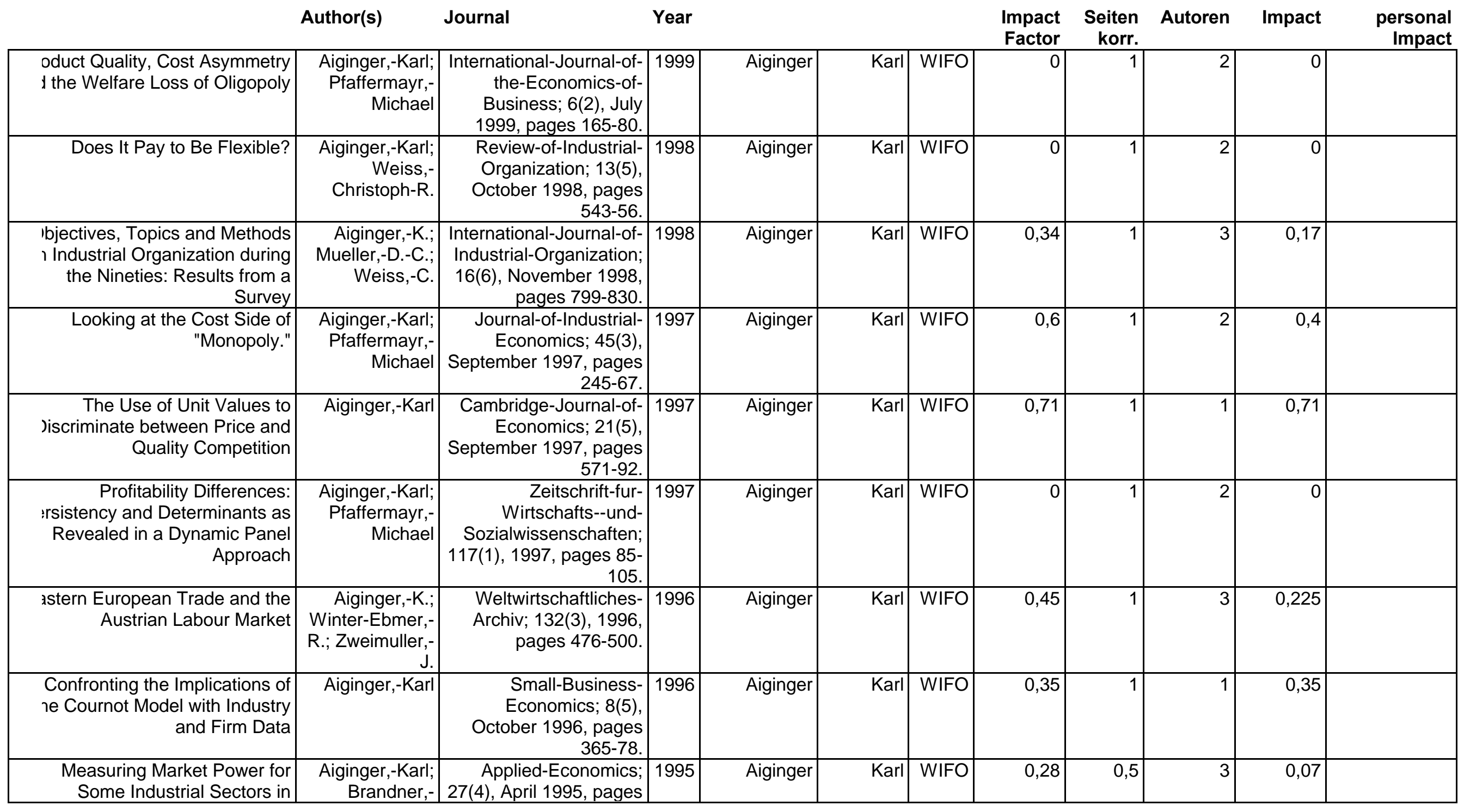




\begin{tabular}{|c|c|c|c|c|c|c|c|c|c|c|c|}
\hline Austria & $\begin{array}{r}\text { Peter; Wuger,- } \\
\text { Michael }\end{array}$ & $369-76$. & & & & & & & & & \\
\hline $\begin{array}{r}\text { The Explanatory Power of } \\
\text { arket-Based Trade Theories for } \\
\text { the Trade between Market } \\
\text { onomies and Reform Countries }\end{array}$ & $\begin{array}{r}\text { Aiginger,-K.; } \\
\text { Peneder,-M.; } \\
\text { Stankovsky,-J. }\end{array}$ & $\begin{array}{r}\text { Empirica; } 21(2), 1994 \\
\text { pages } 197-220 .\end{array}$ & 1994 & Aiginger & Karl & WIFO & 0 & 1 & 3 & 0 & \\
\hline $\begin{array}{r}\text { Small Firms and the Merger } \\
\text { Mania: Reply }\end{array}$ & Aiginger,-Karl & $\begin{array}{r}\text { Small-Business- } \\
\text { Economics; 5(4), } \\
\text { December 1993, pages } \\
323-30 .\end{array}$ & 1993 & Aiginger & Karl & WIFO & 0,35 & 0,5 & 1 & 0,175 & $\begin{array}{r}\text { K. Aiginger: } \\
2,1\end{array}$ \\
\hline $\begin{array}{r}\text { Collusion, Concentration and } \\
\text { Profits: An Empirical } \\
\text { nfrontation of an Old Story and } \\
\text { a Supergame Implication }\end{array}$ & Aiginger,-Karl & $\begin{array}{r}\text { Empirica; } 20(2), 1993 \\
\text { pages } 159-73 .\end{array}$ & 1993 & Aiginger & Karl & WIFO & 0 & 1 & 1 & 0 & \\
\hline $\begin{array}{r}\text { Injectural-Variation Models and } \\
\text { Supergames with Price } \\
\text { Competition in a Differentiated } \\
\text { Product Oligopoly }\end{array}$ & $\begin{array}{r}\text { Pfaffermayr,-- } \\
\text { Michael }\end{array}$ & $\begin{array}{r}\text { Journal-of-Economics; } \\
70(3), \text { 1999, pages 309- } \\
26 .\end{array}$ & 1999 & Pfaffermayr & Michael & WIFO & 0,18 & 1 & 1 & 0,18 & \\
\hline $\begin{array}{r}\text { whership Advantages, Foreign } \\
\text { Production and Productivity: } \\
\text { Evidence from Austrian } \\
\text { Manufacturing Firms }\end{array}$ & Pfaffermayr & $\begin{array}{r}\text { Review of Industrial } \\
\text { Organization; } 15(4), \\
\text { December 1999, pages } \\
379-96 .\end{array}$ & 1999 & Pfaffermayr & Michael & WIFO & 0 & 1 & 1 & 0 & \\
\hline $\begin{array}{l}\text { Troduct Quality, Cost Asymetry } \\
\text { 1 the Welfare Loss of Oligopoly }\end{array}$ & $\begin{array}{r}\text { Aiginger, } \\
\text { Pfaffermayr }\end{array}$ & $\begin{array}{r}\text { International Journal of } \\
\text { the Economics of } \\
\text { Business; } 6(2), \text { July } \\
\text { 1999, pages 165-80. }\end{array}$ & 1999 & Pfaffermayr & Michael & WIFO & 0 & 1 & 2 & 0 & \\
\hline $\begin{array}{r}\text { Multinationals, Production } \\
\text { Externalities, and } \\
\text { Complementarities between } \\
\text { Jomestic and Foreign Activities }\end{array}$ & Pfaffermayr & $\begin{array}{r}\text { Swiss Journal of } \\
\text { Economics; } 133(4), \\
\text { December 1997, pages } \\
673-89 .\end{array}$ & 1997 & Pfaffermayr & Michael & WIFO & 0 & 1 & 1 & 0 & \\
\hline $\begin{array}{r}\text { Looking at the Cost Side of } \\
\text { Monopoly }\end{array}$ & $\begin{array}{r}\text { Aiginger, } \\
\text { Pfaffermayr }\end{array}$ & $\begin{array}{r}\text { Jounal of Industrial } \\
\text { Organization; 45(3), } \\
\text { September 1997, pages } \\
245-67 .\end{array}$ & 1997 & Pfaffermayr & Michael & WIFO & 0,6 & 1 & 2 & 0,4 & \\
\hline $\begin{array}{r}\text { Profitability Differences: } \\
\text { irsistency and Determinants as } \\
\text { Revealed in a Dynamic Panel }\end{array}$ & $\begin{array}{r}\text { Aiginger, } \\
\text { Pfaffermayr }\end{array}$ & $\begin{array}{r}\text { Zeitschrift-fur- } \\
\text { Wirtschafts-und- } \\
\text { Sozialwissenschaften; } \\
117(1), 1997 \text {, pages } 85- \\
105 .\end{array}$ & 1997 & Pfaffermayr & Michael & WIFO & 0 & 1 & 2 & 0 & \\
\hline
\end{tabular}




\begin{tabular}{|c|c|c|c|c|c|c|c|c|c|c|c|}
\hline $\begin{array}{r}\text { Production, Foreign Production } \\
\text { and Exports of Direct Investing } \\
\text { Firms: Some Evidence from } \\
\text { Austria }\end{array}$ & & pages 317-28. & & & & & & & & & \\
\hline $\begin{array}{r}\text { Technological Competition and } \\
\text { Trade Performance }\end{array}$ & $\begin{array}{r}\text { Landesmann, } \\
\text { Pfaffermayr }\end{array}$ & $\begin{array}{r}\text { Applied Economics; } \\
29(2), \text { February } 1997, \\
\text { pages } 179-96 . \\
\end{array}$ & 1997 & Pfaffermayr & Michael & WIFO & 0,28 & 1 & 2 & 0,186666 & \\
\hline $\begin{array}{r}\text { Foreign Outward Direct } \\
\text { Investment and Exports in } \\
\text { Austrian Manufacturing: } \\
\text { Substitutes or Complements }\end{array}$ & Pfaffermayr & $\begin{array}{r}\text { Weltwirtschaftliches- } \\
\text { Archiv; } 132(3), 1996, \\
\text { pages } 501-22 .\end{array}$ & 1996 & Pfaffermayr & Michael & WIFO & 0,45 & 1 & 1 & 0,45 & \\
\hline $\begin{array}{l}\text { Foreign Direct Investment and } \\
\text { ports: A Time Series Approach }\end{array}$ & Pfaffermayr & $\begin{array}{r}\text { Applied Economics; } \\
\text { 26(4), April 1994, pages } \\
337-51 .\end{array}$ & 1994 & Pfaffermayr & Michael & WIFO & 0,28 & 1 & 1 & 0,28 & \\
\hline $\begin{array}{r}\text { rm Income, Market Wages and } \\
\text { Off-Farm Labor Supply }\end{array}$ & $\begin{array}{r}\text { Pfaffermayr, } \\
\text { Weiss, } \\
\text { Zweimueller }\end{array}$ & $\begin{array}{r}\text { Empirica; 18(2), 1991, } \\
\text { pages 221-35. }\end{array}$ & 1991 & Pfaffermayr & Michael & WIFO & 0 & 1 & 3 & 0 & $\begin{array}{r}\mathrm{M} . \\
\text { Pfaffermayr: } \\
1,496 \\
\end{array}$ \\
\hline $\begin{array}{r}\text { Vachstum und Arbeitslosigkeit. } \\
\text { Neue Erkenntnisse aus der } \\
\text { Wachstumstheorie }\end{array}$ & Hahn,-Franz-R. & $\begin{array}{r}\text { Ifo-Studien; 40(4), 1994, } \\
\text { pages 305-19. }\end{array}$ & 1994 & Hahn & Franz & WIFO & 0 & 1 & 1 & 0 & \\
\hline $\begin{array}{l}\text { Business Cycles in Open } \\
\text { Economies: A Comment }\end{array}$ & Hahn,-Franz-R. & $\begin{array}{r}\text { Weltwirtschaftliches- } \\
\text { Archiv; } 130(3), 1994, \\
\text { pages 624-29. }\end{array}$ & 1994 & Hahn & Franz & WIFO & 0,45 & 0,5 & 1 & 0,225 & F. Hahn: \\
\hline $\begin{array}{r}\text { uilibrium Dynamics and Chaos: } \\
\text { A Textbook Approach }\end{array}$ & Hahn,-Franz-R. & $\begin{array}{r}\text { Jahrbucher-fur- } \\
\text { Nationalokonomie-und- } \\
\text { Statistik; 210(3-4), Sept. } \\
\text { 1992, pp. 279-85. }\end{array}$ & 1992 & Hahn & Franz & WIFO & 0,21 & 0,5 & 1 & 0,105 & 0,33 \\
\hline $\begin{array}{r}\text { Macroeconomic and Sectoral } \\
\text { Effects of Energy Taxation in } \\
\text { Austria }\end{array}$ & Koeppl,-Angela & $\begin{array}{r}\text { Environmental-and- } \\
\text { Resource-Economics; } \\
\text { 8(4), December 1996, } \\
\text { pages 417-30. }\end{array}$ & 1996 & Köppl & Angela & WIFO & 0 & 1 & 1 & 0 & A. Köppl: 0 \\
\hline $\begin{array}{r}\text { bate on the Transition of Post- } \\
\text { Communist Economies to a } \\
\text { Market Economy }\end{array}$ & Kramer,-Helmut & $\begin{array}{r}\text { Acta-Oeconomica; } \\
44(3-4), 1992, \text { pages } \\
318-21 . \\
\end{array}$ & 1992 & Kramer & Helmut & WIFO & 0 & 0,5 & 1 & 0 & H. Kramer: \\
\hline $\begin{array}{r}\text { Istria's Economic Perspectives } \\
\text { in the New Central-European } \\
\text { Environment }\end{array}$ & Kramer,-Helmut & $\begin{array}{r}\text { Acta-Oeconomica; } \\
42(3-4), 1990, \text { pages } \\
217-22 .\end{array}$ & 1990 & Kramer & Helmut & WIFO & 0 & 0,5 & 1 & 0 & 0 \\
\hline $\begin{array}{r}\text { Impact of Carbon Dioxide } \\
\text { Emissions Reduction on the } \\
\text { Austrian Economy }\end{array}$ & $\begin{array}{r}\text { Kratena,-Kurt; } \\
\text { Schleicher,- } \\
\text { Stefan }\end{array}$ & $\begin{array}{r}\text { Economic-Systems- } \\
\text { Research; 11(3), } \\
\text { September 1999, pages }\end{array}$ & 1999 & Kratena & Kurt & WIFO & 0 & 1 & 2 & 0 & K. Kratena: \\
\hline
\end{tabular}




\begin{tabular}{|c|c|c|c|c|c|c|c|c|c|c|c|}
\hline & & $245-61$. & & & & & & & & & \\
\hline $\begin{array}{r}\text { Produktion, Umweltpolitik und } \\
\text { Einkommensverteilung. Ein } \\
\text { umweltokonomischer Ansatz in } \\
\text { der Tradition von Ricardo und } \\
\text { Sraffa. (Production, } \\
\text { vironmental Policy and Income } \\
\text { istribution: A New Approach in } \\
\text { nvironmental Economics in the } \\
\text { Tradition of Ricardo } \\
\end{array}$ & Kratena,-Kurt & $\begin{array}{r}\text { Jahrbucher-fur- } \\
\text { Nationalokonomie-und- } \\
\text { Statistik; 207(5), Sept. } \\
\text { 1990, pp. 417-31. }\end{array}$ & 1990 & Kratena & Kurt & WIFO & 0,21 & 1 & 1 & 0,21 & 0,21 \\
\hline $\begin{array}{r}\text { Determinants of Product and } \\
\text { Process Innovation }\end{array}$ & Leo,-Hannes & $\begin{array}{r}\text { Economies-et-Societes; } \\
30(7), \text { July 1996, pages } \\
61-77\end{array}$ & 1996 & Leo & Hannes & WIFO & 0 & 1 & 1 & 0 & H. Leo: \\
\hline $\begin{array}{r}\text { Transfer Sciences and the } \\
\text { Austrian System of Innovation }\end{array}$ & Leo,-Hannes & $\begin{array}{r}\text { Empirica; } 21(3), 1994 \\
\text { pages } 271-83 \\
\end{array}$ & 1994 & Leo & Hannes & WIFO & 0 & 1 & 1 & 0 & 0 \\
\hline $\begin{array}{r}\text { Iuster Techniques as a Method } \\
\text { to Analyze Industrial } \\
\text { Competitiveness }\end{array}$ & $\begin{array}{r}\text { Peneder,- } \\
\text { Michael }\end{array}$ & $\begin{array}{r}\text { International-Advances- } \\
\text { in-Economic-Research; } \\
\text { 1(3), August 1995, } \\
\text { pages 295-303. }\end{array}$ & 1995 & Peneder & Michael & WIFO & 0 & 0,5 & 1 & 0 & M. Peneder: \\
\hline $\begin{array}{r}\text { The Equivalence of a } \\
\text { oductivity-Geared Wage Policy } \\
\text { and the Scandinavian Model of } \\
\text { Inflation }\end{array}$ & $\begin{array}{r}\text { Pollan,-- } \\
\text { Wolfgang }\end{array}$ & $\begin{array}{r}\text { Jahrbucher-fur- } \\
\text { Nationalokonomie-und- } \\
\text { Statistik; 208(3), May } \\
\text { 1991, pages 254-61. }\end{array}$ & 1991 & Pollan & Wolfgang & WIFO & 0,21 & 0,5 & 1 & 0,105 & W. Pollan: \\
\hline $\begin{array}{r}\text { Wage Norms in an Open } \\
\text { Economy }\end{array}$ & $\begin{array}{r}\text { Pollan,- } \\
\text { Wolfgang } \\
\end{array}$ & $\begin{array}{r}\text { Empirica; } 17(2), 1990 \\
\text { pages 187-99. }\end{array}$ & 1990 & Pollan & Wolfgang & WIFO & 0 & 1 & 1 & 0 & 0,105 \\
\hline $\begin{array}{r}\text { European Integration and } \\
\text { Economic Methodology and } \\
\text { Research: Questions and } \\
\text { Speculations }\end{array}$ & Rothschild,-Kurt & $\begin{array}{r}\text { Atlantic-Economic- } \\
\text { Journal; } 27(3), \\
\text { September 1999, pages } \\
243-53 .\end{array}$ & 1999 & Rothschild & Kurt & WIFO & 0 & 1 & 1 & 0 & \\
\hline To Push and to Be Pushed & $\begin{array}{r}\text { Rothschild,- } \\
\text { Kurt-W. }\end{array}$ & $\begin{array}{r}\text { American-Economist; } \\
43(1), \text { Spring 1999, } \\
\text { pages 1-8. }\end{array}$ & 1999 & Rothschild & Kurt & WIFO & 0 & 0,5 & 1 & 0 & \\
\hline $\begin{array}{r}\text { laximising, Satisficing, Weights } \\
\text { and the Evaluation of } \\
\text { Macroeconomic Comparisons }\end{array}$ & $\begin{array}{r}\text { Rothschild,-- } \\
\text { Kurt-W. }\end{array}$ & $\begin{array}{r}\text { International-Review-of- } \\
\text { Applied-Economics; } \\
\text { 13(2), May 1999, pages } \\
207-17 .\end{array}$ & 1999 & Rothschild & Kurt & WIFO & 0 & 1 & 1 & 0 & \\
\hline
\end{tabular}




\begin{tabular}{|c|c|c|c|c|c|c|c|c|c|c|c|}
\hline $\begin{array}{r}\text { Spruchliches und } \\
\text { Widerspruchliches: } \\
\text { Ammerkungen zur kognitiven } \\
\text { Jissonanz in der Okonomie. (A } \\
\text { te on Cognitive Dissonances in } \\
\text { Economics. With English } \\
\text { summary.) }\end{array}$ & $\begin{array}{l}\text { Rothschild,-- } \\
\text { Kurt-W. }\end{array}$ & $\begin{array}{r}\text { Konjunkturpolitik; 44(2), } \\
\text { 1998, pages 101-13. }\end{array}$ & 1998 & Rothschild & Kurt & WIFO & 0 & 1 & 1 & 0 & \\
\hline $\begin{array}{r}\text { asiseinkommen und Alternative } \\
\text { Motivierungen }\end{array}$ & $\begin{array}{l}\text { Rothschild,-- } \\
\text { Kurt-W. }\end{array}$ & $\begin{array}{r}\text { Jahrbucher-fur- } \\
\text { Nationalokonomie-und- } \\
\text { Statistik; 216(3), May } \\
\text { 1997, pages 361-67. }\end{array}$ & 1997 & Rothschild & Kurt & WIFO & 0,21 & 0,5 & 1 & 0,105 & \\
\hline $\begin{array}{r}\text { ynes' General Theory: A Look } \\
\text { at the Criss-Cross of Reviews }\end{array}$ & Rothschild,-Kurt & $\begin{array}{r}\text { Journal-of-Post- } \\
\text { Keynesian-Economics; } \\
\text { 18(4), Summer 1996, } \\
\text { pages 533-45. }\end{array}$ & 1996 & Rothschild & Kurt & WIFO & 0,29 & 1 & 1 & 0,29 & \\
\hline I Search of a European Identity & $\begin{array}{r}\text { Rothschild,-- } \\
\text { Kurt-W. }\end{array}$ & $\begin{array}{r}\text { Kyklos; } 48(2), 1995 \\
\text { pages } 273-77 .\end{array}$ & 1995 & Rothschild & Kurt & WIFO & 0,37 & 0,5 & 1 & 0,185 & \\
\hline $\begin{array}{l}\text { The Political Economy of } \\
\text { Reciprocity: A Comment }\end{array}$ & $\begin{array}{l}\text { Rothschild,-- } \\
\text { Kurt-W. }\end{array}$ & $\begin{array}{r}\text { Banca-Nazionale-del- } \\
\text { Lavoro-Quarterly- } \\
\text { Review; 48(192), March } \\
\text { 1995, pages 109-13. }\end{array}$ & 1995 & Rothschild & Kurt & WIFO & 0 & 0,5 & 1 & 0 & \\
\hline Josef Steindl: $1912-1993$ & $\begin{array}{l}\text { Rothschild,-- } \\
\text { Kurt-W. }\end{array}$ & $\begin{array}{r}\text { Economic-Journal; } \\
\text { 104(422), January } \\
1994, \text { pages 131-37. }\end{array}$ & 1994 & Rothschild & Kurt & WIFO & 1,21 & 0,5 & 1 & 0,605 & \\
\hline New World Economic Order & $\begin{array}{l}\text { Rothschild,-- } \\
\text { Kurt-W. }\end{array}$ & $\begin{array}{r}\text { International-Review-of- } \\
\text { Applied-Economics; } \\
\text { 7(3), 1993, pages 243- } \\
52 .\end{array}$ & 1993 & Rothschild & Kurt & WIFO & 0 & 0,5 & 1 & 0 & \\
\hline $\begin{array}{r}\text { Like a Lehrstuck by Brecht: } \\
\text { Notes on the German } \\
\text { Reunification Drama }\end{array}$ & $\begin{array}{l}\text { Rothschild,-- } \\
\text { Kurt-W. }\end{array}$ & $\begin{array}{r}\text { Cambridge-Journal-of- } \\
\text { Economics; } 17(3), \\
\text { September 1993, pages } \\
259-66 .\end{array}$ & 1993 & Rothschild & Kurt & WIFO & 0,71 & 0,5 & 1 & 0,355 & \\
\hline $\begin{array}{r}\text { The End of History? } \\
\text { Afterthoughts } \\
\end{array}$ & $\begin{array}{l}\text { Rothschild,-- } \\
\text { Kurt-W. }\end{array}$ & $\begin{array}{r}\text { Kyklos; 45(4), 1992, } \\
\text { pages 555-59. }\end{array}$ & 1992 & Rothschild & Kurt & WIFO & 0,37 & 0,5 & 1 & 0,185 & \\
\hline $\begin{array}{r}\text { Jote on Insiders, Outsiders and } \\
\text { the Two-Thirds-Society }\end{array}$ & $\begin{array}{l}\text { Rothschild,-- } \\
\text { Kurt-W. }\end{array}$ & $\begin{array}{r}\text { Kyklos; 44(2), 1991, } \\
\text { pages 233-39. }\end{array}$ & 1991 & Rothschild & Kurt & WIFO & 0,37 & 0,5 & 1 & 0,185 & \\
\hline $\begin{array}{r}\text { Glimses of a Non-linear } \\
\text { Biography }\end{array}$ & $\begin{array}{l}\text { Rothschild,-- } \\
\text { Kurt-W. }\end{array}$ & $\begin{array}{r}\text { Banca-Nazionale-del- } \\
\text { Lavoro-Quarterly- } \\
\text { Review; 0(176), March } \\
\text { 1991, pages 3-13. }\end{array}$ & 1991 & Rothschild & Kurt & WIFO & 0 & 0,5 & 1 & 0 & K. Rothschild: \\
\hline
\end{tabular}




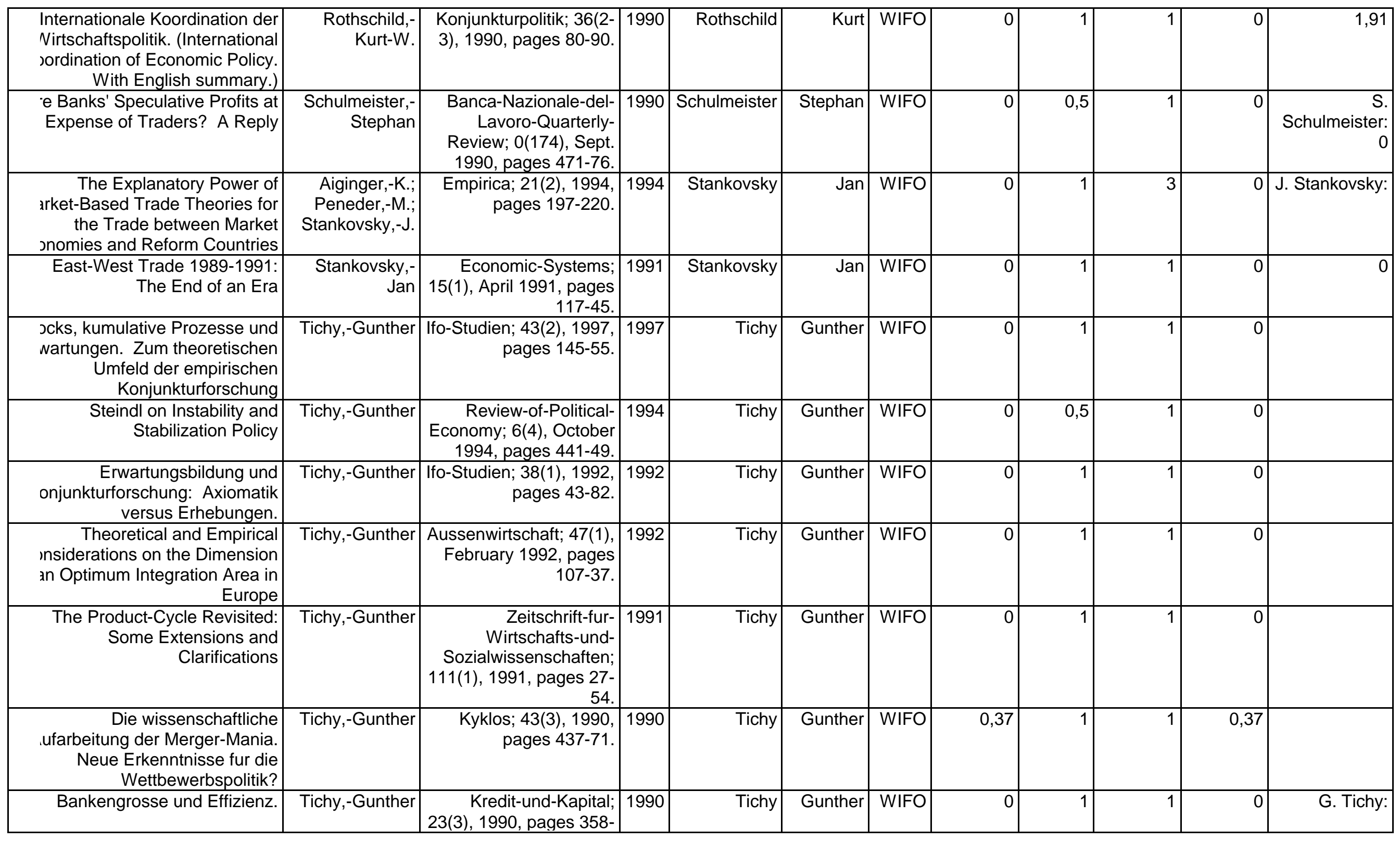




\begin{tabular}{|c|c|c|c|c|c|c|c|c|c|c|c|}
\hline & & 88. & & & & & & & & & \\
\hline $\begin{array}{r}\text { Die Praxis der } \\
\text { Konjunkturprognose des } \\
\text { Sachverstandigenrates: } 1 . \\
\text { Korreferat }\end{array}$ & Tichy,-Gunther & $\begin{array}{l}\text { Ifo-Studien; 36(2-3), } \\
\text { 1990, pages 119-26. }\end{array}$ & 1990 & Tichy & Gunther & WIFO & 0 & 0,5 & 1 & 0 & 0,37 \\
\hline ine Wahrungsunion in Europa. & $\begin{array}{r}\text { Helmenstein,- } \\
\text { Christian; Url,- } \\
\text { Thomas } \\
\end{array}$ & $\begin{array}{r}\text { Konjunkturpolitik; 39(4), } \\
1993 \text {, pages } 251-63 .\end{array}$ & 1993 & Url & Thomas & WIFO & 0 & 1 & 2 & 0 & T. Url: \\
\hline $\begin{array}{l}\text { The Nature of Austrian } \\
\text { Macroeconomic Time Series }\end{array}$ & $\begin{array}{r}\text { Url,-Thomas; } \\
\text { Wehinger,-Gert }\end{array}$ & $\begin{array}{r}\text { Empirica; } 17(2), 1990, \\
\text { pages } 131-54 .\end{array}$ & 1990 & $\mathrm{Url}$ & Thomas & WIFO & 0 & 1 & 2 & 0 & 0 \\
\hline
\end{tabular}

Average: 0,224885 
WU-Wien

\begin{tabular}{|c|c|c|c|c|c|c|c|c|c|c|c|c|}
\hline & Author(s) & Journal & Year & & & & & $\begin{array}{l}\text { Impact } \\
\text { Factor }\end{array}$ & $\begin{array}{c}\text { Seiten- } \\
\text { korr. }\end{array}$ & Autoren & Impact & personal Impact \\
\hline $\begin{array}{l}\text { Specialization, Product Differentiation } \\
\text { and Ownership Structure in Personal } \\
\text { Social Services: The Case of Nursery } \\
\text { Schools }\end{array}$ & $\begin{array}{l}\text { Badelt,-Christoph; } \\
\text { Weiss,-Peter }\end{array}$ & $\begin{array}{l}\text { Kyklos; 43(1), 1990, } \\
\text { pp. 69-89. }\end{array}$ & 1990 & Badelt & Christoph & $\begin{array}{l}\text { WU- } \\
\text { Wien }\end{array}$ & & 0.37 & 1 & 2 & 0.246666667 & C. Badelt: 0.2466667 \\
\hline $\begin{array}{l}\text { The Measurement of Foreign Direct } \\
\text { Investment: A Critical Review }\end{array}$ & Bellak,-Christian & \begin{tabular}{l|} 
International-Trade- \\
Journal; $12(2)$, Summer \\
1998, pp. 227-57.
\end{tabular} & 1998 & Bellak & Christian & $\begin{array}{l}\text { WU- } \\
\text { Wien }\end{array}$ & & 0 & 1 & 1 & 0 & \\
\hline $\begin{array}{l}\text { How Important Is Foreign Direct } \\
\text { Investment? }\end{array}$ & $\begin{array}{l}\text { Cantwell,-John; } \\
\text { Bellak,-Christian }\end{array}$ & $\begin{array}{l}\text { Oxford-Bulletin-of- } \\
\text { Economics-and-Statistics; } \\
\text { 60(1), February 1998, } \\
\text { pp. 99-106. }\end{array}$ & 1998 & Bellak & Christian & $\begin{array}{l}\text { WU- } \\
\text { Wien }\end{array}$ & & 0.76 & 0.5 & 2 & 0.253333333 & \\
\hline $\begin{array}{l}\text { Foreign Direct Investment--How Much } \\
\text { Is It Worth? Comment }\end{array}$ & $\begin{array}{l}\text { Bellak,-Christian; } \\
\text { Cantwell,-John }\end{array}$ & \begin{tabular}{l|} 
Transnational- \\
Corporations; 5(1), April \\
1996, pp. 85-97.
\end{tabular} & 1996 & Bellak & Christian & $\begin{array}{l}\text { WU- } \\
\text { Wien }\end{array}$ & & 0 & 1 & 2 & 0 & \\
\hline Reeling in the Transnationals & Bellak,-Christian & $\begin{array}{l}\text { New -Economy; 4(1), } \\
\text { Spring 1997, pp. 17-21. }\end{array}$ & 1997 & Bellak & Christian & $\begin{array}{l}\text { WU- } \\
\text { Wien }\end{array}$ & & 0 & 0.5 & 1 & 0 & \\
\hline $\begin{array}{l}\text { Austrian Manufacturing MNEs: Long- } \\
\text { Term Perspectives }\end{array}$ & Bellak,-Christian & $\begin{array}{l}\text { Business-History; } 39(1), \\
\text { January } 1997, \text { pp. } 47-71 .\end{array}$ & 1997 & Bellak & Christian & $\begin{array}{l}\text { WU- } \\
\text { Wien }\end{array}$ & & 0 & 1 & 1 & 0 & \\
\hline International Capital Mobility--A Note & Bellak,-Christian & $\begin{array}{l}\text { Journal-of-International- } \\
\text { Money-and-Finance; } \\
\text { 15(5), October 1996, } \\
\text { pp. 825-28. }\end{array}$ & 1996 & Bellak & Christian & $\begin{array}{l}\text { WU- } \\
\text { Wien }\end{array}$ & & 0.49 & 0.5 & 1 & 0.245 & \\
\hline $\begin{array}{l}\text { Foreign Direct Investments Bias the } \\
\text { Net External Asset Position }\end{array}$ & $\begin{array}{l}\text { Bellak,-Christian- } \\
\text { J. }\end{array}$ & $\begin{array}{l}\text { Applied-Economics- } \\
\text { Letters; 2(4), April 1995, } \\
\text { pp. 83-85. }\end{array}$ & 1995 & Bellak & Christian & $\begin{array}{l}\text { WU- } \\
\text { Wien }\end{array}$ & & 0 & 0.5 & 1 & 0 & $\begin{array}{l}\text { C. Bellak: } \\
0.498333333\end{array}$ \\
\hline $\begin{array}{l}\text { How Reliable Are Estimations of East- } \\
\text { West Trade Potentials Based on } \\
\text { Cross-Section Gravity Analyses? }\end{array}$ & $\begin{array}{l}\text { Breuss,-Fritz; } \\
\text { Egger,-Peter }\end{array}$ & $\begin{array}{l}\text { Empirica; 26(2), 1999, } \\
\text { pp. 81-94. }\end{array}$ & 1999 & Breuss & Fritz & $\begin{array}{l}\text { WU- } \\
\text { Wien }\end{array}$ & WIFO & 0 & 1 & 2 & 0 & \\
\hline $\begin{array}{l}\text { Biomass Energy Use to Reduce } \\
\text { Climate Change: A General } \\
\text { Equilibrium Analysis for Austria }\end{array}$ & $\begin{array}{l}\text { Breuss,-Fritz; } \\
\text { Steininger,-Karl }\end{array}$ & $\begin{array}{l}\text { Journal-of-Policy- } \\
\text { Modeling; 20(4), August } \\
\text { 1998, pp. 513-35. }\end{array}$ & 1998 & Breuss & Fritz & $\begin{array}{l}\text { WU- } \\
\text { Wien }\end{array}$ & WIFO & 0.12 & 1 & 2 & 0.08 & \\
\hline $\begin{array}{l}\text { A General Equilibrium Evaluation of } \\
\text { Trade and Industrial Policy Changes } \\
\text { in Austria and Hungary }\end{array}$ & $\begin{array}{l}\text { Breuss,-Fritz; } \\
\text { Tesche,--Jean }\end{array}$ & $\begin{array}{l}\text { Weltwirtschaftliches- } \\
\text { Archiv; 130(3), 1994, pp. } \\
\text { 534-52. }\end{array}$ & 1994 & Breuss & Fritz & $\begin{array}{l}\text { WU- } \\
\text { Wien }\end{array}$ & WIFO & 0.45 & 1 & 2 & 0.3 & \\
\hline $\begin{array}{l}\text { Hungary in Transition: A Computable } \\
\text { General Equilibrium Model } \\
\text { Comparison with Austria }\end{array}$ & $\begin{array}{l}\text { Breuss,-Fritz; } \\
\text { Tesche,-Jean }\end{array}$ & $\begin{array}{l}\text { Journal-of-Policy- } \\
\text { Modeling; 15(5-6), Oct.- } \\
\text { Dec. 1993, pp. 581-623. }\end{array}$ & 1993 & Breuss & Fritz & $\begin{array}{l}\text { WU- } \\
\text { Wien }\end{array}$ & WIFO & 0.12 & 1 & 2 & 0.08 & \\
\hline $\begin{array}{l}\text { A CGE Model for Austria: Some } \\
\text { Implications of Trade Liberalization }\end{array}$ & $\begin{array}{l}\text { Breuss,-Fritz; } \\
\text { Tesche,-Jean }\end{array}$ & $\begin{array}{l}\text { Empirica; 18(2), 1991, } \\
\text { pp. 135-65. }\end{array}$ & 1991 & Breuss & Fritz & $\begin{array}{l}\text { WU- } \\
\text { Wien }\end{array}$ & WIFO & 0 & 1 & 2 & 0 & \\
\hline $\begin{array}{l}\text { Internationaler Handel mit } \\
\text { Dienstleistungen--theoretische } \\
\text { Ansatze. }\end{array}$ & Breuss,-Fritz & $\begin{array}{l}\text { Aussenwirtschaft; 45(1), } \\
\text { April 1990, pp. 105-30. }\end{array}$ & 1990 & Breuss & Fritz & $\begin{array}{l}\text { WU- } \\
\text { Wien }\end{array}$ & WIFO & 0 & 1 & 1 & 0 & F. Breuss: 0.46 \\
\hline $\begin{array}{l}\text { Wage Differentials between Skilled } \\
\text { and Unskilled Workers }\end{array}$ & Heise,-Arne & $\begin{array}{l}\text { Jahrbucher-fur- } \\
\text { Nationalokonomie-und- } \\
\text { Statistik; 216(3), May } \\
\text { 1997, pp. 368-72. }\end{array}$ & 1997 & Heise & Arne & $\begin{array}{l}\text { WU- } \\
\text { Wien }\end{array}$ & & 0.21 & 0.5 & 1 & 0.105 & \\
\hline
\end{tabular}


WU-Wien

\begin{tabular}{|c|c|c|c|c|c|c|c|c|c|c|c|}
\hline & Author(s) & Journal & Year & & & & $\begin{array}{l}\text { Impact } \\
\text { Factor } \\
\end{array}$ & $\begin{array}{c}\text { Seiten- } \\
\text { korr. }\end{array}$ & Autoren & Impact & personal Impact \\
\hline $\begin{array}{l}\text { A Different Transatlantic View: The } \\
\text { American Job Machine }\end{array}$ & Heise,-Arne & $\begin{array}{l}\text { Challenge; 40(3), May- } \\
\text { June 1997, pp. 50-56. }\end{array}$ & 1997 & Heise & Arne & $\begin{array}{l}\text { WU- } \\
\text { Wien }\end{array}$ & 0 & 0.5 & 1 & 0 & \\
\hline Geldpolitik im Disput. & Heise,-Arne & $\begin{array}{l}\text { Konjunkturpolitik; 38(4), } \\
\text { 1992, pp. 175-94. }\end{array}$ & 1992 & Heise & Arne & $\begin{array}{l}\text { WU- } \\
\text { Wien } \\
\end{array}$ & 0 & 1 & 1 & 0 & \\
\hline $\begin{array}{l}\text { Money and Credit or a Never Ending } \\
\text { Story }\end{array}$ & Heise,-Arne & $\begin{array}{l}\text { Jahrbucher-fur- } \\
\text { Nationalokonomie-und- } \\
\text { Statistik; 210(3-4), Sept. } \\
\text { 1992, pp. 266-78. }\end{array}$ & 1992 & Heise & Arne & $\begin{array}{l}\text { WU- } \\
\text { Wien }\end{array}$ & 0.21 & 1 & 1 & 0.21 & \\
\hline $\begin{array}{l}\text { Commercial Banks in Macroeconomic } \\
\text { Theory }\end{array}$ & Heise,-Arne & $\begin{array}{l}\text { Journal-of-Post- } \\
\text { Keynesian-Economics; } \\
\text { 14(3), Spring 1992, } \\
\text { pp. 285-96. }\end{array}$ & 1992 & Heise & Arne & $\begin{array}{l}\text { WU- } \\
\text { Wien }\end{array}$ & 0.29 & 1 & 1 & 0.29 & \\
\hline $\begin{array}{l}\text { Einige Anmerkungen zur } \\
\text { Investitionstheorie-Debatte. }\end{array}$ & Heise,-Arne & $\begin{array}{l}\text { Jahrbucher-fur- } \\
\text { Nationalokonomie-und- } \\
\text { Statistik; 207(5), Sept. } \\
\text { 1990, pp. 432-47. }\end{array}$ & 1990 & Heise & Arne & $\begin{array}{l}\text { WU- } \\
\text { Wien }\end{array}$ & 0.21 & 1 & 1 & 0.21 & A. Heise: 0.815 \\
\hline $\begin{array}{l}\text { Equilibrium Methodology as Seen } \\
\text { from a Hayekian Perspective }\end{array}$ & $\begin{array}{l}\text { Klausinger,- } \\
\text { Hansjoerg }\end{array}$ & \begin{tabular}{|l|} 
Journal-of-the-History-of- \\
Economic-Thought; $12(1)$, \\
Spring 1990, pp. $61-75$.
\end{tabular} & 1990 & Klausinger & Hansjoerg & $\begin{array}{l}\text { WU- } \\
\text { Wien }\end{array}$ & 0 & 1 & 1 & 0 & \\
\hline $\begin{array}{l}\text { A Consistent Formulation of the } \\
\text { Leontief Pollution Model }\end{array}$ & $\begin{array}{l}\text { Luptacik,-Mikulas; } \\
\text { Bohm,-Bernhard }\end{array}$ & \begin{tabular}{|l|} 
Economic-Systems - \\
Research; 11(3), \\
September 1999, pp. 263- \\
75. \\
\end{tabular} & 1999 & Luptacik & Mikulas & $\begin{array}{l}\text { WU- } \\
\text { Wien }\end{array}$ & 0 & 1 & 2 & 0 & \\
\hline $\begin{array}{l}\text { The Accomplishment of the Maastricht } \\
\text { Criteria with Respect to Initial Debt }\end{array}$ & $\begin{array}{l}\text { Prskawetz,-A.; } \\
\text { Feichtinger,-G.; } \\
\text { Luptacik,-M. }\end{array}$ & $\begin{array}{l}\text { Journal-of-Economics- } \\
\text { (Zeitschrift-fur- } \\
\text { Nationalokonomie); 68(2), } \\
\text { 1998, pp. 93-110. }\end{array}$ & 1998 & Luptacik & Mikulas & $\begin{array}{l}\text { WU- } \\
\text { Wien }\end{array}$ & 0.18 & 1 & 3 & 0.09 & \\
\hline $\begin{array}{l}\text { Reconsideration of Non-negative } \\
\text { Solutions for the Augmented Leontief } \\
\text { Model }\end{array}$ & $\begin{array}{l}\text { Luptacik,-Mikulas; } \\
\text { Bohm,-Bernhard }\end{array}$ & $\begin{array}{l}\text { Economic-Systems - } \\
\text { Research; 6(2), 1994, } \\
\text { pp. 167-70. }\end{array}$ & 1994 & Luptacik & Mikulas & $\begin{array}{l}\text { WU- } \\
\text { Wien }\end{array}$ & 0 & 0.5 & 2 & 0 & \\
\hline $\begin{array}{l}\text { Environmental Constraints and Choice } \\
\text { of Technology: A Dynamic Activity } \\
\text { Analysis }\end{array}$ & $\begin{array}{l}\text { Hartl,-Richard-F.; } \\
\text { Luptacik,-Mikulas }\end{array}$ & $\begin{array}{l}\text { Czechoslovak-Journal- } \\
\text { for-Operations-Research; } \\
1(2), 1992, \text { pp. 107-25. } \\
\end{array}$ & 1992 & Luptacik & Mikulas & $\begin{array}{l}\text { WU- } \\
\text { Wien }\end{array}$ & 0 & 1 & 2 & 0 & \\
\hline $\begin{array}{l}\text { Optimal Dynamic Environmental } \\
\text { Policies of a Profit Maximizing Firm }\end{array}$ & $\begin{array}{l}\text { Kort,-P.-M.; van- } \\
\text { Loon,-P.--J.-J.-M.; } \\
\text { Luptacik,-M. }\end{array}$ & $\begin{array}{l}\text { Journal-of-Economics- } \\
\text { (Zeitschrift-fur- } \\
\text { Nationalokonomie); 54(3), } \\
\text { 1991, pp. 195-225. }\end{array}$ & 1991 & Luptacik & Mikulas & $\begin{array}{l}\text { WU- } \\
\text { Wien }\end{array}$ & 0.18 & 1 & 3 & 0.09 & M. Luptacik: 0.18 \\
\hline $\begin{array}{l}\text { Effizienz, Skalen- und Verbundvorteile } \\
\text { deutscher Versicherer. }\end{array}$ & \begin{tabular}{|l|} 
Mahlberg,-- \\
Bernhard \\
\end{tabular} & $\begin{array}{l}\text { Ifo-Studien; 45(3), 1999, } \\
\text { pp. 335-69. }\end{array}$ & 1999 & Mahlberg & Bernhard & \begin{tabular}{|l} 
WU- \\
Wien
\end{tabular} & 0 & 1 & 1 & 0 & B. Mahlberg: 0 \\
\hline $\begin{array}{l}\text { The Importance of Crowding-Out for } \\
\text { the Effectiveness of Fiscal Policy in } \\
\text { Austria }\end{array}$ & $\begin{array}{l}\text { Munduch,-- } \\
\text { Gerhard }\end{array}$ & $\begin{array}{l}\text { Empirica; 18(1), 1991, } \\
\text { pp. 65-89. }\end{array}$ & 1991 & Munduch & Gerhard & $\begin{array}{l}\text { WU- } \\
\text { Wien }\end{array}$ & 0 & 1 & 1 & 0 & G. Munduch: 0 \\
\hline $\begin{array}{l}\text { Privatization in Austria: Causes and } \\
\text { Consequences }\end{array}$ & Nowotny,-Ewald & $\begin{array}{l}\text { Annals-of-Public-and- } \\
\text { Cooperative-Economics; } \\
\text { 67(3), Sept. 1996, pp. } \\
\text { 387-401. }\end{array}$ & 1996 & Nowotny & Ewald & $\begin{array}{l}\text { WU- } \\
\text { Wien }\end{array}$ & 0 & 1 & 1 & 0 & E. Novotny: 0 \\
\hline
\end{tabular}


WU-Wien

\begin{tabular}{|c|c|c|c|c|c|c|c|c|c|c|c|}
\hline & Author(s) & Journal & Year & & & & \begin{tabular}{|l} 
Impact \\
Factor
\end{tabular} & $\begin{array}{c}\text { Seiten- } \\
\text { korr. }\end{array}$ & Autoren & Impact & personal Impact \\
\hline $\begin{array}{l}\text { Sector-Specific Regulation from a } \\
\text { Public Choice Perspective with } \\
\text { Regard to the Supply of Services of } \\
\text { Public Interest }\end{array}$ & $\begin{array}{l}\text { Obermann,- } \\
\text { Gabriel }\end{array}$ & $\begin{array}{l}\text { Annals-of-Public-and- } \\
\text { Cooperative-Economics; } \\
\text { 70(2), June 1999, } \\
\text { pp. 195-211. } \\
\end{array}$ & 1999 & Obermann & Gabriel & $\begin{array}{l}\text { WU- } \\
\text { Wien }\end{array}$ & 0 & 1 & 1 & 0 & G. Obermann: 0 \\
\hline Quality with Saturation Goods & Pichler,-Eva & $\begin{array}{l}\text { Zeitschrift-fur-Wirtschafts- } \\
\text {-und- } \\
\text { Sozialwissenschaften; } \\
117(4), 1997 \text {, pp. 545-65. }\end{array}$ & 1997 & Pichler & Eva & $\begin{array}{l}\text { WU- } \\
\text { Wien }\end{array}$ & 0 & 1 & 1 & 0 & \\
\hline $\begin{array}{l}\text { Wage Parity and Patterns of } \\
\text { Unionization }\end{array}$ & $\begin{array}{l}\text { Winkler,-G.- } \\
\text { Michael; Pichler,-- } \\
\text { Eva }\end{array}$ & $\begin{array}{l}\text { Review -of-Political- } \\
\text { Economy; 10(1), January } \\
\text { 1998, pp. 57-71. }\end{array}$ & 1998 & Pichler & Eva & $\begin{array}{l}\text { WU- } \\
\text { Wien }\end{array}$ & 0 & 1 & 2 & 0 & \\
\hline $\begin{array}{l}\text { Efficiency Wages and Union Wage } \\
\text { Bargaining }\end{array}$ & Pichler,-Eva & $\begin{array}{l}\text { Jahrbucher-fur- } \\
\text { Nationalokonomie-und- } \\
\text { Statistik; 212(1-2), July } \\
\text { 1993, pp. 140-50. } \\
\end{array}$ & 1993 & Pichler & Eva & $\begin{array}{l}\text { WU- } \\
\text { Wien }\end{array}$ & 0.21 & 1 & 1 & 0.21 & \\
\hline $\begin{array}{l}\text { Cost-Sharing of General and Specific } \\
\text { Training with Depreciation of Human } \\
\text { Capital }\end{array}$ & Pichler,-Eva & $\begin{array}{l}\text { Economics-of-Education- } \\
\text { Review; 12(2), June } \\
\text { 1993, pp. 117-24. } \\
\end{array}$ & 1993 & Pichler & Eva & $\begin{array}{l}\text { WU- } \\
\text { Wien }\end{array}$ & 0 & 0.5 & 1 & 0 & \\
\hline $\begin{array}{l}\text { Cost of Negotiations and the Structure } \\
\text { of Bargaining--A Note }\end{array}$ & Pichler,-Eva & $\begin{array}{l}\text { Zeitschrift-fur-Wirtschafts- } \\
\text { und- } \\
\text { Sozialwissenschaften; } \\
\text { 113(1), 1993, pp. 115-21. }\end{array}$ & 1993 & Pichler & Eva & $\begin{array}{l}\text { WU- } \\
\text { Wien }\end{array}$ & 0 & 0.5 & 1 & 0 & \\
\hline Union Wage Bargaining and Status & Pichler,-Eva & $\begin{array}{l}\text { Jahrbucher-fur- } \\
\text { Nationalokonomie-und- } \\
\text { Statistik; 210(3-4), Sept. } \\
\text { 1992, pp. 358-62. } \\
\end{array}$ & 1992 & Pichler & Eva & $\begin{array}{l}\text { WU- } \\
\text { Wien }\end{array}$ & 0.21 & 0.5 & 1 & 0.105 & \\
\hline $\begin{array}{l}\text { Investment in General and Job- } \\
\text { Specific Training with Occupational } \\
\text { Mobility }\end{array}$ & Pichler,-Eva & $\begin{array}{l}\text { Jahrbucher-fur- } \\
\text { Nationalokonomie-und- } \\
\text { Statistik; 210(1-2), July } \\
\text { 1992, pp. 35-46. }\end{array}$ & 1992 & Pichler & Eva & $\begin{array}{l}\text { WU- } \\
\text { Wien }\end{array}$ & 0.21 & 1 & 1 & 0.21 & \\
\hline $\begin{array}{l}\text { A Note on Human Capital } \\
\text { Accumulation and Unemployment }\end{array}$ & Pichler,-Eva & $\begin{array}{l}\text { Jahrbucher-fur- } \\
\text { Nationalokonomie-und- } \\
\text { Statistik; 208(6), Nov. } \\
\text { 1991, pp. 561-74. } \\
\end{array}$ & 1991 & Pichler & Eva & $\begin{array}{l}\text { WU- } \\
\text { Wien }\end{array}$ & 0.21 & 1 & 1 & 0.21 & E. Pichler: 0.735 \\
\hline $\begin{array}{l}\text { Sense of Community: A Fundamental } \\
\text { Concept of Institutional Economics }\end{array}$ & $\begin{array}{l}\text { Pirker,-Reinhard; } \\
\text { Rauchenschwand } \\
\text { ner,--Hermann }\end{array}$ & $\begin{array}{l}\text { Journal-of-Institutional- } \\
\text { and-Theoretical- } \\
\text { Economics; } 154(2), \text { June } \\
\text { 1998, pp. 406-21. } \\
\end{array}$ & 1998 & Pirker, & Reinhard & $\begin{array}{l}\text { WU- } \\
\text { Wien }\end{array}$ & 0.29 & 1 & 2 & 0.193333333 & R. Pirker: 0.19333 \\
\hline $\begin{array}{l}\text { Sense of Community: A Fundamental } \\
\text { Concept of Institutional Economics }\end{array}$ & $\begin{array}{l}\text { Pirker,-Reinhard; } \\
\text { Rauchenschwand } \\
\text { ner,--Hermann }\end{array}$ & $\begin{array}{l}\text { Journal-of-Institutional- } \\
\text { and-Theoretical- } \\
\text { Economics; 154(2), June } \\
\text { 1998, pp. 406-21. }\end{array}$ & 1998 & $\begin{array}{l}\text { Rauchenschw } \\
\text { andtner }\end{array}$ & Hermann & $\begin{array}{l}\text { WU- } \\
\text { Wien }\end{array}$ & 0.29 & 1 & 2 & 0.193333333 & $\begin{array}{l}\text { Rauchenschwandtner: } \\
0.19333\end{array}$ \\
\hline $\begin{array}{l}\text { Wage Setting and Hysteresis in } \\
\text { Unemployment }\end{array}$ & Stiassny,-Alfred & $\begin{array}{l}\text { Empirica; } 25(1), 1998, \\
\text { pp. } 79-107 .\end{array}$ & 1998 & Stiassny & Alfred & $\begin{array}{l}\text { WU- } \\
\text { Wien }\end{array}$ & 0 & 1 & 1 & 0 & \\
\hline $\begin{array}{l}\text { Tax and Spend, or Spend and Tax? } \\
\text { An International Study }\end{array}$ & $\begin{array}{l}\text { Koren,-Stephan; } \\
\text { Stiassny,-Alfred }\end{array}$ & $\begin{array}{l}\text { Journal-of-Policy- } \\
\text { Modeling; 20(2), April } \\
\text { 1998, pp. 163-91. }\end{array}$ & 1998 & Stiassny & Alfred & $\begin{array}{l}\text { WU- } \\
\text { Wien }\end{array}$ & 0.12 & 1 & 2 & 0.08 & \\
\hline
\end{tabular}


WU-Wien

\begin{tabular}{|c|c|c|c|c|c|c|c|c|c|c|c|}
\hline & Author(s) & Journal & Year & & & & $\begin{array}{l}\text { Impact } \\
\text { Factor }\end{array}$ & $\begin{array}{c}\text { Seiten- } \\
\text { korr. }\end{array}$ & Autoren & Impact & personal Impact \\
\hline $\begin{array}{l}\text { A Spectral Decomposition for } \\
\text { Structural VAR Models }\end{array}$ & Stiassny,-Alfred & $\begin{array}{l}\text { Empirical-Economics; } \\
21(4), 1996, \text { pp. } 535-55 .\end{array}$ & 1996 & \begin{tabular}{|l|} 
Stiassny \\
\end{tabular} & Alfred & $\begin{array}{l}\text { WU- } \\
\text { Wien }\end{array}$ & 0 & 1 & 1 & 0 & \\
\hline $\begin{array}{l}\text { Tax and Spend or Spend and Tax? } \\
\text { An Empirical Investigation for Austria }\end{array}$ & $\begin{array}{l}\text { Koren,-Stephan; } \\
\text { Stiassny,-Alfred }\end{array}$ & $\begin{array}{l}\text { Empirica; 22(2), } 1995, \\
\text { pp. 127-49. }\end{array}$ & 1995 & Stiassny & Alfred & $\begin{array}{l}\text { WU- } \\
\text { Wien }\end{array}$ & 0 & 1 & 2 & 0 & \\
\hline $\begin{array}{l}\text { Long-term Impacts of Import Prices on } \\
\text { Domestic Prices and Wages: An } \\
\text { Empirical Investigation for Austria }\end{array}$ & Stiassny,-Alfred & $\begin{array}{l}\text { Empirica; } 17(1), 1990, \\
\text { pp. 15-40. }\end{array}$ & 1990 & Stiassny & Alfred & $\begin{array}{l}\text { WU- } \\
\text { Wien }\end{array}$ & 0 & 1 & 1 & 0 & A. Stiassny: 0.08 \\
\hline $\begin{array}{l}\text { The Index of Sustainable Economic } \\
\text { Welfare (ISEW) as an Alternative to } \\
\text { GDP in Measuring Economic Welfare. } \\
\text { The Results of the Austrian ISEW ... }\end{array}$ & $\begin{array}{l}\text { Stockhammer,- } \\
\text { Engelbert et-al. }\end{array}$ & $\begin{array}{l}\text { Ecological-Economics; } \\
21(1) \text {, April 1997, pp. 19- } \\
34 .\end{array}$ & 1997 & Stockhammer & Engelbert & $\begin{array}{l}\text { WU- } \\
\text { Wien }\end{array}$ & 0.64 & 1 & 3 & 0.32 & E. Stockhammer: 0.32 \\
\hline $\begin{array}{l}\text { The Changing Pattern of Regional } \\
\text { Convergence in Europe }\end{array}$ & Tondl,-Gabriele & \begin{tabular}{l|} 
Jahrbuch-fur- \\
Regionalwiss./Review -of- \\
Regional-Research; \\
19(1), 1999, pp. 1-33.
\end{tabular} & 1999 & Tondl & Gabriele & $\begin{array}{l}\text { WU- } \\
\text { Wien }\end{array}$ & 0 & 1 & 1 & 0 & G. Tondl: 0 \\
\hline $\begin{array}{l}\text { Governance, Economic Restructuring, } \\
\text { and International Competitiveness }\end{array}$ & \begin{tabular}{|l|} 
Traxler,-Franz; \\
Unger,-Brigitte
\end{tabular} & $\begin{array}{l}\text { Journal-of-Economic- } \\
\text { Issues; 28(1), March } \\
\text { 1994, pp. 1-23. }\end{array}$ & 1994 & Unger & Brigitte & $\begin{array}{l}\text { WU- } \\
\text { Wien }\end{array}$ & 0.46 & 1 & 2 & 0.306666667 & B. Unger: 0.30666 \\
\hline $\begin{array}{l}\text { Separating Small and Big Fish: The } \\
\text { Case of Income Tax Evasion }\end{array}$ & $\begin{array}{l}\text { Falkinger,-Josef; } \\
\text { Walther,-Herbert }\end{array}$ & $\begin{array}{l}\text { Journal-of-Economics- } \\
\text { (Zeitschrift-fur- } \\
\text { Nationalokonomie); 54(1), } \\
\text { 1991, pp. 55-67. }\end{array}$ & 1991 & Walther & Herbert & $\begin{array}{l}\text { WU- } \\
\text { Wien }\end{array}$ & 0.18 & 1 & 2 & 0.12 & \\
\hline $\begin{array}{l}\text { Rewards versus Penalties: On a New } \\
\text { Policy Against Tax Evasion }\end{array}$ & $\begin{array}{l}\text { Falkinger,-Josef; } \\
\text { Walther,-Herbert }\end{array}$ & $\begin{array}{l}\text { Public-Finance-Quarterly; } \\
\text { 19(1), January 1991, } \\
\text { pp. 67-79. }\end{array}$ & 1991 & Walther & Herbert & $\begin{array}{l}\text { WU- } \\
\text { Wien }\end{array}$ & 0 & 1 & 2 & 0 & H. Walther: 0.12 \\
\hline $\begin{array}{l}\text { Coalition-Sensitive Voting Power in } \\
\text { the Council of Ministers: The Case of } \\
\text { Eastern Enlargement }\end{array}$ & $\begin{array}{l}\text { Winkler,-G.- } \\
\text { Michael }\end{array}$ & $\begin{array}{l}\text { Journal-of-Common- } \\
\text { Market-Studies; 36(3), } \\
\text { September 1998, pp. 391- } \\
404 .\end{array}$ & 1998 & Winkler & Michael & $\begin{array}{l}\text { WU- } \\
\text { Wien }\end{array}$ & 0 & 1 & 1 & 0 & \\
\hline $\begin{array}{l}\text { Wage Parity and Patterns of } \\
\text { Unionization }\end{array}$ & $\begin{array}{l}\text { Winkler,-G.- } \\
\text { Michael; Pichler,-- } \\
\text { Eva }\end{array}$ & \begin{tabular}{|l|} 
Review -of-Political- \\
Economy; $10(1)$, January \\
1998, pp. $57-71$.
\end{tabular} & 1998 & Winkler & Michael & $\begin{array}{l}\text { WU- } \\
\text { Wien }\end{array}$ & 0 & 1 & 2 & 0 & M. Winkler: 0 \\
\hline $\begin{array}{l}\text { Innovation, Company Co-operation } \\
\text { and the Transformation Process in } \\
\text { Eastern Europe }\end{array}$ & $\begin{array}{l}\text { Zagler,-Martin; } \\
\text { Ragacs,-Christian }\end{array}$ & \begin{tabular}{|l|} 
Zagreb-International- \\
Review -of-Economics- \\
and-Business; $1(2), 1998$, \\
pp. 65-75.
\end{tabular} & 1998 & Zagler & Martin & $\begin{array}{l}\text { WU- } \\
\text { Wien }\end{array}$ & 0 & 1 & 2 & 0 & \\
\hline $\begin{array}{l}\text { Economic Growth and Protection of } \\
\text { Emerging Markets in Eastern Europe }\end{array}$ & Zagler,-Martin & $\begin{array}{l}\text { Journal-of-Economic- } \\
\text { Development; } 23(1) \text {, June } \\
\text { 1998, pp. } 21-36 .\end{array}$ & 1998 & Zagler & Martin & \begin{tabular}{|l|} 
WU- \\
Wien
\end{tabular} & 0 & 1 & 1 & 0 & \\
\hline $\begin{array}{l}\text { The Intertemporal New-Keynesian } \\
\text { Framework--What Is Left of Keynes }\end{array}$ & Zagler,-Martin & $\begin{array}{l}\text { Ekonomska-Misao-i- } \\
\text { Praksa; 5(0), Special } \\
\text { Issue 1996, pp. 429-59. }\end{array}$ & 1996 & Zagler & Martin & \begin{tabular}{|l|} 
WU- \\
Wien
\end{tabular} & 0 & 1 & 1 & 0 & M. Zagler: 0 \\
\hline
\end{tabular}

Average: 0.088262411 


\begin{tabular}{|c|c|c|c|c|c|c|c|c|c|c|c|}
\hline Titel & Author(s) & Journal & Year & & & & \begin{tabular}{|c|} 
Impact \\
Factor
\end{tabular} & Authors & $\begin{array}{l}\text { Page- } \\
\text { corr. }\end{array}$ & Impact & personal Impact \\
\hline $\begin{array}{l}\text { Residential Energy Demand Analysis: } \\
\text { An Empirical Application ... }\end{array}$ & $\begin{array}{l}\text { Madlener,-Reinhard; } \\
\text { Alt,-Raimund }\end{array}$ & $\begin{array}{l}\text { Empirical-Economics; } \\
\text { 21(2), 1996, pp. 203-20. }\end{array}$ & 1996 & Alt & Raimund & IHS & 0 & 2 & 1 & 0 & R. Alt: 0 \\
\hline $\begin{array}{l}\text { Family Allowances as Welfare } \\
\text { Improvements }\end{array}$ & $\begin{array}{l}\text { Felderer,-Bernhard; } \\
\text { Ritzberger,-Klaus }\end{array}$ & $\begin{array}{l}\text { Journal-of-Economics- } \\
(\mathrm{ZfN}) ; 61(1), 1995 \\
\text { pp. 11-33. }\end{array}$ & 1995 & Felderer & Bernhard & IHS & 0.18 & 2 & 1 & 0.12 & \\
\hline $\begin{array}{l}\text { Does a Public Pension System } \\
\text { Reduce Savings Rates and Birth } \\
\text { Rates? }\end{array}$ & Felderer,-Bernhard & $\begin{array}{l}\text { Journal-of-Institutional- } \\
\text { and-Theoretical- } \\
\text { Economics; 148(2), 1992, } \\
\text { pp. 314-25. }\end{array}$ & 1992 & Felderer & Bernhard & U Bochum & 0.29 & 1 & 1 & 0.29 & \\
\hline $\begin{array}{l}\text { Neoclassical Growth with } \\
\text { Microfoundations }\end{array}$ & Felderer,-Bernhard & $\begin{array}{l}\text { Journal-of-Economics- } \\
(\mathrm{ZfN}) ; 51(3), 1990 \\
\text { pp. 273-85. }\end{array}$ & 1990 & Felderer & Bernhard & U Köln & 0.18 & 1 & 1 & 0.18 & B. Felderer: 0.59 \\
\hline $\begin{array}{l}\text { Neues Herausgebergremium der } \\
\text { ZWS. }\end{array}$ & $\begin{array}{l}\text { Felderer,-Bernhard } \\
\text { et-al. }\end{array}$ & $\begin{array}{l}\text { Zeitschrift-fur-Wirtschafts--- } \\
\text { und-Sozialwissenschaften }\end{array}$ & & Felderer & Bernhard & & & & & & \\
\hline $\begin{array}{l}\text { Public Investment, Congestion, and } \\
\text { Private Capital Accumulation }\end{array}$ & $\begin{array}{l}\text { Fisher, Walter H.; } \\
\text { Turnovsky, Stephen } \\
\text { J. }\end{array}$ & \begin{tabular}{|l|} 
Economic Journal; \\
$108(447), 1998$, pp. 399- \\
413.
\end{tabular} & 1998 & Fisher & Walter & IHS & 1.21 & 2 & 1 & 0.806666667 & \\
\hline $\begin{array}{l}\text { The Composition of Government } \\
\text { Expenditure and Its Consequences .. }\end{array}$ & $\begin{array}{l}\text { Fisher, Walter H; } \\
\text { Turnovsky, Stephen J }\end{array}$ & $\begin{array}{l}\text { Journal of Economic } \\
\text { Dynamics and Control; } \\
\text { 19(4), 1995, pp. 747-786. }\end{array}$ & 1995 & Fisher & Walter & Kansas S U & 0.53 & 2 & 1 & 0.353333333 & \\
\hline $\begin{array}{l}\text { An Optimizing Analysis of the Effects } \\
\text { of World Interest Disturbances on the } \\
\text { Open Economy Term Structure of } \\
\text { Interest Rates }\end{array}$ & Fisher, Walter H. & $\begin{array}{l}\text { Journal of International } \\
\text { Money and Finance; 14(1), } \\
\text { 1995, pp. 105-126. }\end{array}$ & 1995 & Fisher & Walter & Kansas S U & 0.49 & 1 & 1 & 0.49 & W. Fisher: 1.65 \\
\hline $\begin{array}{l}\text { Fiscal Policy and the Term Structure } \\
\text { of Interest Rates ... }\end{array}$ & $\begin{array}{l}\text { Fisher, Walter H; } \\
\text { Turnovsky, Stephen } \\
\text { J. }\end{array}$ & $\begin{array}{l}\text { Journal of Money Credit } \\
\text { and Banking; 24(1), 1992, } \\
\text { pp. 1-26. }\end{array}$ & 1992 & Fisher & Walter & UWA & 0 & 2 & 1 & 0 & \\
\hline $\begin{array}{l}\text { Human Capital Depletion, Human } \\
\text { Capital Formation, and Migration:... }\end{array}$ & $\begin{array}{l}\text { Stark,-O.; } \\
\text { Helmenstein,-C.; } \\
\text { Prskawetz,-A. } \\
\end{array}$ & $\begin{array}{l}\text { Economics-Letters; 60(3), } \\
\text { September 1998, pp. 363- } \\
67\end{array}$ & 1998 & Helmenstein & Christian & IHS & 0.26 & 3 & 0.5 & 0.065 & \\
\hline A Brain Gain with a Brain Drain & $\begin{array}{l}\text { Stark,-O.; } \\
\text { Helmenstein,-C.; } \\
\text { Prskawetz,-A. }\end{array}$ & $\begin{array}{l}\text { Economics-Letters; 55(2), } \\
\text { August 1997, pp. 227-34. }\end{array}$ & 1997 & Helmenstein & Christian & IHS & 0.26 & 3 & 0.5 & 0.065 & \\
\hline $\begin{array}{l}\text { Migrants' Savings, Purchasing Power } \\
\text { Parity, and the Optimal Duration of } \\
\text { Migration }\end{array}$ & $\begin{array}{l}\text { Stark,-O.; } \\
\text { Helmenstein,-C.; } \\
\text { Yegorov,-Y. }\end{array}$ & \begin{tabular}{|l|} 
International-Tax-and- \\
Public-Finance; 4(3), July \\
1997, pp. 307-24.
\end{tabular} & 1997 & Helmenstein & Christian & IHS & 0 & 3 & 1 & 0 & \\
\hline $\begin{array}{l}\text { Inflation and Interest Rate } \\
\text { Differentials between Germany and } \\
\text { Its EMS Partners }\end{array}$ & $\begin{array}{l}\text { Helmenstein,-- } \\
\text { Christian; Runstler,- } \\
\text { Gerhard }\end{array}$ & $\begin{array}{l}\text { Zeitschrift-fur-Wirtschafts-- } \\
\text { und-Sozialwissenschaften; } \\
116(4), 1996 \text {, pp. 593-613. }\end{array}$ & 1996 & Helmenstein & Christian & IHS & 0 & 2 & 1 & 0 & \\
\hline $\begin{array}{l}\text { Neural Networks in the Capital } \\
\text { Markets: An Application to Index } \\
\text { Forecasting }\end{array}$ & $\begin{array}{l}\text { Haefke,-Christian; } \\
\text { Helmenstein,-- } \\
\text { Christian }\end{array}$ & $\begin{array}{l}\text { Computational-Economics; } \\
\text { 9(1), February 1996, } \\
\text { pp. } 37-50 \text {. }\end{array}$ & 1996 & Helmenstein & Christian & IHS & 0 & 2 & 1 & 0 & \\
\hline Kleine Wahrungsunion in Europa. & $\begin{array}{l}\text { Helmenstein,-- } \\
\text { Christian; Url,-- } \\
\text { Thomas }\end{array}$ & $\begin{array}{l}\text { Konjunkturpolitik; 39(4), } \\
\text { 1993, pp. 251-63. }\end{array}$ & 1993 & Helmenstein & Christian & IHS & 0 & 2 & 1 & 0 & $\begin{array}{l}\text { C. Helmenstein: } \\
1.023333333\end{array}$ \\
\hline $\begin{array}{l}\text { Prediction Risk and the Forecasting } \\
\text { of Stock Market Indexes }\end{array}$ & $\begin{array}{l}\text { Haefke,-Christian; } \\
\text { Helmenstein,- } \\
\text { Christian }\end{array}$ & $\begin{array}{l}\text { Operations Research and } \\
\text { Decision; 3-4, 1996, } \\
\text { pp. 131-149. }\end{array}$ & 1996 & Helmenstein & Christian & IHS & 0.72 & 2 & 1 & 0.48 & \\
\hline
\end{tabular}




\begin{tabular}{|c|c|c|c|c|c|c|c|c|c|c|c|}
\hline Titel & Author(s) & Journal & Year & & & & $\begin{array}{l}\text { Impact } \\
\text { Factor }\end{array}$ & Authors & $\begin{array}{l}\text { Page- } \\
\text { corr. }\end{array}$ & Impact & personal Impact \\
\hline $\begin{array}{l}\text { Forecasting Austrian IPOs: An } \\
\text { Application of Linear and Neural } \\
\text { Network ... }\end{array}$ & $\begin{array}{l}\text { Haefke,-Christan; } \\
\text { Helmenstein,- } \\
\text { Christian }\end{array}$ & $\begin{array}{l}\text { Journal of Forecasting, } \\
\text { vol. 15, April 1996, } \\
\text { pp. 237-251. }\end{array}$ & 1996 & Helmenstein & Christian & IHS & 0.62 & 2 & 1 & 0.413333333 & \\
\hline $\begin{array}{l}\text { Anbieterkonzentration auf dem Markt } \\
\text { für Jahresabschlußprüfungen }\end{array}$ & $\begin{array}{l}\text { Helmenstein,- } \\
\text { Christian }\end{array}$ & $\begin{array}{l}\text { DBW - Die } \\
\text { Betriebswirtschaft, vol. } \\
\text { 56(1), January/February } \\
\text { 1996, pp. 41-48. }\end{array}$ & 1996 & Helmenstein & Christian & IHS & 0 & 1 & 0.5 & 0 & \\
\hline $\begin{array}{l}\text { Regional Per Capita Income } \\
\text { Convergence in Austria }\end{array}$ & \begin{tabular}{|l|} 
Hofer,-Helmut; \\
Worgotter,-Andreas
\end{tabular} & $\begin{array}{l}\text { Regional-Studies; 31(1), } \\
\text { February 1997, pp. 1-12. }\end{array}$ & 1997 & Hofer & Helmut & IHS & 0.71 & 2 & 1 & 0.473333333 & $\begin{array}{l}\text { H. Hofer: } \\
0.946666667 \\
\end{array}$ \\
\hline $\begin{array}{l}\text { Housing Investment Dynamics, } \\
\text { Period of Production, and Adjustment } \\
\text { Cost }\end{array}$ & \begin{tabular}{|l|} 
Lee, Gabriel \\
\end{tabular} & $\begin{array}{l}\text { Journal of Housing } \\
\text { Economics, vol. 8, no.1, } \\
\text { 1999, pp. 1-25 } \\
\end{array}$ & 1999 & Lee & Gabriel & $\mathbb{H S}$ & 0 & 1 & 1 & 0 & \\
\hline $\begin{array}{l}\text { Housing Cycles and the Period of } \\
\text { Production }\end{array}$ & Lee, Gabriel & $\begin{array}{l}\text { Applied Economics, vol. } \\
31,1999, \text { pp. 1219-1230. }\end{array}$ & 1999 & Lee & Gabriel & IHS & 0.28 & 1 & 1 & 0.28 & G. Lee: 0.28 \\
\hline Recall in Extensive Form Games & Ritzberger, -Klaus & \begin{tabular}{|l|} 
International-Journal-of- \\
Game-Theory; $28(1)$, \\
February 1999, pp. 69-87
\end{tabular} & 1999 & Ritzberger & Klaus & IHS & 0.31 & 1 & 1 & 0.31 & \\
\hline $\begin{array}{l}\text { Imperfectly Observable Commitments } \\
\text { in n-Player Games }\end{array}$ & \begin{tabular}{|l|} 
Guth,-W.; \\
Kirchsteiger,-G.; \\
Ritzberger,-K. \\
\end{tabular} & $\begin{array}{l}\text { Games-and-Economic- } \\
\text { Behavior; 23(1), April } \\
\text { 1998, pp. 54-74. } \\
\end{array}$ & 1998 & Ritzberger & Klaus & IHS & 0.6 & 3 & 1 & 0.3 & \\
\hline $\begin{array}{l}\text { On Durable Goods Monopolies and } \\
\text { the Coase-Conjecture }\end{array}$ & $\begin{array}{l}\text { Guth,-Werner; } \\
\text { Ritzberger,-Klaus }\end{array}$ & $\begin{array}{l}\text { Review -of-Economic- } \\
\text { Design; 3(3), 1998, } \\
\text { pp. 215-236. } \\
\end{array}$ & 1998 & Ritzberger & Klaus & IHS & 0 & 2 & 1 & 0 & \\
\hline $\begin{array}{l}\text { On Games under Expected Utility } \\
\text { w ith Rank Dependent Probabilities }\end{array}$ & Ritzberger,-Klaus & $\begin{array}{l}\text { Theory-and-Decision; } \\
\text { 40(1), January 1996, } \\
\text { pp. 1-27. }\end{array}$ & 1996 & Ritzberger & Klaus & IHS & 0.56 & 1 & 1 & 0.56 & \\
\hline $\begin{array}{l}\text { Optimal Provision against the Risks of } \\
\text { Old Age }\end{array}$ & \begin{tabular}{|l|} 
Richter,-Wolfram-F.; \\
Ritzberger,-Klaus \\
\end{tabular} & $\begin{array}{l}\text { FinanzArchiv; 52(3), 1995, } \\
\text { pp. 339-56. }\end{array}$ & 1995 & Ritzberger & Klaus & IHS & 0 & 2 & 1 & 0 & \\
\hline $\begin{array}{l}\text { On Durable Goods Monopolies: An } \\
\text { Experimental Study ... }\end{array}$ & $\begin{array}{l}\text { Guth,-W.; Ockenfels,-- } \\
\text { P.; Ritzberger,-K. }\end{array}$ & \begin{tabular}{|l|} 
Journal-of-Economic- \\
Psychology; 16(2), July \\
1995, pp. 247-74. \\
\end{tabular} & 1995 & Ritzberger & Klaus & IHS & 0.24 & 3 & 1 & 0.12 & \\
\hline $\begin{array}{l}\text { Evolutionary Selection in Normal- } \\
\text { Form Games }\end{array}$ & $\begin{array}{l}\text { Ritzberger,-Klaus; } \\
\text { Weibull,-Jorgen-W. }\end{array}$ & $\begin{array}{l}\text { Econometrica; 63(6), } \\
\text { November 1995, } \\
\text { pp. 1371-99. } \\
\end{array}$ & 1995 & Ritzberger & Klaus & IHS & 2.22 & 2 & 1 & 1.48 & \\
\hline $\begin{array}{l}\text { The Theory of Normal Form Games } \\
\text { form the Differentiable Viewpoint }\end{array}$ & Ritzberger,-Klaus & $\begin{array}{l}\text { International-Journal-of- } \\
\text { Game-Theory; } 23(3) \\
\text { 1994, pp. 207-36. }\end{array}$ & 1994 & Ritzberger & Klaus & IHS & 0.31 & 1 & 1 & 0.31 & \\
\hline $\begin{array}{l}\text { Family Allowances as Welfare } \\
\text { Improvements }\end{array}$ & $\begin{array}{l}\text { Felderer,-Bernhard; } \\
\text { Ritzberger,-Klaus }\end{array}$ & \begin{tabular}{|l|} 
Journal-of-Economics- \\
$($ (ZfN); 61(1), 1995, pp. 11- \\
33.
\end{tabular} & 1995 & Ritzberger & Klaus & IHS & 0.18 & 2 & 1 & 0.12 & \\
\hline $\begin{array}{l}\text { Dynamische Modelle Evolutionarer } \\
\text { Selektion }\end{array}$ & Ritzberger,-Klaus & $\begin{array}{l}\text { Ifo-Studien; 39(3-4), 1993, } \\
\text { pp. 221-38. }\end{array}$ & 1993 & Ritzberger & Klaus & IHS & 0 & 1 & 1 & 0 & \\
\hline $\begin{array}{l}\text { Optimal Biproportional Updating of } \\
\text { Input-Output Matrices ... }\end{array}$ & Ritzberger,-Klaus & $\begin{array}{l}\text { Economic-Notes; 0(1), } \\
1990, \text { pp. 82-101. }\end{array}$ & 1990 & Ritzberger & Klaus & IHS & 0 & 1 & 1 & 0 & K. Ritzberger: 3.2 \\
\hline
\end{tabular}

Average: 1.11025641 